\title{
The Near-Earth Plasma Environment
}

\author{
Robert F. Pfaff Jr.
}

Received: 1 March 2012 / Accepted: 3 March 2012 / Published online: 20 June 2012

(C) US Government 2012

\begin{abstract}
An overview of the plasma environment near the earth is provided. We describe how the near-earth plasma is formed, including photo-ionization from solar photons and impact ionization at high latitudes from energetic particles. We review the fundamental characteristics of the earth's plasma environment, with emphasis on the ionosphere and its interactions with the extended neutral atmosphere. Important processes that control ionospheric physics at low, middle, and high latitudes are discussed. The general dynamics and morphology of the ionized gas at mid- and low-latitudes are described including electrodynamic contributions from wind-driven dynamos, tides, and planetary-scale waves. The unique properties of the near-earth plasma and its associated currents at high latitudes are shown to depend on precipitating auroral charged particles and strong electric fields which map earthward from the magnetosphere. The upper atmosphere is shown to have profound effects on the transfer of energy and momentum between the high-latitude plasma and the neutral constituents. The article concludes with a discussion of how the near-earth plasma responds to magnetic storms associated with solar disturbances.
\end{abstract}

Keywords Ionosphere $\cdot$ Upper atmosphere $\cdot$ Electric fields

\section{Introduction}

In this article we discuss the near-earth plasma environment, for which the ionosphere is the natural focus. Because this ionized gas co-exists with the neutral atmospheric gas to which it is coupled, the ionized/neutral gas system is more properly considered a partially ionized gas rather than a medium consisting of two distinct, independent fluids. We discuss the origins of the ionized component and describe its general morphology and dynamics, both in the framework of the earth's magnetic field as well as with respect to its coupling

Contributed to the ISSI Monograph entitled: "Coupling of the Earth's Atmosphere and Its Plasma Environment".

R.F. Pfaff Jr. ( $\varangle)$

NASA/Goddard Space Flight Center, Greenbelt, MD 20771, USA

e-mail: Robert.F.Pfaff@nasa.gov 


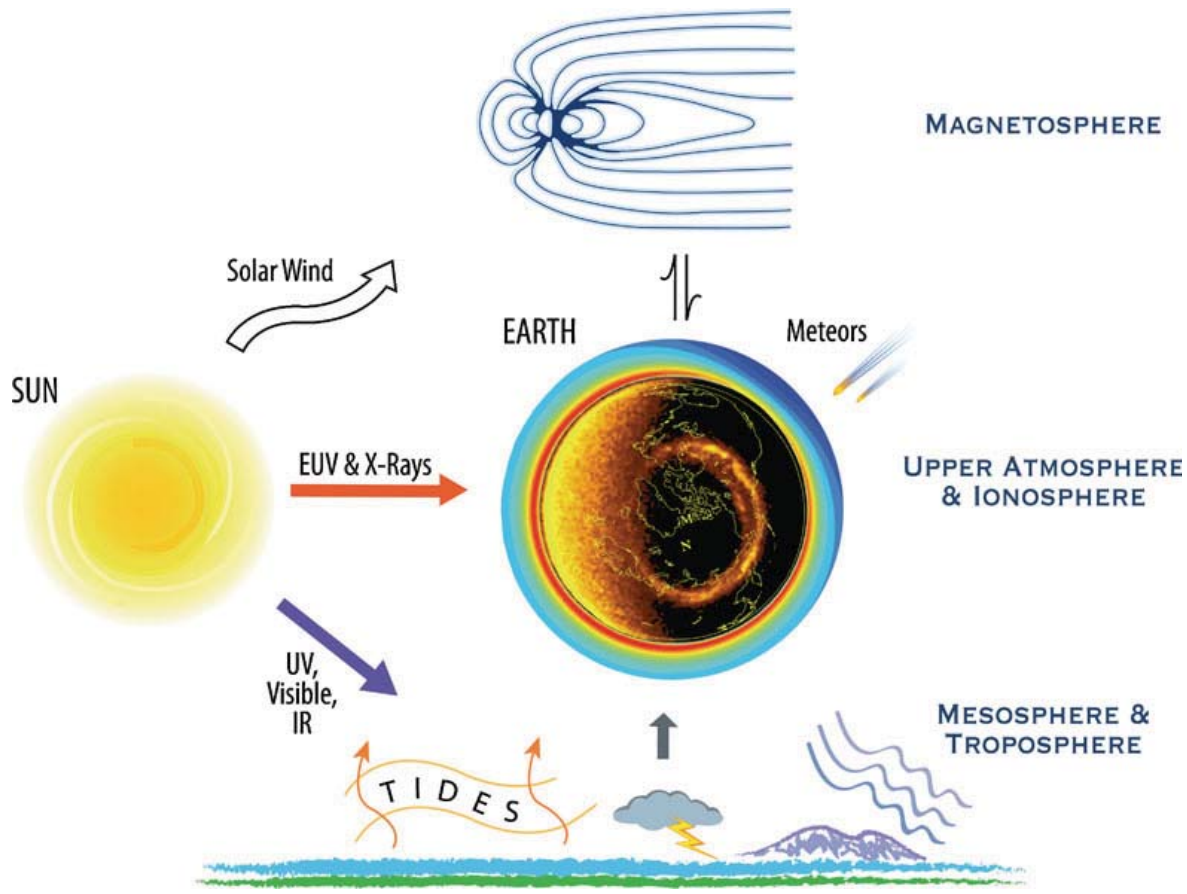

Fig. 1 The various paths of solar energy to the earth's upper atmosphere and ionosphere as well as its coupling to the magnetosphere above and the mesosphere and troposphere below

to the neutral atmosphere and its motions. Because of the emphasis of this monograph on atmosphere-plasma coupling, and in order to keep the article manageable, we do not include a detailed discussion of the earth's magnetosphere plasma, except in its role of exchanging energy and momentum with the partially ionized atmosphere at its base.

We discuss two main mechanisms by which ambient or "thermal" plasma is created in the near-earth environment, each of which depends on an interaction of an external energy source with the earth's extended atmosphere: (1) photo-ionization of the neutral gas via impinging extreme ultraviolet (EUV) solar radiation and (2) impact ionization of the neutral gas via energetic particle precipitation. A second form of impact ionization, due to meteor ablation, is also briefly discussed. Whereas photo-ionization is responsible for the creation of the primary part of earth's ionosphere, particularly at mid- and low-latitudes, at high latitudes impact ionization by precipitating, energetic particles creates thermal plasma with different properties and features. Although their ionizing mechanisms are driven by different energy sources, the resulting plasma in each case is a consequence of energy or momentum impact with the upper atmosphere itself. Indeed, as we shall see, it is precisely because the earth has an extended atmosphere that it also has an ionosphere. In fact, any planet with a sensible, gravitationally-bound atmosphere and an ionizing source of radiation of sufficient energy and intensity, will consequently form an ionosphere. For planets which also have an intrinsic magnetic field and a substantial rotation rate, such as the earth, the resulting plasma environment may be expected to be particularly dynamic and rich in its complexity and physical processes.

In order to illustrate the context of the near-earth plasma within the larger sun-earth system, we depict the main pathways by which energy and momentum are deposited and exchanged within the earth's upper atmosphere in Fig. 1. Here, one path shows how the sun directly influences the upper atmosphere via EUV and X-ray radiation, both via heating of the neutral atmosphere, which results in its expansion, and also via photo-ionization of a 
small fraction of this same neutral gas which thereby re-generates the ionosphere throughout the day. A second path shows how solar wind processes, including plasma flow with embedded interplanetary magnetic fields, interact with the magnetosphere to drive largescale electric fields, currents, and energetic particles that subsequently interact with the underlying upper atmosphere/ionosphere. Such solar wind/magnetospheric input impacts the near-earth plasma primarily at high latitudes, although it may dramatically influence all latitudes during periods of magnetic storms. Although the globally averaged energy input by the solar wind/magnetosphere is not as high as the total solar photon irradiance, its impact in the upper atmosphere is nonetheless quite significant, and locally it can be the dominant energy source. The diagram in Fig. 1 also includes a sketch showing that the upper atmosphere is also influenced by energy and momentum sources from below, namely from electrical discharges in the troposphere (lightning) and mesosphere (e.g., sprites), as well as via upward-propagating tides, planetary waves, and gravity waves. Such sources have profound effects on the characteristics of the mid- and low-latitude ionosphere in particular. The energy source that heats and sets in motion the lower and middle atmosphere is, again, solar radiation, but in this case, its ultraviolet, visible, and infrared wavelengths.

An outline of this article is as follows: We begin with a description of how the earth's ionosphere is formed via photo-ionization, and its daily variation. We then discuss how these ionized particles are influenced by the earth's magnetic field and how they couple to the neutral atmospheric gas via collisions, as represented by the mobility tensor for electrons and ions. The dynamics and morphology of the ionosphere at low and mid-latitudes are then discussed, followed by a separate section on the earth's high latitude ionosphere including the aurora and the exchange of energy and momentum with the magnetosphere. Finally, we discuss the effects of magnetic storms on the ionosphere and conclude with a brief summary.

There are several excellent references that describe the earth's ionosphere and upper atmosphere. These include: Hargreaves (1992), Kelley (2009), Prölss (2004), Rishbeth and Garriott (1969), and Schunk and Nagy (2009), among others.

\section{Creation of the Earth's Ionosphere via Photo-ionization and Its Daily Variation}

In this section, we describe how the ionosphere is created by photo-ionization associated with solar EUV and X-ray radiation that impacts the earth's tenuous upper atmosphere. We also describe how some positive ions thus produced may transfer their charge to different neutral species and how others subsequently recombine with free electrons to form neutral gas particles. We conclude with a general description of how the mid- and low-latitude ionospheric density and temperature vary with local time and altitude.

\subsection{The Earth's Extended Neutral Atmosphere}

In the Introduction, we discussed how the external sources of energy and momentum impinge on the earth's atmosphere to create thermal plasma via two main mechanisms: solar radiation induced photo-ionization and impact ionization from energetic particles and meteors. Before discussing these ionization processes, we first review some of the fundamental features of the Earth's upper neutral atmosphere in order to illustrate how they influence the resulting plasma parameters formed by these ionizing mechanisms.

Whereas the earth's atmosphere near the surface consists primarily of nitrogen molecules $\left(\mathrm{N}_{2}\right)$ and oxygen molecules $\left(\mathrm{O}_{2}\right)$, the atmospheric constituents change markedly at higher 


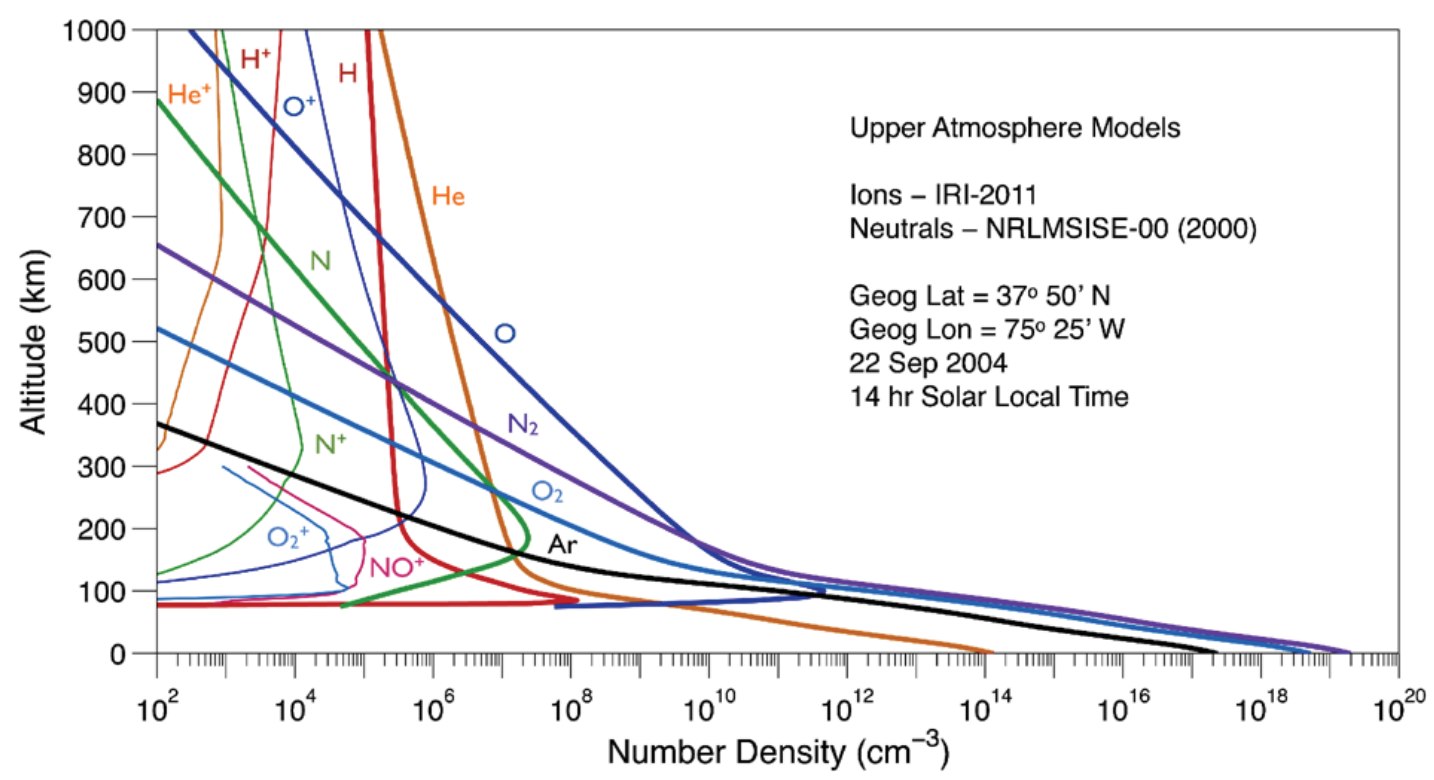

Fig. 2 Model distributions of neutral and ionic constituents as a function of altitude for a mid-latitude location (Wallops Island, Va., USA) at $14 \mathrm{hr}$ Solar Local Time. Ion distributions below $80 \mathrm{~km}$ are not provided by the model

altitudes. At about $100 \mathrm{~km}$ and above, the atmosphere ceases to be homogeneous and converts to free-molecular flow with a large mean-free path of the particles. Additionally, the atmospheric constituents above about $100 \mathrm{~km}$ directly absorb solar EUV radiation. Because of this absorption and the fact that these gases are very ineffective infrared radiators, the neutral temperatures increase significantly with altitude above $100 \mathrm{~km}$. Consequently, this region of the upper atmosphere is often referred to as the thermosphere.

The main process by which the upper atmosphere absorbs EUV energy from the sun is photo-dissociation. Diatomic oxygen is the primary molecule in the upper atmosphere that dissociates. This occurs when it is struck by photons with wavelengths of $242 \mathrm{~nm}$ or less, as expressed by the reaction:

$$
\mathrm{O}_{2}+h v(\lambda<242 \mathrm{~nm}) \rightarrow \mathrm{O}+\mathrm{O}+\text { energy }
$$

The creation of atomic oxygen is important because it is readily photo-ionized, as discussed later.

The various species of atoms and molecules that make up the atmosphere are shown in Fig. 2, for mid-latitude, daytime conditions with moderate solar activity, as computed from the NRLMSISE-2000 model (Picone et al. 2002). The concentrations per unit volume of the atmospheric constituents and their variation with altitude affect the ionosphere in two ways: First, they determine the amount of neutral gas available for photo-ionization at different altitudes, and second, they determine to what depth the solar EUV and X-ray radiation might penetrate, as discussed in the next section. For example, a consequence of a decreased neutral atmospheric density is that photons can penetrate to lower altitudes.

Both the neutral and plasma densities in the earth's upper atmosphere vary considerably because of EUV solar radiation variations associated with solar activity. Unlike the main portion of the sun's emission spectrum, for example where the visible light is emitted, the EUV portion of the spectrum originates at the base of the highly dynamic corona, where it undergoes large variations in accordance with solar storms and solar activity. In fact, the EUV radiation typically changes by factors of 2-3 (sometimes larger) within the solar cycle. 
Fig. 3 Representative plasma density profiles for day and night at solar minimum and maximum conditions at a mid-latitude location (Hargreaves 1992). The colored arrows show the approximate penetration depths of the X-ray, EUV, and UV radiation from the sun

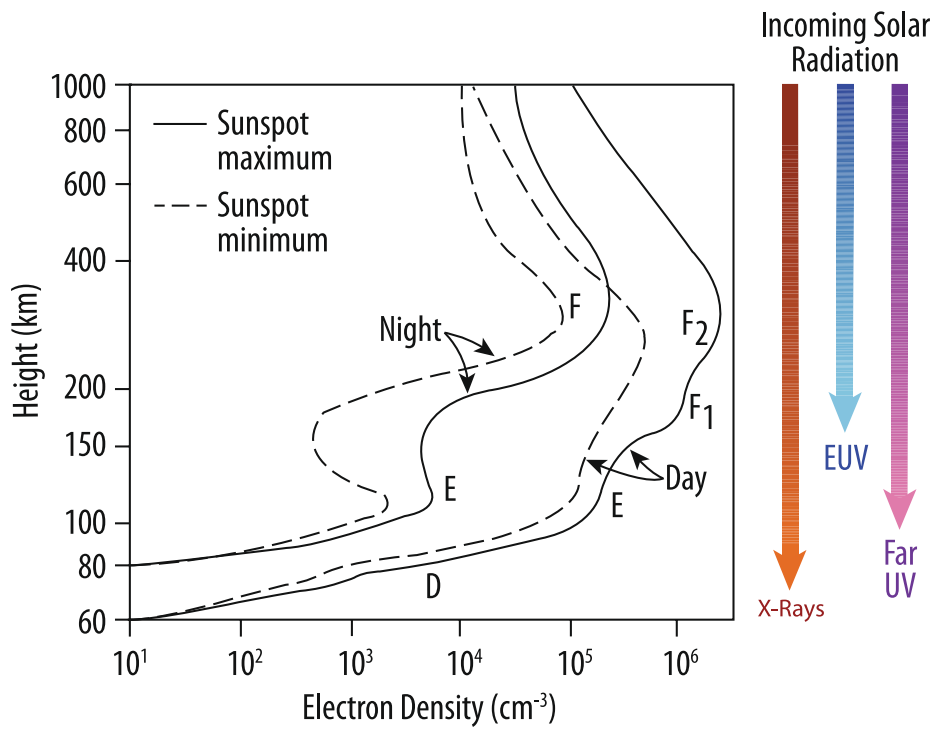

The EUV radiation is doubly important for determining the characteristics of the ionosphere as it: (1) is absorbed by the upper atmosphere and thereby heats the atmosphere, causing it expand, as discussed above, and (2) photo-ionizes a small fraction of the gas, creating the ionosphere, as discussed below.

\subsection{Photo-Ionization, Charge Exchange, and Recombination}

The main mechanism that creates the ionosphere is the photo-ionization of a tiny fraction of the earth's extended, tenuous neutral upper atmosphere during the daytime. During the night, there is a very small amount of radiation input resulting from the high altitude scattering of the solar radiation into the shadow of the planet and other minor sources. Incoming solar EUV and X-ray radiation penetrate the upper atmosphere with sufficient energy to detach an electron from a neutral atom to create an ion and electron pair, as depicted here for oxygen, the main atmospheric gas ionized in this manner:

$$
\mathrm{O}+h v \rightarrow \mathrm{O}^{+}+\mathrm{e}^{-}
$$

This radiation penetrates to different altitudes based on wavelength. Enhanced plasma layers are formed where the intensity of the incoming radiation, which falls off with decreasing altitude, and the amount of a particular gas constituent available to be ionized, which falls off with increasing altitude, are optimal, as discussed by Chapman (1931). For the earth, two main layers of ionization are created in the daytime ionosphere due to the photo-ionization of oxygen atoms: one formed by soft X-rays around $110 \mathrm{~km}$, denoted as the E region and one formed by EUV radiation near $150 \mathrm{~km}$ denoted as the $\mathrm{F} 1$ region.

The resulting ionization plotted as a function of altitude is shown in the left hand side of Fig. 3, for a mid-latitude location (Hargreaves 1992). Note the strong dependence of the resulting plasma density on solar activity, which directly affects the strength of available EUV and X-ray radiation as discussed above, as well as how the profiles differ between day and night, particularly at the lower altitudes (discussed below). The incoming ionizing radiation is represented schematically on the right hand side of the figure. The actual penetration altitudes at a given latitude vary during the day with the solar zenith angle. For large solar zenith angles, grazing incidence corrections must be taken into account. (See discussion in Rishbeth and Garriott 1969 and references therein.) 
The number density distributions of the different ion species which constitute the ionosphere are shown in Fig. 2, taken from the International Reference Ionosphere (IRI) empirical model (Bilitza and Reinisch 2008; Bilitza et al. 2011), for the same mid-latitude, daytime conditions used for the neutral density profiles shown in this figure. The curves show how the various ion constituents change with altitude. Indeed, the distribution of ions within the ionosphere is controlled to a large extent by the distributions of the neutral gas constituents. When comparing the ionized density profile with that of the neutral density profile shown in Fig. 2, notice that, for altitudes below about $300 \mathrm{~km}$, the plasma number densities are $10^{4}$ to $10^{6}$ times smaller than those of the neutral parameters.

It is important to emphasize that photo-ionization is sometimes only the first step in a chain of processes by which the ambient ion gases are ultimately formed. For example, oxygen atoms are readily photo-ionized while atmospheric molecules, such as $\mathrm{O}_{2}$ and NO, do not photo-ionize nearly as efficiently. However, charge exchange readily occurs between the oxygen ions and the ambient nitrogen and oxygen molecules $\left(\mathrm{N}_{2}\right.$ and $\left.\mathrm{O}_{2}\right)$, creating $\mathrm{NO}^{+}$ and $\mathrm{O}_{2}^{+}$:

$$
\begin{aligned}
& \mathrm{O}^{+}+\mathrm{N}_{2} \rightarrow \mathrm{NO}^{+}+\mathrm{N} \\
& \mathrm{O}^{+}+\mathrm{O}_{2} \rightarrow \mathrm{O}_{2}^{+}+\mathrm{O}
\end{aligned}
$$

At the lower altitudes, the resulting molecular ions are the dominant ones, even though the initial ionization is produced by photo-ionization of oxygen atoms. This is an important aspect of the near-earth plasma because the molecular ionization is lost quickly through recombination when their ionization source is cut off.

As soon as the positive ions and electrons are freed, they are subject to chemical recombination processes as well as charge exchange and transport processes. The most common of these processes is dissociative recombination, which is important for the E-region molecular ions:

$$
\begin{aligned}
& \mathrm{O}_{2}^{+}+\mathrm{e} \rightarrow \mathrm{O}+\mathrm{O} \\
& \mathrm{NO}^{+}+\mathrm{e} \rightarrow \mathrm{N}+\mathrm{O}
\end{aligned}
$$

This is a rather fast process in which newly formed $\mathrm{O}_{2}^{+}$and $\mathrm{NO}^{+}$typically recombine in seconds. In fact, the loss rate for the molecular species, $L$, is given by: $L=C_{r} N_{i} N_{e}$, where $C_{r}$ is the recombination coefficient, $N_{i}$ is the ion density, and $N_{e}$ is the electron density. Since the loss rate depends on the ion density, if the molecular species is the dominant ion, the loss rate is very rapid. On the other hand, $\mathrm{O}^{+}$recombines via a radiative process:

$$
\mathrm{O}^{+}+\mathrm{e} \rightarrow \mathrm{O}+h v
$$

which is very slow. In fact, $\mathrm{O}^{+}$is far more likely to be lost via charge exchange or other processes rather than through recombination. Since the molecular ions dominate the lower altitudes (i.e., less than $200 \mathrm{~km}$ ), when the direct illumination of the sun vanishes at dusk, the lower ionosphere, which rotates with the planet, becomes largely depleted of any significant plasma until local sunrise at which time the main photo-ionization process starts over again. In this fashion, we see that at the lower altitudes, the ionosphere is continuously being reborn every day.

The physical processes and chemistry associated with the ion and neutral gas constituents, with particular emphasis on ionization and recombination, constitutes the general domain of aeronomy, the science of the upper atmosphere. A detailed discussion of these processes may be found in Rishbeth and Garriott (1969), Banks and Kockarts (1973), Prölss (2004), and numerous other texts. 


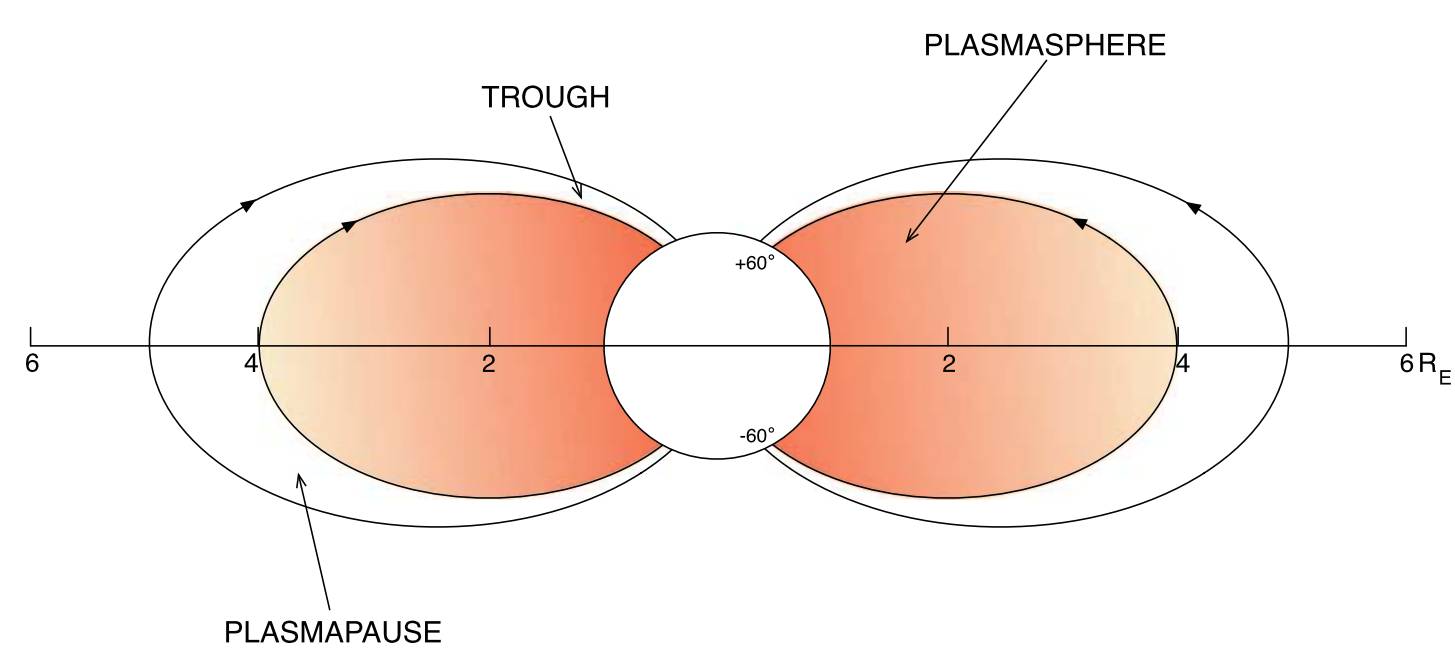

Fig. 4 The plasmasphere, an extension of the ionosphere at mid and low latitudes. The plasmasphere is confined to closed magnetic field lines and extends to approximately 4 earth radii at the equator

\subsection{The F2 Peak, Protons, and the Plasmasphere}

Returning to Fig. 3, we see that the majority of the ionospheric plasma peaks at altitudes above the F1 layer, known as the F2 peak. Although this layer dominates the ionosphere, it is not formed primarily by direct photo-ionization occurring at this altitude, but instead is the result of other, subsequent processes, including for example, the upwards diffusion of $\mathrm{O}^{+}$ions to higher altitudes where they do not encounter significant numbers of molecules with which to charge exchange. In addition, neutral wind drag-driven ion flows are believed to be another significant process that contributes to the F2 ionization peak properties at midand low-latitudes, as discussed by Rishbeth (1967). Finally, upward plasma transport due to electric fields, particularly during the daytime, also produces changes in the F2 peak, as discussed in Sect. 4.

Note further in Fig. 2 that the lighter hydrogen ion, $\mathrm{H}^{+}$, does not become dominant until much higher altitudes. Within the "topside" ionosphere (i.e., above the F2 peak), charge exchange between oxygen ions and hydrogen atoms is common, accounting for all of the protons of ionospheric origin at mid and low latitudes:

$$
\mathrm{O}^{+}+\mathrm{H} \rightarrow \mathrm{H}^{+}+\mathrm{O}
$$

For moderate solar activity, at altitudes above about $1000 \mathrm{~km}$, the $\mathrm{H}^{+}$ion dominates, and this region is often referred to as the protonosphere. The amount of $\mathrm{H}^{+}$present varies considerably between day and night and is also highly dependent on solar activity. For example, Heelis et al. (2009a, 2009b) showed that during extreme solar minimum conditions, the crossover altitudes of $\mathrm{O}^{+}$and $\mathrm{H}^{+}$ions could be as low as $450 \mathrm{~km}$ at night. $\mathrm{He}^{+}$also increases in importance with altitude, although, except possibly during geomagnetic storms, it is typically minor compared to $\mathrm{H}^{+}$.

The large concentrations of light ions extend to altitudes of about 4 earth radii at the equator, as shown in Fig. 4. This high-altitude region is called the plasmasphere and is basically a torus that surrounds the mid- and low-latitude portions of the earth and is considered an upward extension of the ionosphere at magnetic latitudes below about 60 degrees. The plasmaspheric plasma is trapped on closed magnetic field lines that rotate with the Earth and thus the plasmasphere co-rotates with them. (The earth's magnetic field is discussed in the next major section.) The outer plasmasphere is composed mostly of hydrogen ions with 
singly ionized helium accounting for approximately 10-20\% of the plasma (e.g., Craven et al. 1997) and has temperatures of roughly $1 \mathrm{eV}$ (or 11,600 K). The plasmasphere terminates at a sharp decrease in density known as the plasmapause. At lower altitudes, it is sometimes associated with a sharp decrease in the topside ionosphere with increasing latitude that is referred to as the trough. The trough occurs near 60 degrees magnetic latitude, and is also used to define the equatorward edge of the high-latitude ionosphere (see review by Rodger 2008).

\subsection{Temperature of the Near-Earth Plasma}

In addition to its number density, the near-earth plasma is also characterized by its temperature. For the photo-ionization process described above, when an atom absorbs a photon and emits an electron, the resulting photoelectron typically has an energy of several $\mathrm{eV}$. This energy then dissipates via inelastic collisions with the neutral gas. The electron has a higher velocity than that of the ion at the same temperature due to its much smaller mass and the conservation of momentum. The resulting temperature distribution of each species depends on their collisions with other neutral and ionized particles and thus varies with altitude and geographic position.

Typical average values of electron and ion temperatures vary between $350-3000 \mathrm{~K}$, or $0.03-0.3 \mathrm{eV}$, as shown in Fig. 5 for conditions at the magnetic equator at dawn, noon, sunset, and midnight as computed from the IRI model. In the lower ionosphere $(<120 \mathrm{~km})$, the average values of the electron, ion, and neutral gas temperatures $\left(T_{e}, T_{i}\right.$ and $\left.T_{n}\right)$ are similar due to the increased number of collisions with the over-abundant neutrals. The values of $T_{e}, T_{i}$, and $T_{n}$ then diverge with increasing altitude. The electron temperature is highest in the early morning. The neutrals, rotating into sunlight, have yet to warm and the neutral atmosphere is still contracted from the colder, nightside temperature. Hence, the electronneutral collisions are less frequent. During the day, the neutral atmosphere expands, and the electron-neutral collision frequency thus increases at the higher altitudes, reducing the

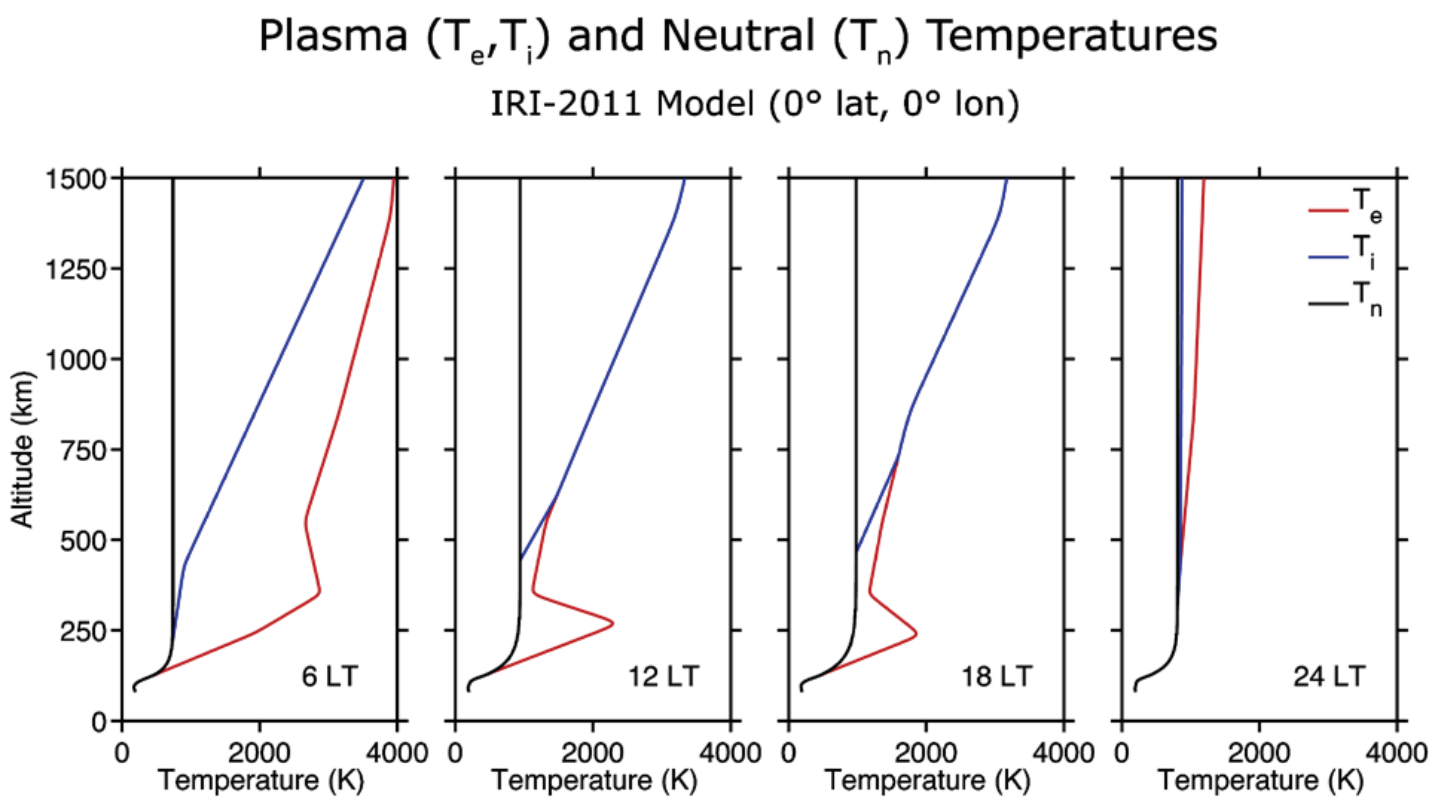

Fig. 5 IRI model calculations showing the plasma and neutral temperatures at the magnetic equator at four different local times 
electron temperature. The electron temperature further decreases during the day as it equilibrates with the ion temperature due to electron-ion collisions. The temperatures are largest at the higher altitudes where the neutral and plasma densities are low. The enhanced electron temperature near $300 \mathrm{~km}$ during the day results from photoelectrons produced within the upper altitude portion of the layer where the neutral atmosphere is less dense. At night, there are no newly-formed photoelectrons and the electron, ion and neutral temperatures quickly converge to similar values via collision processes. See Schunk and Nagy (2009) for a detailed discussion of the neutral and plasma temperature variations.

At higher latitudes, precipitating particles, magnetospheric electric fields, and electric currents also play significant roles in heating the ionosphere. Frictional heating (Joule heating) between the ions and neutrals in particular contributes significantly to ion and neutral heating at high latitudes, and plasma instabilities at low altitudes (e.g., two-stream instabilities, particularly in the auroral zone) may produce elevated electron temperatures locally.

\subsection{Metallic Ions}

Another source of ions to the earth's near-earth plasma results from the influx of meteoroids. The earth is laden by $\sim 10^{8} \mathrm{~kg} /$ year of meteoritic material (e.g., Ceplecha 1992), depositing dust, grains, and atoms released by ablation. As these particles settle due to gravity, metallic atoms may undergo charge exchange to form metallic ions such as $\mathrm{Fe}^{+}, \mathrm{Mg}^{+}$, and $\mathrm{Na}^{+}$, and photo-ionization may also occur (Kopp 1997).

The main source of metallic ions in the ionosphere, however, is impact ionization by meteors striking the atmosphere. This mechanism directly produces ionized gases at the meteor head, which occurs when the kinetic energy of the incoming meteoroid is sufficient to remove electrons from the neutrals (e.g., Vondrak et al. 2008). Whether a meteoroid will undergo impact ionization depends on its mass, velocity, and angle of attack with respect to the atmosphere. Such ionization is observed through radar echoes of meteor trails, as they interact with the atmospheric gas primarily between $80-110 \mathrm{~km}$, forming cylinders of ionizing gas often orders of magnitude denser than that of the surrounding plasma (e.g., Dyrud et al. 2001; Close et al. 2002; Janches et al. 2009). An example of such radar "head echoes" associated with the ionized gas at the head of an ablating meteor is shown in Fig. 6 (Chapin and Kudeki 1994). Notice in particular how the head echo extends for over $15 \mathrm{~km}$ in altitude. The head echoes are followed by a radar trail of plasma irregularities with organized scatter that, in this case, lasted for several minutes as they were formed near the magnetic equator. Such head echoes are commonly detected by ionospheric radars at all latitudes and illustrate the prevalence of meteor-produced ionization at low altitudes (e.g., Mathews et al. 2008).

The glowing gas associated with ablation of larger meteoroids produces the familiar "shooting stars" that are observed in the night sky at all latitudes and which are most prevalent in the pre-dawn hours when the earth's proper motion directly sweeps through the interplanetary medium. For such emissions to be visible to the unaided eye, the mass of the meteoroid is generally on the order of milligrams, whereas "radar" meteors have considerably smaller masses, on the order of 1-100 micrograms, and their ablation optical signature is generally not visible to the unaided eye. Ablation of these smaller meteoroids are much more frequent and are believed to be a more significant source of metallic ions, at least compared to that produced by the larger meteoroids (D. Janches, personal communication, 2012).

Metallic ions in the earth's ionosphere have recombination lifetimes for neutralization that are very long. Thus, the long lasting metallic ions generally undergo subsequent motions after they are created. For example, as the metallic ions settle in the lower portion of 


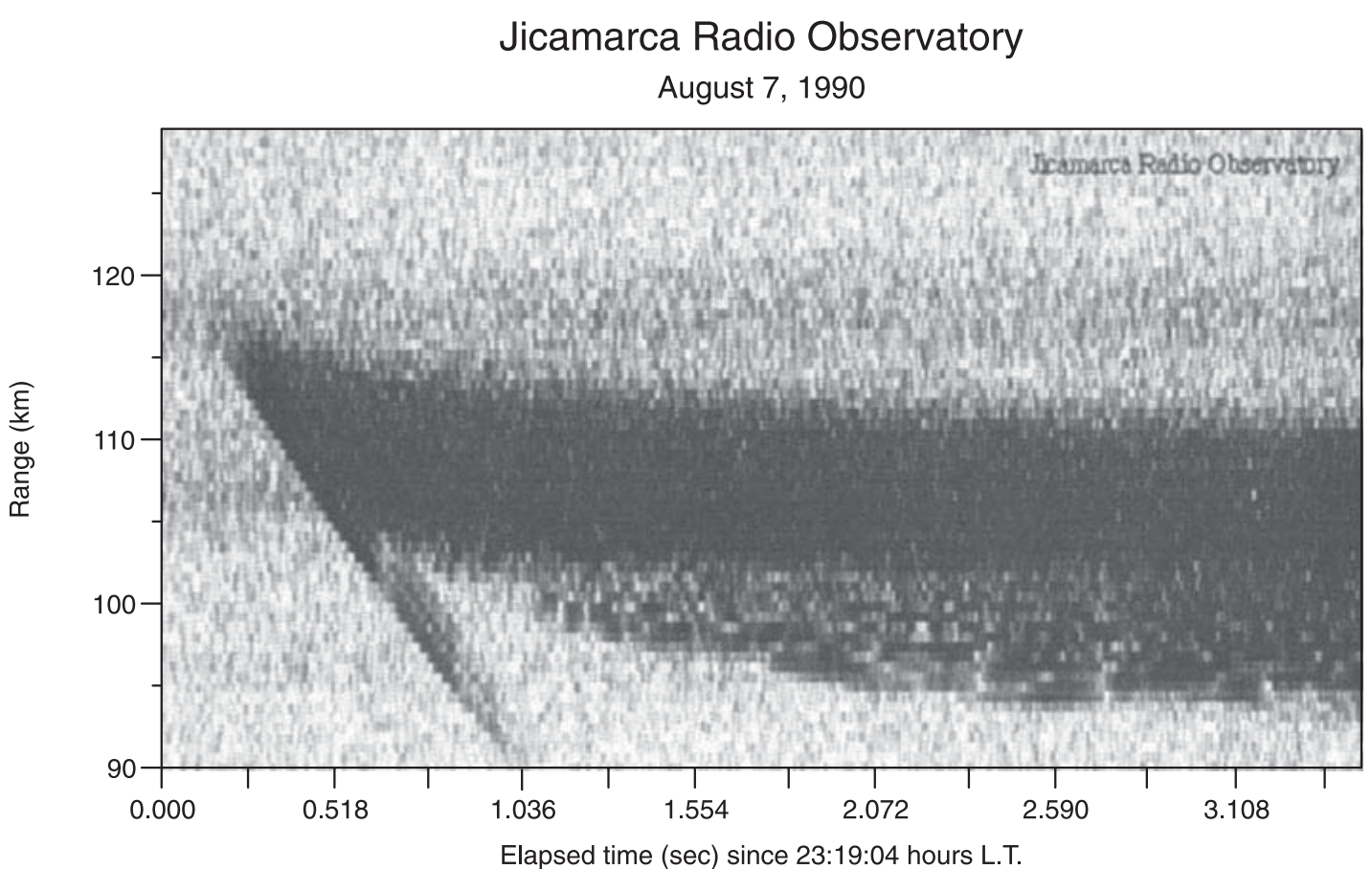

Fig. 6 Example of radar backscatter measurements of a meteor ablating in the upper atmosphere. The diagonal line on the left hand side corresponds to the head echo, which creates a plasma density envelope as the meteoroid penetrates the atmosphere. The broad scattering region to the right of the diagonal line corresponds to plasma irregularities in the meteor trail (Chapin and Kudeki 1994)

the upper atmosphere, their distributions are transformed by neutral wind shears to form "sporadic-E" layers, as discussed in Sect. 4, while others contribute to charged aerosols that are responsible for strong radar echoes at lower altitudes in the mesosphere. The long-lived metallic ions may also be transported over long distances via upper atmospheric winds and electric fields and have been measured by satellites at altitudes as high as $1000 \mathrm{~km}$ (Grebowsky and Aiken 2002), far from the altitudes where ablation is greatest-near and below $100 \mathrm{~km}$.

\subsection{Daily Variation of the Mid- and Low-Latitude Ionosphere}

Prior to discussing the coupling of the ionosphere to the neutral gas and its dynamics, model computations are provided to illustrate how the ionosphere varies, at least in general sense, with both local time and altitude. Such a representation is provided in Fig. 7 for both solar minimum and solar maximum conditions. These computations are from the 2011 International Reference Ionosphere (IRI) as discussed in Bilitza et al. (2011), and show conditions for all local times along the geomagnetic equator at the equinox.

The model ionosphere in Fig. 7 shows how the plasma densities are enhanced during solar maximum conditions for all local times, with the peak of ionization (the F2 peak) moving to significantly higher altitudes compared to solar minimum conditions. For both solar minimum and solar maximum, the ionosphere is considerably more dense at higher altitudes $(>250 \mathrm{~km}$ ) for most of the day and well into the night, exhibiting its lowest plasma number densities before dawn. The plasma density at the lower altitudes $(<200 \mathrm{~km})$ is very low during the night due to recombination losses of the dominant molecular ions. The apparent decrease in density of the base of the F-region after sunset, which is most pronounced during solar maximum, is due to vertical transport and is discussed in Sect. 4. 
Electron Density Along Magnetic Dip Equator

IRI-2007 Model
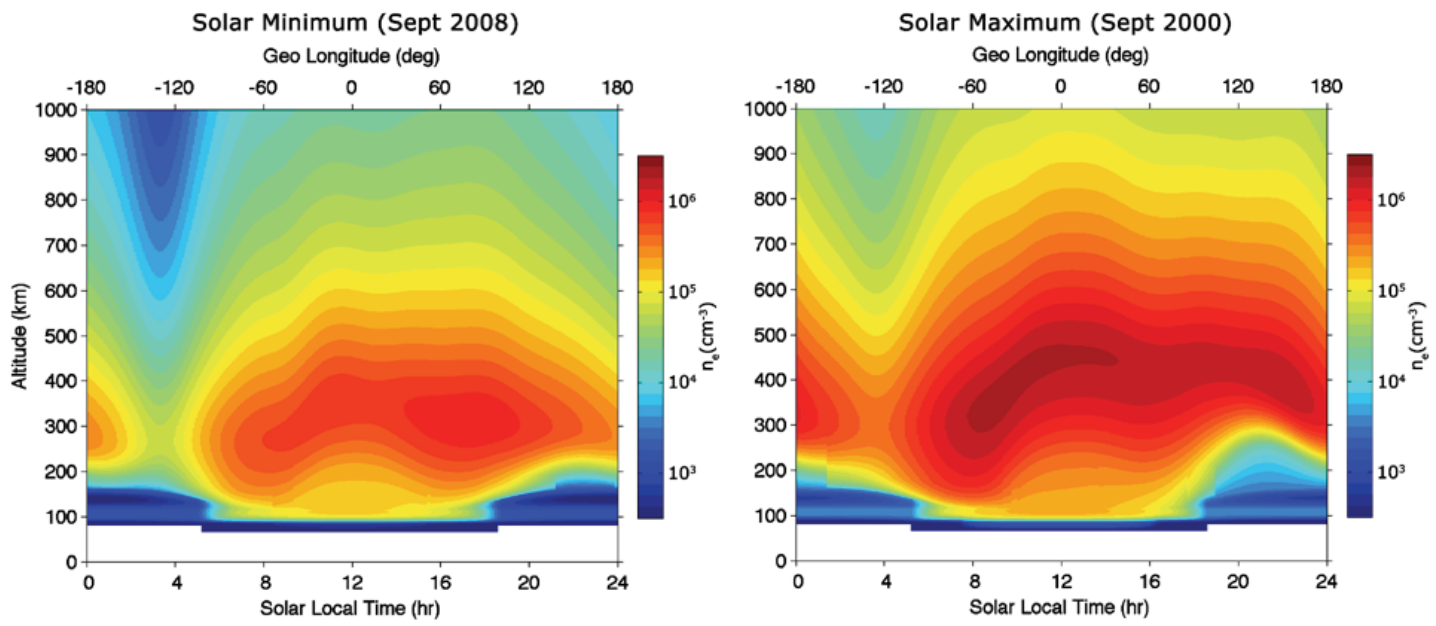

Fig. 7 IRI model calculations of electron density along the magnetic dip equator for 24 hours of local time at solar minimum (left) and solar maximum (right) conditions at the equinox

Another representation of this same equatorial plasma density distribution is shown in Fig. 8, in which the density is shown in a global distribution around the planet along the magnetic equator, again computed from the IRI. The panel depicts the ionosphere to $2000 \mathrm{~km}$ and is to scale with the earth's diameter. Seen as a narrow black circle, a very thin ionosphere-earth cavity (i.e., between the lower edge of the ionosphere and the earth's surface) is present, whose dimensions increase at night as the lower ionosphere goes away due to recombination effects, as discussed above. This cavity is particularly important for the excitation of Schumann resonances (low frequency electromagnetic waves that propagate around the globe) and other phenomena, as discussed by Simoes et al. (this issue).

To illustrate the diurnal variations of the ionosphere at a single location, the measured plasma density from $50-500 \mathrm{~km}$ is plotted for four consecutive days in Fig. 9 as observed by the Arecibo observatory in Puerto Rico (Figure courtesy N. Aponte). The day numbers at the bottom of the plot correspond to local noon. Clearly, the data show that the lower altitude $(<250 \mathrm{~km})$ ionosphere turns on very quickly after sunrise and then it recombines abruptly at sunset. During the night, the ionospheric density is hence substantially reduced at altitudes up to about $250 \mathrm{~km}$, where the lower edge of the F-region can be very "ragged", influenced perhaps by transport effects. During the day, the F-region plasma density is fairly uniform, extending into nighttime where it begins to decrease at the higher altitudes after midnight. The very faint, thin lines visible at night near $110 \mathrm{~km}$ are due to sporadic-E layers and will be discussed further on below.

Finally, we show IRI model computations illustrating how the plasma temperature varies with local time and altitude in Fig. 10. Note how the electron temperature increases dramatically in the early morning, as discussed above.

\section{Coupling of the Near-Earth Plasma and the Neutral Atmosphere Gases}

In this section, we first describe how the ionized gases are organized by the ambient magnetic field and then discuss how the ionospheric charged constituents are coupled to the neutral gas via collisions, which vary markedly with altitude. We describe how this coupling is 


\section{Plasma Density at the Magnetic Equator - IRI-2007 Model 22 Sep 2004}
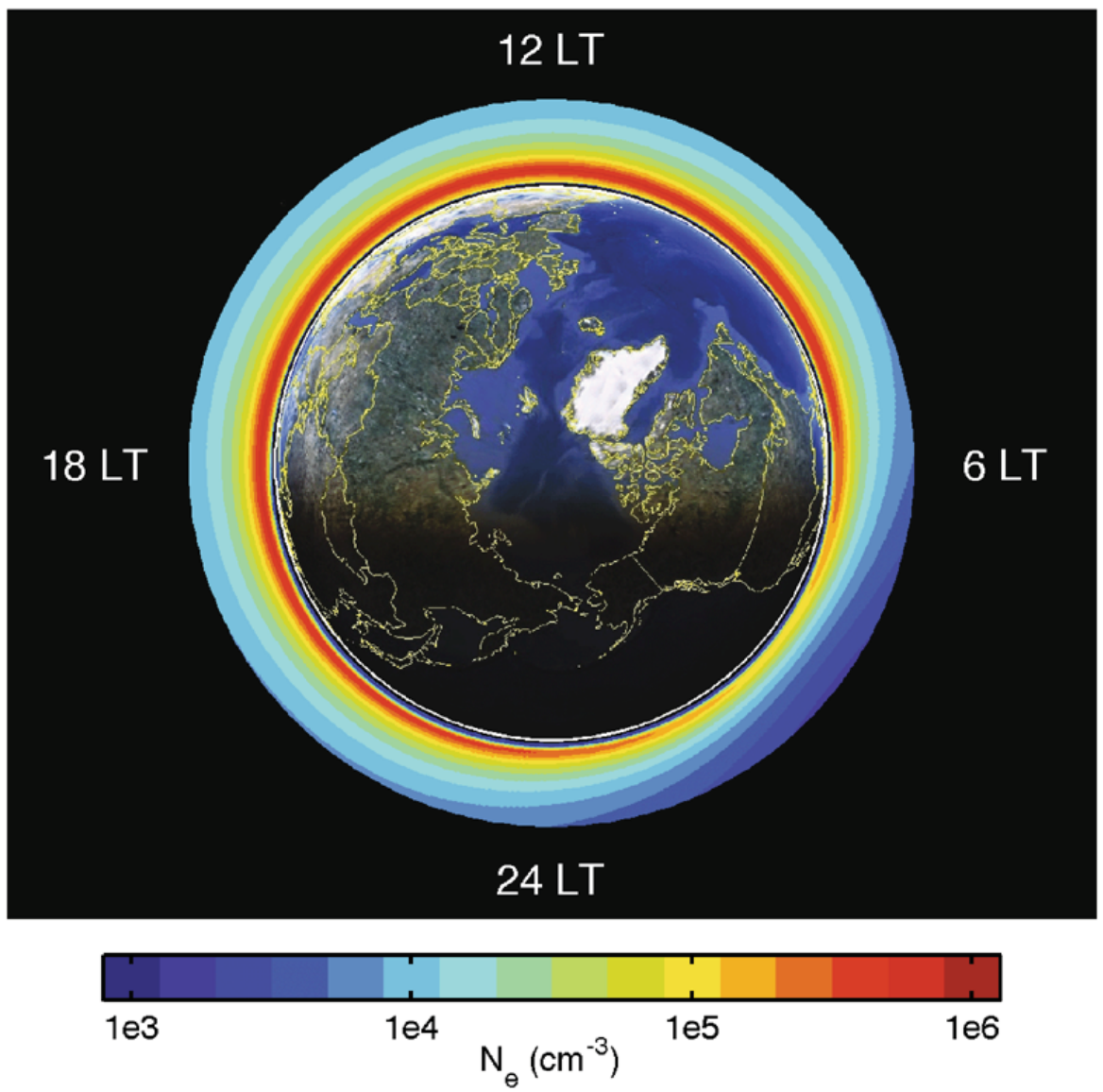

Fig. 8 IRI model calculations of the plasma density at the magnetic equator, presented in the altitude region scaled to the earth's radius

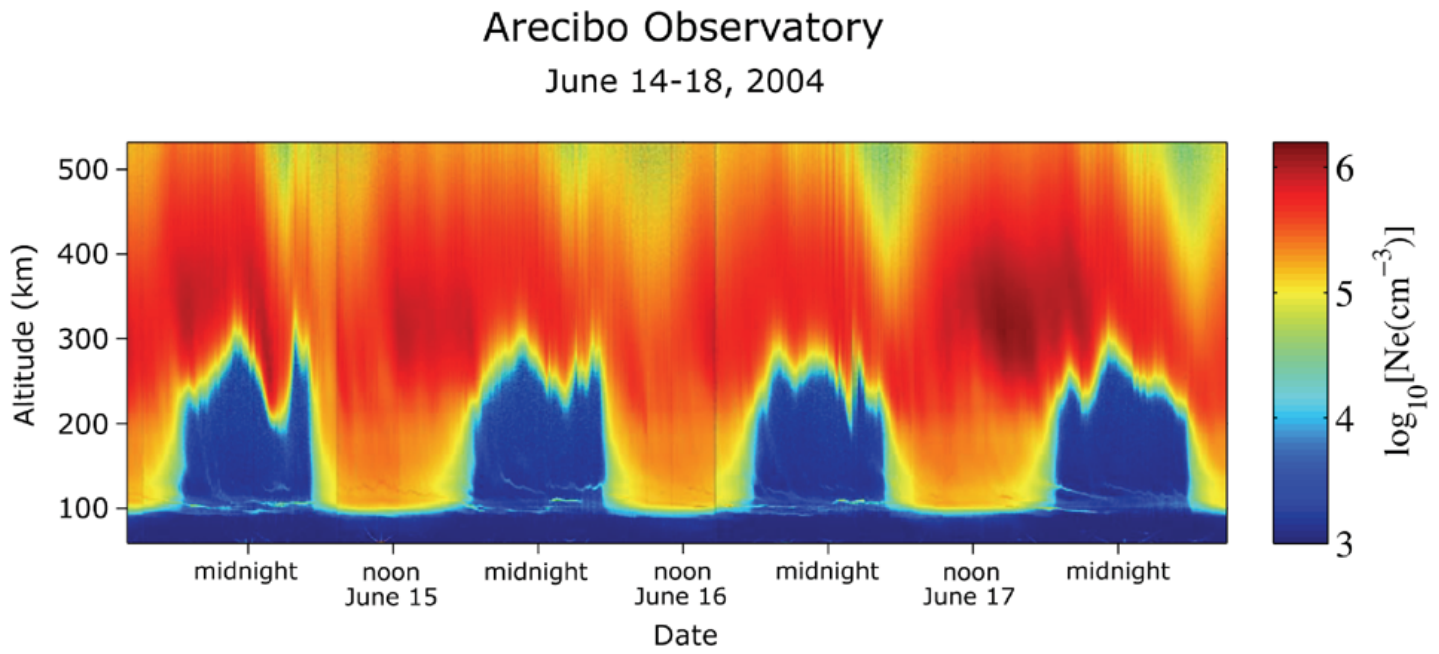

Fig. 9 Incoherent scatter measurements of the plasma density for four consecutive days at the Arecibo Observatory in Puerto Rico (Figure courtesy of N. Aponte) 
Fig. 10 IRI model calculations of the electron and ion temperatures along the magnetic equator for a 24 hour period
Plasma Temperatures Along Magnetic Dip Equator IRI-2007 Model, 22 Sep 2004

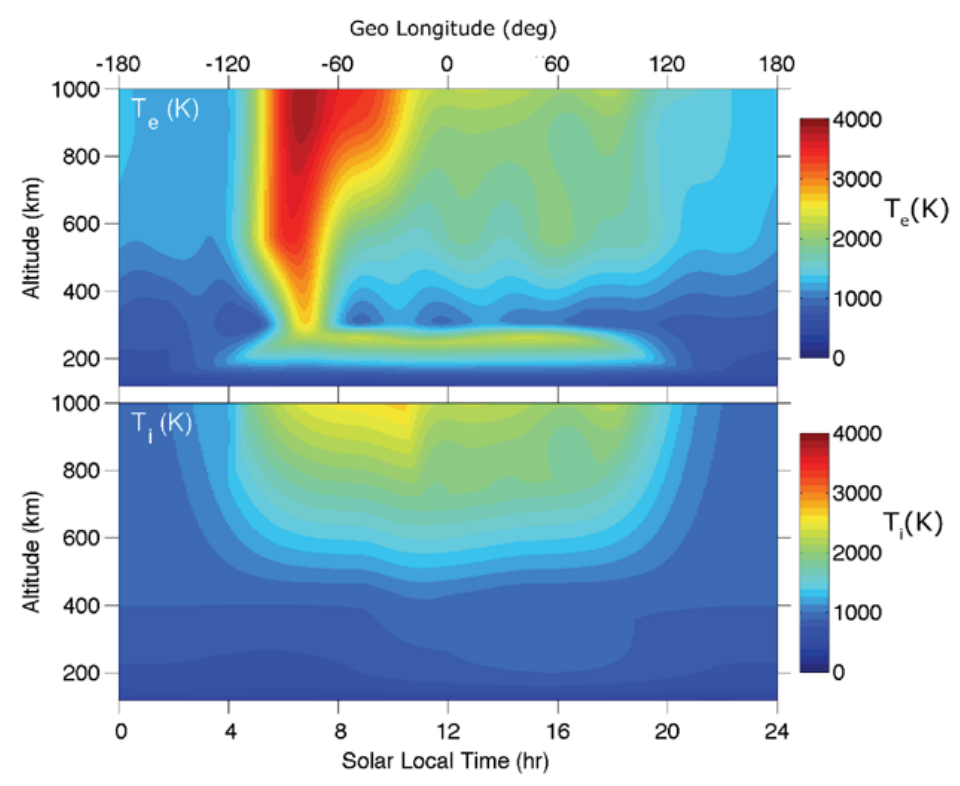

Fig. 11 The Earth's dipole magnetic field. The field lines are nearly vertical at very high latitudes yet nearly horizontal at low latitudes. The magnetic field line geometry has profound effects on the near-earth plasma. The magnetic poles are offset from the geographic poles

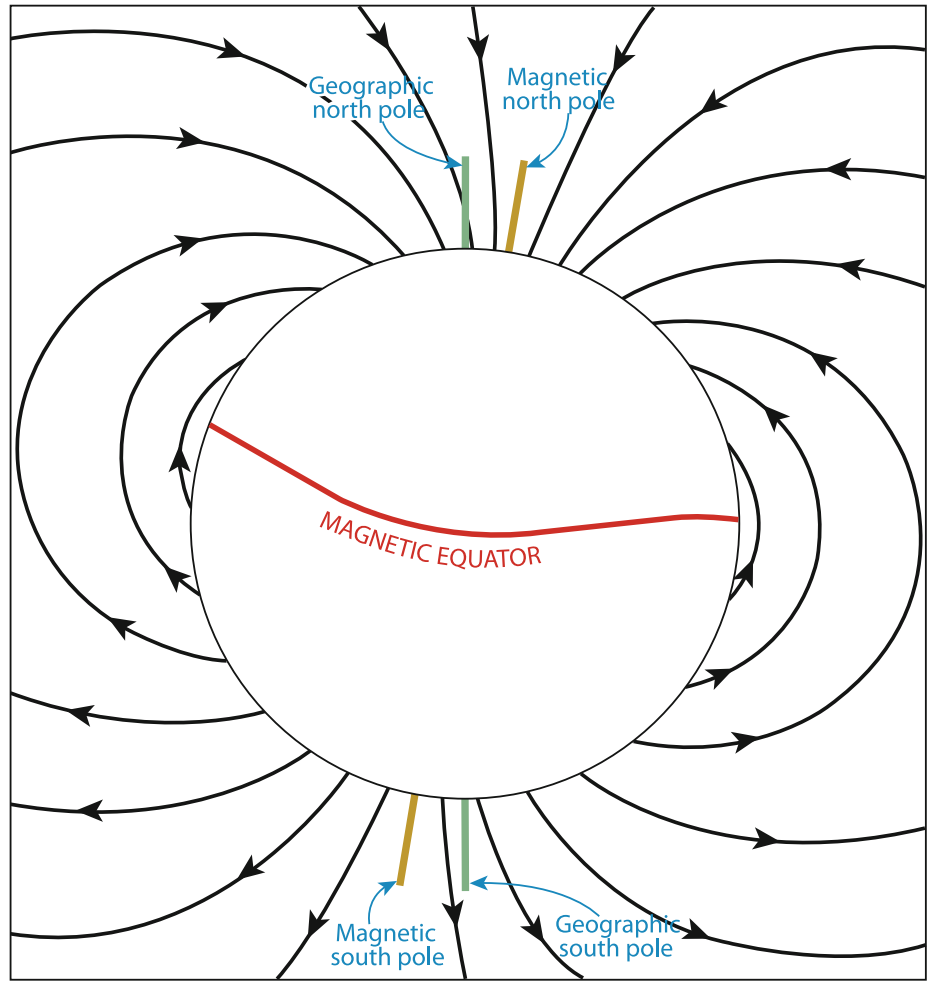

represented by the mobility and conductivity tensors, which form the basis of a large number of physical processes that govern the behavior of the partially ionized gas environment.

\subsection{The Earth's Magnetic Field}

Unlike the neutral atmospheric gases, the ions and electrons that comprise the near-earth plasma are compelled by Lorentz forces to gyrate about the earth's magnetic field lines. 


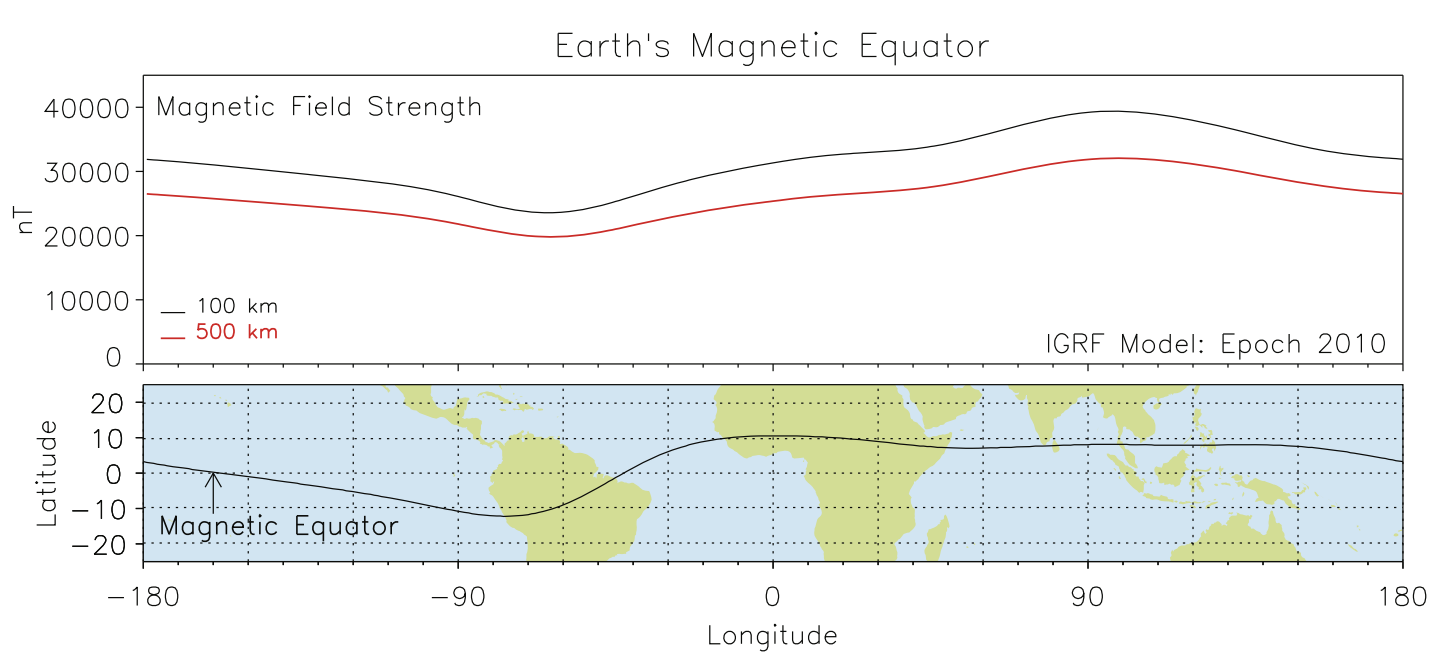

Fig. 12 The lower panel shows the location of the earth's magnetic equator. The upper panel shows the model magnetic field strength along the magnetic equator at $100 \mathrm{~km}$ and $500 \mathrm{~km}$ altitude

Thus, an appreciation of the earth's magnetic field is essential to understand the behavior of the near-earth plasma.

The Earth's magnetic field is essentially that of a dipole whose axis is tilted with respect to the earth's axis of rotation by approximately 10 degrees, as shown in Fig. 11. Consequently, the magnetic equator is also offset from the geographic equator. This figure shows how the main field lines are nearly vertical at high latitudes, stretching into the deep magnetosphere, yet remain closer to the earth, subtending much smaller angles with respect to the horizontal below about 45 degrees latitude. Indeed, at latitudes of roughly 60 degrees and below, the field lines "close", meaning that they extend to the conjugate hemisphere at roughly the same magnetic latitude. This condition strongly influences the near-earth plasma, as we shall show.

The magnetic field strength at $100 \mathrm{~km}$ altitude near the poles is $\sim 0.65 \mathrm{G}$, and decreases to about half that value at the magnetic equator, which is defined by the location on the earth where the magnetic field vector is strictly horizontal. In addition to being tilted, the dipole is slightly distorted and hence the magnetic field strength near $100 \mathrm{~km}$ altitude may be as low as $0.25 \mathrm{G}$ in the Peruvian sector but near $0.4 \mathrm{G}$ in the Indian sector at the magnetic equator. These differences strongly influence currents and longitudinal effects of plasma flow. Figure 12 shows the geographic location of the model magnetic equator, as well as the strength of the magnetic field at the equator. The declination of the magnetic field at the equator is generally perpendicular to the magnetic equatorial plane. This declination introduces longitudinal influences on plasma drifts and can also be important for magnetic flux tubes whose "footpoints" in the northern and southern hemispheres may be significantly displaced in longitude.

As we shall show, the earth's intrinsic magnetic field has a profound effect on the plasma with respect to directing $\mathbf{E} \times \mathbf{B}$ drifts and currents, as well as connecting the near-earth plasma with energy and momentum sources in the distant magnetosphere.

\subsection{Coupling of the Ionized and Atmospheric Gases: Collisions and the Mobility and Conductivity Tensors}

Overview. We demonstrated in the previous section how the ionized and atmospheric gases co-exist in the same volume particularly in the altitude range of 90-1000 km. We now discuss how the ionized and neutral gases are coupled, which sets the stage for later sections 
Fig. 13 Altitude profiles of the electron-neutral collision frequency and the ion-neutral collision frequency. Profiles of the mean ion gyrofrequency and electron gyrofrequency are also shown

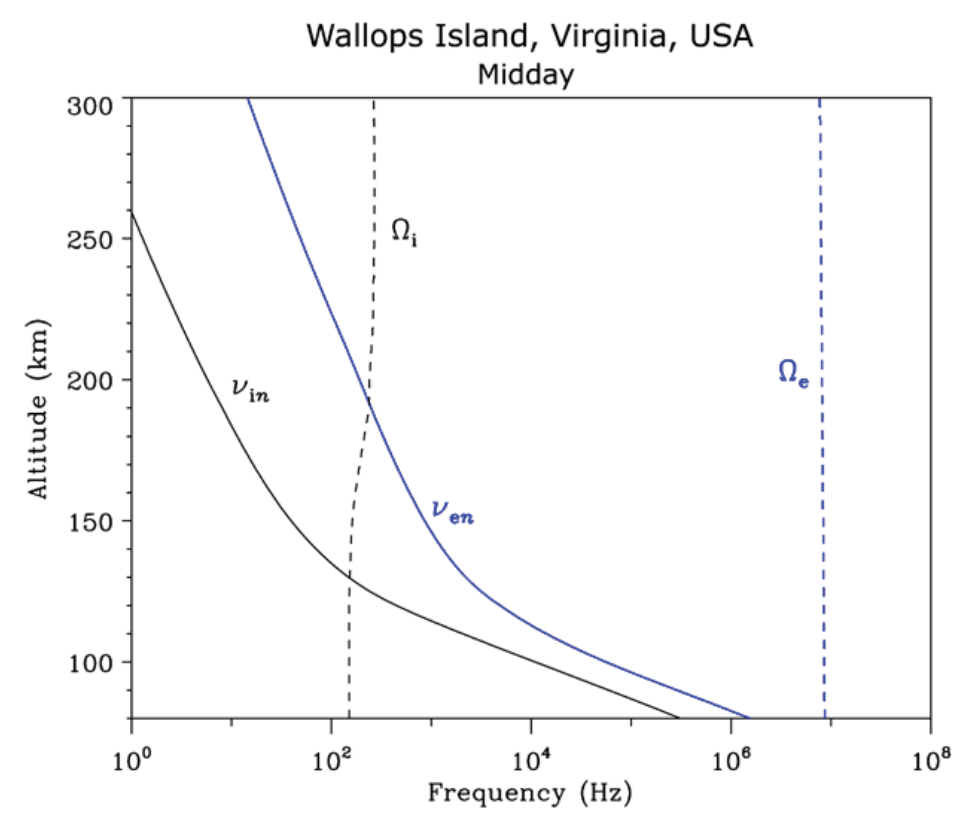

where we show how the partially ionized fluid responds to different forcing functions, primarily those due to electric fields and winds. In particular, we demonstrate that, although the ionized component is controlled by the earth's magnetic field, both the ionized and neutral gas motions are subject to collisional interactions between the ions and neutrals that vary with altitude. These interactions are specified by the mobility and conductivity tensors, as shown below.

Gyro and Collision Frequencies. Charged particles in the ionosphere gyrate about the ambient magnetic field lines at their cyclotron frequencies, $\Omega_{j}$, given by

$$
\Omega_{j}=e|\mathbf{B}| / m_{j}
$$

where $e$ is the electric charge, $|\mathbf{B}|$ is the magnetic field strength, and $m_{j}$ is the mass of the species which is designated by $j$. For reference, the gyro frequency for $\mathrm{O}^{+}$ions in a magnetic field of 0.5 Gauss is $48 \mathrm{~Hz}$, whereas the gyro frequency for electrons is $1.4 \mathrm{MHz}$ for the same magnetic field strength.

Since the upper atmospheric gases are dense (compared to the plasma) and only weakly ionized, collisions between ion and neutrals significantly influence the individual and bulk motions of the plasma. Because of these collisional effects, the statistical collective behavior of the particles may be defined using the equations of hydrodynamics. Various expressions have been put forward for the ion-neutral collision frequency, $v_{i n}$, and the electron-neutral collision frequency, $v_{e n}$, that depend on the neutral density, neutral and ion masses, and the temperatures (e.g., Banks and Kockarts 1973).

For the lower altitude regions of the ionosphere, model ion-neutral and electron-neutral collision frequency profiles are shown in Fig. 13 for a mid-latitude location at mid-day. It is clear that below $130 \mathrm{~km}$, the ions are collision dominated by the atmosphere whereas the electrons are able to execute their gyro motions about the magnetic fields and consequently undergo $\mathbf{E} \times \mathbf{B}$ drifts across magnetic field lines. The lower ionosphere, where $v_{i n} / \Omega_{i}>1$ and $v_{e n} / \Omega_{e} \ll 1$, constitutes the dynamo region where strong currents flow, as discussed in the next section. 
Mobility and Conductivity Tensors. Because the plasma distribution function in the presence of a magnetic field is highly anisotropic, we define a mobility tensor, $\overline{\overline{\boldsymbol{\mu}}}$, which relates the velocity $(\mathbf{V})$ and force $(\mathbf{F})$ vectors:

$$
\mathbf{V}=\overline{\overline{\boldsymbol{\mu}}} \cdot \mathbf{F}
$$

where

$$
\overline{\overline{\boldsymbol{\mu}}}=\left[\begin{array}{ccc}
\mu_{1} & \mu_{2} & 0 \\
-\mu_{2} & \mu_{1} & 0 \\
0 & 0 & \mu_{0}
\end{array}\right]
$$

This tensor has been defined in a geometry such that the magnetic field, $\mathbf{B}$, is parallel to the z-axis. For single particle motion subject to an applied force, we have the following mobilities:

$$
\begin{aligned}
& \mu_{o j}=1 / m v_{j n} \quad(\text { parallel mobility) } \\
& \mu_{1 j}=\left(1 / m v_{j n}\right)\left(v_{j n}\right)^{2} /\left(\Omega_{j}^{2}+v_{j n}^{2}\right) \quad \text { (Pedersen mobility) } \\
& \mu_{2 j}=\left(1 / m v_{j n}\right)\left(v_{j n} \Omega_{i}\right) /\left(\Omega_{j}^{2}+v_{j n}^{2}\right) \quad \text { (Hall mobility) }
\end{aligned}
$$

The profiles of these three mobility components are plotted in Fig. 14 for three different locations, one at the magnetic equator (Jicamarca, Peru), one at high latitudes (Poker Flat, USA), and one at mid-latitudes (Wallops Island, USA). The mobilities shown here are combined for ions and electrons, and use the mean molecular weight for the ions. Note that the Pedersen mobility peaks at around $125 \mathrm{~km}$ and the Hall mobility peaks near $105 \mathrm{~km}$. The mobilities are higher at the lower latitudes due to the weaker magnetic field strength, since they are roughly proportional to $\sim 1 / \Omega_{i}^{2}$.

We now define a conductivity tensor, $\overline{\bar{\sigma}}$, that relates the electric field and current density:

$$
\mathbf{J}=\overline{\bar{\sigma}} \cdot \mathbf{E}
$$

which can also be expressed in terms of the difference of the ion and electron motions:

$$
\mathbf{J}=N e\left(\mathbf{V}_{i}-\mathbf{V}_{e}\right),
$$

where $N$ is the plasma density and $e$ is the electric charge.

The conductivity may be expressed in terms of the mobilities such that $\sigma=$ $N e^{2}\left(\boldsymbol{\mu}_{i}+\boldsymbol{\mu}_{e}\right)$. The components of the conductivity are thus:

$$
\begin{array}{ll}
\sigma_{o}=N e^{2}\left(\mu_{o e}+\mu_{o i}\right) & \text { (parallel conductivity) } \\
\sigma_{1}=N e^{2}\left(\mu_{1 e}+\mu_{1 i}\right) & \text { (Pedersen conductivity) } \\
\sigma_{2}=N e^{2}\left(\mu_{2 e}-\mu_{2 i}\right) & \text { (Hall conductivity) }
\end{array}
$$

The ionospheric conductivity depends linearly on the ambient plasma density and the coupled neutral and ionized gases via the anisotropic mobilities as organized by the magnetic field. Equation (4) may now be expressed more fully:

$$
\mathbf{J}=\sigma_{1} \mathbf{E}_{\text {perp }}^{\prime}+\sigma_{2} \mathbf{b}^{\prime} \times \mathbf{E}_{\text {perp }}^{\prime}+\sigma_{o} \mathbf{E}_{\text {par }}
$$

where $\mathbf{b}^{\prime}$ is the unit vector along the magnetic field direction and $\mathbf{E}_{\text {perp }}^{\prime}$ is the perpendicular electric field in the earth-fixed frame, such that $\mathbf{E}_{\text {perp }}^{\prime}=\mathbf{E}_{\text {perp }}+\mathbf{U} \times \mathbf{B}$, where $\mathbf{U}$ is the neutral wind. This expression shows that if there is an electric field perpendicular to the magnetic 

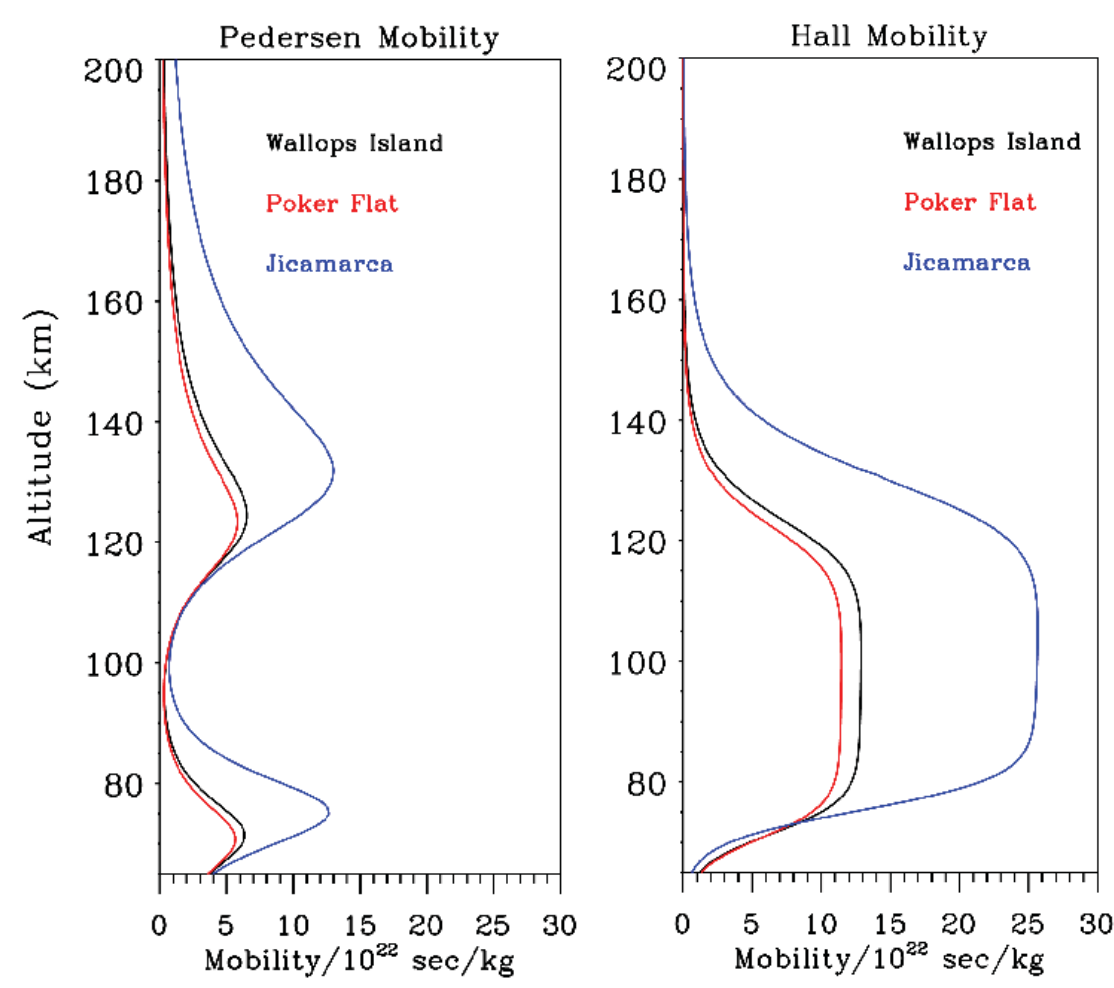

Fig. 14 Altitude profiles of the Pedersen and Hall mobilities at Wallops Island, Virginia; Poker Flat, Alaska; and Jicamarca, Peru

field, $\mathbf{B}$, the current will have two components-one along the electric field but perpendicular to $\mathbf{B}$ (Pedersen) and one perpendicular to both the electric field and to $\mathbf{B}$ (Hall). Their magnitudes differ by $v_{i} / \Omega_{i}$ and the angle, $\alpha$, between them is given by $\alpha=\tan ^{-1}\left(\Omega_{i} / v_{i}\right)$.

Model calculations of the Hall and Pedersen conductivity in the E-region at different latitudes are presented in Fig. 15. (The parallel conductivity for all locations is orders of magnitude higher than that of the corresponding Hall and Pedersen conductivity and is not shown in the figure.) The top two panels show the plasma density at noon and the Hall and Pedersen conductivities calculated using the mobilities as shown in Fig. 14. The lower panels show the plasma density and conductivity at 2100 LT for Poker Flat, Alaska for both quiet (left) and active (right) conditions. The active auroral conditions were selected to provide plasma densities of $10^{5} / \mathrm{cm}^{3}$ at $110 \mathrm{~km}$, corresponding to a moderate auroral arc. Clearly, for conditions where there is no aurora, the high latitude conductivity at night is very low. The thermal plasma created by the auroral particles provides a very strong conductivity which is important for current closure, Joule heating, and other important high latitude effects, as we will discuss later on.

\section{The Dynamics and Morphology of the Ionosphere at Low and Middle Latitudes}

In Sect. 2, we showed how the earth's ionosphere at low and middle latitudes is formed via solar photo-ionization and subsequently varies with altitude and local time, revealing important differences between the daytime and nighttime plasma number density and temperature. In this section, we show how this ionization is significantly altered by dynamics. The dynamics are driven primarily by neutral forcing (winds) of the ions that subsequently set up internal polarization electric fields. In the next section, we will show how the opposite 

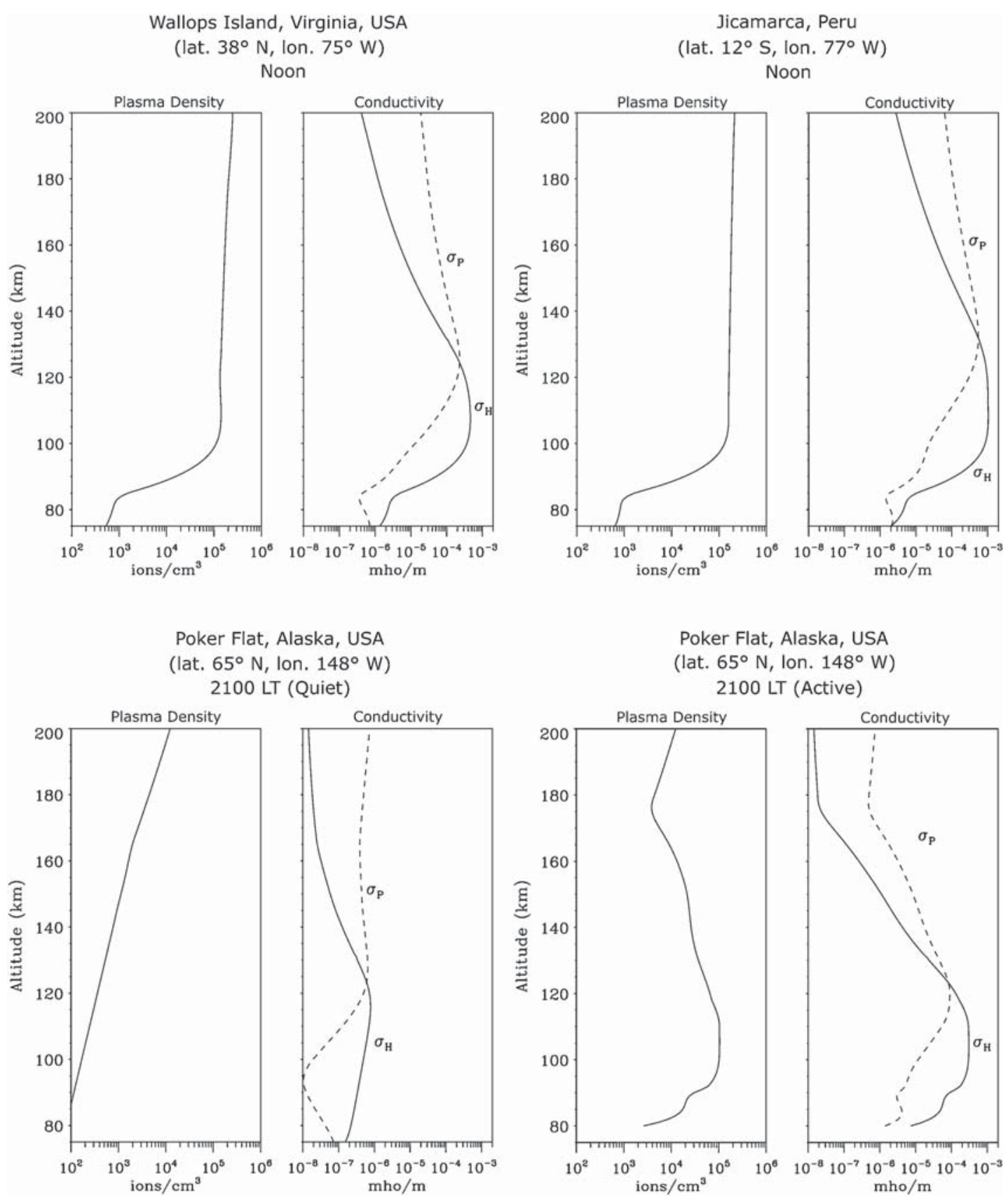

Fig. 15 Altitude profiles of the Hall and Pedersen conductivities for representative plasma density profiles at Wallops Island, Virginia, USA (upper left), and Jicamarca, Peru (upper right). The lower panels show the Hall and Pedersen conductivities at Poker Flat, Alaska, USA, for quiet conditions (left) and moderate auroral conditions (right)

occurs at high latitudes, i.e., electric fields drive $\mathbf{E} \times \mathbf{B}$ plasma motion via large amplitude, externally imposed, magnetospheric fields that, through ion-neutral collisions, subsequently drive neutral winds.

\subsection{Dynamo Currents and the Equatorial Electrojet}

As the solar EUV heats the neutral upper atmosphere, the neutral gases generally expand away from the sub-solar point, creating a system of upper atmosphere tidal motions or winds. 

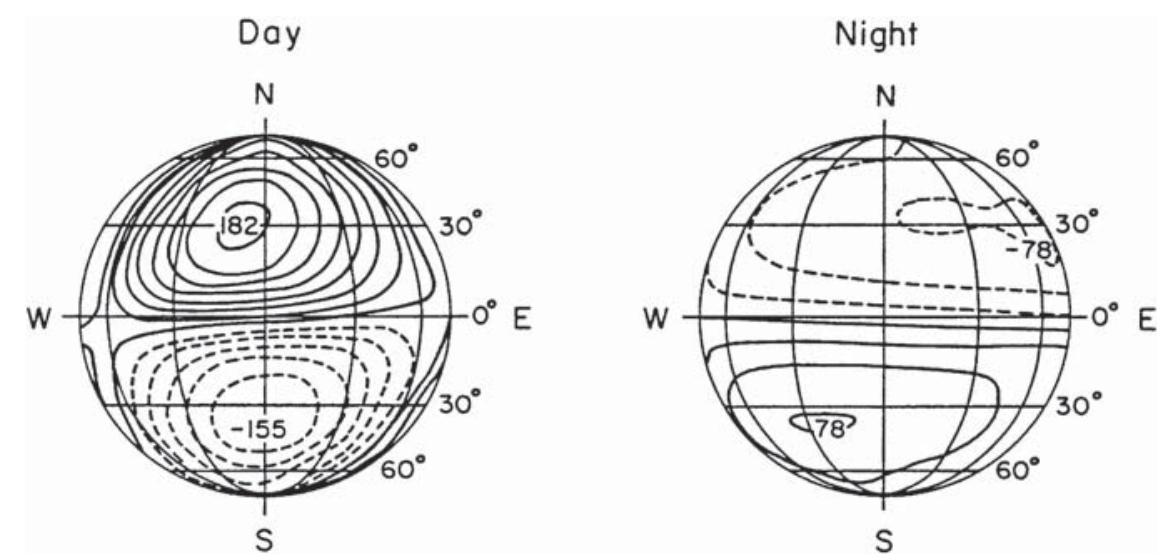

Fig. 16 Current patterns set up by the atmospheric dynamo for daytime (left) and nighttime (right) hemispheres (after Matsushita 1965)

The neutral motions drag ions across magnetic field lines via ion-neutral collisions, creating a very small charge displacement with the electrons that subsequently sets up relatively weak, ionospheric electric fields. At the lower altitudes $(\lesssim 130 \mathrm{~km})$, the ion-neutral collision frequencies become so large that the ions are unable to complete their gyro orbits whereas the electron cyclotron orbits, due to their much smaller mass, are not restricted and hence, remain "magnetized". Due to the differential drifts of the ions and electrons at these low altitudes, a system of global scale, horizontal current patterns develops, in which internal, polarization electric fields are generated to restrict the current flow to be non-divergent. This worldwide system of currents is called the "solar quiet" (or Sq) dynamo which form current loops at mid-latitudes which close at the magnetic equator, as shown in Fig. 16 (Matsushita 1965).

The strongest current driven by the atmospheric dynamo is the equatorial electrojet. This current system exists as a result of both the dynamo action at all latitudes and the enhanced conductivity set up by the horizontal magnetic field geometry at the magnetic dip equator. At this location, in the altitude range of roughly $95-115 \mathrm{~km}$, significant zonal (east-west) electron drifts are set up in the following way. Vertical $\mathbf{E} \times \mathbf{B}$ drifts due to the ambient eastward electric fields (driven by the solar forcing discussed above) combined with the north-south magnetic field are inhibited for ions because the ion-neutral collisions are sufficiently large such that $V_{i}=0$. Hence, only the electrons are magnetized and the resulting vertical charge separation between the electrons and ions establishes a vertical polarization field, $\mathbf{E}_{p}$. This polarization electric field, in turn, creates a horizontal $\mathbf{E}_{p} \times \mathbf{B}$ drift which reinforces the small, existing dynamo current and thus creates the strong electrojet current. This process is depicted in Fig. 17. The equatorial electrojet is strongest within a degree of the magnetic equator, where the magnetic field vector is precisely horizontal, thus enabling the vertical, polarization electric field described above to be set up. This current can also be explained in terms of the Cowling conductivity, which is an enhancement, by a factor of $\sigma_{2}^{2} / \sigma_{1}$, of the Pedersen conductivity at the magnetic equator (e.g., Kelley 2009).

At night, the zonal electric field reverses direction and thus the polarization electric field is downward. The resulting $\mathbf{E} \times \mathbf{B}$ drift again enhances the electrojet current since the current is also of opposite direction at night. The magnitude of the nighttime current densities is much lower than during the daytime due to the greatly reduced ambient electron density.

An example of measurements of the polarization electric field, current, and plasma density measured on a sounding rocket launched in the daytime electrojet from Brazil is shown in Fig. 18a. In this figure, the resulting electrojet plasma drifts are calculated independently 
Equatorial Electrojet
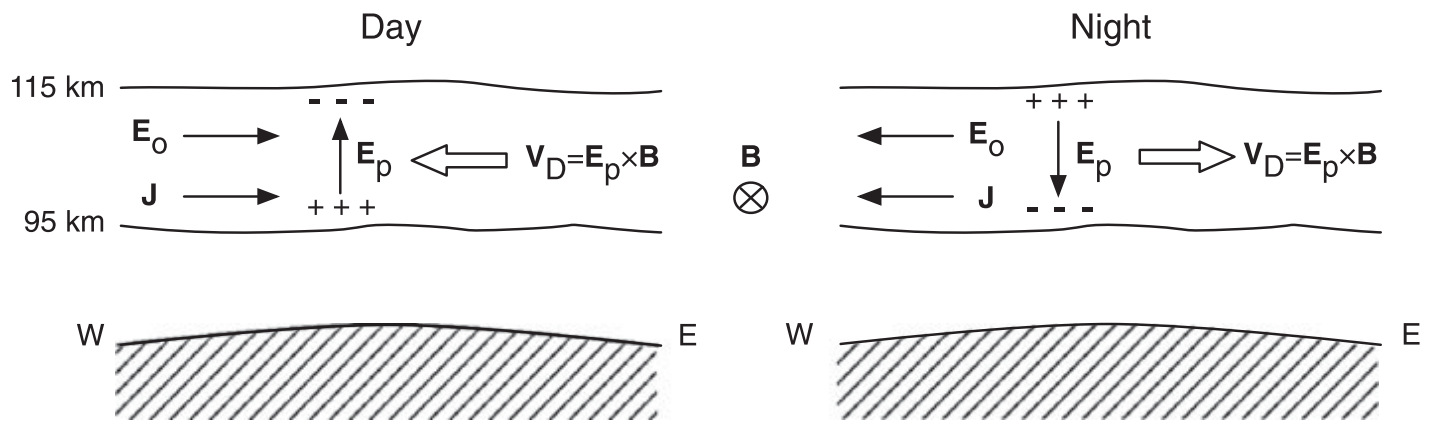

Fig. 17 The zonal electric fields create the vertical polarization fields $\left(\mathbf{E}_{p}\right)$ at the magnetic equator in daytime (left) and nighttime (right). The $\mathbf{E}_{p} \times \mathbf{B}$ drifts drive the daytime and nighttime electrojets in opposite directions in accordance with the sign of $\mathbf{E}_{p}$

using both the measured electric fields (i.e., the $\mathbf{E} \times \mathbf{B}$ drift) and the measured current and plasma density (i.e., $\mathbf{V}_{e}=\mathbf{J} / q N_{e}$, assuming $V_{i}=0$ ), which show comparable results (Pfaff et al. 1997a). The discrepancy between these two drift profiles may be due to the neutral wind contribution, which was not measured in this experiment and hence not taken into account.

When the relative electron-ion drift speed exceeds approximately the acoustic velocity, collisional two-stream waves are excited (Farley 1963; Buneman 1963). These intense, meter-scale waves are observed in electric field wave measurements recorded by sounding rockets, for example in the equatorial electrojet off the coasts of Peru and Brazil (Pfaff et al. 1987, 1997b). The altitude-spectrogram in Fig. 18b shows an example of such two-stream waves, represented by the broadband emissions extending to higher frequencies between 102 and $110 \mathrm{~km}$, where the instability threshold conditions are met. The intense, lower frequency waves observed in particular at the lower altitudes are driven by the gradient-drift instability and are set up by the vertical (positive upwards) DC electric field in concert with the upward ambient plasma gradient. The lower edge of the daytime ionosphere is unstable at long wavelengths in the electrojet region, as evidenced by structure in the plasma density profile at $105 \mathrm{~km}$ altitude and below, shown in the figure and discussed in Pfaff et al. (1987). Indeed, the lower ionosphere in both the daytime and nighttime electrojet regions is typically highly unstable to these plasma instabilities (e.g., Fejer et al. 1975). Distinct, kmscale waves dominate the spectrum when the gradient-drift instability is active, propagating zonally along the electrojet direction. These large scale waves have been shown to change directions between daytime and nighttime conditions as the ambient polarization electric field changes sign (Kudeki et al. 1982, 1987).

\subsection{The Fountain Effect: The F-region Dynamo, and Plasma Drift Patterns at Low Latitudes}

Given the formation of the ionosphere by photo-ionization from solar photons and the controlling role of the solar zenith angle in the resulting plasma production, it would be logical to expect the ionosphere to have maximum number density at the sub-solar point and that the variations in this density would be fairly predictable with local time, latitude, and season. In fact, this is not the case. The daytime ionospheric density does not peak at the sub-solar point, but actually has two distinct maxima about \pm 15 degrees from the magnetic equator which can be quite pronounced. 


\section{NASA 21.111 (Downleg) -- Alcantara, Brazil \\ 21 September 1994 10:56:00 L.T.}
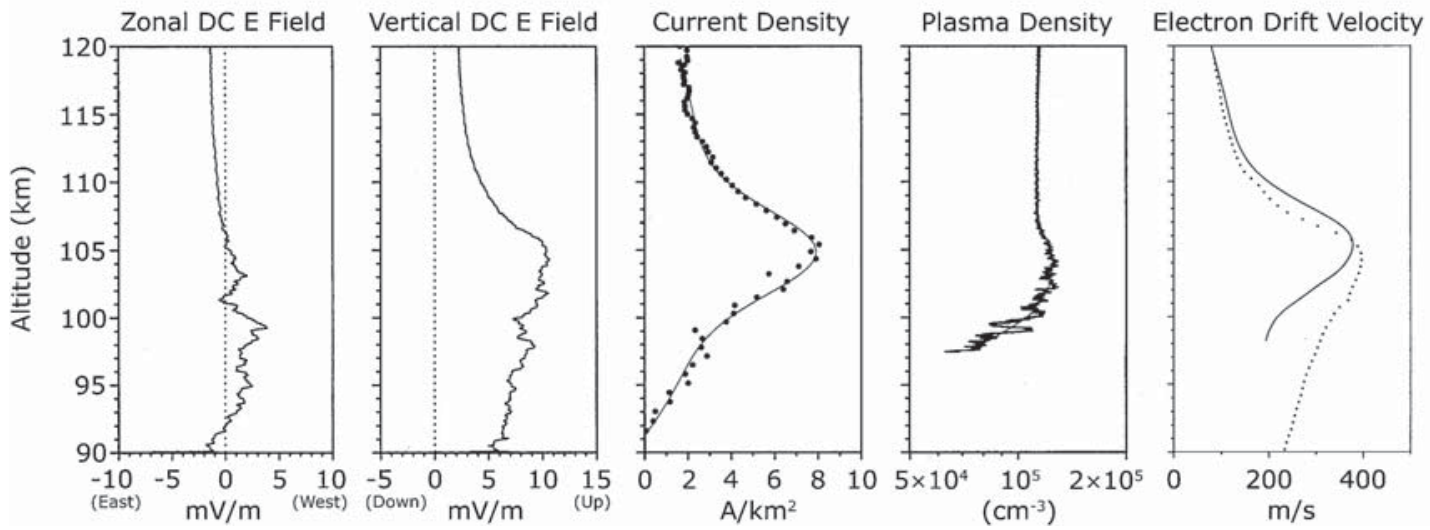

(a)

\section{Flight 33.027 -- Punta Lobos, Peru \\ March 12, 1983 -- 10:35 L.T.}

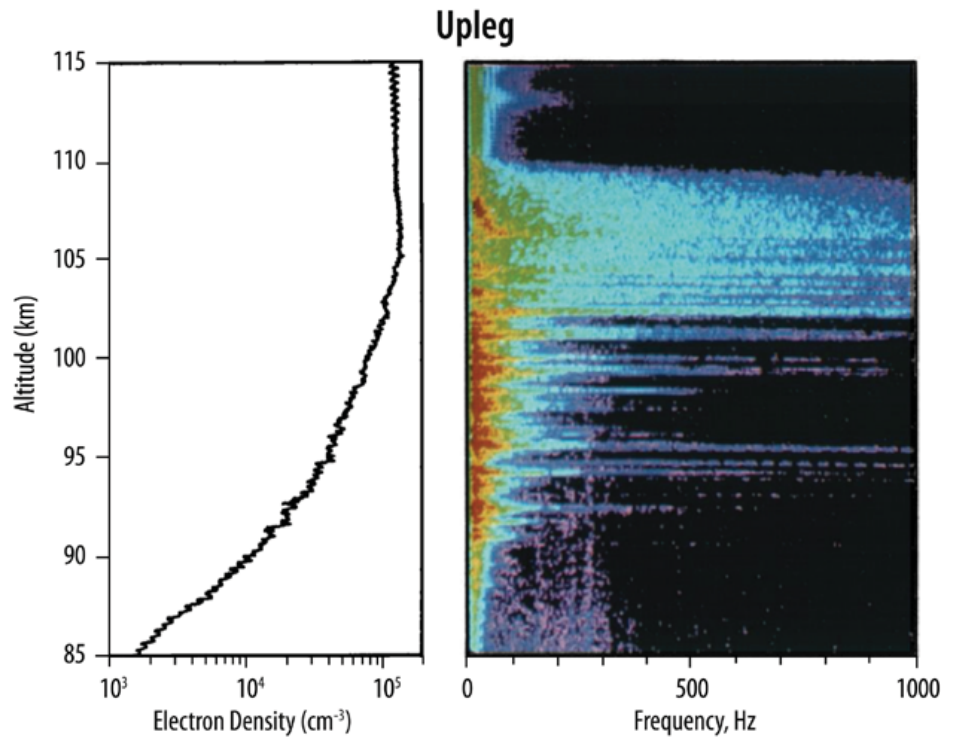

(b)

Fig. 18 (a) Sounding rocket observations of the altitude profile of the DC electric field, current density and plasma density in the daytime equatorial electrojet at Alcântara, Brazil. The panel on the right shows independent calculations of the electron drift velocity using (solid) the current density and plasma density and (dotted) the polarization electric field (Pfaff et al. 1997b). (b) Sounding rocket observations of electric field waves displayed in an altitude sonogram (right panel) in the daytime equatorial electrojet at Punta Lobos, Peru. The color levels correspond to over $40 \mathrm{~dB}$ in wave power, with red denoting stronger amplitudes than blue. Simultaneous measurements of the unstable plasma density are shown in the left hand panel (Pfaff et al. 1987)

These ionization peaks and the equatorial valley in between them were initially observed in ionosonde data and eventually explained as a consequence of the eastward electric field in the daytime, low latitude ionosphere (Appleton 1946; Martyn 1947). As a result of this electric field, the $\mathbf{E} \times \mathbf{B}$ drift forces plasma upwards at the magnetic equator which then, via diffusion and gravity, "slides" down the magnetic field lines to form the two maxima away from the magnetic equator, peaking at $\sim 300-400 \mathrm{~km}$ altitude, as illustrated in Fig. 19. 


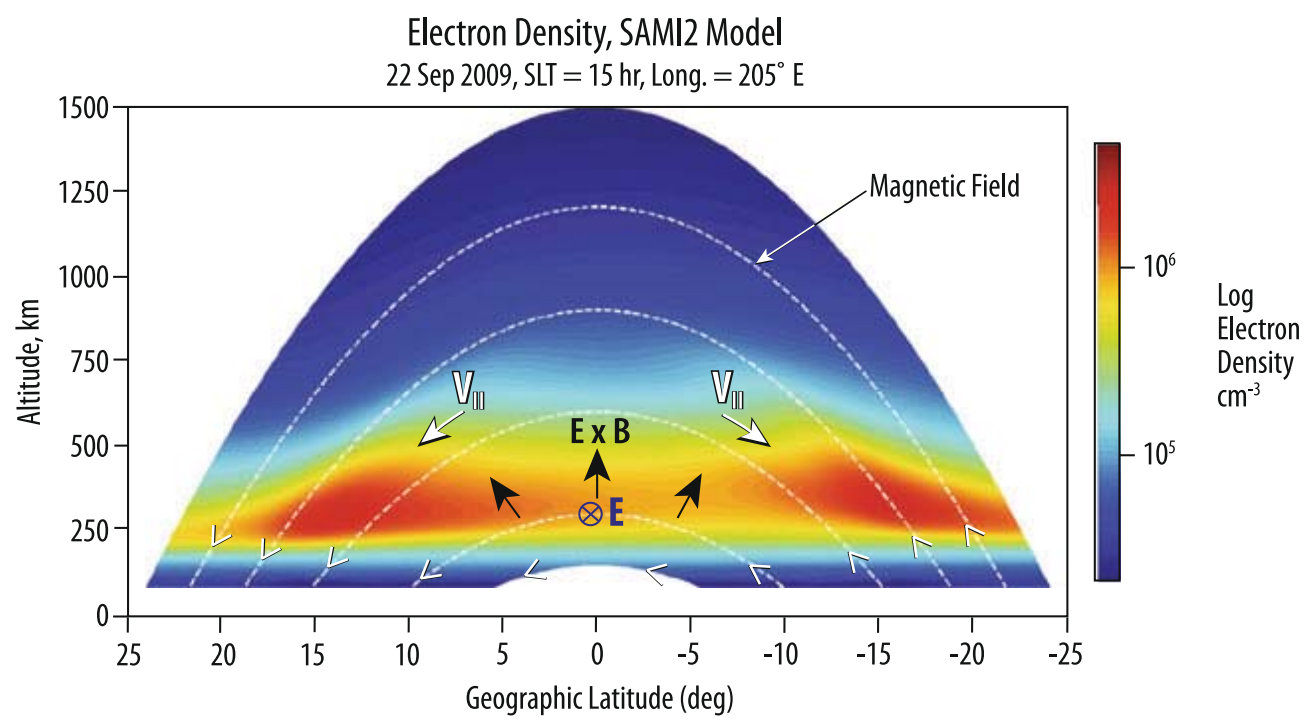

Fig. 19 SAMI2 model calculations versus latitude and altitude of the plasma density for 1500 SLT at $205^{\circ}$ East. The upward $\mathbf{E} \times \mathbf{B}$ drift at the magnetic equator is driven by the eastward electric field, and there is subsequent flow downward along the magnetic field lines

Fig. 20 SAMI2 model calculations of the global total electron content (TEC) at 22:32 UT, showing the equatorial anomaly enhancement in the late afternoon. The center of the globe corresponds to 17:44 LT (Figure courtesy J. Huba)

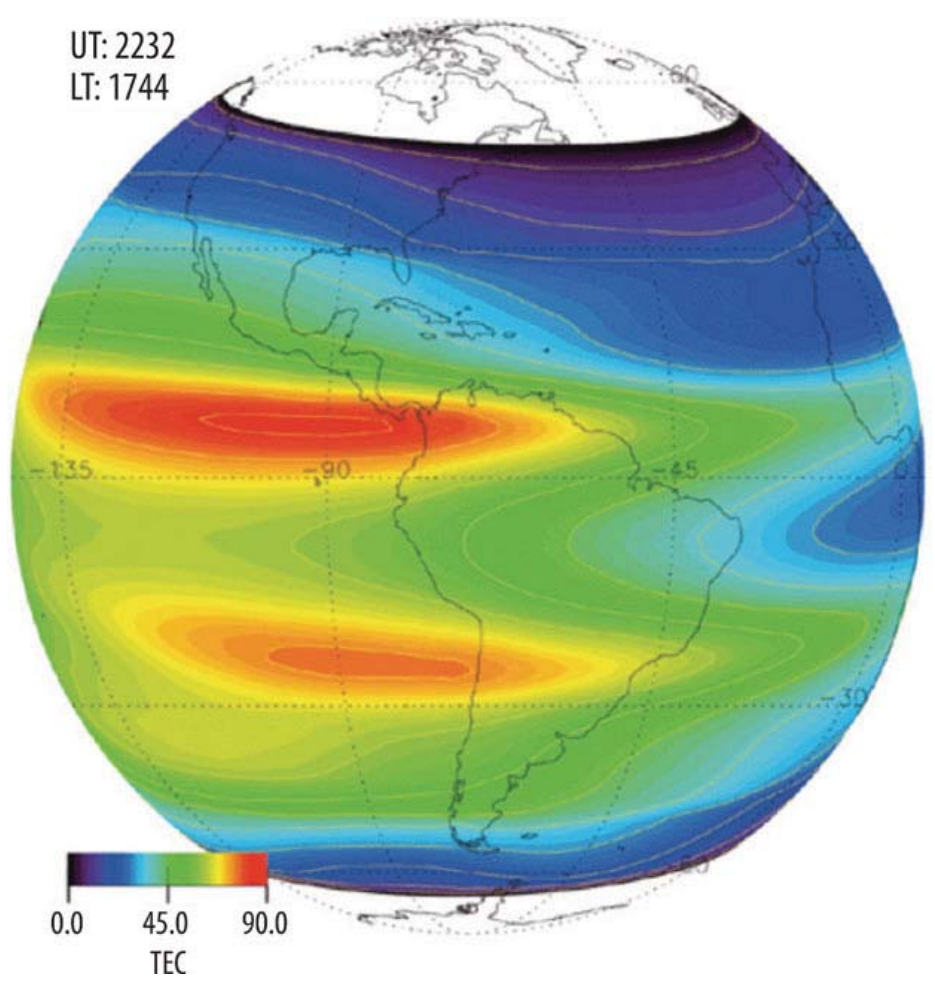

Referred to as the so-called "fountain effect" or the equatorial ionization anomaly (EIA), this phenomenon represents a significant, fundamental mechanism that characterizes the near-earth plasma environment. Model total electron content calculations for moderate solar conditions are shown in Fig. 20 that were calculated with the SAMI2 model (Huba et al. 2000) and show how the anomaly "wings" dominate the late afternoon ionosphere at low latitudes.

Another process at work at low latitudes that drives the plasma is the so-called "F-region dynamo". At high altitudes in the low latitude F-region during the daytime, wind-driven field aligned currents flow along the magnetic field lines between the F and E-regions. At 
night, however, the E-region ionosphere is devoid of plasma due to recombination effects discussed previously, and hence, the "feet" of the magnetic field lines, whose apex altitudes are in the F-region, are no longer in high conductivity E-region. As a result, large-scale electric fields are set up in the F-region which drive a low-latitude dynamo in the F-region at night in order to maintain current continuity (e.g., Rishbeth 1971, 1997; Heelis 2004).

An enhanced vertical drift in the F-region after sunset is a characteristic of the low latitude ionosphere, whose intensity exhibits seasonal, longitudinal, and solar cycle dependencies, tending to be largest near the equinoxes at solar maximum (Scherliess and Fejer 1999). This "pre-reversal enhancement" has been attributed to processes also related to differences in the conductivity between the E- and F-regions near the terminator and is generally attributed to a polarization electric field set up to maintain current continuity near the dusk meridian (e.g., Heelis et al. 1974; Farley et al. 1986; Haerendel and Eccles 1992; Eccles 1998).

Although the winds and polarization electric fields can be quite variable day-to-day, an average statistical pattern emerges from in situ and radar measurements of meridional (radially outward) drifts at low latitudes which is used in a number of models to represent the low latitude electrodynamics. Measurements of the meridional drifts near the magnetic equator from the ROCSAT satellite (Fejer et al. 2008) averaged for moderate solar activity conditions are shown for all longitudes and local times in Fig. 21. Note how the diurnal variations have a marked dependence on longitude, with the strongest enhancements after sunset occurring in the longitude sector of -120 to +60 degrees East at the equinox.

Because the mid- and low-latitude ionosphere is a "closed" system (i.e., the magnetic field lines that encompass this region are all closed), it may appear straightforward to develop a self-consistent electrodynamic model based on the wind driven E- and F-region dynamos, even when electric field mapping and variations of magnetic field declination with longitude are taken into account. However, the dynamo-driven drifts are not the whole story. Tidal effects (discussed next) propagating from below also have profound effects in the ionosphere, and account for the 4-cell pattern in longitude in the morning vertical drifts shown in Fig. 21. In addition, neutral forcing from high latitudes as well as penetration electric fields of magnetospheric origin at all latitudes due to strong magnetic activity will also affect the global drift patterns. Finally, upwards propagating electric field contributions from tropospheric storms are also believed to contribute to the drifts at any given time, particularly at night when the plasma density is greatly reduced and the thunderstorm-generated electric fields couple more efficiently to the ionosphere.

\subsection{Effects of Tides on the Plasma Density and Electric Fields}

A very important contribution to the low and mid-latitude upper atmosphere is neutral forcing from below. The strongest and most regular forcing is due to solar thermal tides which exist over a wide range of harmonics related to the earth's 24 hour rotation period. Given the strong effect of neutral winds on the low and mid-latitude electrodynamics, it is not surprising that tidal forcing might have profound effects on the near-earth plasma. The significance of these tidal effects and the fact that they are well-organized, for example with longitude, have received renewed interest in the space physics community as new data sets have recently become available.

Solar tidal effects have been observed in the ionosphere in both the electrodynamics and the plasma density. The actual mechanisms by which the tidal signatures are manifest are still being debated. Apart from migrating tides which are responsible for creating day-night variations in the ionosphere, the most distinct tidal signature is attributed to the diurnal eastward wavenumber-3 (DE3) non-migrating tide which produces very clear longitude effects 
Fig. 21 Average vertical drift velocities recorded by the ion drift meter on the ROCSAT satellite, as a function of local time, longitude, and season, for quiet times during moderate solar flux conditions (Fejer et al. 2008)
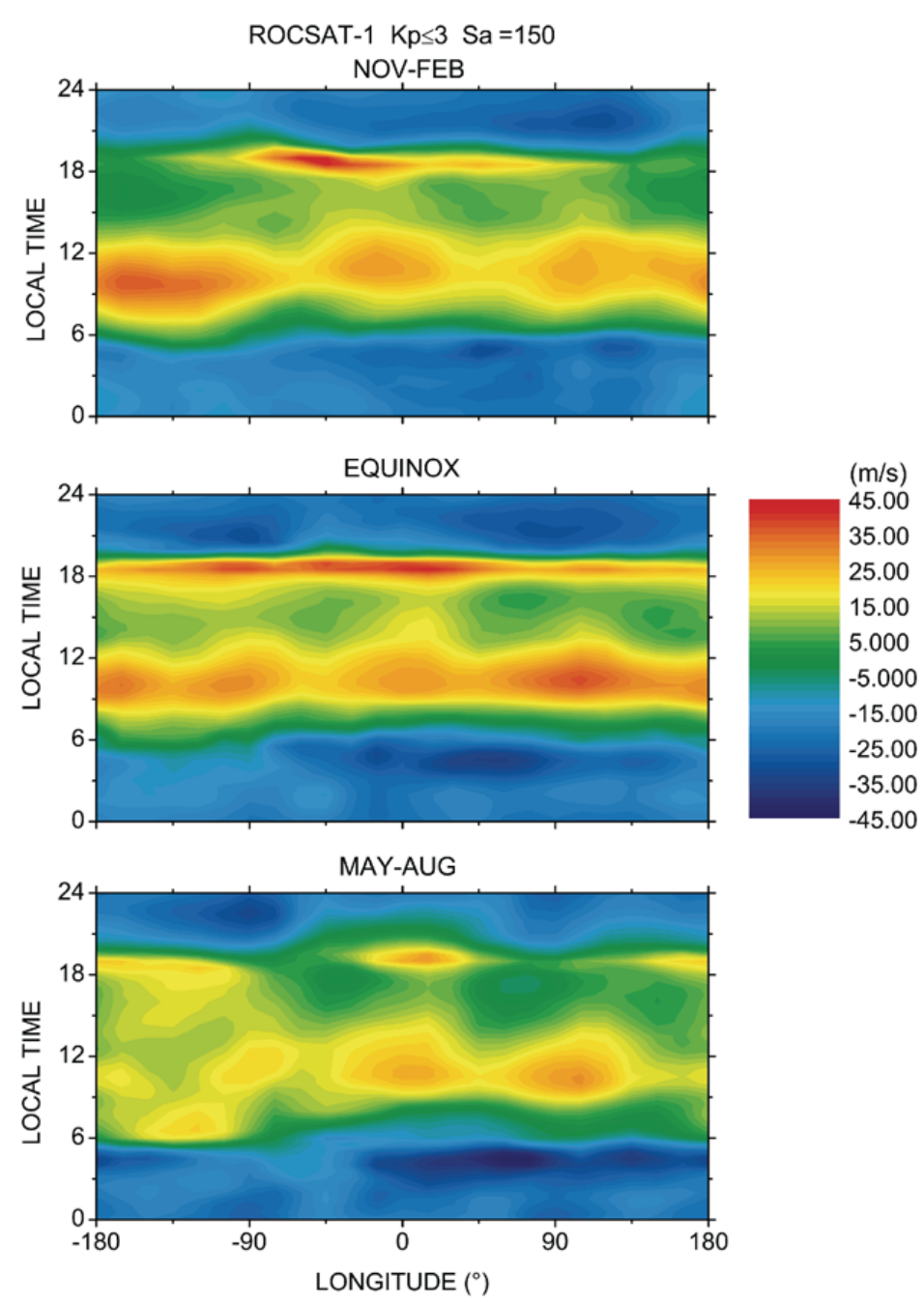

in the upper atmosphere. An example of how this tidal structure effects the low latitude, E-region electric fields is shown by England et al. (2006), who compared the equatorial electrojet strength as measured by the CHAMP, SAC-C, and Oersted satellite magnetometers and demonstrated that they each display the same 4-peak longitudinal structures. An example of enhancements in the total electron content at evenly-spaced longitudes at low latitudes for the 12-14 LT sector is shown in Fig. 22 using observations from the COSMIC satellites. (Figure provided courtesy of C. Lin. See Lin et al. 2007, for details.) These authors argued that the tides influence the F-region plasma by affecting the dynamo electric fields which subsequently modulate the vertical plasma transport. To demonstrate this, these authors used the same COSMIC data to create cross section plots of the equatorial ionization anomaly for a peak and a valley period to show that the EIA extended to higher altitudes and higher latitudes in periods of peak tidal forcing, a behavior which can be clearly linked to larger driving electric fields. That the equatorial electric fields show this same wave- 4 tidal signature is also evident in the 4-peak longitude variations of the vertical plasma drifts evident in the morning (9-11 LT) period in Fig. 21. A review of the effects of the non-migrating tides on the near-earth plasma is provided by England (2011, this issue).

Airglow enhancements in the crests of the equatorial anomaly peaks at 20 LT provides another representation of this non-migrating D3 tide phenomenon, as shown in Fig. 23 (Immel et al. 2006). This figure shows a reconstruction of 30 days of observations gathered with the FUV imager on the IMAGE satellite. The southern "band" is introduced as a mirror 


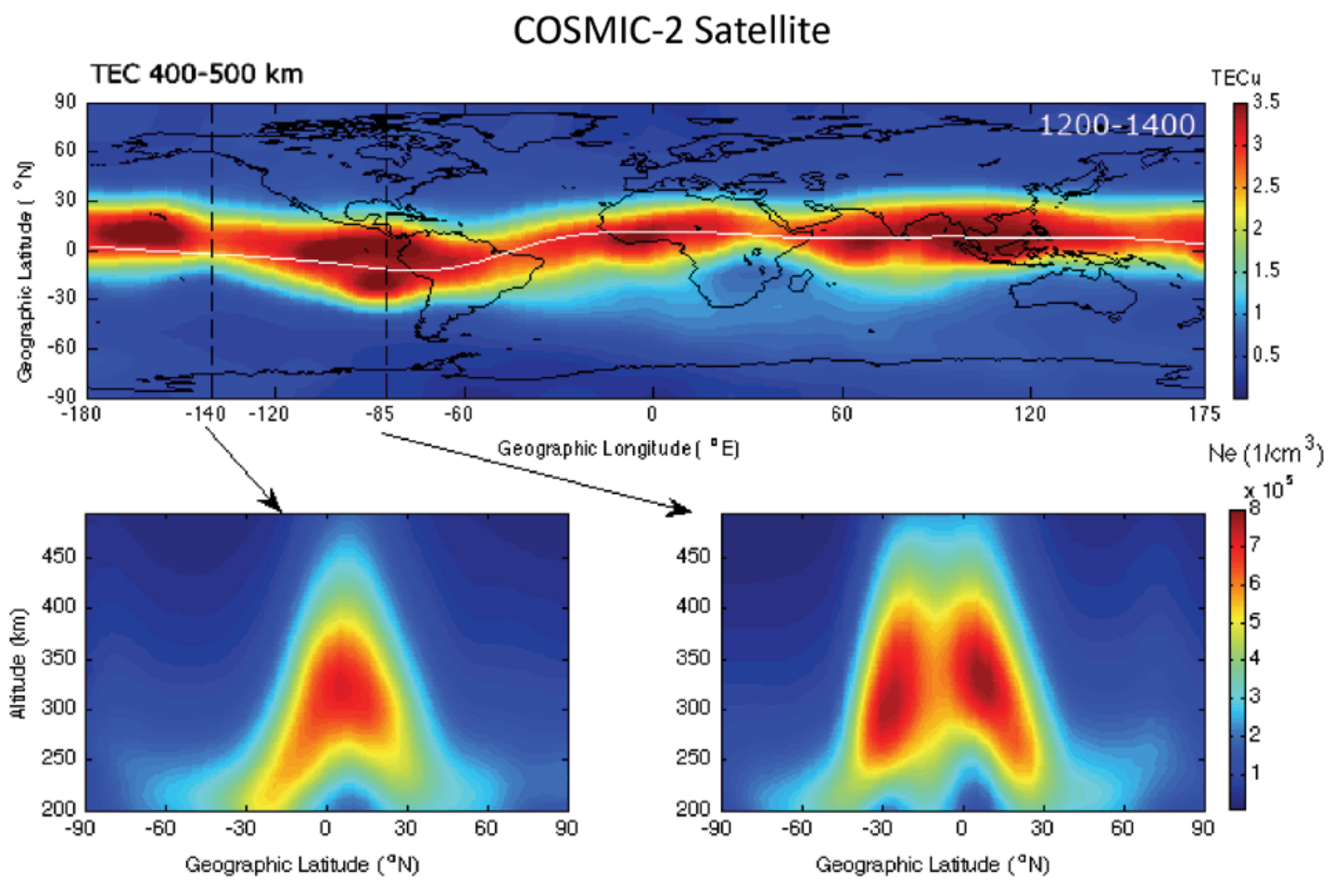

Fig. 22 Average TEC observations recorded by sensors on the COSMIC-2 satellites for the 1200-1400 LT sector as a function of latitude and longitude (upper panel). The lower panels show cross sections versus altitude and latitude corresponding to a minimum (left) and peak (right) TEC region, illustrating how the equatorial anomaly responded to different electric fields in each case (figure courtesy C. Lin. See also Lin et al. 2007)

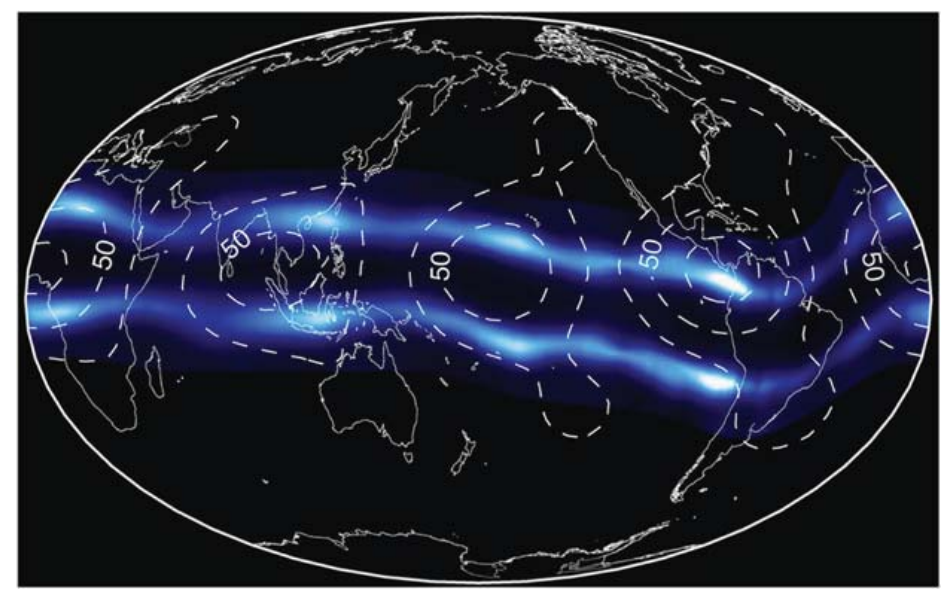

Fig. 23 Reconstruction of nighttime ionospheric emissions for March 20-April 20, 2002, as measured with the IMAGE satellite (Immel et al. 2006), which shows the average location and brightness of the equatorial anomaly. (Because of poor sampling of the southern hemisphere, its emissions are represented by a mirror image of the northern anomaly.) The image represents data at 2000 LT and includes an overlay of white dashed contours which represent model temperatures at $115 \mathrm{~km}$

image of the upper, northern band. The white dashed contours shown in this presentation represent model temperature variations due to the upward propagating tidal oscillations, which correlate nicely with the observed peaks in the equatorial anomaly. 
Fig. 24 Backscatter radar power at $50 \mathrm{MHz}$ as recorded on the night of March 6, 1983. Darker shades correspond to more power, which is depicted on a logarithmic scale (LaBelle 1985; Kelley et al. 1986)

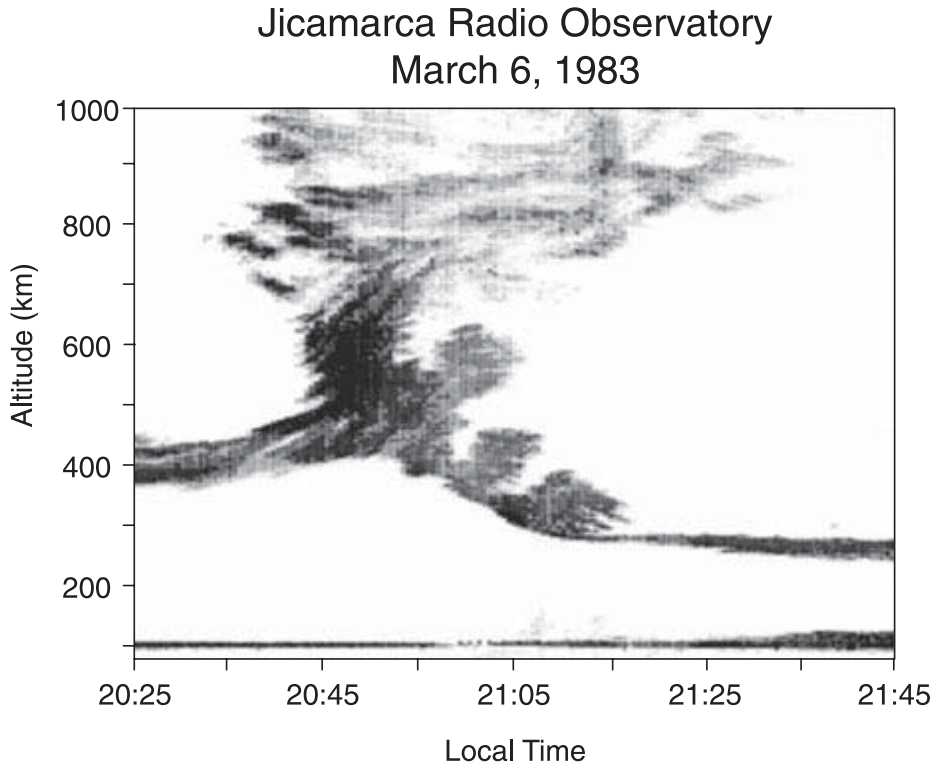

\subsection{Large Scale Plasma Depletions at Low Latitudes (“Spread-F”)}

At low latitudes at night, major distortions of the near-earth, ionospheric plasma may occur, often of planetary-scale proportions. This phenomenon may be characterized by a series of depleted magnetic flux tubes or wedges that stretch between magnetic conjugate locations in the northern and southern hemispheres. Sometime referred to as "bubbles", these elongated plasma depletions typically have internal, eastward electric fields of 5-20 mV/m (although sometimes much greater) and hence undergo significant upward $\mathbf{E} \times \mathbf{B}$ motions at speeds much greater than those of the background, ambient plasma (e.g., Aggson et al. 1992). The seat of the depleted flux tubes is well established to be the base or lower ledge of the nighttime F-region plasma (e.g., near 250 or $300 \mathrm{~km}$ ). When fully developed, however, these depletions can rise to altitudes of over $1000 \mathrm{~km}$ and extend significantly in latitude. In some cases, primarily off the magnetic equator, narrow plasma enhancements, instead of depletions, are observed (e.g., Le et al. 2003; Klenzing et al. 2011).

A key feature of the equatorial plasma depletions is that they are themselves unstable, and readily display strong electrostatic plasma irregularities with scale sizes that range from over $100 \mathrm{~km}$ to less than one meter. Certain scales contribute to Fresnel and Bragg scatter at high frequencies and cause radio scintillations as well as radar and communication outages (e.g., see de La Beaujardière 2004, and references therein). In fact, this general phenomenon was first identified by its associated irregularities, which create a spread in the F-region scatter on ionosonde traces and is generally referred to as "spread-F" in the literature. Recent reviews of large-scale plasma depletions/spread-F may be found in Woodman (2009), Kelley et al. (2011), and Makela and Otsuka (2011, this issue).

An example of equatorial spread-F is shown in the Jicamarca backscatter radar map for 3-m irregularities in Fig. 24 (Kelley et al. 1986; LaBelle 1985). Such radar "range-timeintensity" plots are similar to observations gathered with a slit camera: the radar beam has a fixed, one-dimensional (vertical) orientation through which the two-dimensional, dynamical ionospheric structures are observed. Thus, the reader must use caution in interpreting the image as a two-dimensional map, particularly because the features are not stationary, but evolve in space and time. With that precaution, we can nevertheless draw some important 
Fig. 25 Spatial relationship of the backscatter power measured during equatorial spread-F by the Altair radar at Kwajalein Atoll (lower panel) with the density measurement by the AE-E satellite on August 13, 1978 (after Tsunoda et al. 1982). The satellite trajectory is shown in red
AE Satellite - Plasma Density Measurements

Altair Radar (Kwajalein) - Backscatter Echoes 13 August 1978

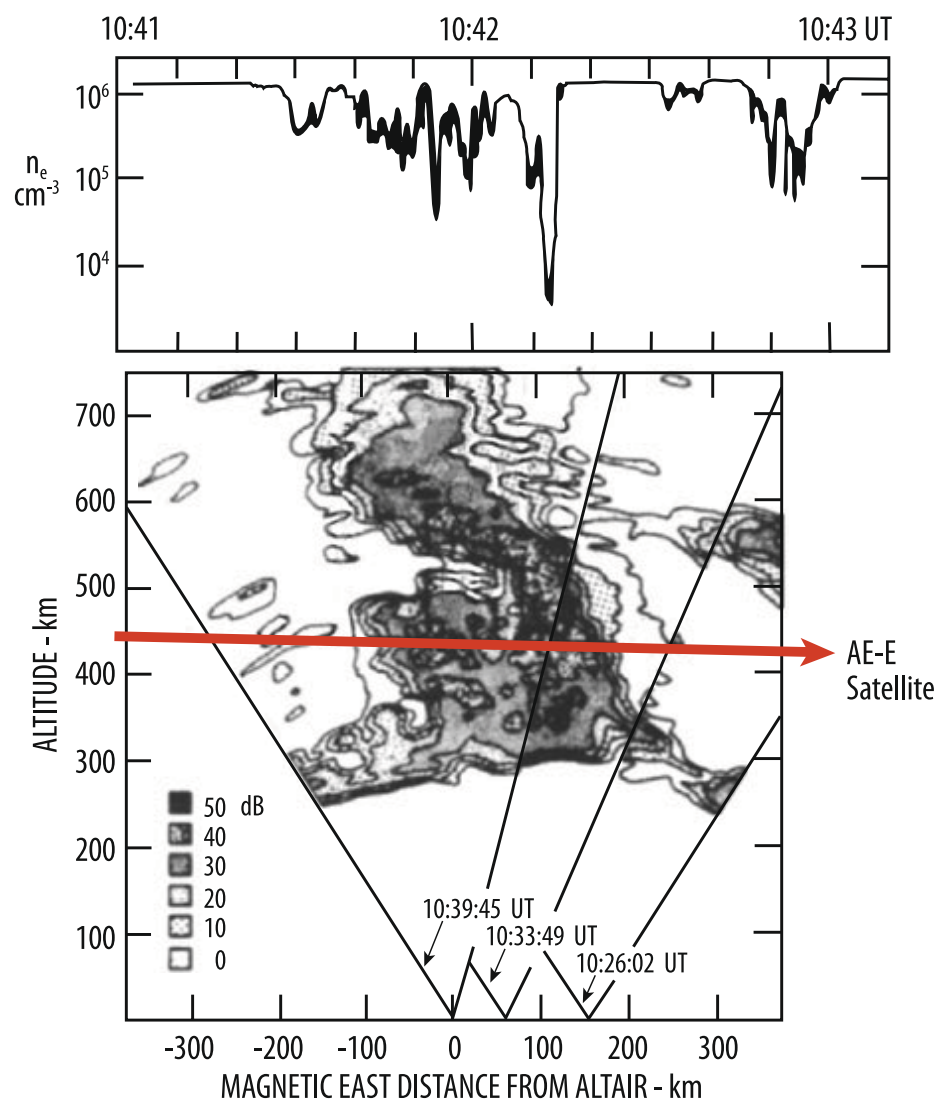

conclusions regarding large-scale ionospheric features and dynamics from this presentation. For example, the strong radar backscatter near $400 \mathrm{~km}$ at 20:25 UT almost certainly corresponds to the F-region ledge which then rises near 20:45 UT, presumably due to the pre-reversal enhancement of the eastward electric field, which causes the F-region to rise after sunset at low latitudes. The altitude of the ledge then decreases and settles at a range of about $300 \mathrm{~km}$ during the latter portion of the event shown (i.e., near 21:35 UT). This strong scatter that traces out the F-region ledge is known as "bottomside" spread-F.

The salient feature of Fig. 24 is the spread-F "eruption" shown in the center of the panel near 20:45 UT and extends to much higher altitudes, forming so-called "plumes". In fact, in this example, there appear to be 3 or perhaps 4 evenly spaced plumes that emerge from the center and backside of the F-region ledge (between 20:45 UT and 21:15 UT) whose irregularities constitute "topside spread-F". Furthermore, note that above about $700 \mathrm{~km}$, there are very high altitude structures that display a more diffuse character. Such scatter is believed related to smaller scale instabilities that are driven at high altitudes where the collision frequencies are negligible. Finally, we note that the narrow, darkened traces near $100 \mathrm{~km}$ correspond to nighttime electrojet irregularities, for which the decrease in intensity near 21:00 likely corresponds to the change in the direction of the ambient zonal DC electric field, as shown for other examples in Fejer et al. (1975).

We now consider another radar backscatter example, in this case from the steerable Altair radar at Kwajalein, which the AE-E satellite traversed with a plasma density probe, as shown in Fig. 25 (Tsunoda et al. 1982). In this case, as shown in the upper panel, the probe clearly 


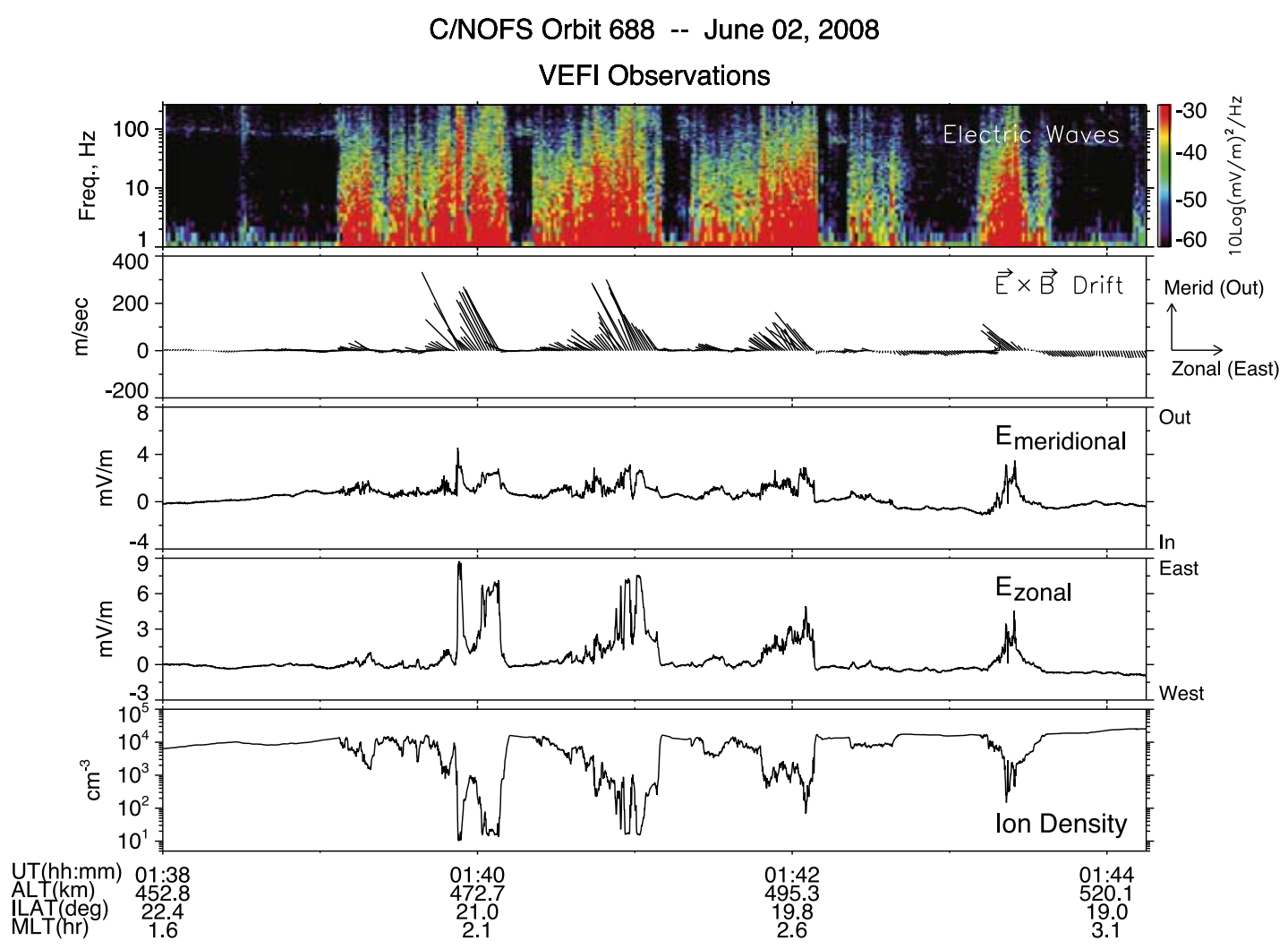

Fig. 26 Electric field and plasma density measurements recorded by the C/NOFS satellite during strong spread-F density depletions, as shown in the bottom panel. The two panels above the density show the DC electric field components and the panel above that shows the corresponding $\mathbf{E} \times \mathbf{B}$ velocity. Notice how the zonal component of the electric field, which corresponds to the outward plasma drift component, is anti-correlated with the log of the plasma density. The uppermost panel shows ELF electric field spectra that reveal strong irregularities coincident with the density depletions

observes profound plasma density depletions, decreasing by over two orders of magnitude, which correspond precisely to the regions of strong radar backscatter, as shown in the lower panel. This example and many others have demonstrated conclusively that strong backscatter echoes originate in regions of well-defined, abrupt plasma depletions.

An example of C/NOFS satellite probe observations of plasma density depletions and associated electric fields within the depletions is shown Fig. 26. The data show a series of depletions that extend to very low plasma densities and which have associated upwards and westward $\mathbf{E} \times \mathbf{B}$ drifts, as measured by the vector electric field detector on that satellite. Note how the zonal electric field and the log plasma density are anti-correlated, even at small scales, indicative of a quasi-linear relation between these quantities, as shown by Aggson et al. (1992) for similar observations using data from the San Marco satellite. The C/NOFS data in the uppermost panel of Fig. 26 show electric field irregularities with the associated depletions. These are the irregularities that disrupt radiowaves, as discussed above.

Although the definitive source of the onset and triggering of such low latitude plasma depletions is still debated, the rising, depleted flux tubes are believed a consequence of an interchange or Rayleigh-Taylor instability (e.g., Woodman and La Hoz 1976). In this case, the plasma density at low altitudes $(<250 \mathrm{~km})$ is greatly reduced at night so that there exists a "heavy" fluid (i.e., the F-region ionosphere) suspended over a "lighter" fluid. The instability is particularly susceptible near the magnetic equator because the magnetic field lines in the F-region are nearly horizontal and essentially sustain the higher density plasma at higher 
Jicamarca Combined Incoherent and Coherent Scatter 24 February 1996

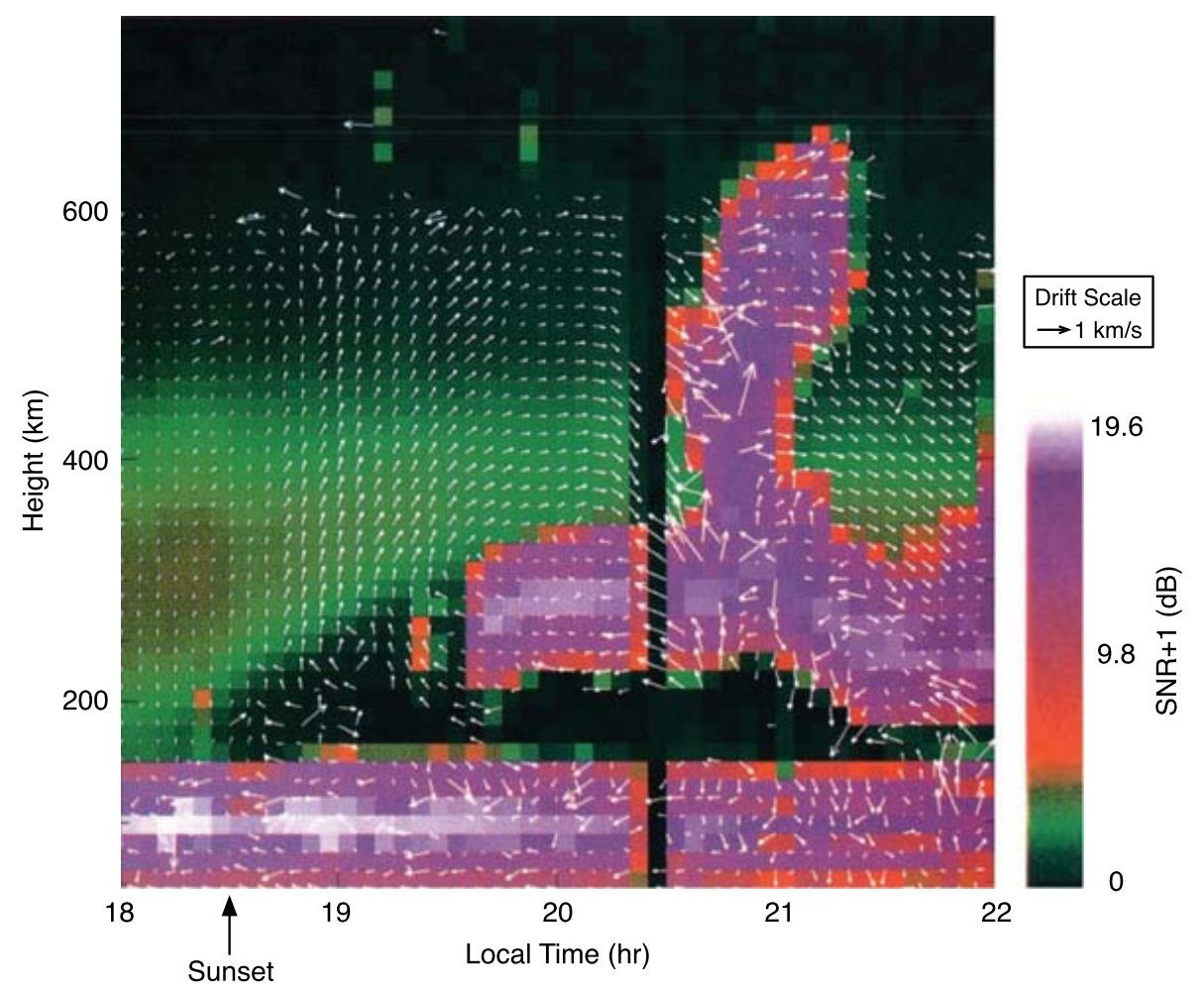

Kudeki and Bhattacharyya [1999]

Fig. $27 \mathbf{E} \times \mathbf{B}$ drifts (white arrows) and Jicamarca backscattered power map for the early evening hours of February 24, 1996 (Kudeki and Bhattacharyya 1999). The scattered power corresponds to incoherent scatter (brown and green shades), and shows how the lower edge of the ionosphere rises after sunset, which occurred at 18:32 LT. The stronger scatter (red and purple shades) correspond to coherent scatter of the spread-F and E-region irregularities. Notice the vortex pattern in the $\mathbf{E} \times \mathbf{B}$ arrows prior to the onset of the high altitude plume that begins near 20:30 LT

altitudes. Small perturbations on the F-region ledge would produce an eastward electric field which grows in concert with an associated density depletion, starting the instability process. Another factor believed important for irregularity development is that at sunset, large pre-reversal, zonal electric fields cause the F-region to rise suddenly to altitudes more susceptible to shorter scale instability growth (e.g., Kelley 2009).

Despite the apparent success of the Rayleigh-Taylor instability explanation for the evolution of low latitude plasma depletions, it is widely acknowledged that this instability is too slow to produce the observed prompt depletions after sunset and an additional triggering mechanism is needed to explain their rapid appearance after sunset. Furthermore, the presence of a heavy fluid over a light fluid at the magnetic equator occurs every night, so some additional instigator is required beyond statistical fluctuations. Recent theoretical analysis has shown that spread-F depletions may be triggered whenever eastward neutral winds propagate at different speeds than that of the ambient plasma, as discussed by Kudeki et al. (2007). These authors show how a vortex in the ambient plasma at sunset (in the plane perpendicular to the magnetic field) contributes to the fact that the plasma and neutral gases subsequently move at different velocities. An example of the vortex motion in the plasma drift detected by the Jicamarca radar is shown in Fig. 27 (Kudeki and Bhattacharyya 1999). Shear instabilities also have been suggested to create the large-scale undulations that ulti- 


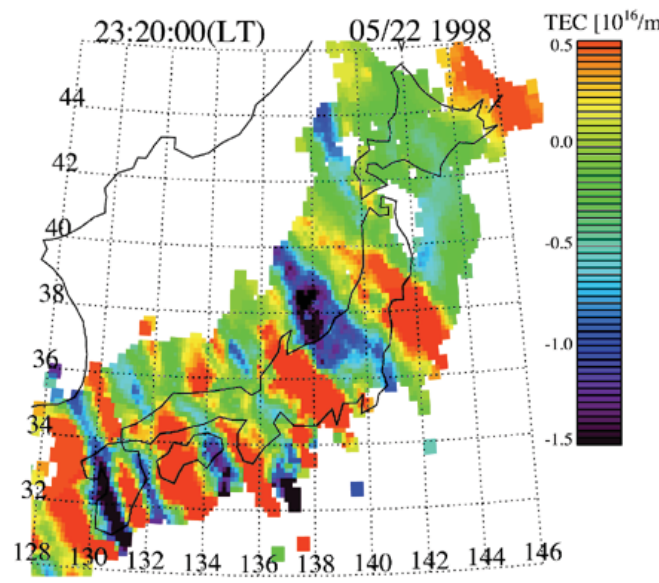

(a)

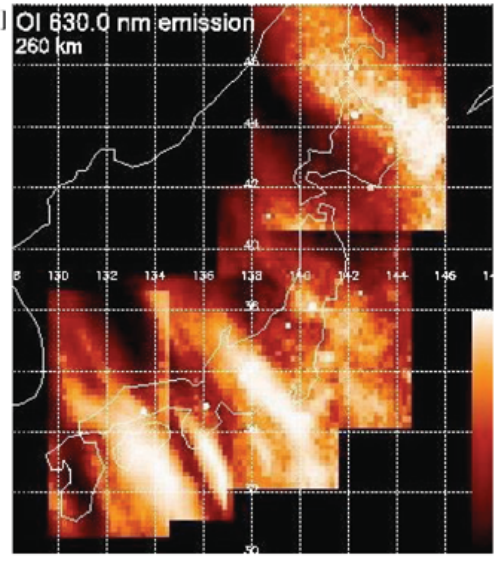

(b)

Fig. 28 Simultaneous distributions of total electron content showing gravity waves/TIDS at 23:20 LT on May 22, 1998 using GPS data (a) and $630 \mathrm{~nm}$ airglow data (b) (Saito et al. 2001)

mately seed the Rayleigh-Taylor growth of spread-F (e.g., Hysell and Kudeki 2004). This shear in the zonal plasma drift at the base of the F-region was observed in altitude profiles of the vertical electric field measured on sounding rockets flown from Kwajalein Atoll, as reported by Hysell et al. (2006).

\subsection{Acoustic Gravity Waves and Traveling Ionospheric Disturbances}

Acoustic gravity waves have been known for some time to affect the ionosphere and, at some level, such oscillations are expected to be ubiquitous in the ionosphere. In general, apart from singular events driven by localized phenomena such as earthquakes, two sources of gravity waves in the mid and low-latitude ionosphere are customarily studied: (1) upwardspropagating gravity waves that originate in the lower atmosphere, such as those generated by thunderstorms and other tropospheric energy sources (e.g., Walterscheid 2001), and (2) horizontally-propagating gravity waves generated at high latitudes, for example by auroral processes, that subsequently propagate equatorward (e.g., Crowley 1991). The neutral motions associated with the gravity waves set the ionosphere in motion where they are observed as traveling ionospheric disturbances, or TIDs. In a situation similar to that of tidal forcing, recent advances in instrumentation and observations have enabled a more in-depth analysis of the role on gravity waves/TIDs in near-earth region of geospace.

Traveling ionospheric disturbances are frequently observed in airglow, radar, and GPS measurements. Recently, arrays of GPS receivers have shown the organization of gravity waves/TIDs traveling equatorward from the poles. Figure 28 shows TIDs evident in simultaneous GPS and airglow data over Japan, clearly revealing the large scale density variations associated with such events (Saito et al. 2001). TIDs are generally grouped into large scales $(>1000 \mathrm{~km})$ and mesoscales, (scalelengths between 300 and $1000 \mathrm{~km}$ ), the latter being termed MSTIDs in the literature. The fact that such waves include electric fields which can easily map along the magnetic field lines explain conjugate MSTID measurements observed by Shiokawa et al. (2004) and Otsuka et al. (2004). The coupling processes between the E and $\mathrm{F}$ region ionosphere are now being modeled (e.g., Yokoyama et al. 2009) and underscore the importance of gravity wave sources in understanding the mid-latitude ionospheric structure. 
Fig. 29 The shear in the neutral wind creates a layer of enhanced ionization (after Hines 1964)

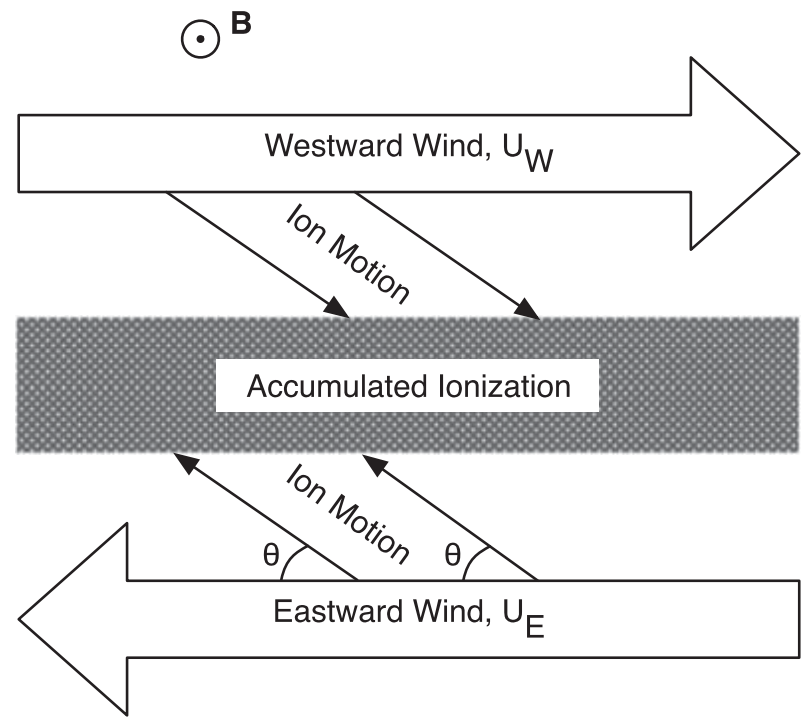

Using measurements from the Dynamics Explorer-2 satellite gathered near the end of its mission between 250-300 km, Earle et al. (2008) reported gravity wave measurements at mid-latitudes, comparing simultaneous waveform measurements of the neutral density, plasma density, ion velocity, and neutral velocity perturbations. They showed plasma density variations $(\Delta \mathrm{N} / \mathrm{N})$ of approximately $30 \%$ with scale lengths along the satellite orbit of over $100 \mathrm{~km}$, and argued that because the neutral density fluctuations are observed to lag their neutral vertical velocity by 90 degrees (phase quadrature), the perturbations are gravity waves as predicted by Hines (1964). They also showed that the observations agree with gravity wave dissipation models of Vadas and Fritts (2005) and Vadas (2007). These authors argued that such measurements are more common at the lower altitudes, below where most ionospheric satellite probes generally gather their measurements.

\subsection{Distinct Metallic Ion Layers in the Lower Ionosphere (“Sporadic-E” Layers)}

In Sect. 2, we showed that the main ions in the lower ionosphere $(<150 \mathrm{~km})$ were $\mathrm{NO}^{+}$ and $\mathrm{O}_{2}^{+}$. Missing from this description of the near-earth plasma environment is the fact that this region is often characterized by distinct, heavy ion layers that are common in both the daytime and nighttime ionosphere and may be somewhat more prevalent at mid-latitudes. Such layers are often termed "sporadic-E" layers, since they occur in the lower E-region and may abruptly influence radiowave applications as they drift within the field-of-view of beams of HF radiowave communication antenna. In fact, the layers are actually much more ubiquitous than this name implies. We nevertheless retain this nomenclature for the discussion here.

Sporadic-E layers are dramatic examples of neutral-plasma coupling that routinely occur in the earth's lower ionosphere/thermosphere system. Typically forming in the altitude region of 90-130 km, it is fairly well established that such layers are set up through a combination of neutral wind shear and the effects of long-lasting metallic ions resulting from meteoric input (e.g., Whitehead 1970; Mathews 1998; Haldoupis 2011, this issue). That sharp, distinct sporadic-E layers result from the occurrence of shears in the zonal neutral wind, as advanced by Hines (1964), Whitehead (1970), and others, is shown conceptually in Fig. 29 (after Hines 1964). Such layers may then be modulated by upward propagating gravity waves and shear instabilities (see discussion below), possibly creating polarization electric fields within the crests and troughs of the modulated layer. 


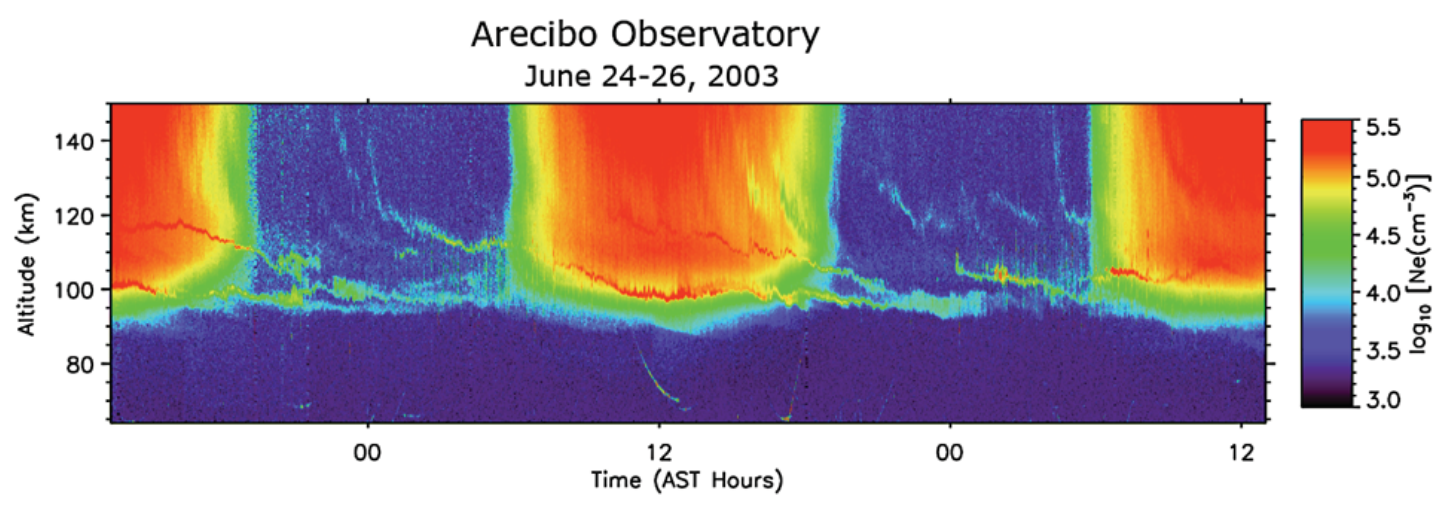

Fig. 30 Incoherent scatter measurements of the plasma density in the lower ionosphere for two consecutive days at the Arecibo Observatory in Puerto Rico. Note the sporadic-E layer continues unbroken between day and night

To illustrate the character of sporadic-E layers, altitude profiles of continuous plasma density observations from the Arecibo incoherent scatter radar are shown in Fig. 30 for two full days, in a format similar to that of Fig. 9, except the focus is now on the E-region. The narrow layers of enhanced ionization that are more distinct at night but are also present during the day are the sporadic-E layers. Of particular importance is the fact that these layers transition from day to night without any apparent break, and they descend, in general, with local time, although occasionally the layers "jump" to slightly higher altitudes.

In addition to research carried out with radars and ionosondes, sounding rocket experiments have provided considerable insight to the formation and character of sporadic-E layers. In particular, large amplitude neutral winds and neutral wind shears have been observed in vapor trails released along near vertical rocket trajectories and shown to exist at the same altitudes of simultaneously observed sporadic-E layers (see, for example, Larsen et al. 1998, and references therein). Those authors also showed calculations of the Richardson number based on the measured wind profile, demonstrating that the altitude range near the layer was highly unstable.

In another experiment, a sounding rocket was launched in conjunction with observations of sporadic-E layers recorded by the incoherent scatter radar at Arecibo, Puerto Rico. The top panel of Fig. 31 presents sporadic-E layers between 100 and $110 \mathrm{~km}$ in continuous plasma density profiles obtained with the Arecibo radar during the evening when a sounding rocket was launched from the north coast of Puerto Rico at 21:45 LT, as indicated by the arrow. The rocket payload included an ion mass spectrometer from which data in the lower panel of Fig. 31 clearly shows that the lower density layer was composed primarily of $\mathrm{Fe}^{+}$ and $\mathrm{Mg}^{+}$ions (Earle et al. 2000). These results are in agreement with previous mass spectrometer measurements of metallic ions associated with sporadic-E layers (see Grebowsky and Aikin 2002, and references therein). Earle et al. (2000) used the observed separation with altitude of the different metallic layers to estimate the zonal winds necessary to sustain the layer against diffusive effects.

The combined action of neutral wind shears, electric fields, and the sharp plasma density gradients intrinsic to the sporadic-E layer subsequently drive instabilities that include features that are detectable by radars on the ground. In fact, an important consequence of the wind shears is the fact that they can be unstable to the Kelvin-Helmholtz instability. Such a process has been shown by Larsen (2000) to account for the quasi-periodic (QP) radar echoes that have been regularly reported in backscatter radar data associated with sporadic-E layers (e.g., Yamamoto et al. 1991; Hysell et al. 2004). The effects of the Kelvin-Helmholtz 


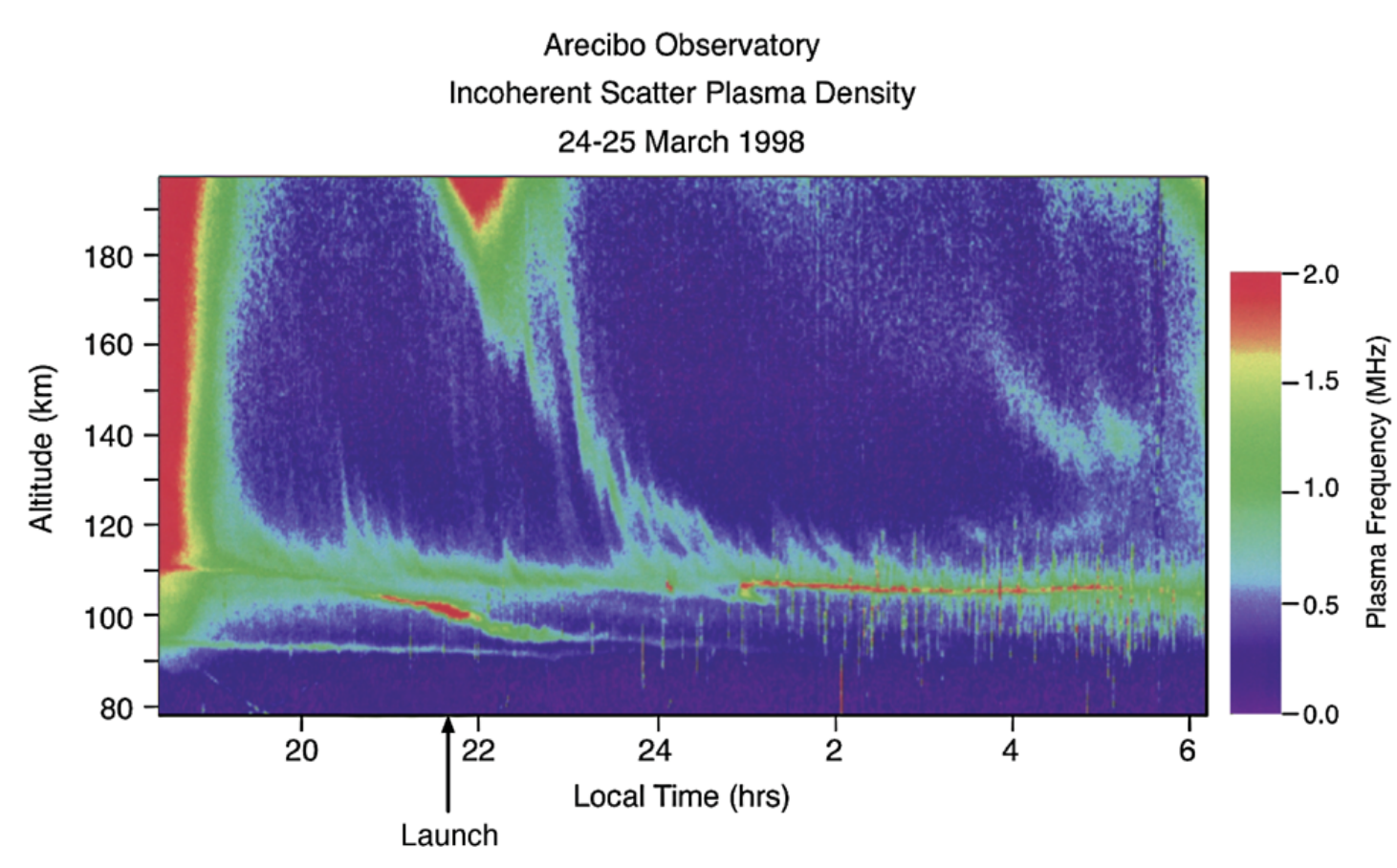

NASA 21.115 -- Tortuguero Bay, Puerto Rico

24 March 1998 at 01:45:21 U.T.

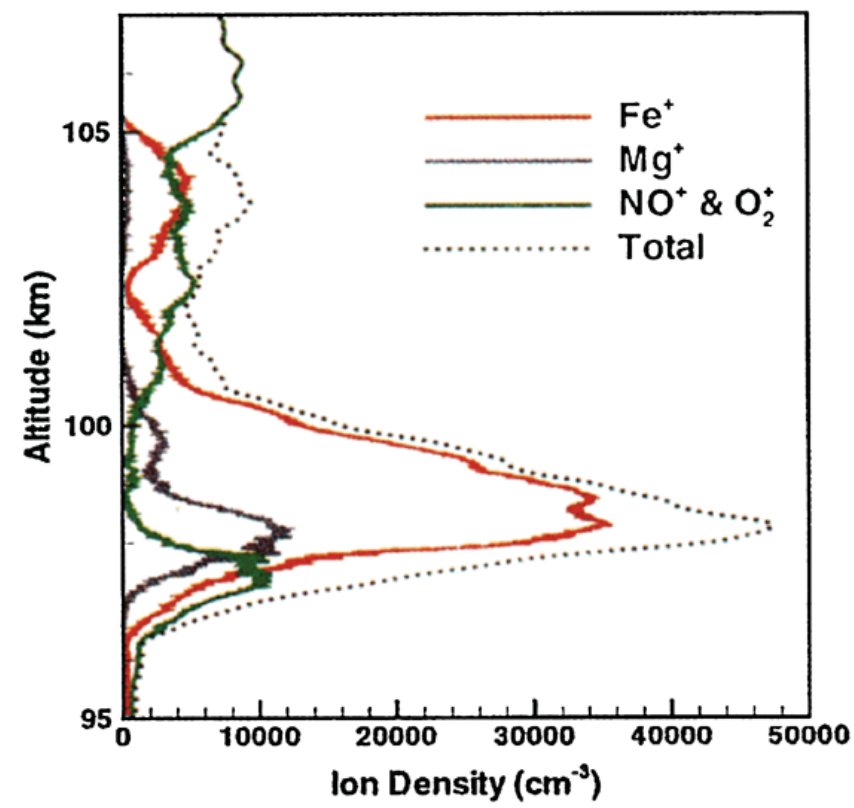

Fig. 31 Incoherent scatter measurements of the plasma density on the night of March 24-25, 1998 at the Arecibo Observatory in Puerto Rico (upper panel). A sounding rocket was launched at 01:45:21 UT (see arrow in top panel) which recorded the ion composition data shown in the lower panel (after Earle et al. 2000)

instability on a low altitude, nearly horizontal vapor trail released from a sounding rocket in the presence of sporadic-E and QP-echoes have been analyzed by Larsen et al. (2005). In this experiment, periodic whirls are clearly visible in the vapor emissions emitted along a horizontal trajectory in the E-region, suggesting that the Kelvin-Helmholtz instability was at work during this experiment. 


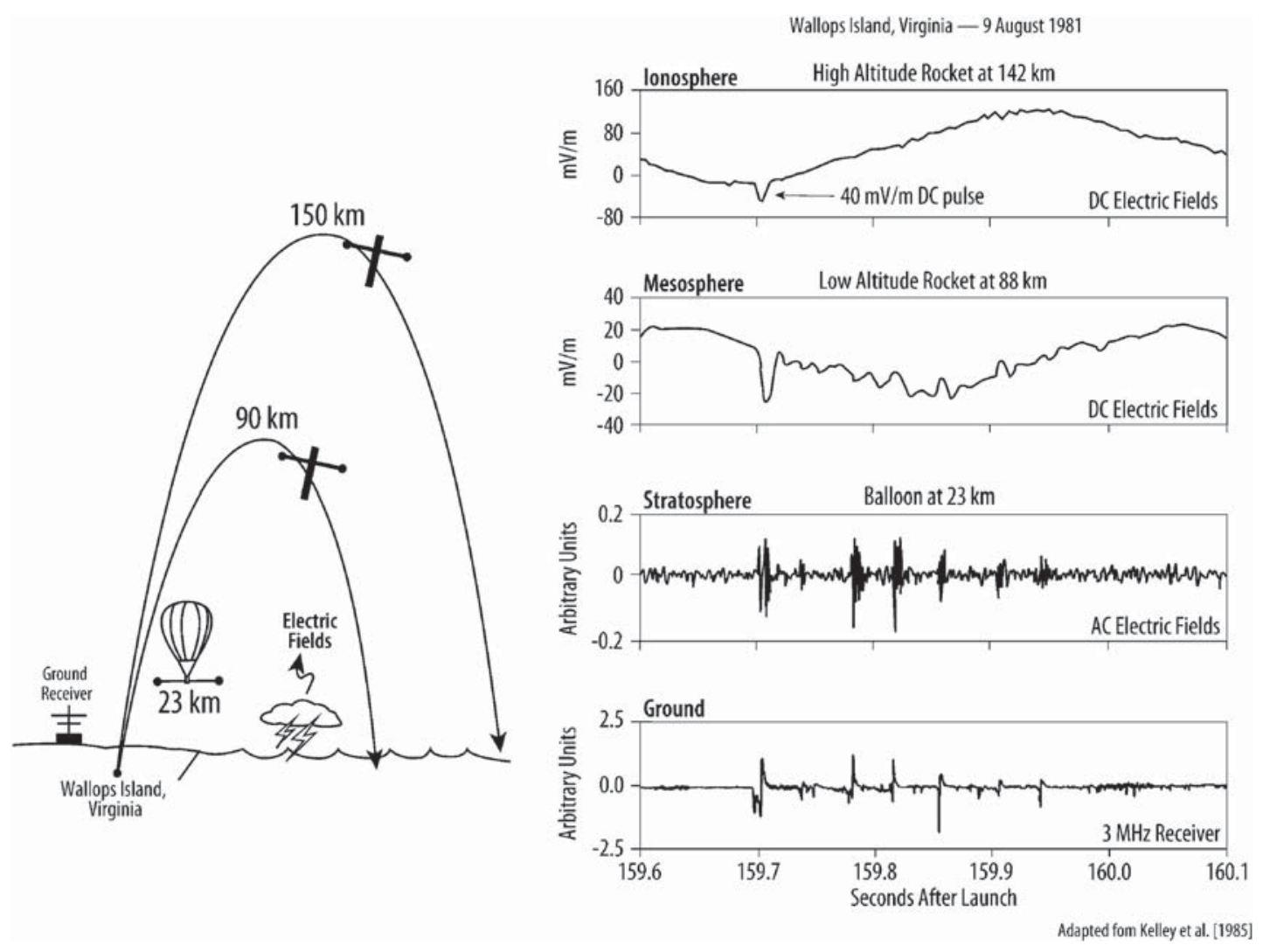

Fig. 32 Electric field measurements recorded over an active thunderstorm by two rockets, a balloon, and a ground receiver (after Kelley et al. 1985). The sketch on the left shows the experiment geometry. The panels on the right show data from each platform for one half-second period when one rocket was in the ionosphere and one in the mesosphere. Note how the DC electric field pulse is observed simultaneously in both rocket data sets and appears to penetrate upwards, into the ionosphere. The large amplitude, very low frequency sinusoidal variations in the upper two panels are due to the spin of the rockets

Finally, we mention another layer phenomenon commonly observed in the mid-latitude ionosphere below roughly $200 \mathrm{~km}$ - the so-called "intermediate" or "descending" layer (e.g., Earle et al. 1998; Mathews 1998). An example is shown in the Arecibo density profiles in the top panel of Fig. 31. A descending layer appears to evolve from a density bulge associated with the lower edge of the F-region near 22 LT, from which a low amplitude, but distinct, density enhancement transitions to lower altitudes during the next hour or so. The creation and dynamics of such layers are not fully understood.

\subsection{Electrical Contributions to the Upper Atmosphere Due to Tropospheric Storms}

The lower edge of the ionosphere has been recognized for some time as the conductive upper layer of the earth-ionosphere waveguide. As such, it provides an essential part of the global circuit driven by atmospheric electricity (e.g., Wilson 1920). The ability of low frequency (DC-ELF) electric fields associated with tropospheric storms to penetrate into the ionospheric plasma remains a topic of considerable discussion (see Simoes et al. 2011, this issue).

To investigate the extent of electrical energy deposition in the ionosphere, Kelley et al. (1985) reported observations gathered by a pair of sounding rockets launched simultaneously to apogees in the mesosphere and ionosphere over an active thunderstorm at a midlatitude location (Wallops Island, Virginia, USA). As shown in Fig. 32, the results clearly 
demonstrated that the DC electric field "pulse" associated with lightning spherics penetrated into the ionosphere. Besides requiring a revision of models of the transfer of electromagnetic energy across the atmosphere/plasma interface, these results demonstrated that significant energy is deposited in the ionosphere by lightning-generated electric fields. These researchers also reported a significant component of the DC electric field pulse was oriented parallel to the magnetic field direction in the lower ionosphere. Such an electric field would be expected to contribute to localized plasma heating, as discussed by those authors.

The effects of tropospheric storms on the ionosphere may include other consequences. Burke et al. (1992) reported Dynamics Explorer-2 observations of Alfvén waves near $350 \mathrm{~km}$ altitude over a hurricane that show a DC electric field pulse of more than $40 \mathrm{mV} / \mathrm{m}$ with an associated plasma density depletion and strong magnetic signature. These authors argued that this signature was due to Alfvén "wings" associated with an intense upwardspropagating DC electric field generated by the hurricane forces. Similarly, using data from the San Marco satellite, Farrell et al. (1994) reported large, localized DC electric field "structures" in the ionosphere over tropical storms, providing further evidence that DC-coupled electric fields associated with tropospheric storms propagate upwards and penetrate into the ionosphere. These and numerous other observations underscore how the ionosphere is significantly influenced by energy and momentum sources originating in the troposphere below. We now turn to the high latitude ionosphere and consider energy and momentum sources that originate from above, in the magnetosphere.

\section{The High Latitude Ionosphere}

The ionosphere at high latitudes differs significantly from that at low and mid latitudes. At high latitudes, the ionosphere exchanges energy and momentum with the solar wind/magnetosphere primarily via the highly inclined magnetic field lines that characterize the earth's polar regions. The magnetic field thus serves as a conduit for both charged particles and their associated field-aligned currents and DC electric fields, thus directly coupling the high latitude ionosphere and upper atmosphere with the magnetosphere. Although photoionization still contributes to the ambient plasma environment, an alternative, often dominant, source of ionization in the high latitude ionosphere is impact ionization of energetic particles with the neutral atmosphere, and the primary drivers of high latitude ionosphere dynamics are electric fields that map down from the magnetosphere. In contrast, the primary source of ionization for the low and mid-latitude ionosphere is photo-ionization from the sun and the primary drivers of plasma transport are neutral winds and small amplitude electric fields created by the neutral winds.

In this section, we introduce the visual aurora and the different populations of precipitating electrons that create the aurora. We discuss the creation of thermal plasma by auroral precipitation and compare this with photoionization. We then review the dynamics and currents of the high latitude ionosphere associated with magnetospheric processes. This is followed by a discussion of the many fundamental processes in which the high latitude plasma environment interacts with the upper atmosphere. We conclude with some brief remarks regarding auroral arc electrodynamics.

\subsection{The Visual Aurora and Energetic, Precipitating Auroral Particles}

Overview of the Visual Aurora. Optical emissions associated with the aurora result from interactions of energetic, precipitating particles with the upper neutral atmosphere. Typically 


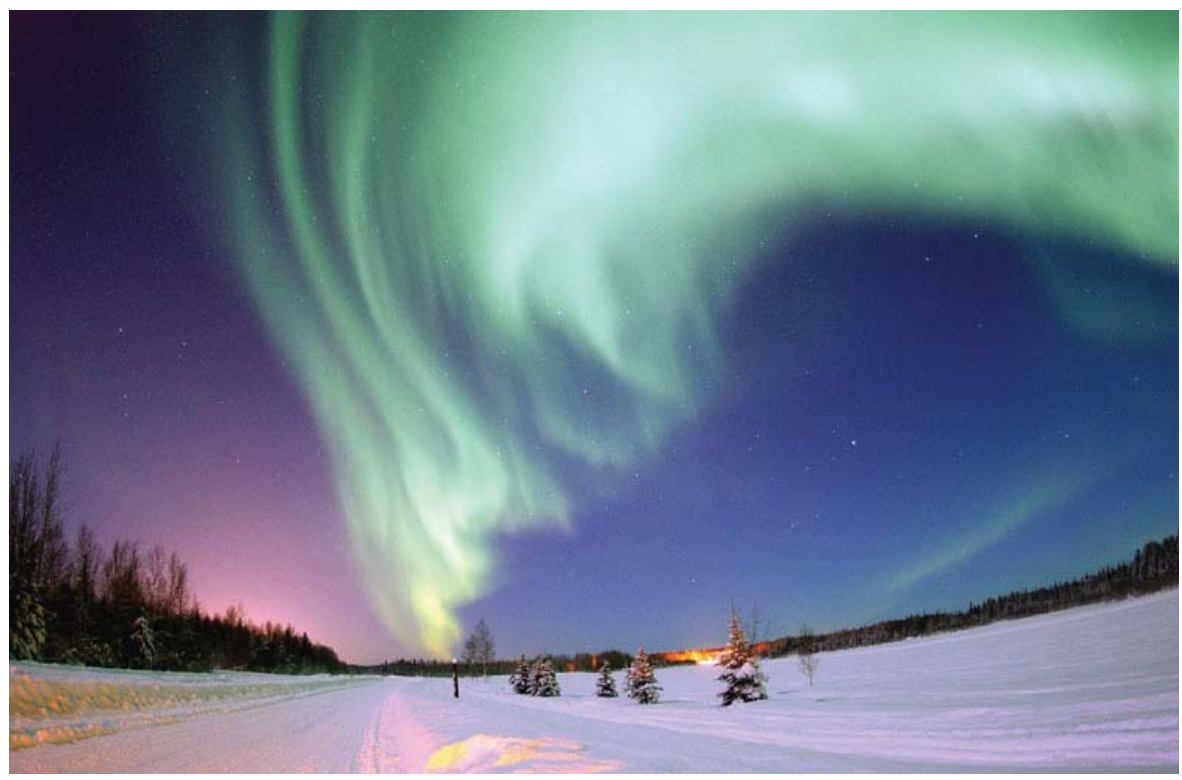

Fig. 33 Photograph of the aurora taken in January, 2005 at Bear Lake, Alaska. Credit: Joshua Strang, USAF

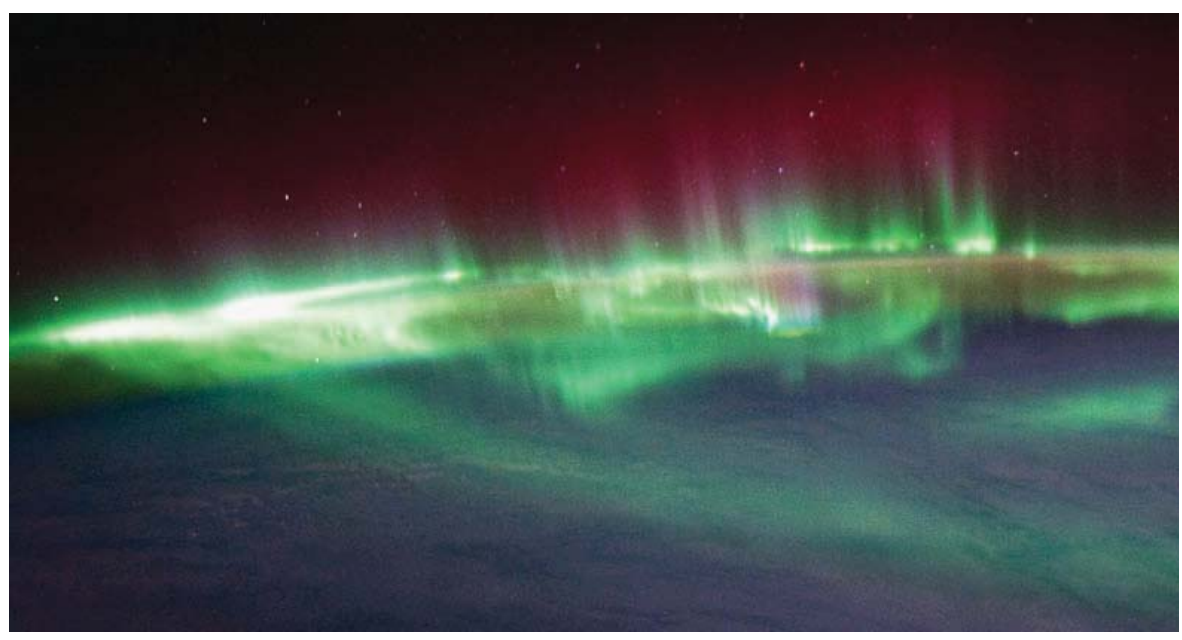

Fig. 34 View of the aurora photographed from the International Space Station on March 4, 2012, 17:20:33 UT on a pass over the South Indian Ocean. Figure courtesy NASA

produced within the altitude region of 80-250 km, displays of discrete auroral arcs are perhaps the most striking, visible manifestations of the interactions of space plasma-in this case, precipitating energetic electrons - with atmospheric, neutral gases. Figure 33 is an auroral display photographed from the ground that shows sheets of auroral emissions, which are modulated by large amplitude spatial undulations that have typical durations of seconds to minutes. Another view of such auroral "curtains" is shown in Fig. 34. In this case, the side of the aurora has been photographed from the NASA International Space Station, at an altitude near $400 \mathrm{~km}$. Note that the auroral emissions are characterized by nearly vertical striations that correspond to the ambient magnetic field lines along which the precipitating particles are guided. The green glow at the base of the aurora is the most common auroral hue $(\lambda=557.7 \mathrm{~nm})$ and is due to interactions of the more energetic electrons with oxygen at the lower altitudes $(100-130 \mathrm{~km})$ whereas the pink-red glow $(\lambda=630.0 \mathrm{~nm})$ in the upper 
portion of the auroral display is from interactions of less energetic electrons with oxygen at higher altitudes (130-250 km). Other, less common, colors of auroral displays include bright red and blue, which correspond to emission lines associated with the interaction of precipitating energetic particles with other gases such as $\mathrm{N}_{2}$.

An image of the aurora gathered by the DMSP satellite above the southern polar region is shown in Fig. 35 and depicts discrete arcs, diffuse aurora, and auroral forms filled with turbulent-like structure. This image demonstrates that, in the aggregate, auroral emissions and the processes that create them are essentially global in their extent, expanding across the polar region with a variety of patterns in which shorter scale structures are embedded. The ensemble often resembles an auroral oval (sometimes referred to as an auroral belt), as is illustrated in the images with the wider field of view shown in Fig. 36. This time sequence of images, gathered every 12 minutes by the imager on the Dynamics Explorer-1 satellite, illustrates how dynamic the large scale aurora may be, in this case brightening and expanding poleward on the nightside during a magnetospheric substorm. (See Frank and Craven 1988 for a discussion of the auroral images from this instrument.)

The source of the auroral optical emissions shown in the preceding figures is energetic electrons with typical energies of 100's of eV to 10's of keV that precipitate into the high latitude neutral gases where they impact and are "braked". Here, such energetic electrons interact with the neutral atmosphere in two basic ways: excitation and ionization. Excitation occurs when an energetic electron strikes a neutral atom or molecule and excites the neutral to a higher energy state. When the neutral relaxes to a lower energy state, a photon is released, creating the visible aurora. Ionization of the ambient neutral gas occurs when the incoming energetic electrons impact the neutral atoms or molecules and liberate electrons to create ions, thermal electrons, and more energetic secondary electrons. In the remainder of this sub-section, we discuss the different types of aurora and their relative distributions in the high latitude ionosphere. We then discuss the ionization associated with the precipitating particles in the following sub-section.

The aurora may be categorized into three primary types that depend on their respective acceleration or precipitation processes and on the characteristics of their associated precipitating energetic particles: (1) discrete, (2) oscillating or Alfvénic, and (3) diffuse. Each are introduced below, followed by comparisons of their relative number flux and energy flux contributions to the high latitude ionosphere. Although we mention other auroral features as they pertain to the near-earth plasma environment, we do not attempt to review all of the aurora-related processes here. Readers are directed to many reviews of auroral phenomena and related physical processes, including those by Rees (1989), McFadden et al. (1999), Lummerzheim (2007), and Newell et al. (2009). In particular, the ISSI monograph entitled, Auroral Plasma Physics, edited by Paschmann et al. (2003), contains a thorough compendium of current understanding of the physical processes associated with the aurora.

Discrete Aurora. The visual aurora shown in Figs. 33, 34, 35, 36 are, in general, associated with discrete auroral arcs. In situ measurements by probes on sounding rockets and satellites have shown that the precipitating energetic electrons associated with discrete auroral arcs have narrow, nearly monoenergetic spectra (e.g., McIlwain 1960; Evans 1968). These electron spectra are typically organized in "inverted-V"-shaped energy functions that correspond to spatial variations across the discrete auroral arc (Frank and Ackerson 1971). (The inverted-V's tend to resemble inverted-U's where the energy is displayed as a logarithm.) The peak energies of such energetic electrons typically range from 100 's of eV to a few tens of keV. In Fig. 37, energetic electrons detected near $4000 \mathrm{~km}$ altitude by electrostatic analyzers on the FAST satellite are shown alongside an image of 


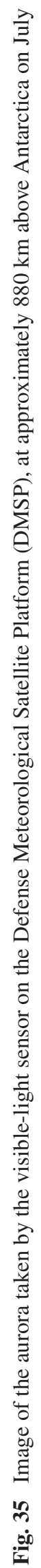




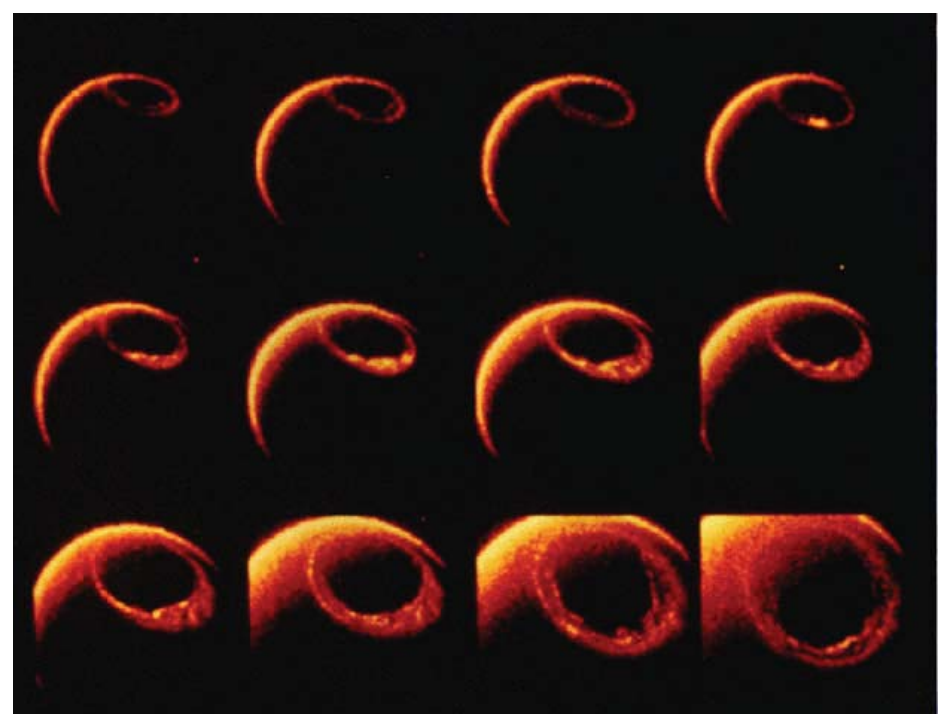

Fig. 36 Consecutive false-color images of the northern auroral oval recorded at the onset and evolution of a substorm as the Dynamics Explorer 1 spacecraft descends from near its apogee during the period 0529 through 0755 UT on 2 April 1982. Each image is telemetered in a twelve minute interval. The poleward bulge of an auroral "surge" is first seen in the fourth image, which begins at 0605 UT. A westward-traveling surge then propagates along the poleward edge of the oval as highly structured, eastward-moving auroral forms develop in the post-midnight sector. The sunlit atmosphere is visible at the upper left in each image. The passband of the optical filter is $123-155 \mathrm{~nm}$, in which the dominant responses are from emissions of atomic oxygen at about 130.4 and $135.6 \mathrm{~nm}$ and the LBH bands of molecular nitrogen (figure courtesy NASA. See also Frank and Craven 1988)

multiple auroral arcs simultaneously photographed by an all-sky camera (Stenbaek-Nielsen et al. 1998). Although the optical emissions are from much lower altitudes (near $100 \mathrm{~km}$ ), the correspondence of the visual arcs with the in situ measurements of the higher altitude electron structures is evident.

Significant progress has been made in the last 15 years establishing the relation between accelerated, energetic electrons and the discrete aurora, primarily using observations gathered from satellites that included probes with high time resolution designed specifically to address these processes, such as Viking, Freja, and FAST. As discussed by numerous authors (e.g., Carlson et al. 1998; McFadden et al. 1999; Paschmann et al. 2003; and Marklund 2009), data from these satellites have helped establish that electrostatic "Ushaped" potentials are set up in what is often referred to as the auroral acceleration region, existing at altitudes of roughly 2,000-20,000 km, high above where the electrons interact with the upper atmosphere and the auroral light is emitted. Such potential structures contain electric field components oriented along the magnetic field direction that account for the nearly monoenergetic electrons responsible for the discrete aurora. The same potential structures also accelerate ion beams along the magnetic field in the opposite direction, as observed by these same satellite probes (e.g., Shelley et al. 1976; McFadden et al. 1999).

An important feature of the data in Fig. 37 is that on the equatorward side of the event near 9:18:15 UT, there is an inverted-V region of upwards accelerated electrons. These electrons have no atmosphere in which to interact and thus no visual analog in the all-sky camera display. Indeed, the lack of optical emissions in such circumstances has been referred to as the "black aurora" by Marklund et al. (1994). High resolution satellite probes on the Viking, Freja, and FAST satellites (e.g., Marklund et al. 1994; Carlson et al. 1998; McFadden et al. 1999) have identified such upwards accelerated electrons as a regular 
Fig. 37 Multiple arcs in a low-light "all sky" image recorded on an aircraft (top) and by instruments on the FAST satellite (lower panels) near 21:20 MLT. In the top panel, the FAST trajectory is mapped along the magnetic field line to the $100 \mathrm{~km}$ conjugate point at $10 \mathrm{~s}$ intervals as the satellite passes across from left to right. The second panel is the precipitated energy flux on a linear scale. The narrow auroral arcs are clearly displayed here, as are their correspondence to the visible aurora in the top panel. The auroral optical emissions, in particular the two arcs to the far right (north), changed over the 4 minutes of the pass. The two color panels show the measured electron energy spectra for downgoing (third panel from bottom) and upgoing (second panel from the bottom) and show a number of inverted-V structures. Note the strong upgoing electrons present in the FAST data at the start of the event near 09:18:15 UT that have no visual analog in the all-sky image. The lower panel shows the FAST zonal magnetometer data corresponding to the downgoing current region, where the upgoing electrons are observed, and the upgoing current region, where the downgoing electrons are observed (after Stenbaek-Nielsen et al. 1998)

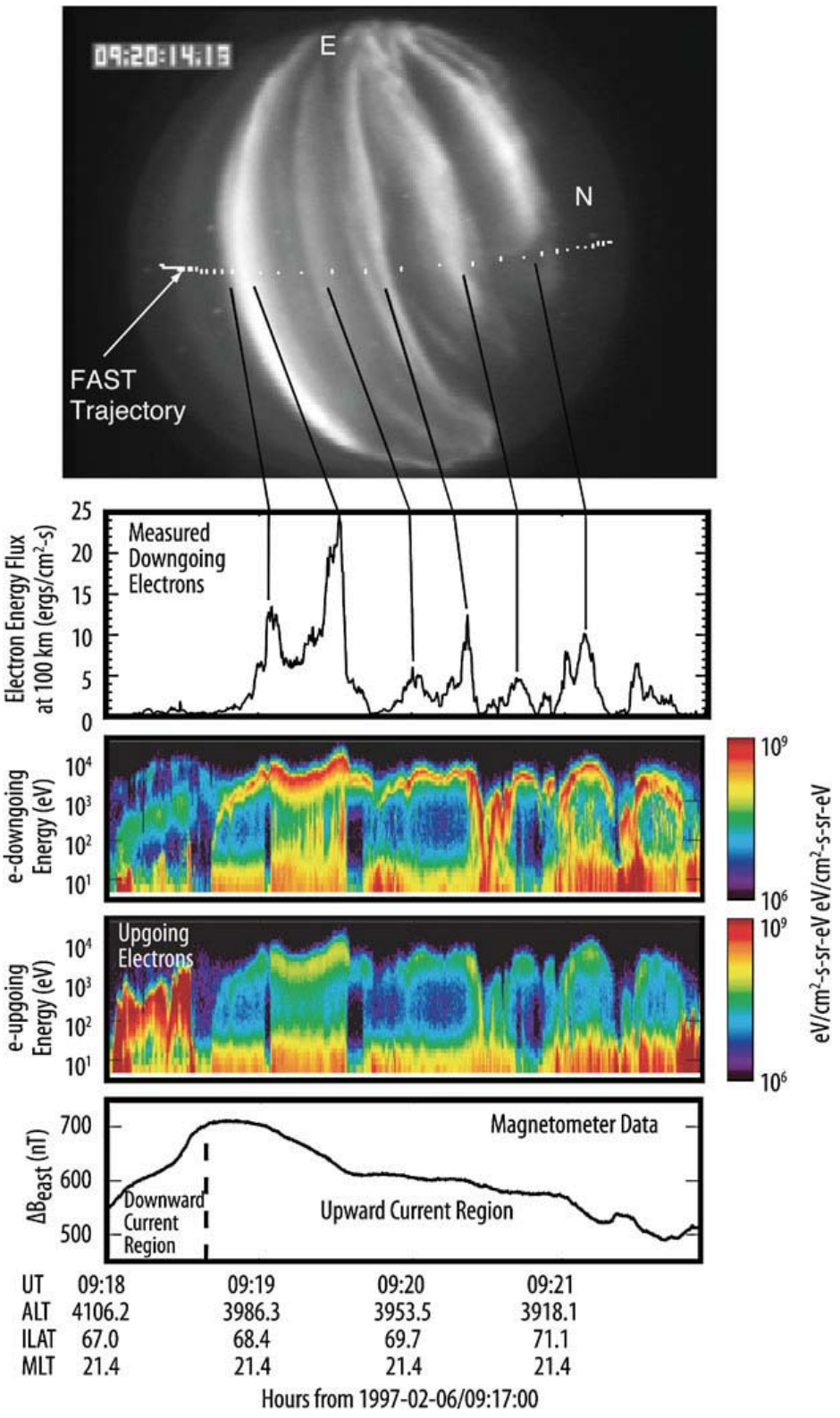

feature of the aurora. The satellite probes have also measured the perpendicular electric fields in the V-shapes and have shown how they reverse polarity in accordance with the oppositely-directed accelerated particles (e.g., Marklund et al. 1994; Carlson et al. 1998; McFadden et al. 1999). Although such upwards-directed electrons are accelerated at high altitudes, they are believed associated with current closure in the lower ionosphere, where the current is carried by lower energy electrons. The manner in which the lower ionosphere controls this current closure remains one of the fundamental outstanding problems in auroral physics today.

Oscillating Auroral Electrons Associated with Alfvén Waves. Another means by which electrons are accelerated by electric fields and subsequently precipitate into the upper atmosphere to create aurora is their interactions with Alfvén waves. In this case, the oscillating electric fields in the wave accelerate field-aligned electrons in both directions along the magnetic field which are often referred to as "counter-streaming" accelerated electrons. An 


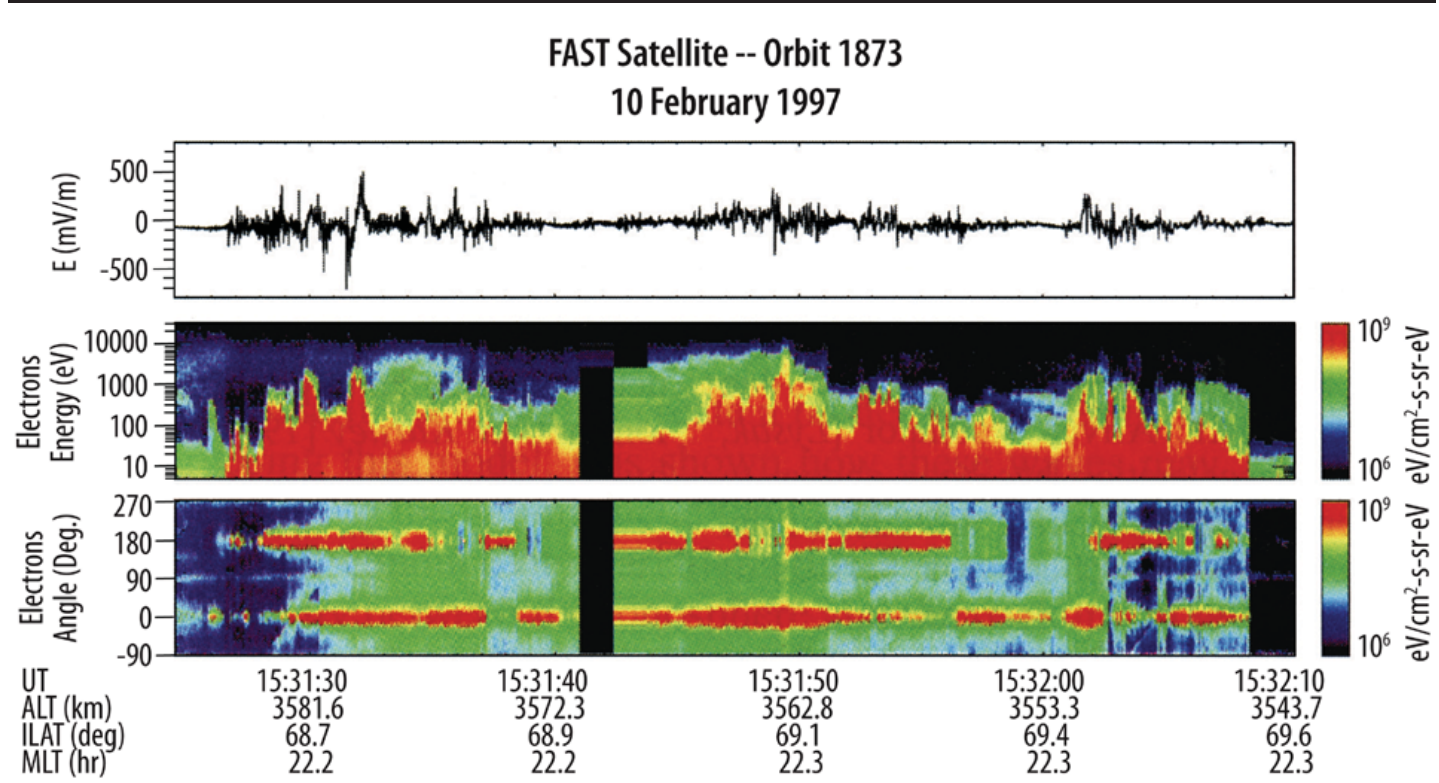

Fig. 38 Observations from the FAST satellite on the polar cap boundary. Top: the electric field along the spacecraft trajectory, or roughly north-south. Middle: the energetic electron spectra. Bottom: the pitch angle spectra of the energetic electrons with $0^{\circ}$ pointing downwards (after Chaston et al. 2004)

example gathered by the FAST satellite is shown in Fig. 38 (Chaston et al. 2004). Notice that the pitch angle information shows the electrons as highly aligned along, and anti-parallel to, the magnetic field direction, although the energies themselves are spread-out over a large range of energy space, typically between $10 \mathrm{eV}$ and $1 \mathrm{keV}$. Other detailed observations of Alfvén-wave driven field-aligned electrons, often within an inverted-V event characterized by higher energy electrons, have been reported in sounding rocket data sets (e.g., Chen et al. 2005).

Although not originally appreciated as an important source of aurora, recent observations by probes on the FAST satellite have shown that such Alfvén wave accelerated electrons can account for as much as $1 / 3$ of the total energy deposited in the ionosphere during elevated auroral activity (Chaston et al. 2007). Wygant et al. (2000) showed how the Poynting flux associated with strong electric and magnetic signatures of Alfvén waves observed on the high-altitude Polar satellite corresponded to, and was sufficient to power, intense auroral optical emissions.

Diffuse and Pulsating Aurora. Diffuse aurorae are considerably fainter than the visual aurora associated with discrete auroral arcs and are best discerned with the aid of photometers. Although such aurorae are generally without distinct, sharp features, there is some structure that can be discerned from the measurements (Lui et al. 1973; Johnstone et al. 1993). In general, the diffuse aurora is most prevalent in the post-midnight sector and has wider latitudinal extent than the discrete aurora.

The diffuse aurora may include large scale pulsations in which large patches of aurora faintly turn on and off with periods typically lasting from 1-30 seconds. Correlated with the recovery phase of magnetic sub-storms, the pulsating aurora is also prevalent in the post-midnight hours and, as with the diffuse aurora, is believed caused by wave-particle interactions with whistler mode waves (e.g., Johnstone 1983; Davidson 1990; Nishimura et al. 2010). Recent observations suggest that ionosphere-magnetosphere coupling may play an important role in the generation mechanism (Sato et al. 2004). 


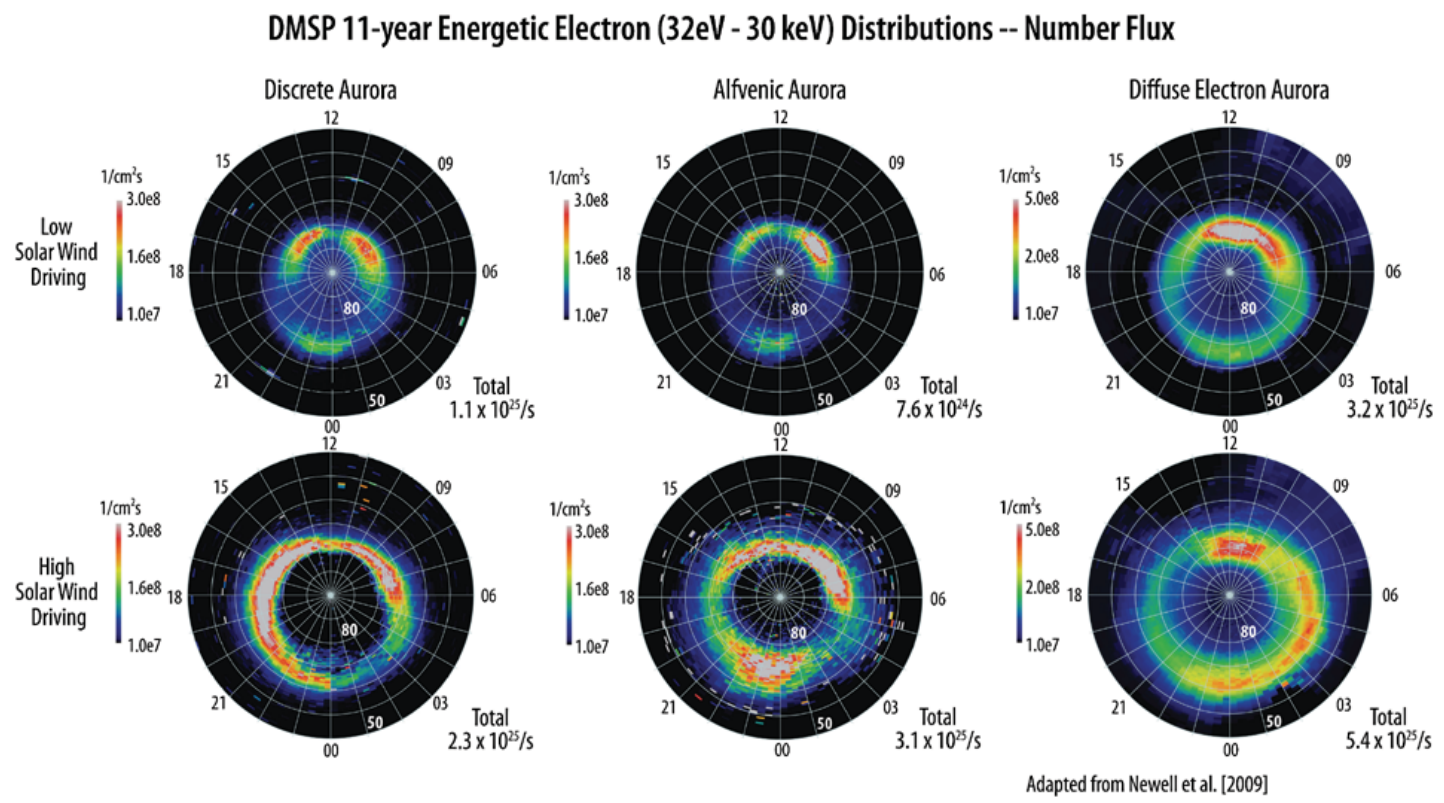

Fig. 39 Comparisons of average number fluxes of the discrete aurora (left), Alfvénic aurora (middle), and diffuse electron aurora (right), for low solar wind driving conditions (top) and high solar wind driving conditions (bottom). The data were recorded by the energetic particle instrument on the DMSP satellites over the interval of 1988 to 1998. The total number flux for each group is shown (after Newell et al. 2009). The outermost circle in each case corresponds to $50^{\circ}$ and corrects an error in the original manuscript (Newell, personal communication, 2012)

Unlike the discrete and Alfvénic aurorae which are produced by energetic electrons accelerated by components of electric fields along the magnetic field direction, diffuse aurora are caused by pitch angle scattering with whistler mode chorus waves in the magnetosphere (Thorne et al. 2010), and then precipitate when their loss cones intersect the upper atmosphere. Despite the fact that the diffuse aurora does not create as strong an optical signature, it deposits considerably more energy than the other forms of aurora, as we discuss next. Diffuse ion aurora also exists, and precipitates via the same mechanism, as discussed, for example, by Newell et al. (2009).

Relative Energy Flux of Different Auroral Processes. Newell et al. (2009) have averaged energetic electron data from the polar-orbiting DMSP satellites over an 11 year time from 1988 to 1998 . The data were binned according to the type of auroral acceleration (discrete, Alfvénic, or diffuse) and the results are shown in Figs. 39, 40 corresponding to averages of number flux (Fig. 39) and energy flux (Fig. 40). The data in each case were further binned by low and high solar wind driving conditions. As explained by those authors, the local time coverage of the satellites was fairly uniform, although the post-midnight region was covered only from 1992-1998.

The distributions shown in Figs. 39, 40 compiled by Newell et al. (2009) immediately show that discrete aurora are most prevalent on the dayside and pre-midnight portions of the auroral oval, depositing most of their energy in the post-noon, pre-midnight sector. The Alfvénic aurora is a dayside and midnight phenomenon, yet deposits most of its energy on the nightside, just prior to midnight. The diffuse aurora is primarily a post-midnight/morning phenomenon, although it is also the most prevalent form of aurora in the cusp centered at noon on the dayside. Of paramount importance is that the diffuse electron aurora is clearly the dominant source of particle energy flux into the high latitude ionosphere, constituting $63 \%$ of the energy in the low solar wind driving conditions and $57 \%$ during the 


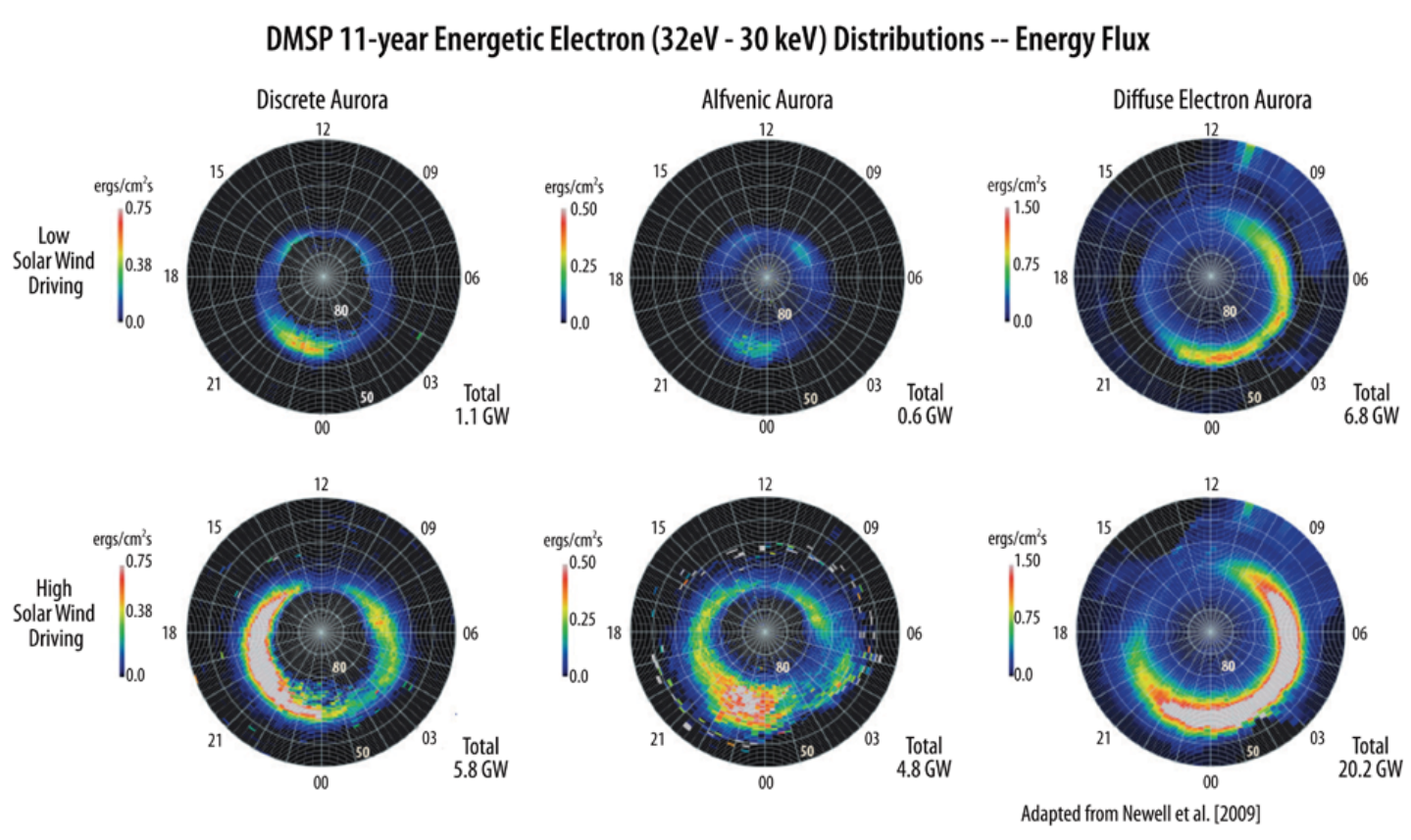

Fig. 40 Comparisons of average energy fluxes of the discrete aurora (left), Alfvénic aurora (middle), and diffuse electron aurora (right) for low solar wind driving conditions (top) and high solar wind driving conditions (bottom). The data were recorded by the energetic particle instrument on the DMSP satellites over the interval of 1988 to 1998. The total energy flux for each group is shown (after Newell et al. 2009). The outermost circle in each case corresponds to $50^{\circ}$ and corrects an error in the original manuscript (Newell, personal communication, 2012)

high solar wind driving conditions, according to Newell et al. (2009). Note that the ion diffuse aurora were also included in the statistics presented by these authors but are not shown here. That the diffuse electron aurora is so prevalent has important consequences for the conductivity of the high latitude, post midnight ionosphere, as we discuss further on below.

Cusp Aurora. The cusps are funnel-shaped magnetic field regions in the northern and southern hemispheres on the dayside of the earth directly connected with the solar wind. As such, they permit direct entry of solar wind particles and energy into the high latitude ionosphere/upper atmosphere. The cusp aurora thus represents a distinct region of the nearearth plasma at high latitudes. Indeed, the ion diffuse aurora peaks in the cusp (Newell et al. 2009).

An example of data gathered by probes on the FAST satellite as it crossed the cusp region near its perigee at $348 \mathrm{~km}$ is shown in Fig. 41. The lower panel shows the downgoing, energetic ions of solar wind origin, exhibiting an energy dispersion indicative of a cusp crossing in the presence of the southward interplanetary magnetic field (e.g., Reiff et al. 1977). The precipitating electrons are organized in very narrow, inverted-V forms with modest peak energies of 100's of eV and thus deposit their energy and plasma at higher altitudes, in the 200-300 km region (as discussed in the next sub-section). The presence of such inverted- $\mathrm{V}$ signatures suggest that local electron acceleration processes in the cusp are similar to those in the auroral acceleration region on the nightside (e.g., Yamauchi et al. 1998; Pfaff et al. 1998). The upper panel shows the highly correlated electric and magnetic field signatures, indicative of field-aligned currents and downward directed Poynting Flux present in the cusp regions (e.g., Strangeway et al. 2000; Le et al. 2002), as we will discuss later. 


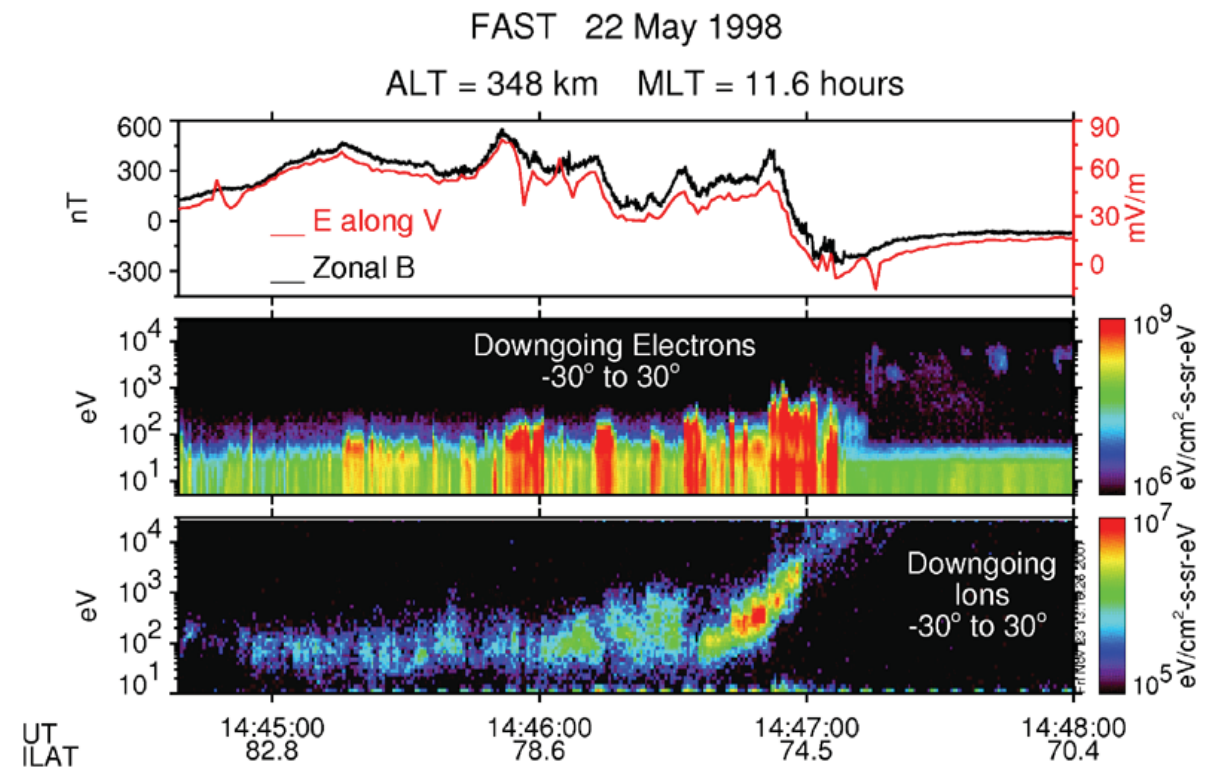

Fig. 41 Observations by instruments on the FAST satellite recorded in the cusp region near $348 \mathrm{~km}$ altitude and local magnetic noon. The bottom and middle panels show energy spectra of highly structured downgoing ions and electrons, respectively. The top panel shows the electric field component along the spacecraft velocity (red) and the zonal component of the magnetic field (black), which are highly correlated

Fig. 42 Thermal plasma production rates (left) and resulting density profiles (right) as a function of incoming electron energy (Strickland et al. 1983)
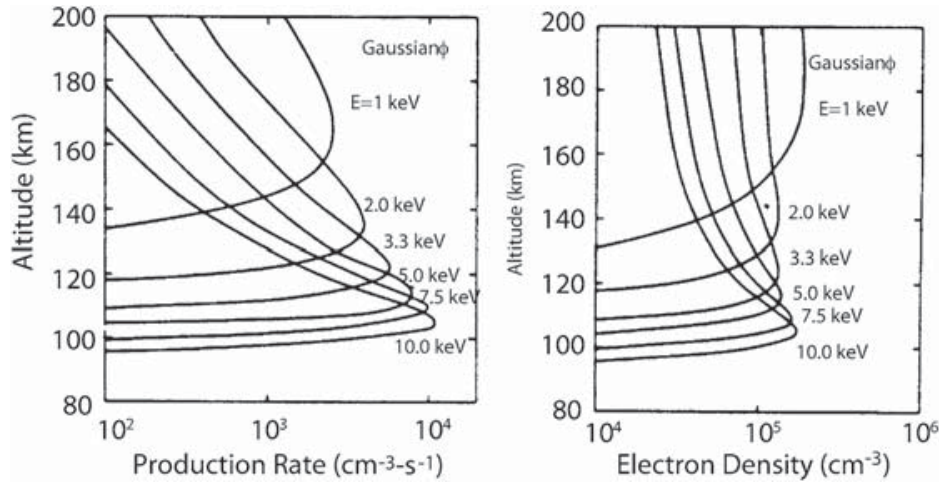

\subsection{Thermal Plasma Created by Precipitating Energetic Electrons}

The interaction altitude of the energetic electrons with the atmosphere depends on both the energy of the precipitating electrons and on the neutral density and composition of the atmosphere. The effective penetration altitude of precipitating electrons as a function of energy is shown in Fig. 42 for a model atmosphere (Strickland et al. 1983). Alongside is a panel that shows the ion production rates associated with these interactions. As with the mid and low-latitude plasma discussed previously, the molecular ions $\left(\mathrm{NO}^{+}\right.$and $\left.\mathrm{O}_{2}^{+}\right)$produced at the lower altitudes (100-130 km) recombine quickly. At higher altitudes (i.e., 200-300 km), the plasma created by the softer electrons (i.e., a few hundred eV) that ionize the $\mathrm{O}^{+}$, have much longer lifetimes and hence are important in subsequent transport and dynamic coupling to the neutral gas, as we discuss later on below.

Another example of precipitating, energetic electrons measured by the FAST satellite at $437 \mathrm{~km}$ altitude and organized in inverted-V shaped energy functions with peak energies ranging from 100's of eV to several keV is shown in the upper panel of Fig. 43. As discussed above, the inverted- $\mathrm{V}$ shapes represent spatial variations of the electron energy that occur 


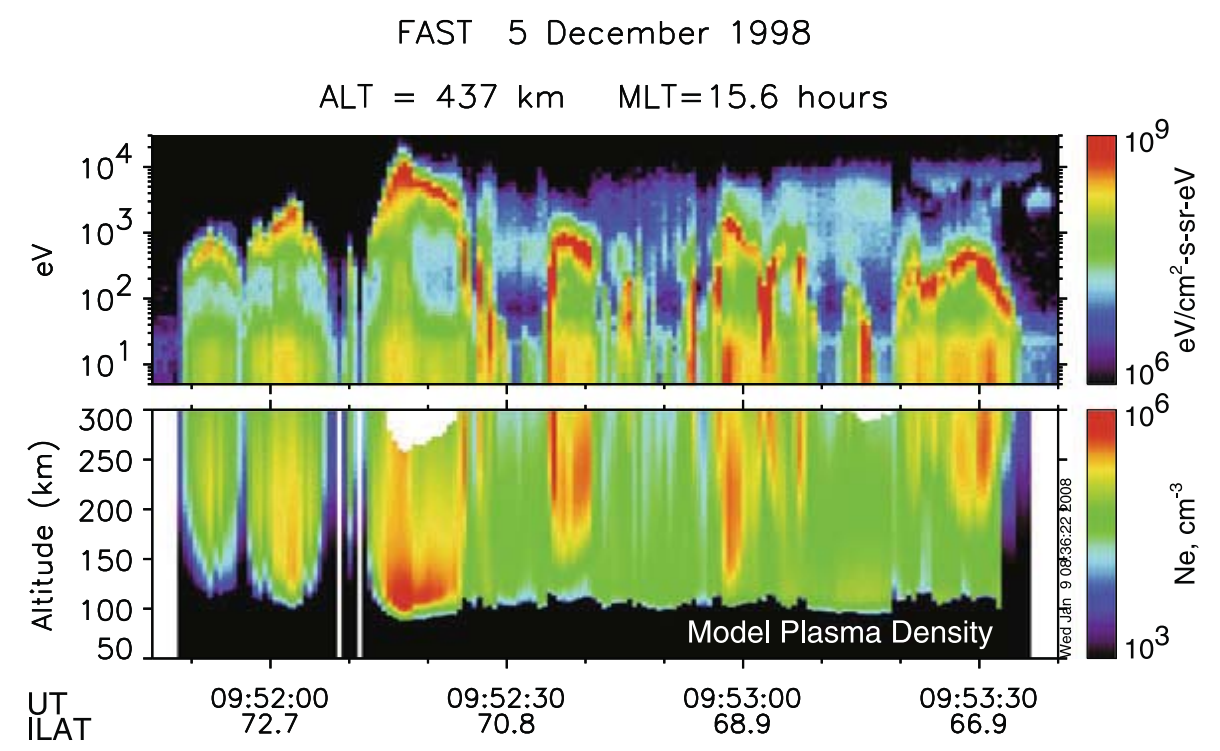

Fig. 43 Observations recorded on the FAST satellite near $437 \mathrm{~km}$ are shown in the top panel and reveal narrow beams of downgoing, energetic electron spectra. The bottom panel shows the corresponding thermal plasma created by these electrons, according to the electron transport model described in Strickland et al. (1993)

across auroral arcs. The lower panel shows the expected thermal plasma associated with these measured electron energies, according to the electron transport model described in Strickland et al. (1993). Note how the altitude of the model thermal plasma in the lower panel varies in accordance with the measured energy of the precipitating electrons.

Incoherent scatter radar observations reveal how thermal plasma created by $\mathrm{keV}$ electrons impinging on the atmosphere is indeed extended in altitude within the lower ionosphere. Figure 44 (figure courtesy I. Häggström) shows a time sequence of continuous data gathered with a fixed radar beam directed up the magnetic field line, and hence the same space-time cautions that were discussed with respect to the radar data presented in Fig. 24 must again be invoked here. These radar observations were gathered simultaneously with all-sky camera observations showing a series of "intense, high, rayed arcs", as reported by Kirkwood et al. (1988).

Diffuse aurora, which is characterized by precipitation of electrons with a broad energy spectrum (from 100's of $\mathrm{eV}$ to tens of $\mathrm{keV}$ ) over a wide latitudinal extent, can last for hours, creating substantial ionization over a wide range of altitudes (e.g., 100-300 km). The ionization at the higher altitudes persists much longer due the much longer recombination times of the $\mathrm{O}^{+}$ions, as noted above and discussed in Sect. 2.

\subsection{Characterizing the High Latitude Ionosphere Plasma}

The high latitude plasma density is directly formed via: (1) precipitating energetic electrons that create thermal plasma as they are braked by the atmosphere (as discussed above) and (2) photo-ionization. We next discuss the contribution from photo-ionization at high latitudes. In subsequent sections, we discuss how other processes must be taken into account, particularly transport via $\mathbf{E} \times \mathbf{B}$ drifts and winds, in order to specify the state of the high latitude ionosphere at any given location and time.

Photo-Ionization at High Latitudes. In the preceding sub-sections we showed how the aurora is statistically distributed within the high latitude region and discussed how the resulting thermal plasma varies as a function of altitude, depending primarily on the energy of 


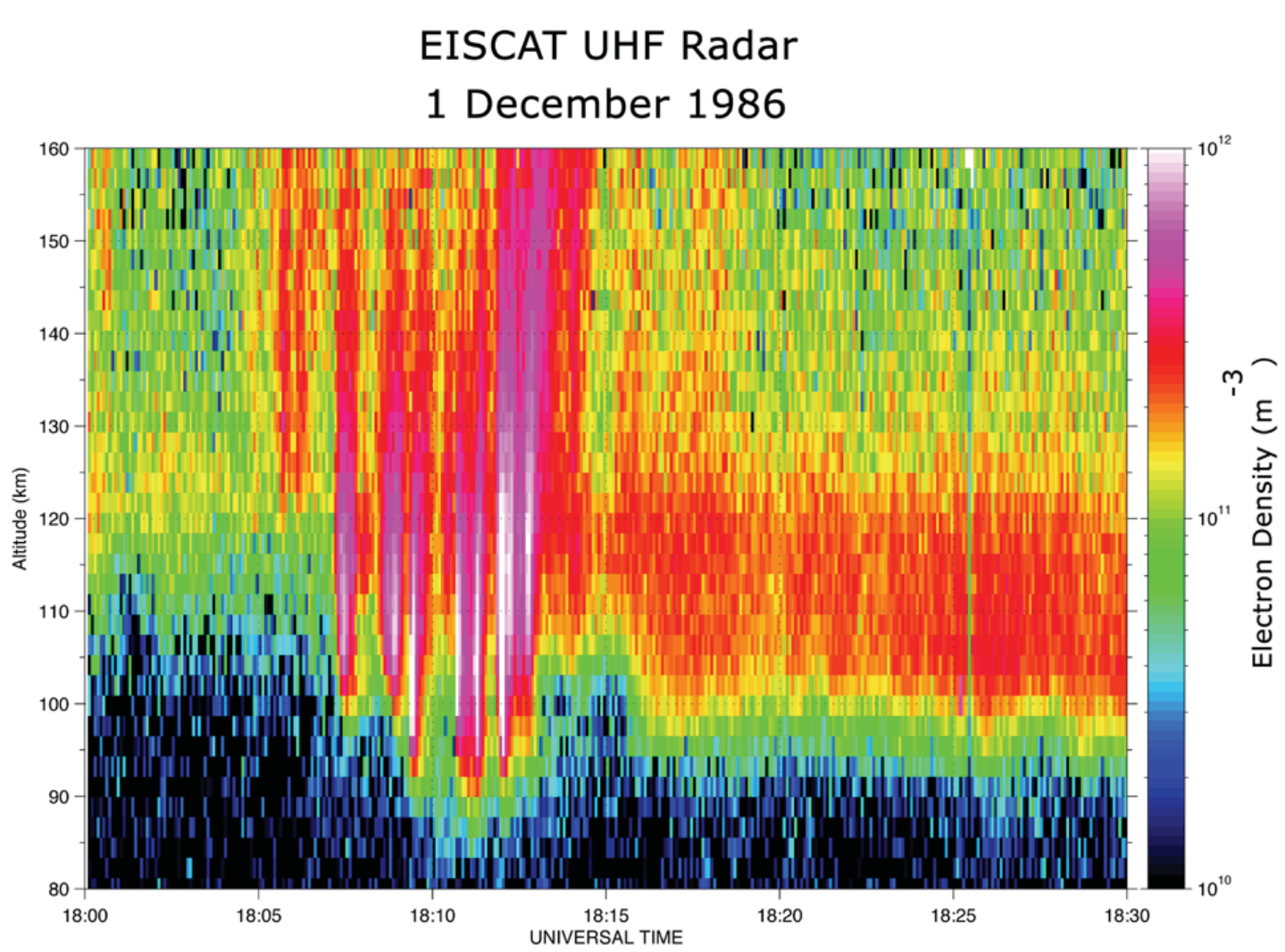

Fig. 44 EISCAT incoherent scatter radar observations of plasma density recorded with a beam oriented in a fixed position up, along the magnetic field direction (figure courtesy I. Häggström)

the precipitating electrons and the background neutral density and composition. Although ionization produced by such energetic auroral particles often dominates the local high latitude ionosphere, photo-ionization at high latitudes is nevertheless an extremely important, regular source of plasma in these regions as well.

Contributions to the plasma density from photo-ionization in the high latitude ionosphere calculated using the IRI model for summer and winter hemispheres at the December solstice are shown in Fig. 45. Although the solar elevation angle is low at high latitudes, the continuous sunlight during the summer creates a conductive E-region over the polar cap to 70 degrees on the midnight side (lower panel). During the winter, on the other hand, the opposite conditions occur, and the E-region is devoid of plasma from the midnight sector over the poles to 70 degrees on the noon side (upper panel). As with the mid- and low-latitude ionosphere, recombination efficiently diminishes the plasma population below about $200 \mathrm{~km}$ in the absence of sunlight, yet the F-region ionosphere is maintained, albeit at lower densities, even during the winter months at the pole.

Plasma Temperature and the Polar Wind. The plasma temperature in the high latitude ionosphere is highly variable due to the many different and highly dynamic processes at work in this region. Precipitating energetic electrons heat the ambient plasma to higher temperatures, although the temperature of the secondary electrons as well as heating contributions from associated local plasma waves remain controversial, as discussed in Wahlund et al. (1993). Those authors used EISCAT radar data to show that electron temperature enhancements associated with 100-500 eV electron precipitation correlated well with plasma density enhancements in the altitude region of 170-230 km. Heating via other mechanisms 
Polar Cap Electron Density -- IRI Model (2007)

22 December 2004
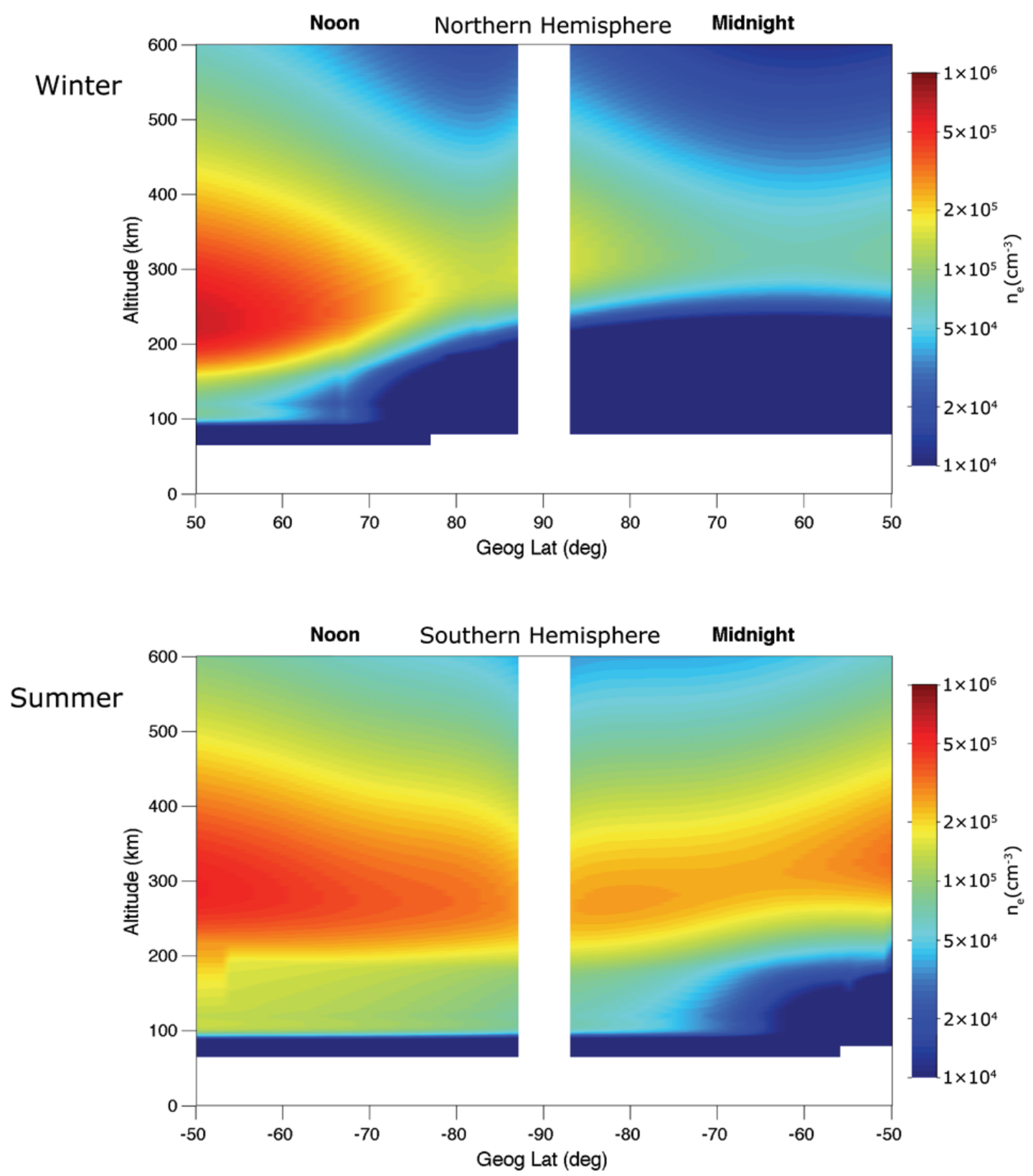

Fig. 45 IRI model calculations (for December 22, 2004) of electron density in the ionosphere starting at 50 degrees latitude, crossing the pole, and extending to 50 degrees latitude on the other side. Top: northern hemisphere. Bottom: southern. Evidence for numerical deviations in the model at the geographic pole precludes accurate estimates in this region

such as frictional Joule heating driven by perpendicular electric fields (discussed below), and plasma instabilities also create regions of increased plasma temperatures (e.g., Jones et al. 1992; Williams et al. 1992). Although this heating is initially localized, the increased pressure gradients subsequently affect neighboring regions. Despite the strong variability of the auroral precipitation within the high latitude ionosphere, the electron temperature has been modeled for different altitudes of this region by Schunk et al. (1986). 
A consequence of the larger temperatures and the nearly vertical magnetic field direction in the polar region is that plasma flows outward along the magnetic field lines, often with significant velocities. This "polar wind" is a feature of the polar ionosphere that results from an ambipolar electric field set up as the electrons rise to higher altitudes along the magnetic field lines due to their higher temperature and lighter mass, as discussed in reviews by Ganguli (1996) and Schunk and Sojka (1997). The polar wind includes both $\mathrm{O}^{+}$and $\mathrm{H}^{+}$ ions that eventually escape the ionosphere into the magnetosphere and distant tail regions. Observations of the polar wind have been measured by instruments on numerous spacecraft, including Dynamics Explorer-1 (Chappell et al. 1982, 1987; Nagai et al. 1984), Akebono (Abe et al. 1993), and Polar (Su et al. 1998). It is important to note that other forms of ion outflow, in which ions are accelerated to higher energies via wave-particle interactions, also help populate the magnetosphere with ions of ionospheric origin, as reviewed by André and Yau (1997) and others.

Structure in the High Latitude Ionosphere. The high latitude ionosphere is highly structured, which results from a variety of mechanisms too numerous to review here. (See, Kelley 2009 , for a wide-ranging discussion.) The most obvious source of plasma structure is precipitating auroral particles, as illustrated in the auroral images in Fig. 33, 34, 35 and 36. These structures can involve scales sizes from 1000's of km to less than a km. Such density forms have well-defined plasma gradients and associated electric field and current structures that drive local instabilities and produce irregularities with associated scintillations.

An important feature of the structured high latitude ionosphere is the appearance of patches of enhanced plasma density in the F-region that convect over the polar cap in the anti-sunward direction. These patches have horizontal scales that range from 100-1000 km and have associated smaller scale structure and irregularities that also produce radio scintillation effects (e.g., Tsunoda 1988; Coley and Heelis 1995). The patches appear when the interplanetary magnetic field (IMF) is southward and have been shown by numerous researchers (e.g., Buchau et al. 1985; Valladares et al. 1996) to result not from energetic particle precipitation, but rather from detached dayside F-region plasma that is subsequently transported over the polar cap as part of the general anti-sunward flow pattern (discussed below). This narrow, sunward stream of enhanced dayside aurora is referred to as a "tongue of ionization" and is strongest when magnetic activity is high (e.g., Sojka et al. 1981; Middleton et al. 2005; Foster et al. 2005).

The ionospheric plasma is particularly structured within the cusp region. Using a survey of 18 months of ELF electric field data from the Dynamics Explorer-2 satellite, Heppner et al. (1993) showed that the most intense low frequency (4-8 Hz) structures at high latitudes clearly are those in the dayside cusp region. These authors interpreted their electric field variations as either static structures or Alfvén waves. Measurements gathered along vertical profiles provided by a sounding rocket, as shown in Fig. 46, show that the plasma density is highly structured along the portion of the trajectory within the cusp proper on open magnetic field lines, yet is smooth on that portion of the trajectory that is outside of the cusp measured on closed magnetic field lines. These data were gathered in conjunction with low frequency wave electric field irregularities and are interpreted primarily as static structures that created strong scintillations that were observed on the ground during the flight (Pfaff et al. 2003).

5.4 Large-Scale Electrodynamics of the High Latitude Ionosphere: Magnetospheric Electric Fields and Field-Aligned Currents

We now discuss the electrodynamics of the high latitude ionosphere, for which we focus on the coupling of this plasma to the magnetosphere via DC electric fields and field-aligned 
Fig. 46 Plasma density along vertical profiles, measured by a sounding rocket in the cusp (lower panels). The upper panel shows how the rocket payload was launched in the cusp region from $\mathrm{Ny}$-Alesund and remained there during the upleg portion of the trajectory. The rocket then crossed from open to closed magnetic fields lines during the downleg portion of the trajectory, where the plasma irregularities and structure are no longer observed
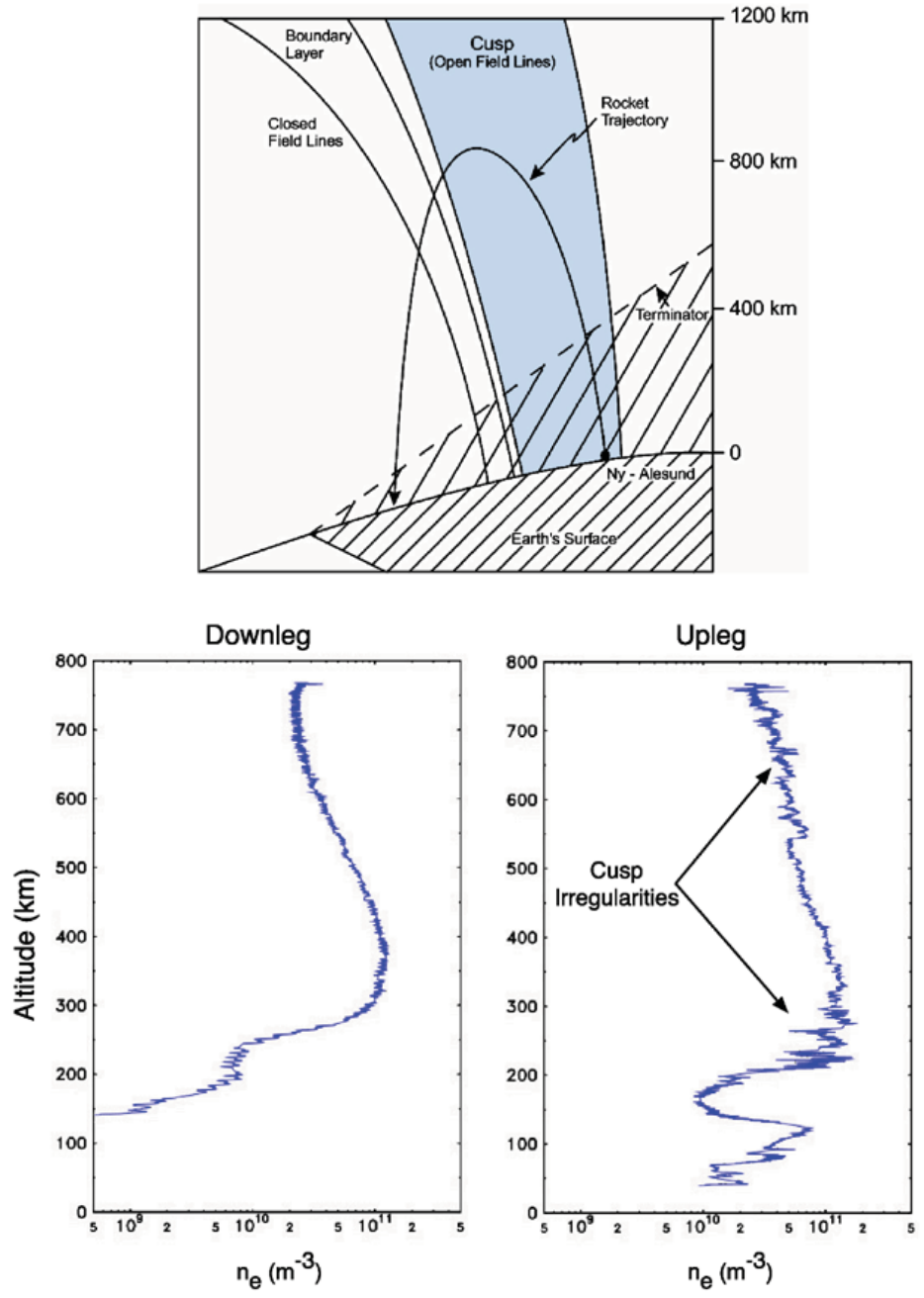

currents. Later, we introduce effects of the neutral atmosphere. Space limitations restrict our discussion here to descriptions of general features relevant to the dynamical and electrical coupling of the ionosphere and magnetosphere. For more detailed discussions, readers are referred to numerous publications on this subject, including Lyons (1992), Richmond and Thayer (2000), Cowley (2000), and Paschmann et al. (2003).

Overview of the Electrical Connection Between the Solar Wind, Magnetosphere, and Ionosphere. The solar wind is a magnetized plasma that flows around the earth's magnetic envelope, or magnetosphere. Figure 47 depicts the magnetosphere in the noonmidnight/north-south plane in which the earth is shown to scale in the center. For reference, the radius of the earth is $\sim 6400 \mathrm{~km}$. Although the near-earth plasma region that is the subject of this article would only extend a short distance on this diagram, the earth's magnetic field enables this region to communicate and exchange energy and momentum with the farthest reaches of the magnetosphere.

Since the solar wind is supersonic, a bow shock and underlying magnetosheath region is formed upstream of the compressed magnetosphere which then deflects impinging solar wind particles. The outer boundary of the magnetosphere is a current layer called the magnetopause and exists where the solar wind pressure and the earth's magnetic field pressure are equal. On the sunward side, the magnetopause is typically located at 8-12 earth radii $\left(R_{E}\right)$, depending on the solar wind dynamic pressure. 


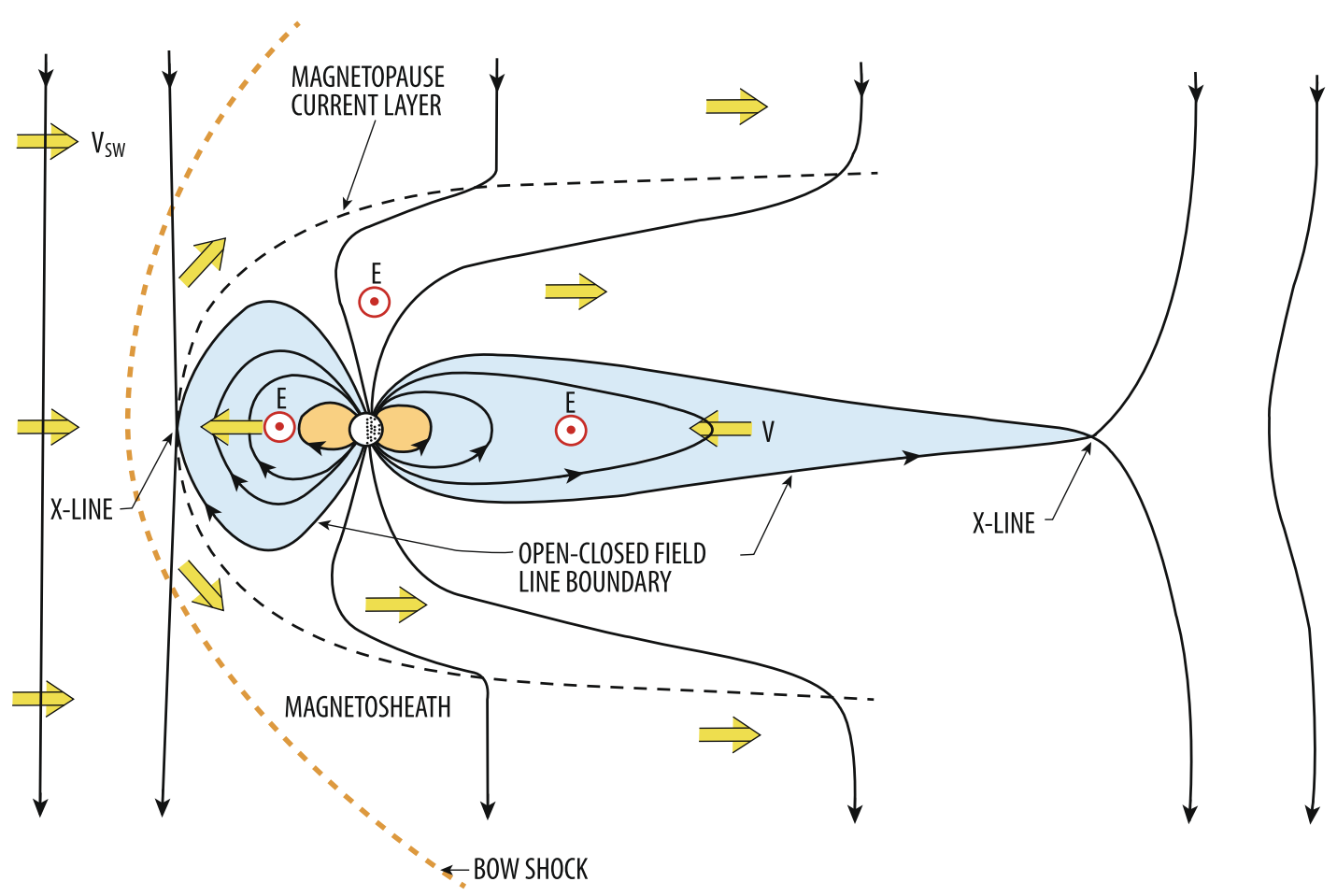

Fig. 47 The main characteristics of the magnetosphere and its interaction with a $B_{z}<0$ interplanetary magnetic field. The dawn-dusk electric field is shown, along with the principal direction of the plasma flow. The toroidal volume near the earth (shaded orange) is the co-rotating plasmasphere (adapted from Lyons 1992)

Solar wind energy connects through the magnetopause to the magnetosphere via its magnetic field. The presentation shown in Fig. 47 depicts the standard Dungey-type "X-line" configuration of interaction of the earth's magnetosphere with the solar wind with the IMF $B_{z}<0$ (Dungey 1961). (The standard coordinates used for describing the sunmagnetosphere system are: $x$, directed from the center of the earth towards the sun, $y$, directed from dawn to dusk, and $z$, directed upwards, perpendicular to the ecliptic.) The $\mathrm{X}$-line represents a merging of the magnetic fields of the earth and of the solar wind and is referred to as magnetic "reconnection" (e.g., Priest and Forbes 2000). In cases where the IMF $B_{z}<0$, significant energy is transferred from the solar wind to the magnetosphere, whereas, when IMF $B_{z}>0$, this transfer is reduced.

An essential feature of solar wind/magnetosphere interaction described above is that a dawn-dusk electric field is set up as a result of the solar wind flow across the earth's magnetic field dipole, given by $\mathbf{E}=-\mathbf{V} s w \times \mathbf{B}$. This electric field creates anti-sunward $\mathbf{E} \times \mathbf{B}$ plasma flow in the polar cap regions. The polar cap regions are essentially characterized by "open" magnetic field lines that do not close in the conjugate hemisphere of the earth, but are swept back to form the earth's magnetotail. Equatorward of these open field lines is the plasma sheet, which is a region of trapped plasma on closed magnetic field lines that are stretched down the tail and yet whose "feet" map to the auroral oval regions of the high latitude ionosphere in each hemisphere. This region corresponds to the annular regions where auroral optical emissions are most common, as shown earlier in the images in Fig. 36. The direction of the electric field within this ring in the dawn-dusk direction is opposite that in the polar cap, which is key to understanding plasma flows in the high latitude ionosphere.

To illustrate this important feature, consider the magnetosphere depicted from the perspective of looking from the magnetotail towards the sun in Fig. 48 in which only the upper 


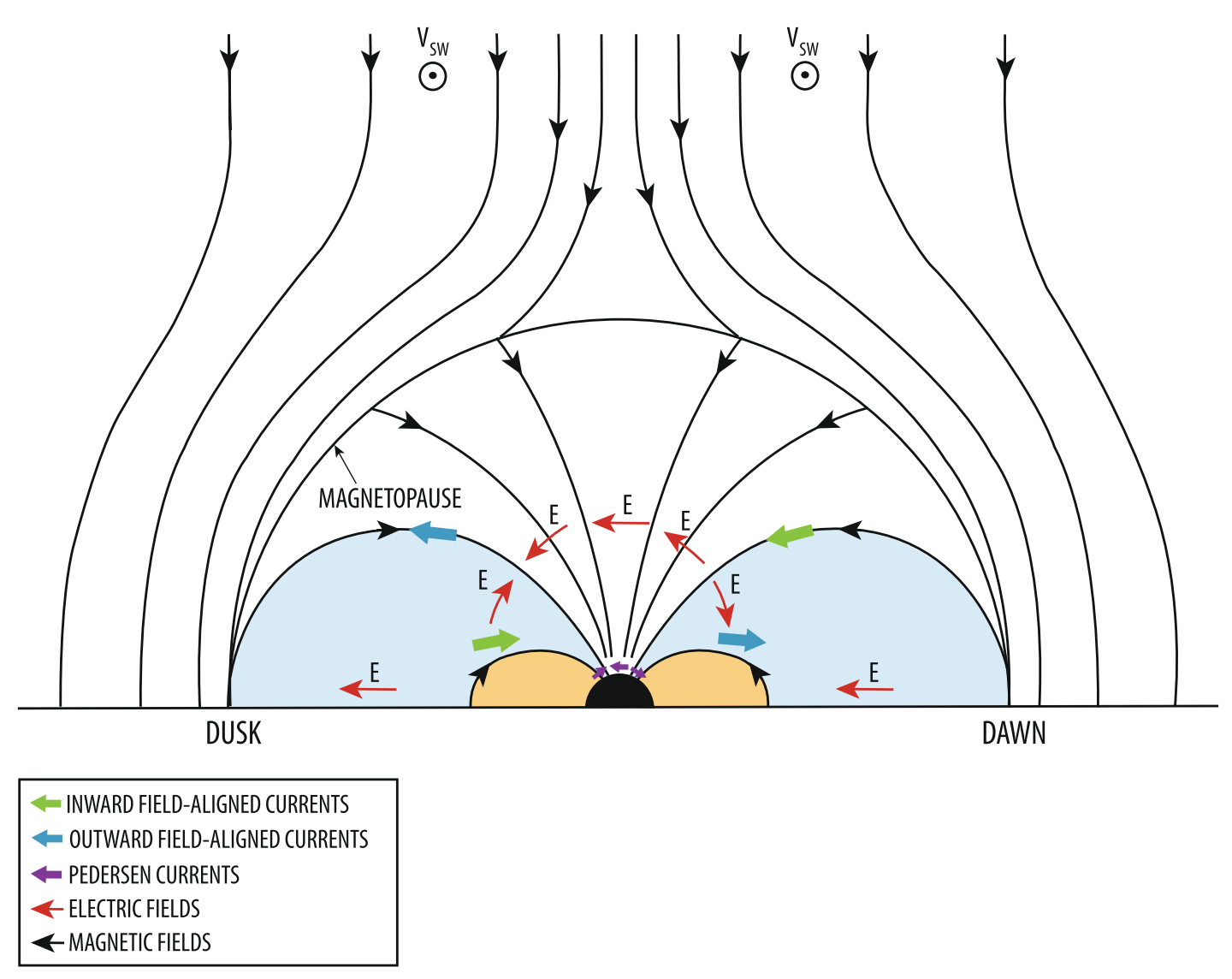

Fig. 48 The magnetosphere from the perspective of the tail, looking toward the sun. The principal current systems and electric fields are shown. The Pedersen currents with which the magnetospheric currents close in the ionosphere are generally much closer to the earth than shown (adapted from Lyons 1992)

half of the magnetosphere is shown. In the polar cap region above the earth at the center of the illustration where the magnetic field lines are essentially vertical, the dawn-dusk electric field extends directly to the earth's lower ionosphere. On the flanks of the polar cap adjacent to the magnetopause, the return plasma flow is sunward at the equator and the associated duskward-directed electric field on each side maps back (earthward) along the magnetic field lines to create an electric field oriented in the opposite direction to that in the polar cap. In this manner, we see that the electric field associated with the return, sunward flow, when mapped to regions closer to the earth, is oriented towards the pole on the dusk side and towards the equator on the dawn side. In each case, these electric fields drive sunward plasma flow in the auroral oval.

The electric field orientation and resulting plasma flow in the high latitude ionosphere is now shown from a polar perspective, in the left hand side of Fig. 49(a). The polar cap and auroral oval electric fields combine to drive a pair of vortices, or convection cells, that are responsible for the plasma circulation within the entire high latitude ionosphere. The shapes and orientations of the dual cell patterns are highly dependent on forcing by the interplanetary magnetic field (IMF), as discussed by many authors (e.g., Heelis 1988; Cowley 2000).

In addition to the patterns of high latitude electric fields, field-aligned currents of magnetospheric origin are also organized in a highly systematic manner. The principal field-aligned currents that connect the magnetosphere and ionosphere are those that flow at the boundaries of the auroral oval in each hemisphere. These currents are depicted in Fig. 49(b) where their 


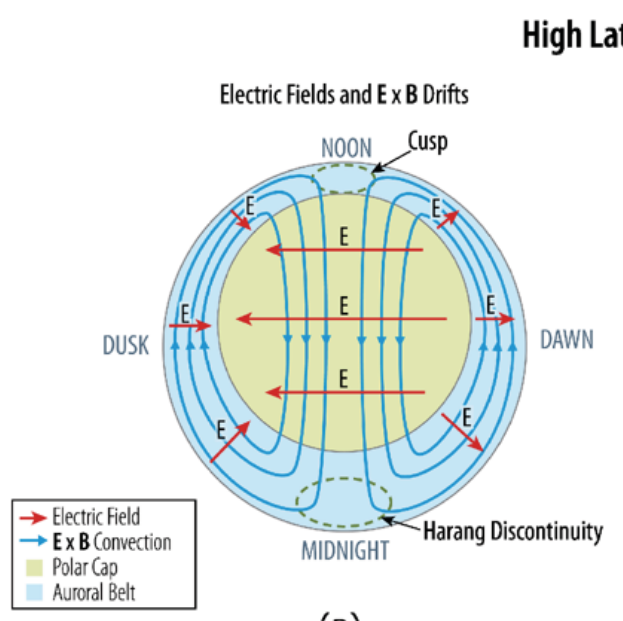

(a)

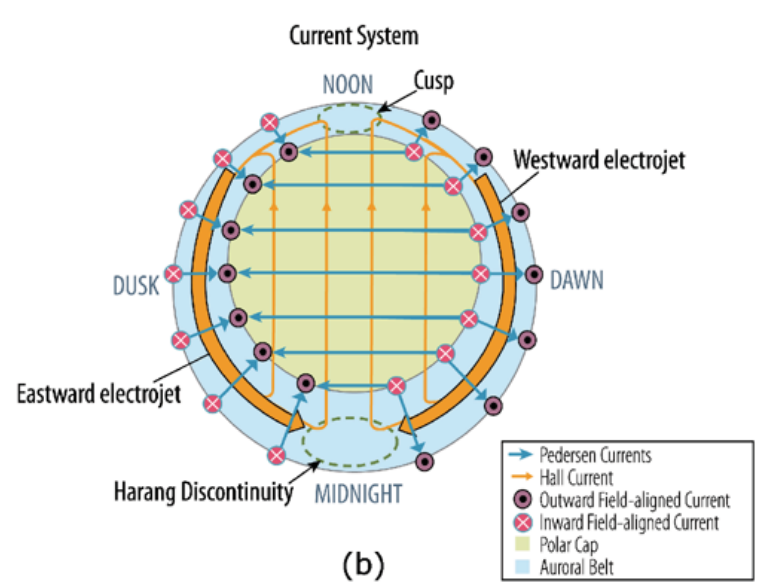

(b)

Fig. 49 The electrodynamics of the polar cap region for an idealized, symmetric coupling to the magnetosphere. The illustration on the left (a) shows the electric fields and $\mathbf{E} \times \mathbf{B}$ drifts; the illustration on the right (b) shows the principal current systems (inspired by Prölss 2004)

relation to the two-celled convection pattern and high latitude electric fields is apparent. The field-aligned currents on the poleward side (the so-called "region 1" currents) connect to the magnetopause, as shown in Fig. 48. Driven by stresses on the dayside magnetopause (e.g., Siscoe and Siebert 2006), the region 1 currents flow out of the ionosphere on the duskside and into the ionosphere on the dawn side. These currents then flow as Pedersen currents in the ionosphere and return to the magnetosphere on the equatorward side of the auroral zone, where they are referred to as "region 2" currents, flowing into the ionosphere on the dusk side and out of the ionosphere on the dawn side.

A second set of currents is driven within the high latitude ionosphere by the applied electric fields discussed above. These fields drive not only Hall and Pedersen currents, given by (6) in Sect. 3 and also shown in Fig. 49(b), but may also drive field aligned currents in the following way. If the conductivity of the ionosphere were uniform, the $\mathbf{E} \times \mathbf{B}$ plasma flows might exist without the need for a field aligned current system to maintain equilibrium. This is not the case in nature. Gradients in the conductivities force ionospheric currents to flow along field lines. Furthermore, as pointed out by Cowley (2000), the Pedersen currents flowing along the electric field direction in the ionosphere cannot close in the ionosphere, but rather a system of field-aligned currents is needed to accommodate their divergence. Note that these field-aligned currents are in addition to the principal, region-1 field-aligned currents driven by the stresses at the dayside magnetopause.

We now present experimental evidence that supports the main features of the electrical coupling between the high latitude ionosphere and magnetosphere described above. All of the data shown were gathered by probes flown on polar-orbiting satellites in the high latitude ionosphere.

High Latitude Convection. Measurements of DC electric fields and plasma drifts have been fundamental in establishing the plasma circulation in the high-latitude ionosphere. Among the earliest satellite measurements of the high latitude DC electric field are those from the OGO-6 satellite (Heppner 1972), for which an example is shown in Fig. 50. These data were gathered on a dusk-to-dawn pass of the satellite across the polar ionosphere. The large amplitude electric fields in the auroral oval which drive sunward flow are clearly visible. The polar cap electric fields are considerably weaker, but clearly correspond to antisunward $\mathbf{E} \times \mathbf{B}$ flow. 


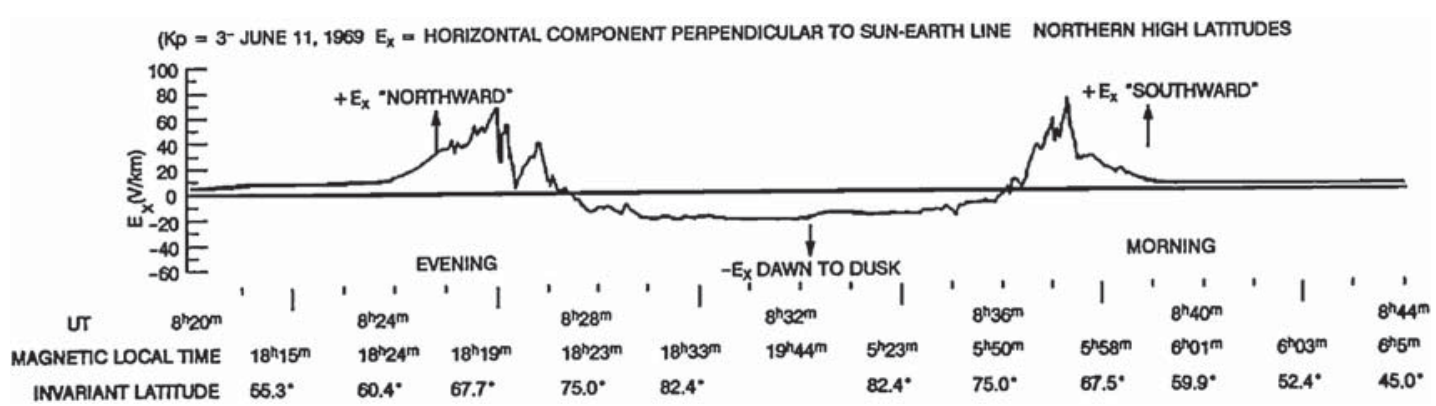

Fig. 50 An example of the horizontal DC electric field component perpendicular to the sun-earth line, observed along the dusk-dawn meridian from a low altitude pass of the OGO-6 satellite over the northern polar cap (Heppner 1972)

Direct measurements of the DC electric fields and plasma drifts in the high latitude ionosphere by numerous authors including Heelis (1984), Heppner and Maynard (1987), and others have shown how high latitude convection changes in strength and pattern as a function of the IMF. In the case where the IMF $B_{z}<0$, the plasma drifts are not only stronger, but they also show a much more organized structure with two well-defined cells that may be skewed dawnward or duskward according to the direction of the IMF By component. In cases where IMF $B_{z}$ is $>0$, the convection is not well organized, and the observed fields (drifts) are weaker. In fact, during such weakly driven conditions, the potential patterns are such that additional, isolated convection cells may be present (Heelis et al. 1986).

Models of the high latitude convection patterns, such as those based on ionospheric electric fields measured by the Dynamics Explorer-2 satellite (Weimer 1995) show how the two-celled patterns respond to the interplanetary magnetic field, as shown in Fig. 51. This particular presentation shows the potential patterns, which correspond to plasma convection flow lines. These patterns underscore how the general flow of the high latitude ionosphere is governed by the interplanetary magnetic field.

Field-Aligned Currents. In concert with large-scale patterns of magnetospheric electric fields, measurements of field-aligned currents have helped to confirm the basic magnetospheric energy flow processes discussed above. The charges carried by the precipitating energetic particles that create the aurora discussed in previous sub-sections have an associated current that flows along the direction of the magnetic field. Although such field-aligned currents were first proposed by Birkeland (1908) with respect to the aurora, their existence was not fully accepted until space-based magnetometer measurements showed their presence definitively (Zmuda 1966; Cummings and Dessler 1967).

The large-scale field-aligned currents that flow between the ionosphere and magnetosphere are not necessarily associated with optical aurora displays. In fact, these currents, which are almost always present, may be carried by low energy electrons that would not necessarily have sufficient energy to create the impact ionization or optical emissions discussed above. By convention, the field-aligned current is designated upward (downward) for downgoing (upgoing) electrons.

In situ magnetometer data from polar-orbiting satellites revealed that such field-aligned currents are typically observed as a pair of infinite sheets, providing important clues regarding how the majority of the currents might close. The upper portion of Fig. 52 depicts field-aligned currents as flowing vertically along an infinite current sheet that is traversed in the normal direction by a spacecraft carrying a vector magnetometer. The left hand illustration of a single, upward current would produce a step function in the east-west (or 


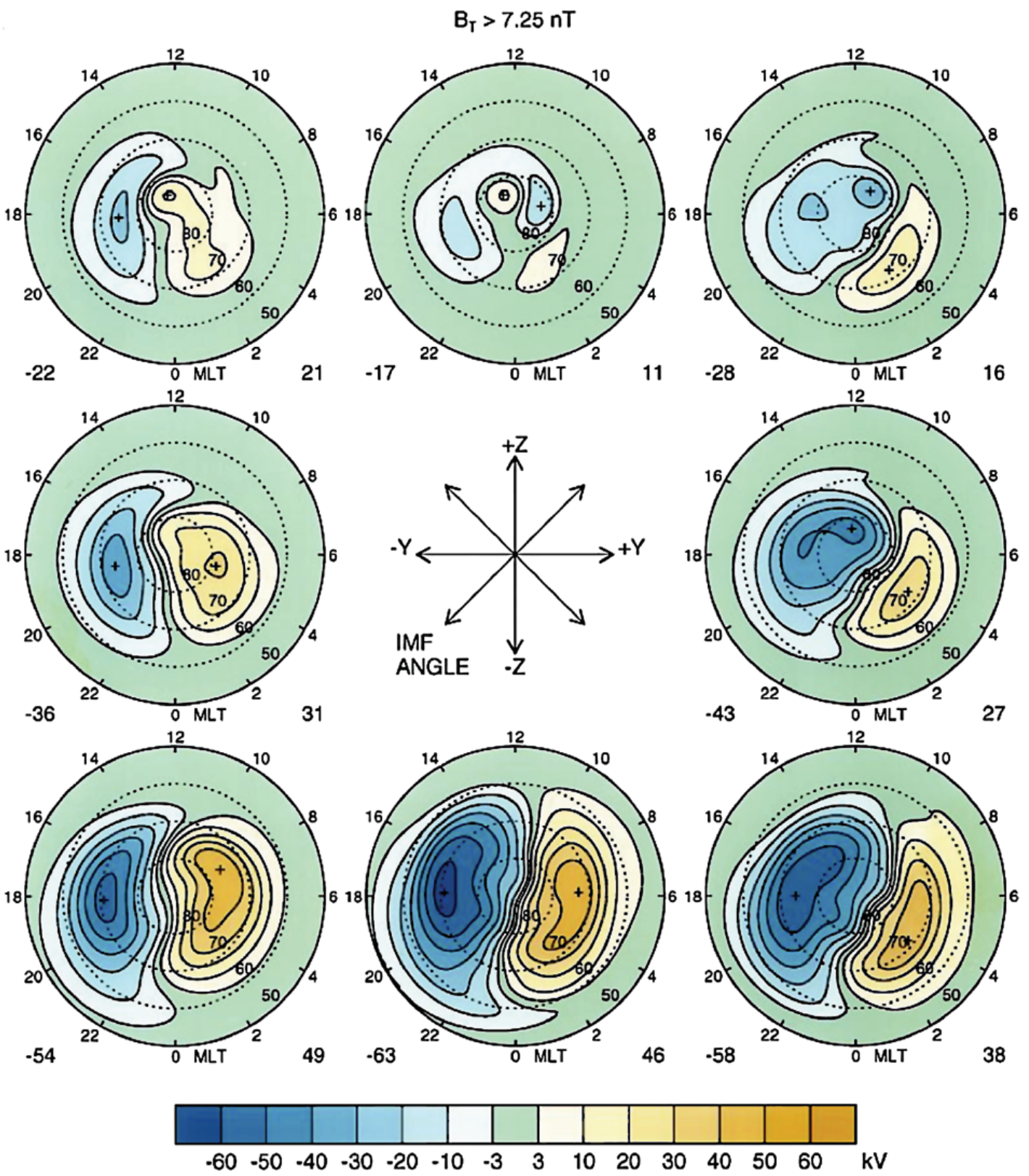

Fig. 51 Models of the high-latitude electric potential as a function of the interplanetary magnetic field (IMF), using spherical harmonic coefficients derived from measurements of the electric field by the Dynamics Explorer-2 satellite (Weimer 1995). The models shown here are for cases in which the IMF magnitude, $B_{T}$, was greater than $7.25 \mathrm{nT}$. See Weimer (1995) for an explanation of details on the figure panels

zonal) component of the satellite magnetometer data. The right hand side shows the magnetic perturbation from two anti-parallel sheets. Magnetometer data from the MAGSAT satellite gathered along a dusk-to-dawn polar orbit are shown in the lower portion of the figure (Zanetti et al. 1984). The observed zonal magnetometer component is consistent with two parallel current sheets of oppositely directed vertical currents encountered on each side of the polar cap. Note that the observed magnetometer signature appears of opposite sign when converted to east-west coordinates on the equatorward-bound satellite trajectory. 
Fig. 52 (Top) How data from the east-west component of a vector magnetometer along a polar orbit can be used to deduce field-aligned currents in single and dual parallel current sheets (Pfaff 1996). (Bottom) Fluxgate magnetometer data recorded on a polar crossing by the MAGSAT satellite. The measurements show two regions of parallel current sheets, with field-aligned currents into and away from the ionosphere, encountered on each side of the polar cap (Zanetti et al. 1984)
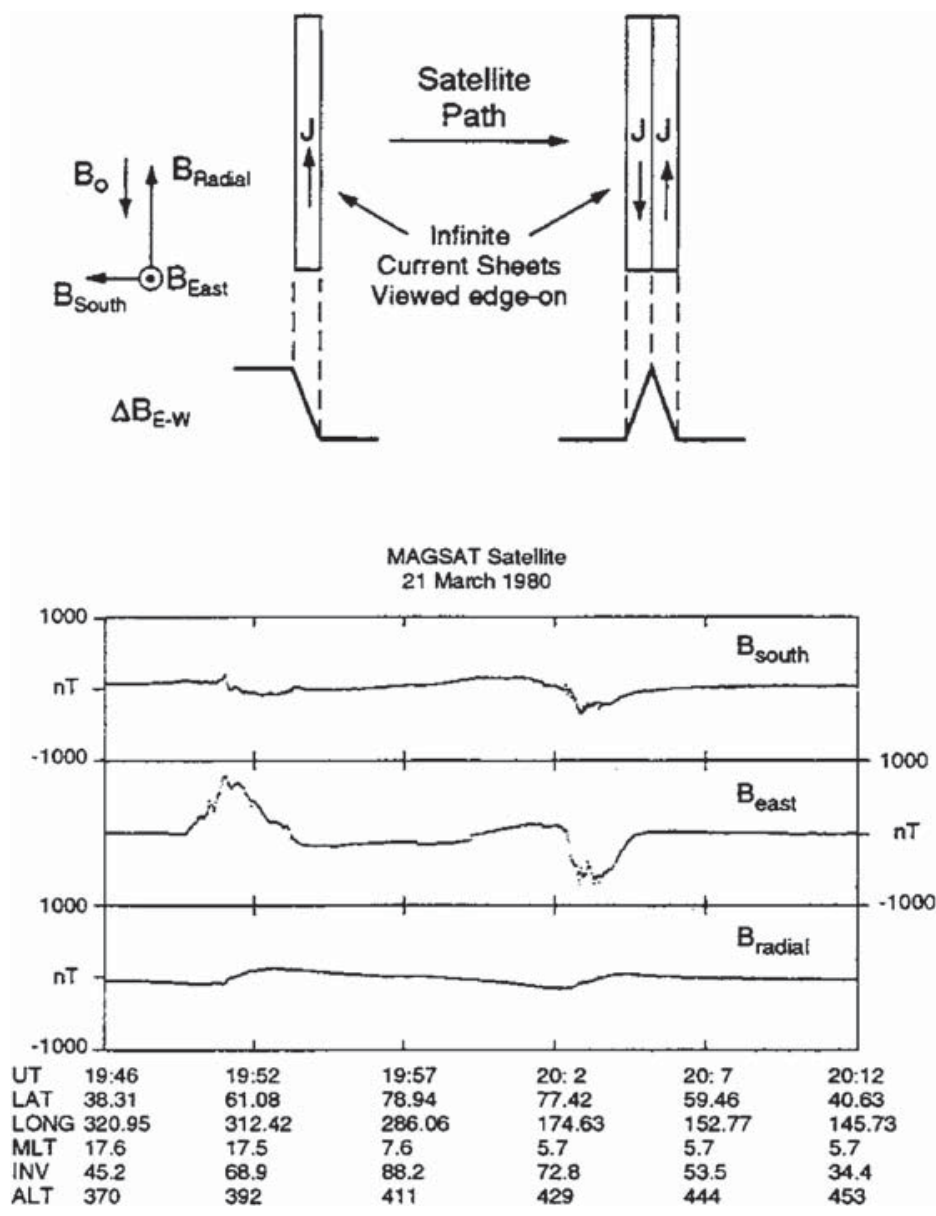

Statistical studies of these events showed that the field-aligned currents form a characteristic pattern, arranged in a belt around the polar cap corresponding to where auroral optical emissions are customarily observed, as shown in Fig. 53 (Iijima and Potemra 1976). Furthermore, the patterns show that the currents on the poleward edge of the polar cap flow into the ionosphere on the dawn side and out on the dusk side, with opposite flows on the equatorward edge of the oval. These measurements were fundamental to demonstrating the patterns of Region 1/Region 2 currents about the auroral oval that connect the ionosphere and magnetosphere, as shown in Fig. 48. Similar patterns have been recorded in other satellite data, for example in the FAST data set by Peria et al. (2000).

In cases where the field-aligned currents are carried primarily by accelerated energetic particles, detailed comparisons of data such as those gathered on the FAST satellite show that zonal magnetometer perturbations match extremely well the field-aligned currents computed from the measured fluxes of field-aligned, energetic electrons (Elphic et al. 1998). For example, returning to Fig. 41 that shows FAST satellite observations within the cusp, notice that in each instance where downgoing packets of electrons were observed, the sign of the zonal magnetic field component is negative, indicative of upgoing currents. Such measurable variations, even for extremely small electron fluxes as shown in this figure, illustrate how commonplace field-aligned currents are in the earth's high latitude plasma.

The primary manner in which field-aligned currents are believed to "close" in the ionosphere is by horizontal Pedersen currents, flowing in the direction of the ambient DC electric field, as sketched in Fig. 48 and Fig. 49(b). Although it is expected that the majority of such currents would close via a neighboring current sheet as discussed above, these illustrations 
Fig. 53 A summary of the distribution and flow directions of large-scale, field-aligned currents determined from data obtained on 493 passes of the Triad satellite during weakly disturbed conditions $(|\mathrm{AL}|<100 \gamma)$. The hatched area shown between 1130 and 1230 MLT in the polar cusp region indicates the current flow directions are not distinct (Iijima and Potemra 1976)

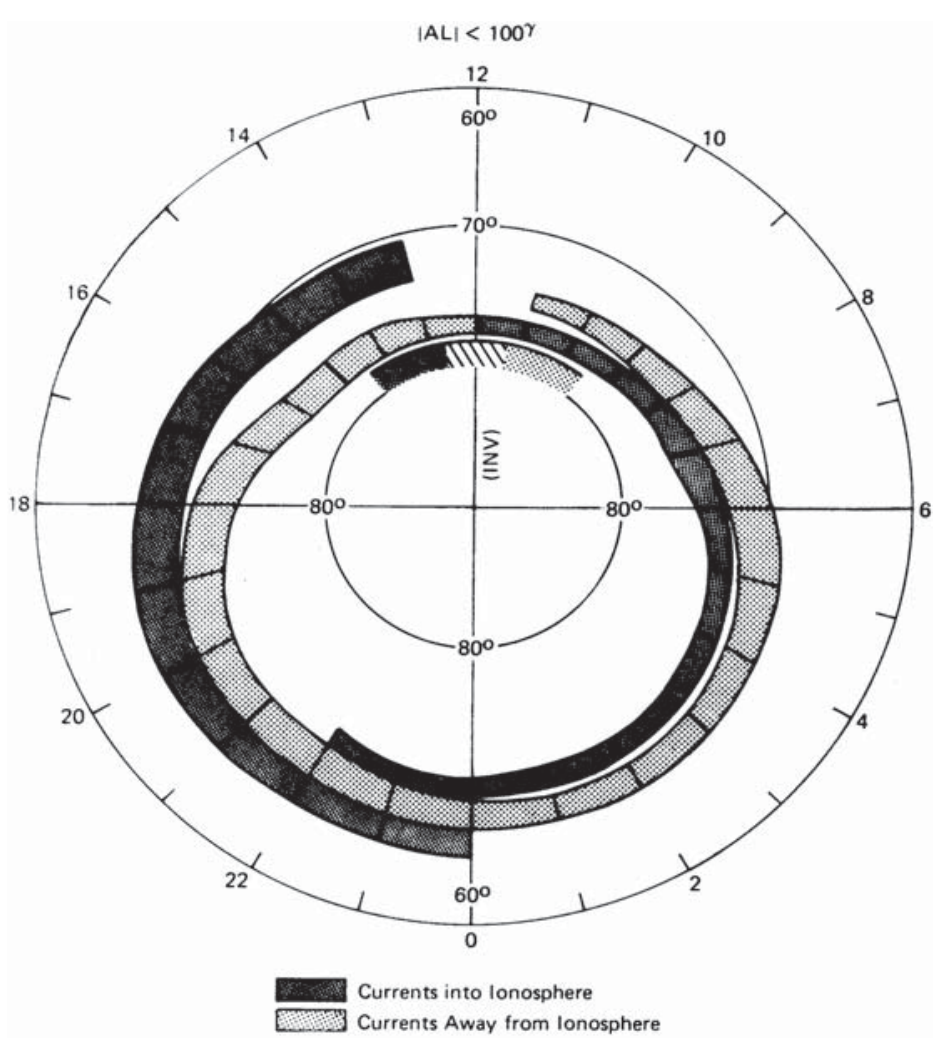

show that some of the field-aligned currents might close via Pedersen currents over the polar cap. In fact, Le et al. (2010), using ST-5 satellite data, demonstrated that such polar cap Pedersen currents were balanced by an observed asymmetry in the measured Region 1/Region 2 currents.

Closure of the field-aligned currents occurs in the lower ionosphere, where the Pedersen conductivity is largest. It is controlled both by the variations with altitude in the local thermal plasma (i.e., local conductivity), determined primarily by the spectra of precipitating energetic electrons, and by the Pedersen mobilities that depend on the charged-neutral collision frequencies and thus the properties of the atmospheric gas (see Sect. 3.2). The sunlit ionosphere on the dayside provides increased conductivity via photo-ionization to facilitate this closure.

Correlation of Electric and Magnetic Fields and Poynting Flux. Observations of DC electric fields in the high latitude ionosphere show a very strong correlation with the magnetic field variations, as shown in Fig. 54 (Sugiura et al. 1982). The strong correlation is generally present between the poleward component of the electric field, which we designate as $\Delta E_{x}$, and the eastward component of the perturbation magnetic field (i.e., in which the earth's ambient magnetic field has been subtracted), which we designate as $\Delta B_{y}$. These observations support the infinite current sheet approximation for the field-aligned currents, and is consistent with meridional closure, i.e., via Pedersen currents. Using this assumption and assuming that the Pedersen conductivity is uniform along the measurement track, the two quantities may be related by $\Delta B_{y} / \Delta E_{x}=\mu_{o} \Sigma_{p}$, where $\Sigma_{p}$ is the height integrated Pedersen conductivity and $\mu_{o}$ is the permeability of free space (e.g., Smiddy et al. 1980).

Such combined electric field and magnetic field measurements are also important for calculating the Poynting flux into the ionosphere (e.g., Kelley et al. 1991). The Poynting flux is given by $\Delta \mathbf{E} \times \Delta \mathbf{B}$ and its divergence can be related directly to the energy input to the iono- 
Fig. 54 Data from the east-west magnetic field component (after subtraction of the earth's ambient magnetic field) and the north-south electric field component measured on the polar-orbiting Dynamics Explorer-2 satellite (Sugiura et al. 1982)

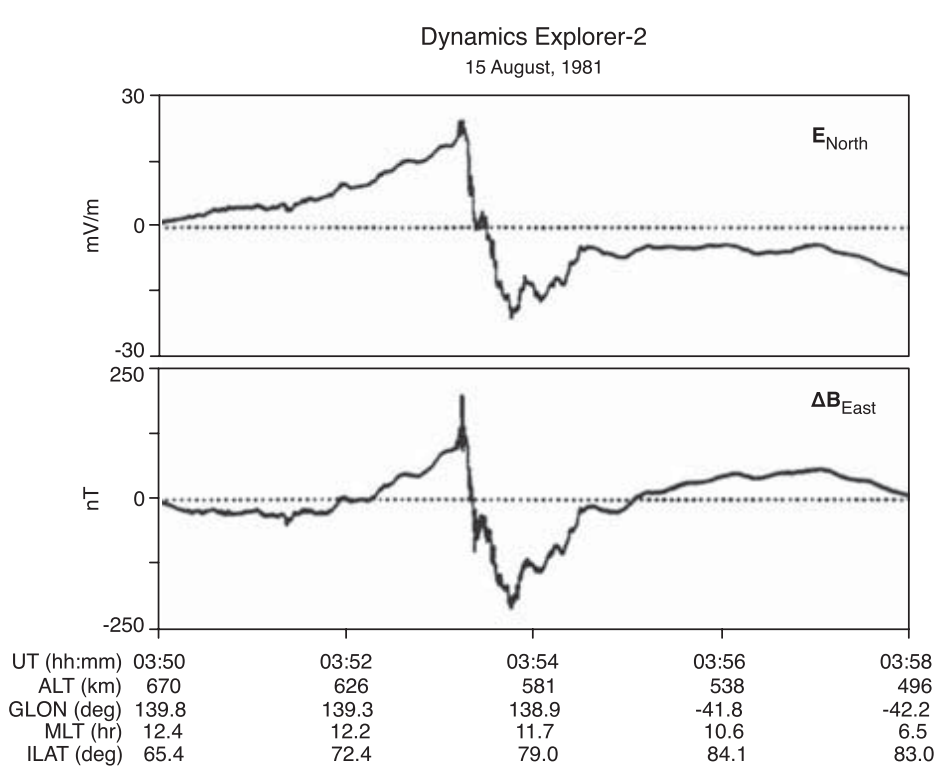

Dynamics Explorer-2

31 May, 1982


Fig. 55 Ion drift velocity (upper panel) and magnetic field (middle panel) components in the anti-sunward direction recorded by the Dynamics Explorer-2 satellite for a pass over the northern polar region. The bottom panel shows the field-aligned Poynting flux $S_{\|}=\mathbf{S} \cdot \mathbf{B}_{o}$, which is primarily from these two components. The data are from Gary et al. (1994), which shows all measured components

sphere that is dissipated as Joule heat, as discussed further in the next section. In Fig. 55, we present an example of field-aligned Poynting flux, $\mathbf{S}_{\|}$, measured by instruments on the Dynamics Explorer-2 satellite (Gary et al. 1994). In this presentation, the ion drift component is shown, which can be equated to the $\mathbf{E} \times \mathbf{B}$ component at these altitudes ( $>350 \mathrm{~km}$ ). The data shown here were gathered on a dusk-to-dawn traversal of the polar cap in which the components of the ion drift and magnetic field were measured in the anti-sunward direc- 
tion. (See Gary et al. 1994 for a detailed discussion as well as a presentation of all 6 of the measured components.) Not only does this figure again show the correlation of the crossed electric and magnetic field components, but it emphasizes how the electromagnetic energy is directly delivered to the high latitude ionosphere and upper atmosphere-i.e., downward, along the magnetic field direction.

Alfvén Waves. Alfvén waves are essentially ubiquitous in the high latitude ionosphere, as the medium is not in a steady state and motions of "slabs" of plasma (e.g., enhanced conductivity regions corresponding to auroral arcs), or shears in the electric field, will launch Alfvén waves which will carry field-aligned currents (e.g., Kelley 2009). Furthermore, Alfvén "resonators" have been proposed as regions between large gradients in the plasma density, such as between the F-region and the lower portion of the magnetosphere near (say) $3000 \mathrm{~km}$ (e.g., Lysak 1993), where Alfvén waves may be trapped. Note that such resonators need not be confined to the high latitude region.

From an experimental standpoint, following the discussion above concerning the high degree of correlation between electric and magnetic fields, we note that the electric and magnetic field signatures within Alfvén waves are also highly correlated and can mimic "static structures", particularly since the static structures can be easily Doppler-shifted by the satellite motion into the same frequency regime as the Alfvén waves. An examination of the ratios of the measured $\Delta E$ and $\Delta B$ components can be compared to the expected Alfvén velocity, $V_{A}$, which is given by $V_{A}=\left|B_{G}\right| /(4 \pi \rho)^{1 / 2}$, where, $\rho$ is the particle density which depends on the plasma number density and the ambient ion composition. A comparison of estimates of the Alfvén velocity and the integrated Pedersen conductivity (discussed above) can then be used to distinguish the origin of the correlated electric and magnetic fields (e.g., Knudsen et al. 1990). Such comparisons using in situ measurements have been discussed in detail by Gurnett et al. (1984), Berthelier et al. (1989), Knudsen et al. (1990), Heppner et al. (1993), and others.

\subsection{Atmospheric Interactions with the Magnetospheric Energy and Momentum}

In this sub-section, we introduce the role of the neutral atmosphere within the framework of the interactions of the high latitude ionosphere/upper atmosphere with the magnetosphere. We have already shown how the atmosphere brakes incoming charged particles, creating thermal plasma as well as auroral optical emissions, and have noted the atmosphere's role with respect to current closure of field-aligned currents. We now address additional processes that involve the high latitude upper atmosphere and its interactions with magnetospheric electric fields and particles:

\section{1. $\mathbf{E} \times \mathbf{B}$ Drift/Atmosphere Interactions}

- Ion drift deceleration below $250 \mathrm{~km}$

- Winds driven by magnetospheric electric fields

- Joule heating

- Electric fields driven by winds and upwards Poynting flux

- Two-stream waves driven by electrons streaming through collision-dominated ions

\section{Energetic Particle/Atmosphere Interactions}

- Creation of thermal plasma by energetic particle precipitation

- Heating due to particle precipitation

- Formation of Hall and Pedersen currents (in conjunction with the electric field) and field-aligned current closure 
Fig. 56 Ion drift velocity perpendicular to the magnetic field in the zonal (red) and meridional (black) directions, and the $\mathbf{E} \times \mathbf{B}$ drift in the zonal direction (blue), as a function of altitude for a DC electric field of $50 \mathrm{mV} / \mathrm{m}$ applied in the meridional directional and assumed to be constant with altitude. The variations of the ion drift component with altitude are largely due to ion-neutral collisions, as explained in the text. The slight variation of the $\mathbf{E} \times \mathbf{B}$ magnitude with altitude is due to small changes of the magnetic field strength with altitude

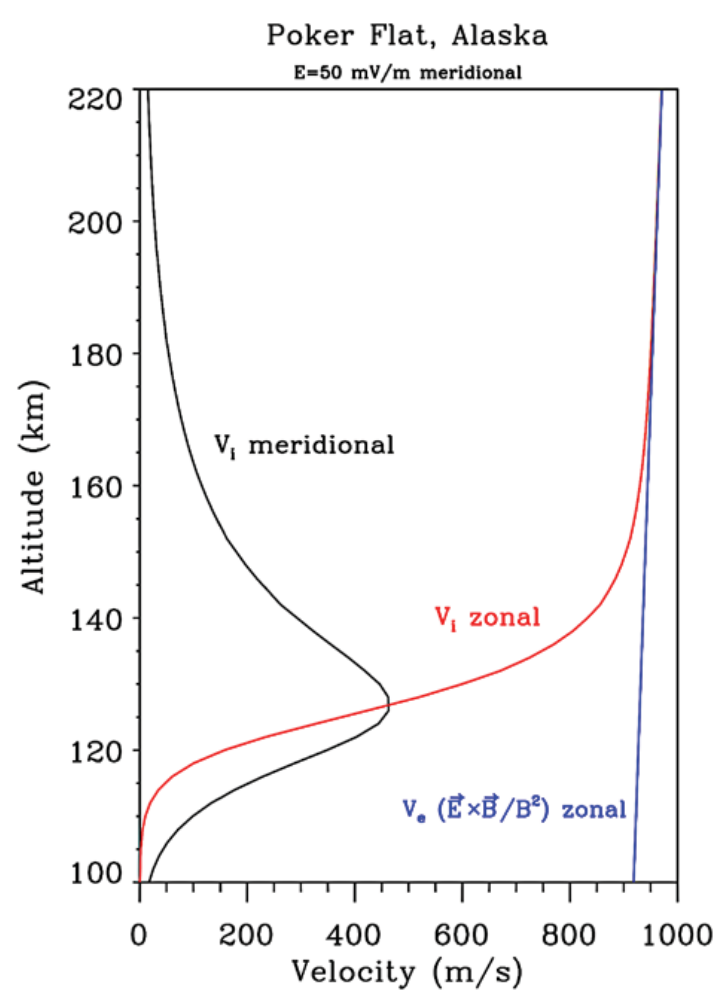

Ion Drift Deceleration Below $250 \mathrm{~km}$. As discussed above, the DC electric fields that map down from the magnetosphere set the high latitude plasma in motion via $\mathbf{E} \times \mathbf{B}$ drifts. At lower altitudes (below $250 \mathrm{~km}$ ), however, the ion motion is appreciably affected by ionneutral collisions such that the ion plasma drift is noticeably slowed and undergoes a change in direction. The electrons, on the other hand, remain fully "magnetized" and continue to execute $\mathbf{E} \times \mathbf{B}$ drifts at altitudes as low as $80-90 \mathrm{~km}$. Below these altitudes, the electron-neutral collision frequency becomes significant when compared to the electron gyro frequency, as shown earlier in Sect. 3.

The ion and electron plasma drifts, calculated versus altitude for a $50 \mathrm{mV} / \mathrm{m}$ poleward (meridional) DC electric field at Poker Flat, Alaska $(|\mathbf{B}|=0.54 \mathrm{G})$, are plotted in Fig. 56. Ions and electrons both undergo an $\mathbf{E} \times \mathbf{B}$ drift of approximately $950 \mathrm{~m} / \mathrm{s}$ at $250 \mathrm{~km}$. However, at lower altitudes, where the ion-neutral collisions become increasingly substantial, the ion drift begins to both slow and change its orientation towards the direction of the DC electric field. At about $125 \mathrm{~km}$ altitude, where the Pedersen mobility peaks, the ion drift is one half the value given by $\mathbf{E} \times \mathbf{B}$ and is equally divided between flow along and perpendicular to the electric field direction. As the ion-neutral collisions slow the ion drifts, momentum is transferred to the neutral gas, as discussed next.

Winds Driven by the Magnetospheric Convection. The strong plasma motions in the high latitude ionosphere that are driven by the magnetospheric DC electric fields set the neutral upper atmosphere in motion via ion-neutral collisions. Thus, the electromagnetic energy of the magnetosphere is converted to mechanical energy. To illustrate this coupling, Fig. 57 shows simultaneous measurements of the ion drifts (associated with the $\mathbf{E} \times \mathbf{B}$ drift) and the neutral wind measured by the Dynamics Explorer-2 satellite as it transited the polar region near $350 \mathrm{~km}$ altitude (Killeen et al. 1984). The neutral winds essentially track the ion drifts in the evening sector and within the polar cap, yet diverge considerably in the morning sector. Where the two vectors are not aligned is where Joule heating and compositional changes occur, as discussed below. 
Fig. 57 The neutral wind and ion drift vectors measured by Dynamics Explorer-2 on a perigee pass over the southern polar region, plotted in geographic polar coordinates. The neutral winds are coded by the yellow arrows, and the ion drifts are coded by the red bars. The curved line represents the location of the solar terminator $\left(90^{\circ}\right.$ solar zenith angle). The symbol $S$ refers to the location of the invariant pole at the universal time of the given pass (Killeen et al. 1984)

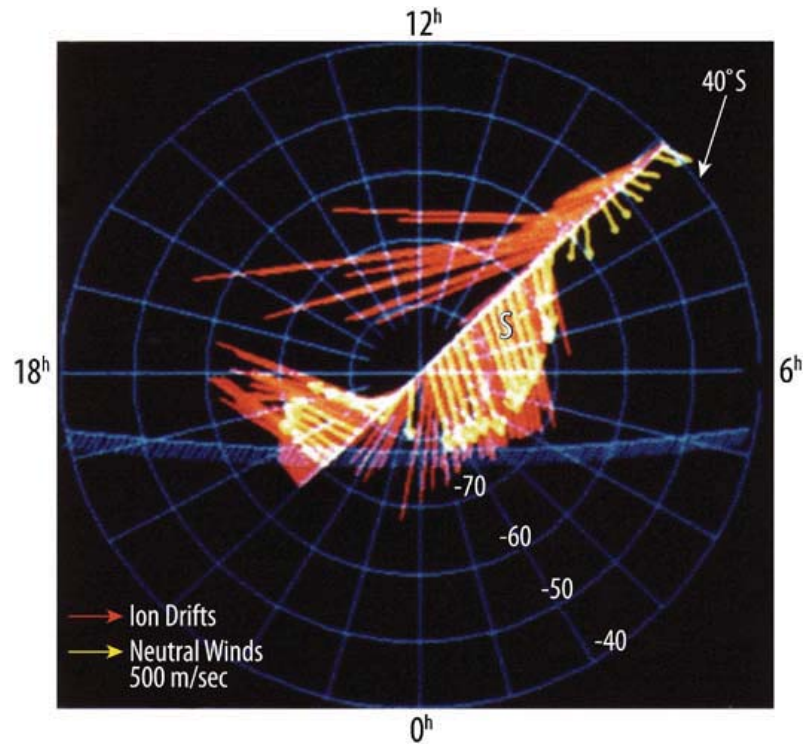

As the high latitude neutral motions are influenced by both the amplitude and direction of the $\mathbf{E} \times \mathbf{B}$ drifts associated with the magnetospheric electric fields, the resulting neutral winds show a strong and remarkable IMF dependence in accordance with the $\mathbf{E} \times \mathbf{B}$ convection, as discussed by Killeen and Roble (1988) (see, in particular, their Fig. 18).

Although the neutral wind circulation is expected to follow that of the plasma convection at altitudes of $300 \mathrm{~km}$ and above, since the ion velocities decrease and rotate with decreasing altitude below $250 \mathrm{~km}$ (see Fig. 56), the neutral wind circulation at lower altitudes is not well established. The situation is further complicated by the fact that the neutral winds are also driven by tidal forcing from below. Furthermore, during auroral events, the plasma conductance is often increased within certain altitude intervals corresponding to thermal plasma created by energetic particles, altering the coupling efficiency of the ions and neutrals. For cases where steady plasma channels might be created via diffuse auroral precipitation, atmospheric models predict that neutral "jets" may form (Brinkman et al. 1995). The existence of such jets and the neutral circulation at low altitudes associated with magnetospheric electric fields are largely unexplored in any systematic way.

Joule Heating. The relative motions of the ion and neutral drifts have a profound effect on the temperature of the neutral gas via Joule heating. Joule heating may be considered the result of friction between the ion and neutral gases that occurs when the gases are not flowing at the same speed and direction. The Joule heating rate per unit volume, $Q_{j}$, may be represented as:

$$
Q_{j}=\sigma_{p} \mathbf{E}^{\prime 2}
$$

where $\mathbf{E}^{\prime}=(\mathbf{E}+\mathbf{U} \times \mathbf{B})$ and $\sigma_{p}$ is the Pedersen conductivity, discussed in Sect. 3.2. Clearly, the Joule heating depends linearly on the plasma density and on the square of the relative motions of the plasma drifts and neutral winds. Joule heating has a strong altitude dependence due to the variation of the Pedersen conductivity and of the winds with altitude. The neutral gas heating is also affected by the fact that cooling rates increase with decreasing altitude as the neutral density increases.

To illustrate local Joule heating, we return to the example in Fig. 57, but now present in Fig. 58 other quantitative measurements gathered in situ by instruments on the Dynamics Explorer-2 satellite during this same event. The neutral winds track the ion drifts throughout 
Fig. 58 Geophysical quantities measured along the track of Dynamics Explorer-2 during the orbit shown in Fig. 20(a). The ion drifts and the neutral winds are shown in the top two traces. plotted against time, altitude, and latitude of the spacecraft. The second panel shows the electron, ion, and neutral temperatures measured along the track, and the third panel shows the atomic oxygen and molecular nitrogen number densities (left-hand scale) and the electron density (right-hand scale). The bottom trace shows the ion-neutral coupling time constant measured along the track, as discussed in Killeen et al. (1984)
Dynamics Explorer-2

21 October 1981
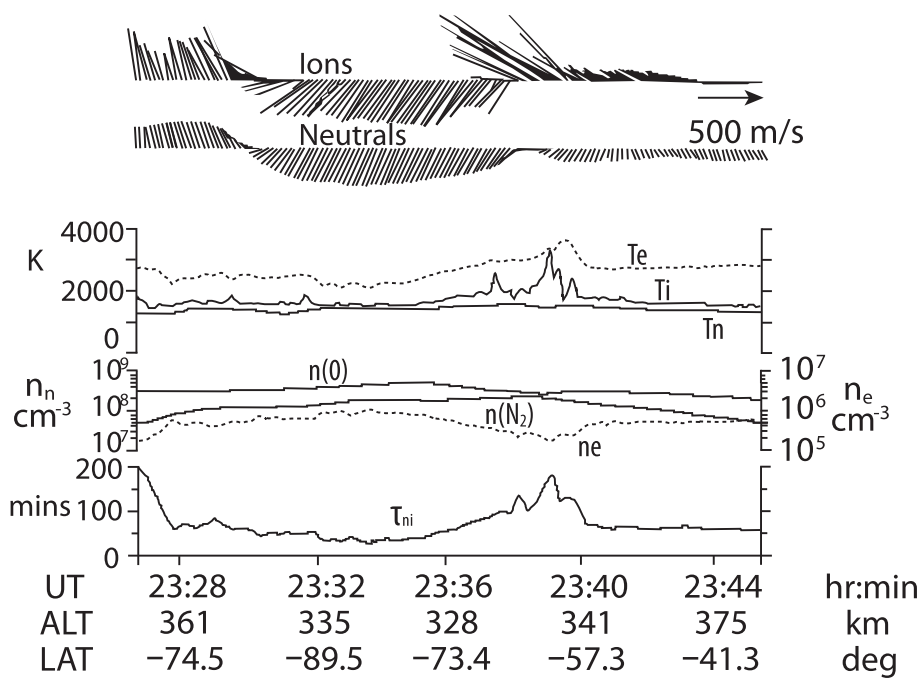

most of the satellite pass over the polar cap prior to 23:36 UT and at these times, there is little local Joule heating expected and hence there is little change in observed ion temperature. Notice, however, how the ion temperatures increase at later times when the drifts diverge (Killeen et al. 1984). This is also a region where compositional changes are observed caused by thermal expansion.

It is important to note that structured electric fields will produce significantly more Joule heating than smoothed fields that typically are used in model calculations (Codrescu and Fuller-Rowell 1995). Indeed, various researchers (e.g., Thayer 1998; Emery et al. 1999) have invoked short scale electric fields to account for a "missing energy" in circulation models and other related calculations. Joule heating represents a fundamental process whereby magnetospheric electromagnetic energy is deposited into the upper atmosphere in the form of heat, and, assuming a steady state and motionless neutral gas, can be shown to be equivalent to the Poynting flux (e.g., Thayer and Semeter 2004). Joule dissipation, frictional heating, and the related energy exchange in the ionosphere/thermosphere system have been discussed by numerous authors (e.g., Thayer et al. 1995; Thayer 1998; 2000; Strangeway 2012) and represent an important means by which the atmosphere acts as a reactive load on the magnetosphere.

Wind Driven Electric Fields and Upwards Poynting Flux at High Latitudes. The winds set up by the plasma drifts driven by magnetospheric electric fields have other profound consequences in the high latitude regions. The winds, unbound by the magnetic field, transport energy and momentum outside the regions of the plasma drifts. Furthermore, due to their inertia, they do not readily change direction as the applied $\mathbf{E} \times \mathbf{B}$ direction changes nor do they cease to flow when the driving DC electric fields are turned off. In other words, the high latitude neutral winds have persistent scales and time constants that are longer than those corresponding to the applied electric fields. Consider the case where the neutral gases are set in motion and then the driving magnetospheric electric fields are shut off abruptly. In this case, the neutral gases maintain their proper motion and generate an associated electric field that then acts back on the system. This "flywheel" effect then generates Poynting flux directed upwards, into the magnetosphere, opposite to the customary Poynting flux associated with the impinging magnetospheric energy (Kelley et al. 1991). Such "upwards" Poynting 
Fig. 59 Altitudes in the high latitude, lower ionosphere (Kiruna, Sweden) where the two-stream instability is unstable according to the linear dispersion relation, as a function of the driving DC electric field (Pfaff 1986)

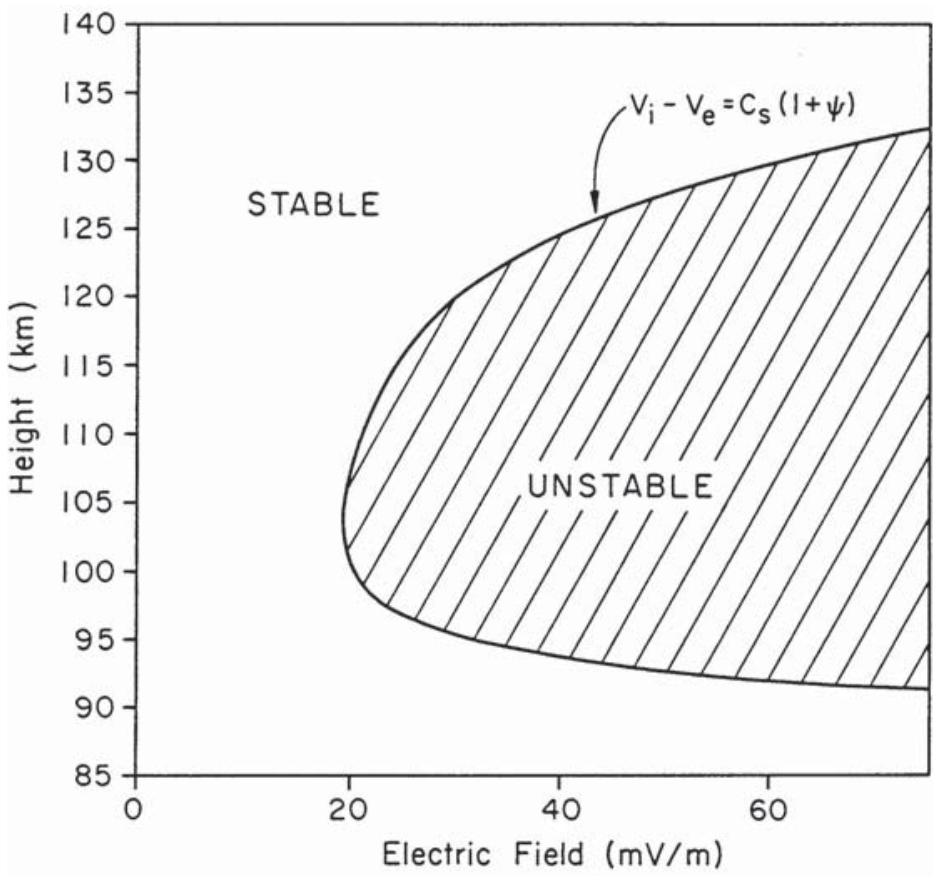

flux has been observed on the Dynamics Explorer-2 spacecraft by Gary et al. (1994) during IMF $B_{z}>0$ conditions.

Two-Stream Waves in the High Latitude, Lower Ionosphere Regions. The collisional twostream instability represents another common mechanism in the high-latitude lower ionosphere in which energy is extracted from the moving plasma driven by magnetospheric electric fields. This process occurs in the lower ionosphere, where $\mathbf{E} \times \mathbf{B}$ driven electrons "stream" through the ions that are collisionally bound to the neutral gas. A two-stream instability occurs when the relative ion-electron velocity exceeds a threshold given approximately by the ion acoustic velocity, as discussed earlier with respect to the equatorial electrojet. In the auroral electrojet, such relative ion-electron drifts occur at altitudes between 95 and $125 \mathrm{~km}$ where the Hall current is dominant. For typical magnetic field strengths at these altitudes within the high latitude regions, the instability threshold is met for DC electric fields of approximately $20-25 \mathrm{mV} / \mathrm{m}$, since the ion acoustic velocity here is about $350-400 \mathrm{~m} / \mathrm{sec}$. Although the two-stream instability is typically associated with the auroral electrojets, this condition may be met throughout the high latitude ionosphere, including the polar cap, provided the DC electric field is of sufficient magnitude.

The altitudes where the two-stream instability is generally met are shown in Fig. 59 as calculated for Kiruna, Sweden, by Pfaff (1986). The unstable region is given by the threshold condition of fluid theory (e.g., Sudan et al. 1973) given by $V_{i}-V_{e}=C_{s}(1+\psi)$. Here, $C_{s}$ is the acoustic velocity and $\psi$ is the ratio of the product of the ion-neutral and electronneutral collision frequencies to the product of the ion and electron gyro frequencies. Although $\psi$ is about 0.1 at $105 \mathrm{~km}$ at high latitudes and decreases for higher altitudes, this variable grows significantly larger at lower altitudes and accounts for the sharp cut off of the instability at altitudes of about $95 \mathrm{~km}$ and below, as shown in the figure. At the higher altitudes, the instability shuts off as the ions become magnetized and the relative ion-electron velocity is reduced. Electric field observations of two stream waves measured on a sounding rocket launched from Esrange, Sweden are shown in Fig. 60 and are described in Pfaff et al. (1992). In general, the waves correspond to meter scales and typically display large 
Fig. 60 Observations of DC electric field magnitude (left) and wave electric fields driven by the two-stream instability (right) measured on the downleg of a sounding rocket flight from Kiruna, Sweden. These data are described in Pfaff et al. (1992)

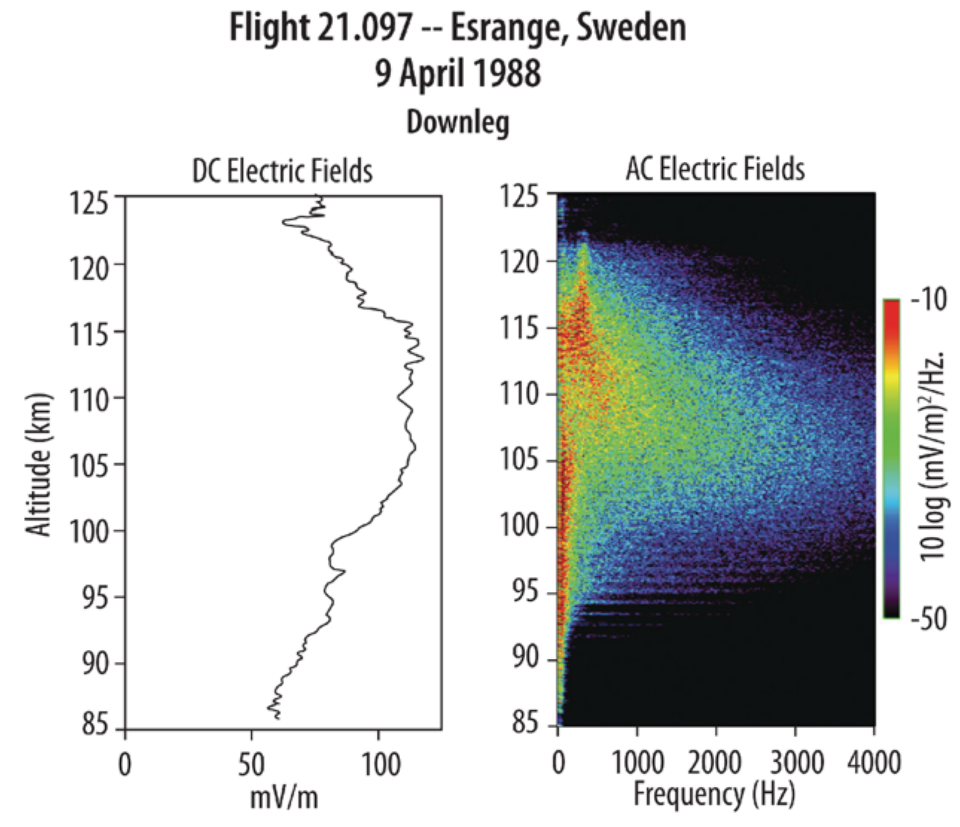

amplitudes in which the wave electric fields are a significant fraction of the driving DC electric field. Furthermore, the waves are known to heat the local electrons, raising the electron temperature by factors of 2-4 (e.g., Schlegel and St.-Maurice 1981; Jones et al. 1991; Williams et al. 1992). Although driven by DC electric fields set up by an entirely different process, the same instability is also common in the equatorial electrojet, as discussed in Sect. 4.

Creation of Thermal Plasma and Heating by Energetic Particle Precipitation. In Sect. 5.2, we discussed impact ionization and how the interactions of energetic particles of sufficient energy create thermal plasma as they are braked by the upper atmosphere. This mechanism represents a fundamental means by which the atmospheric gas and the magnetospheric plasma interact.

Absorbing the kinetic energy of incoming particles heats the neutral gas. On average, as reported by Lu et al. (1995), this heat source is about $\frac{1}{4}$ that of typical Joule heating, although it can be significant during large auroral events (e.g., Thayer and Semeter 2004). The heating caused by the mechanical energy of the precipitating particles is important for the high-latitude energy budget as well for the creation of localized effects. For example, Clemmons et al. (2008) have invoked heating by soft particle precipitation in the cusp to suggest that upwellings might explain the depletions in the neutral density measured by the Streak satellite at low altitudes.

Formation of Hall and Pedersen Currents and Field-Aligned Current Closure. In the lower ionosphere, where the ion drifts are slowed by collisions, differential drift motion of the ions and electrons drives a Hall current. As discussed with respect to the equatorial electrojet, this Hall current maximizes at the lower altitudes where the ions become essentially collision dominated. As shown in Fig. 49(b), on the nightside, the pre-midnight poleward DC electric field drives the electrons westward in the auroral oval, which results in an eastward electrojet, and the opposite is true for the equatorward DC electric field at post midnight, where a westward electrojet is driven. The electrojet currents close in the polar cap via the weaker Hall currents, which depend on both the available plasma density 
as well as the driving electric fields, and thus the current path is not straightforward, particularly where both of these quantities are highly variable. Where the currents converge in the midnight sector, known as the Harang Discontinuity, as well as in the cusp region on the dayside, field-aligned currents may be produced to keep the overall currents divergence free.

Pedersen currents are also driven by the applied electric fields, as discussed above with respect to the closure of the main Region 1/Region 2 field-aligned currents. In discussing the Pedersen currents it is important to realize that whereas the Pedersen mobility peaks near $125 \mathrm{~km}$, the altitude profile of the thermal plasma on the nightside auroral zone is highly dependent on the energy of the incoming energetic particles, as shown earlier in Fig. 43, and hence the Pedersen conductivity may peak at a different altitude than the Pedersen mobility. In other words, as the Pedersen conductivity is dependent on the combined mobility and plasma density effects, we see that the efficiency of the closure currents for a given auroral precipitation event is thus intricately tied to both the energy spectrum of its energetic particles and the characteristics of the ambient atmosphere.

\subsection{Auroral Arc Electrodynamics and Feedback to the Magnetosphere}

We conclude this section by briefly returning to the subject of discrete auroral arcs and their associated electrodynamics. Given the relevance of the aurora to the physics of the high latitude ionosphere, a few comments regarding their inherent physical processes as well as their role in coupling the ionosphere and magnetosphere are appropriate.

We showed previously how energetic precipitating auroral particles impact the neutral atmosphere to form narrow channels of enhanced thermal plasma within a limited altitude range, generally below $300 \mathrm{~km}$. In many ways, the creation of thermal plasma is simply one step in a chain of processes that includes the acceleration of the energetic particles and the feedback of the enhanced conductivity on the generation mechanism itself. The many related electrodynamic processes associated with discrete auroral arcs include: (1) the closure of the current associated with the precipitating particles, (2) the effect of the newly formed auroral arc plasma on the ambient DC electric field, (3) the enhancement of electric fields within the arc and along its sides, and (4) the feedback of the enhanced conductivity on the acceleration mechanism responsible for accelerating energetic particles. The reader is referred to the various chapters in the ISSI monograph edited by Paschmann et al. (2003) for a detailed discussion of many of these predominantly plasma processes.

Among the earliest observational results from in situ measurements gathered in the auroral ionosphere by sounding rockets is that of the anti-correlation of DC electric fields and plasma density within auroral arcs (e.g., Aggson 1969; Potter and Cahill 1969; Evans et al. 1977; Maynard et al. 1973; Mallinckrodt and Carlson 1985). To first order, such an anti-correlation is explained via Ohm's law, $\mathbf{J}=\overline{\bar{\sigma}} \cdot \mathbf{E}$. In order to maintain current continuity, the electric field is reduced where the conductivity (i.e., inside the arc) is increased. How the modified electric field effects the source potential in the magnetosphere remains an open question, particularly in cases where the conductivity of the entire auroral oval is significantly enhanced (e.g., Fig. 36). The picture is further complicated by the motions of the enhanced conducting "slabs" which affect electric fields on neighboring magnetic field lines. Several researchers have shown that auroral arcs have a proper motion that is different from that of the ambient $\mathbf{E} \times \mathbf{B}$ drifts (e.g., Haerendel et al. 1993; Frey et al. 1996). Indeed, Mallinckrodt and Carlson (1985) suggested that a larger collisional coupling with the neutrals may be necessary to explain the different velocities, and obtained a relationship between the neutral wind and the cross-arc electric field potential. 
With respect to the electrodynamics within the arc, the observed electric fields are not always decreased (e.g., Marklund 1984; Paschmann et al. 2003). The creation of polarization electric fields within auroral arcs has been explained by invoking enhanced Cowling conductivities within the arc channel (e.g., Boström 1973; Paschmann et al. 2003). Other researchers have reported enhanced electric fields and associated velocity shears at the edges of auroral arcs (e.g., Kelley and Carlson 1977). Measurements with the EISCAT radar have shown that the plasma drifts at the edges of the arcs may be in opposite directions (e.g., Haerendel et al. 1996), which may eventually help explain auroral motions such as curls and folds (e.g., Hallinan and Davis 1970; Hallinan 1976; Davis 1978).

As discussed earlier, it is now fairly well established that electric field structures (sometimes referred to as "shocks") at altitudes of several thousand $\mathrm{km}$ and higher accelerate charged particles along magnetic field lines, and that electrons accelerated downward by these structures impact the atmosphere and create the discrete aurora. These electric field structures are set up to enable the ionospheric plasma to maintain continuity of the fieldaligned currents. If the available conductivity in the volume in which the currents must flow is too small, as explained by Knight (1973), electric fields oriented parallel to the magnetic field are generated to accelerate the plasma along the magnetic field direction and hence greatly increase the available current in a given volume. (See, for example, Paschmann et al. 2003; Ergun et al. 2004; and references therein.)

Of particular importance is the discovery of "inverse" electrostatic structures of opposite polarity that account for the acceleration of upgoing electrons (e.g., Marklund et al. 1994; Carlson et al. 1998; McFadden et al. 1999). Besides direct measurements of these accelerated particles, as shown by these authors, the satellite probes have measured the perpendicular, U-shaped potentials that have the necessary reversed polarity to produce the oppositelydirected accelerated particles. The currents of these paired shocks appear to be associated with the field-aligned currents discussed above which close in the lower ionosphere. Furthermore, electrodynamic models which include low altitude acceleration of upward-directed electrons have been advanced by Doe et al. (1995) and by Marklund et al. (1997) to explain what appear to be auroral density cavities at altitudes as low as $150 \mathrm{~km}$ in the high latitude ionosphere (Doe et al. 1993). These and related observations underscore our limited knowledge of how the ionosphere participates in the acceleration of energetic particles and the closure of field-aligned currents.

To illustrate the importance of the ionosphere in understanding this fundamental mechanism of nature, consider the role of the ionospheric conductivity in the acceleration of energetic particles. Newell et al. (1996) have carried out statistical analysis of aurorae using direct measurements of energetic particle data to demonstrate that discrete aurorae are more likely to be generated in darkness than in sunlight, as shown in Fig. 61. These data support the concept that the lower conductivity of the dark ionosphere requires an auroral acceleration mechanism to be set up in order to produce the $\mathrm{keV}$ electrons to carry the current as required by the Knight relation. In a similar fashion, the abundance of the diffuse aurora in the post-midnight auroral zone may provide sufficient conductivity in the ionosphere such that the field-aligned currents are maintained without the need for potential structures to be set up to accelerate the particles. The diffuse aurora thus supplies the conductivity in the same manner as that provided by photo-ionization during sunlit conditions, in accordance with the fact that discrete arcs are not commonly observed during these post-midnight periods.

To summarize, since the ionosphere must support the field-aligned current circuit between the ionosphere and magnetosphere, its properties are essential to maintaining these 

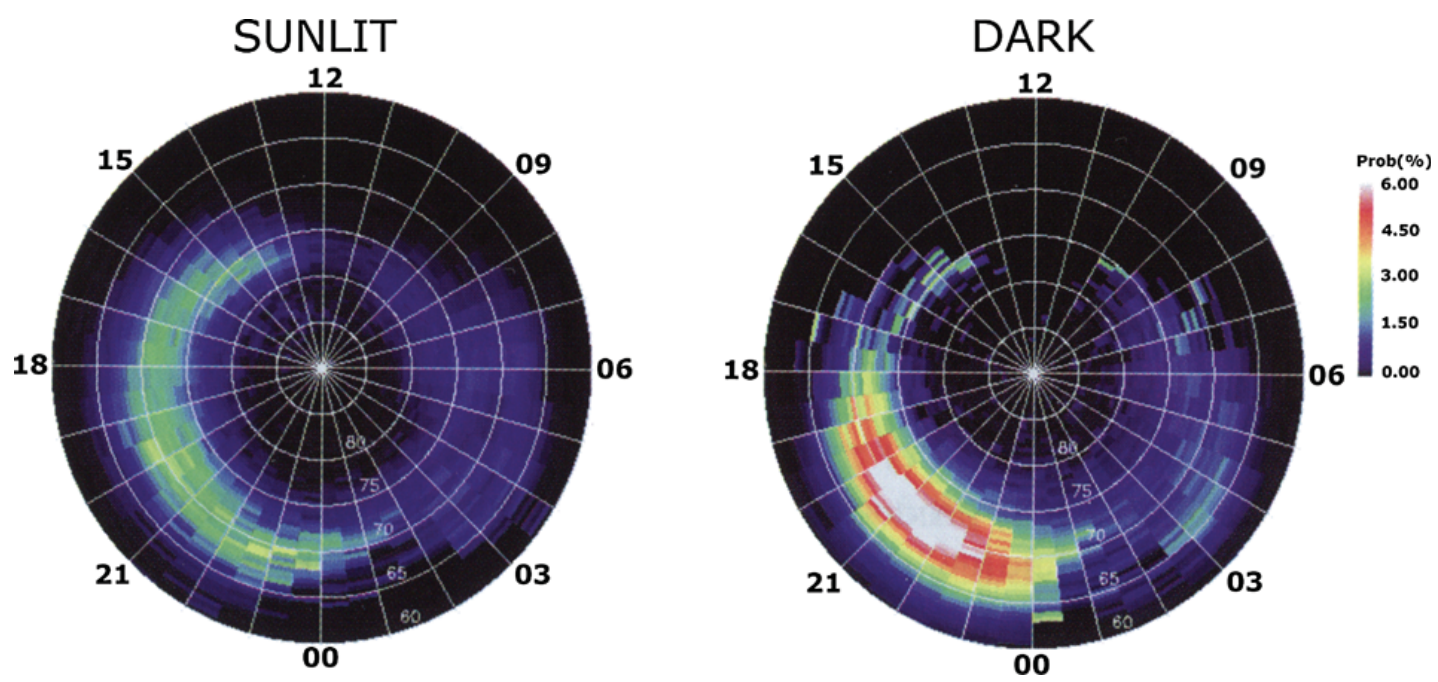

Fig. 61 Probability of observing discrete aurora when the polar ionosphere is sunlit (left) and dark (right) as a function of invariant latitude and magnetic local time, based on measurements of energetic particles recorded by the DMSP satellites (Newell et al. 1996)

currents and to the formation of the electric field structures that ultimately accelerate both the downgoing and upgoing electrons. In this fashion, the near-earth plasma plays a critical role in establishing the conditions to accelerate energetic particles which create the discrete aurora. Because the neutral atmosphere not only brakes the incoming energetic particles but also enables the high conductivity thermal plasma to form and the currents to close (via Pedersen mobilities), the critical role that the neutral atmosphere plays in the coupling of the high latitude ionosphere with the magnetosphere cannot be overstated.

\section{Ionosphere Response to Magnetic Storms}

We now provide a brief discussion of some of the ways in which the near-earth plasma responds to solar-induced magnetic activity and in particular to magnetic storms. As we shall show, the entire ionosphere including middle and low latitudes undergoes dramatic changes during the strongest magnetic storms. As we cannot describe here all of the ways in which the ionosphere responds to magnetic storms, we direct the reader to a number of reviews on this subject, including Prölss (1995), Forbes et al. (2000), and Mendillo (2006), as well as the articles in the AGU monograph on mid-latitude ionospheric disturbances edited by Kintner et al. (2008).

The near-earth plasma environment is continuously responding to two types of variations of the sun: (1) variations of the flux of solar EUV radiation, as discussed in Sect. 2, and (2) variations of the solar wind at the earth's location which subsequently influence the magnetosphere/ionosphere/thermosphere system. In this section, we focus on the second type, and in particular, the response of the near-earth plasma environment to magnetic storms. Magnetic storms are initiated by prolonged periods of the solar wind's southward $\left(B_{z}<0\right)$ magnetic field direction and are most severe when associated with a "cloud" of solar wind plasma (e.g., Gonzalez et al. 1994). Major storms may last 2-3 days and are typically monitored by the storm-time disturbance index, or Dst. Dst is calculated from magnetometer data gathered at low latitude stations that provide a measure of the magnetospheric ring current energy. Energy transfer from the solar wind to the magnetosphere results in an increase in 
the intensity of the ring current that corresponds to a negative Dst perturbation (e.g, Daglis et al. 1999). The main phase of a magnetic storm has an associated negative Dst excursion which lasts for several hours, with the largest storms displaying Dst values of $<-100 \mathrm{nT}$.

The magnetic substorm is another important category of magnetic activity to which the near-earth plasma responds (Sergeev et al. 2012). Magnetic substorms are different than magnetic storms in that they are generally much briefer, and result from energy released from the tail of the magnetosphere which then propagates into the high latitude auroral oval. Substorms are characterized by brightenings and movements of auroral displays and have strong associated high latitude magnetic disturbances that result from increased currents. The appearance of substorms as well as magnetic storms are evident in an index of magnetic activity is known as $K_{p}$ that monitors worldwide magnetic disturbances every 3-hours with an integer index from 0-9 corresponding to a logarithmic scale. A general discussion of geomagnetic indices is provided by McPherron (1995).

The influence of magnetic storms on the near-earth plasma may be considered from two general perspectives: (1) magnetic storm effects that originate at high latitudes, for example increased magnetospheric energy and momentum deposition in the high latitude ionosphere/upper atmosphere, which then have subsequent consequences at lower latitudes, and (2) global magnetic storm effects which are applied to the ionosphere/thermosphere system at all latitudes within a brief time interval after storm onset, for example those effects driven via prompt penetration electric fields. Below, we discuss examples of magnetic storm effects on the ionosphere/thermosphere system, corresponding to each category.

\subsection{Magnetic Storm Effects that Originate at High Latitudes}

Magnetospheric Energy and Particle Increases at High Latitudes. During a magnetic storm, the polar cap and auroral oval widen and the electromagnetic input and auroral emissions increase considerably. Although these changes are driven by the larger-scale magnetospheric activity, the increased energy and particle input to the ionosphere/thermosphere system is initially concentrated within the high latitude regions. For example, an estimate of increased heating at high latitudes during the magnetic storm of January 10-11, 1997, compared to quiet conditions, is shown in Fig. 62. This heating has been calculated by Richmond and Lu (2000) using a model that assimilates a variety of ground-based and space-based measurements. The upper panels show the energy input due to particle heating, whereas the lower panels show the energy input due to Joule heating. It is clear that the energy input to the high latitude region is significantly increased in both cases compared to quiet conditions and that this energy is greater for the Joule heating. As noted by these authors, the Joule heating has a greater effect on the thermospheric circulation not only because of its larger energy but also because the particle energy is deposited within an altitude region that extends as low as $90 \mathrm{~km}$ where the neutral gas, with its significantly larger density, is more difficult to perturb. The storm-driven energetic particles nevertheless provide extremely important conductivity changes via impact ionization, as discussed in the last section, as well as other effects.

Magnetospheric energy input at high latitudes during storms, such as shown in this example, can lead to significant effects at lower latitudes, as reviewed by Fuller-Rowell et al. (2008). For example, strong pressure gradients caused by the enhanced Joule heating in the high latitude regions during active periods drive strong equatorward neutral winds that are not constrained by the magnetic field. Neutral wind changes driven by a sudden increase in Joule heating at high latitudes have been computed via the CTIPe numerical simulation and are shown in Fig. 63 (Fuller-Rowell et al. 2008). The winds are shown at 15 UT for the time corresponding to three hours after the onset of the high latitude forcing. Note the wind 


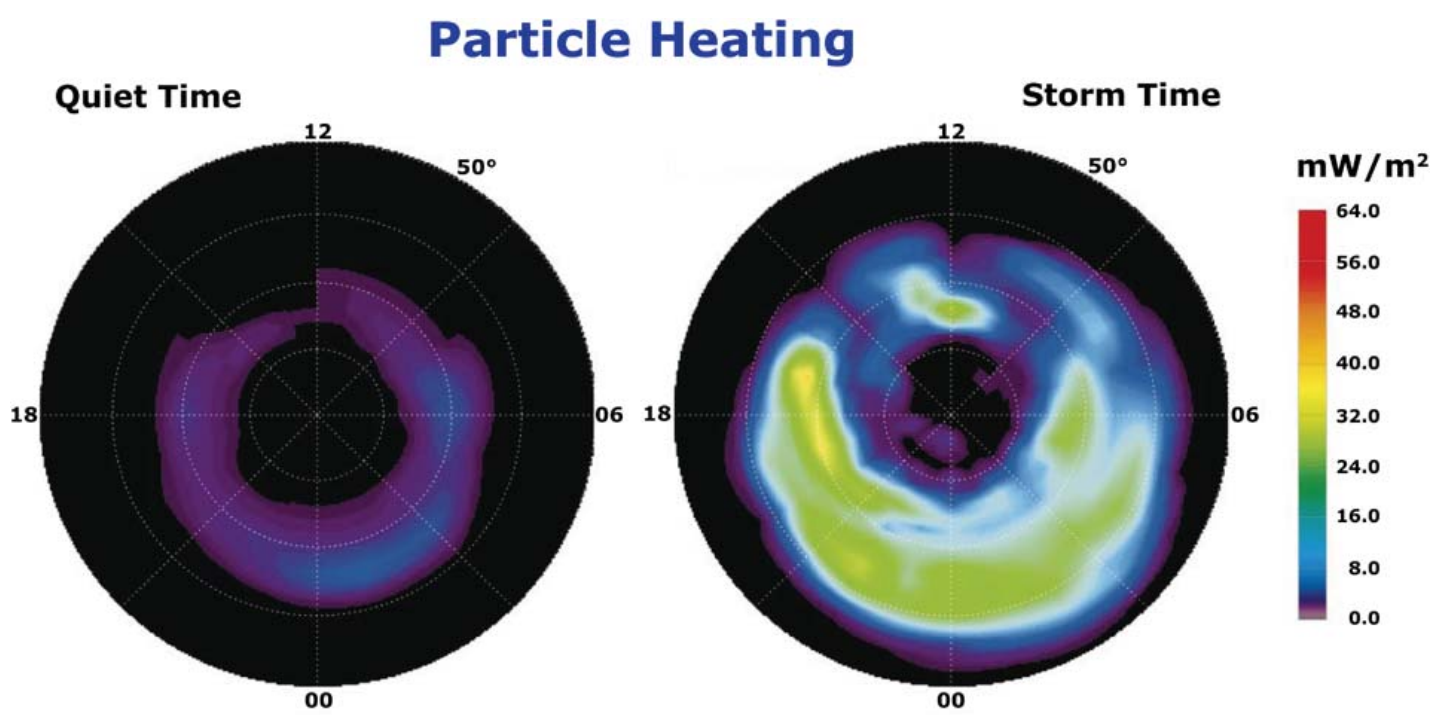

\section{Joule Heating}
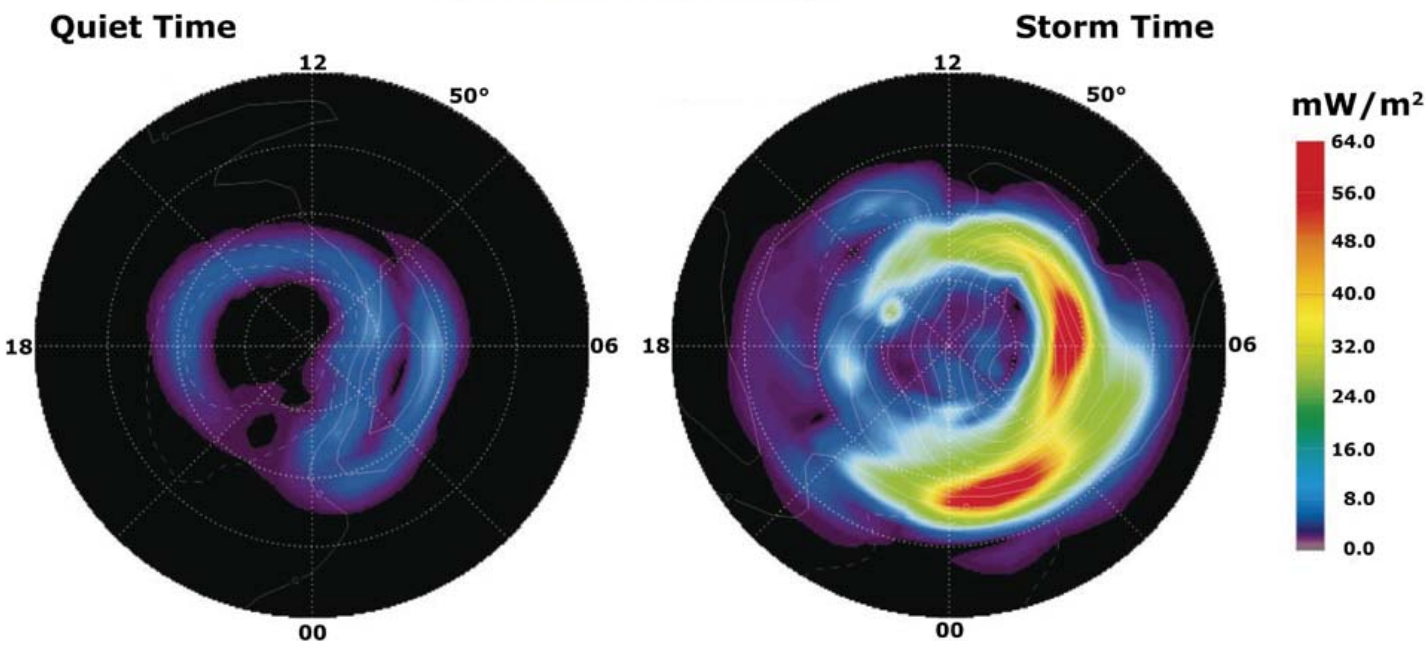

Fig. 62 Distributions in magnetic latitude and local time of the estimated auroral electron energy flux (top), the height-integrated Joule heating rate (bottom), and the electric potential (bottom, contours) above $50^{\circ}$ magnetic latitude in the northern hemisphere for a quiet period on 1997 January 10: 0255 UT (left), and an active period at $1105 \mathrm{UT}$ (right) on the same day. The electric-potential contour interval is $10 \mathrm{kV}$ (Richmond and $\mathrm{Lu} 2000$ )

surges of $100-150 \mathrm{~m} / \mathrm{s}$ which are predominantly on the nightside. Note further that by this time the winds have reached the equator where they will interact with the opposing winds from the other pole, producing an apparent "sloshing" of the winds between hemispheres, as discussed by these authors.

Large neutral winds driven by storms can subsequently lead to changes in the plasma density, electrodynamics, and composition. Indeed, Lu et al. (2009) showed that disturbance neutral winds driven by Joule heating associated with magnetic storms transfer significant energy into the mid and low latitudes in the form of gravity waves. These authors showed that such gravity waves travel with phase speeds close to the local acoustic speed $(\sim 500 \mathrm{~m} / \mathrm{s}$ 
Fig. 63 Model neutral wind variations driven by auroral heating (Fuller-Rowell et al. 2008). The model calculations show the northern component of the wind variation in color, starting at 3 hours after the initiation of an impulse auroral heating event. The vectors (arrows) show the total horizontal component, including the zonal wind component, represented according to the scale at the upper right
Model Wind Variations Driven by Auroral Heating 1500 U.T., $253 \mathrm{~km}$
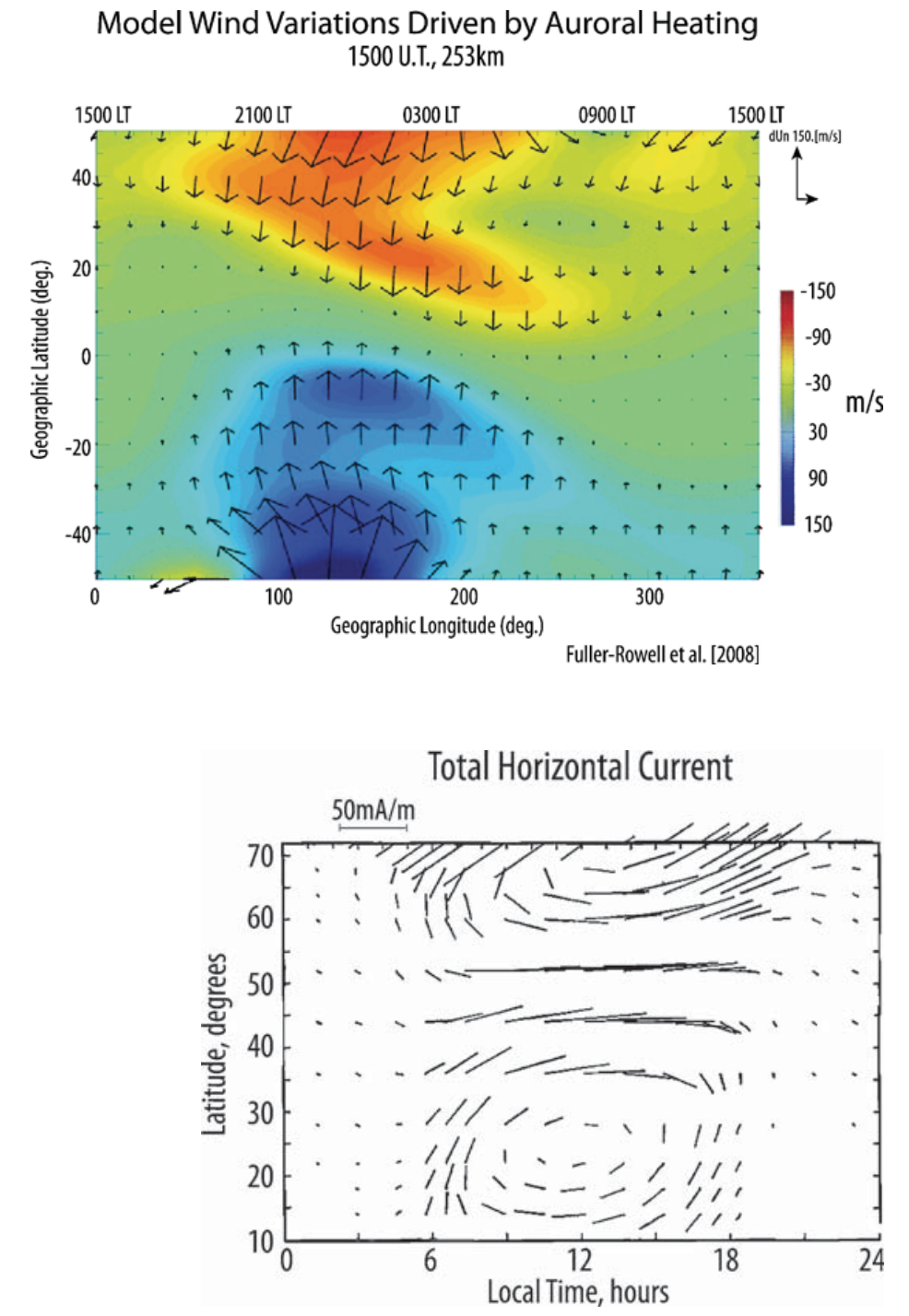

Fig. 64 Model current patterns in the northern hemisphere showing the disturbance dynamo 6 hours after the initiation of a magnetic storm (after Blanc and Richmond 1980)

or higher) and subsequently push plasma up the magnetic field lines, greatly altering the Fregion plasma density profile along their track, as observed by these authors in data gathered at the Millstone Hill and Arecibo incoherent scatter radar observatories.

Disturbance Dynamo. Another consequence of the auroral heating and associated winds directed equatorward from high latitude regions is the creation of new global circulation patterns at lower latitudes, as the equatorward winds develop zonal components at midlatitudes due to Coriolis forces. The resulting circulation creates a system of electric fields and currents known as the disturbance dynamo (Blanc and Richmond 1980). During active conditions, the Sq current patterns discussed earlier (see Fig. 16) actually split into two cells in the upper and lower latitudinal portions of the hemisphere as shown in Fig. 64. Driven by auroral heating at high latitudes, a second cell is introduced with an "anti-Sq" rotation in the upper middle latitudes. Here, strong poleward electric fields driven by strong poleward electric fields that produce a westward $\mathbf{E} \times \mathbf{B}$ drift or eastward currents centered near 45 degree latitude. At the equator, the onset of perturbed east-west electric fields has been shown by Fejer et al. (1983) and Scherliess and Fejer (1997) to be delayed by appropriate time scales, including short (1-12 hrs) and long (22-28 hrs) delays, implying a thermospheric circulation source and supporting the disturbance dynamo theory. Other electrodynamic contributions 
Fig. 65 Images of the earth at $130.4 \mathrm{~nm}$ during the daytime as observed by the VIS camera on the Polar satellite, showing the $\mathrm{O} / \mathrm{N}_{2}$ ratios during a quiet period before a storm on April 16, 2002 (left), and during a magnetic storm on April 19, 2002 (right). The data are provided by J. Sigwarth and J. Kozyra
Polar Satellite -- VIS Earth Camera

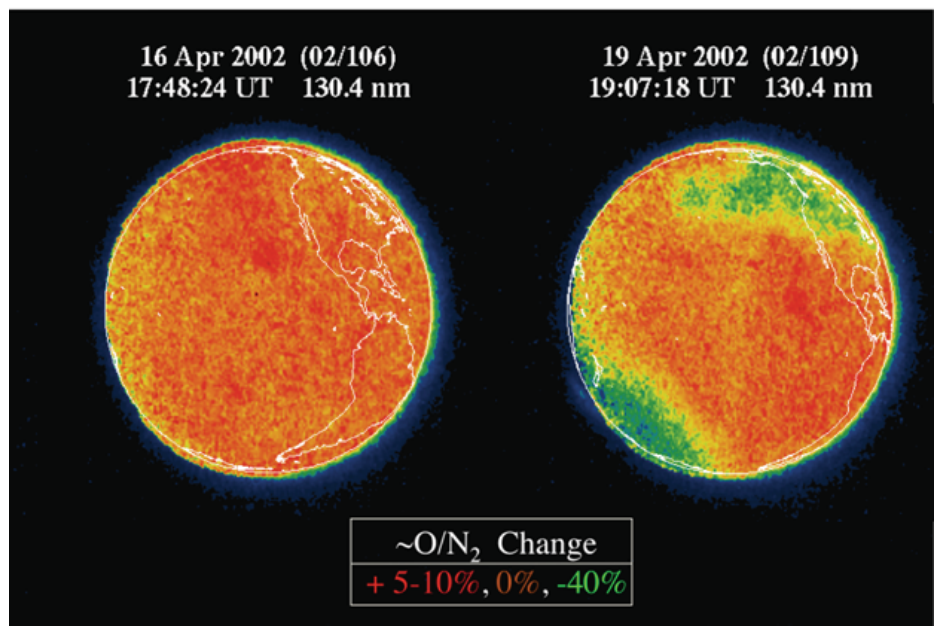

resulting from magnetic storms include those from penetration electric fields and are discussed further on below.

Composition Changes Related to Magnetic Storms. Other major effects of magnetic storms that are quite spectacular are those associated with neutral composition changes that, in turn, affect the plasma density. Variations in the column-integrated $\mathrm{O} / \mathrm{N}_{2}$ ratio observed by the VIS camera on the Polar satellite are presented in Fig. 65 (J. Sigwarth and J. Kozyra, personal communication). The figures show global views of the $130.4 \mathrm{~nm}$ airglow on April 16, 2002 prior to the magnetic storm (left) and on April 19, 2002, during the storm interval (right). Such large-scale composition variations are a well-known consequence of magnetic storms (e.g., Rishbeth et al. 1987; Prölss 1997; Strickland et al. 2001) and are associated with high latitude heating of neutral gases that are then transferred to lower latitudes. The heating causes upwelling of molecules in the air $\left(\mathrm{O}_{2}, \mathrm{~N}_{2}\right)$ and drives changes in the diffusive equilibrium of the neutral species, enriching heavy species and depleting lighter ones. The molecular species become ionized via charge exchange and then undergo rapid loss as recombination is much faster for the molecular ions. Ultimately, the lower conductivity affects the electrical structure of the ionosphere, as well as its coupling with the magnetosphere. Other examples of changes in the column-integrated $\mathrm{O} / \mathrm{N}_{2}$ ratio have been observed in the GUVI instrument on the TIMED satellite, as shown in Fig. 66, and compare well with simulations using the TIMEGCM model (e.g., Meier et al. 2005). A review of current understanding of the variations of $\mathrm{O} / \mathrm{N}_{2}$ ratios during magnetic storms is provided by Crowley and Meier (2008).

Sub-auroral Polarization Streams. Another consequence of magnetic storms is that they create increased pressure gradients at the inner edge of the plasma sheet, as well as strong conductivity gradients in the ionosphere. This combination results in enhanced, polewarddirected electric fields in the pre-midnight sector at the equatorward edge of the auroral oval (Wygant et al. 1998). These electric fields map into the ionosphere and can become quite large, driving strong westward plasma drifts, particularly in the low conductivity region equatorward of the auroral oval in the pre-midnight sector. This phenomenon is known as sub-auroral polarization streams or SAPS, a term proposed by Foster and Burke (2002), that includes similar phenomena known as polarization jets (Galperin et al. 1973) and subauroral ion drifts or SAIDS (Spiro et al. 1979) which have been discussed by numerous 


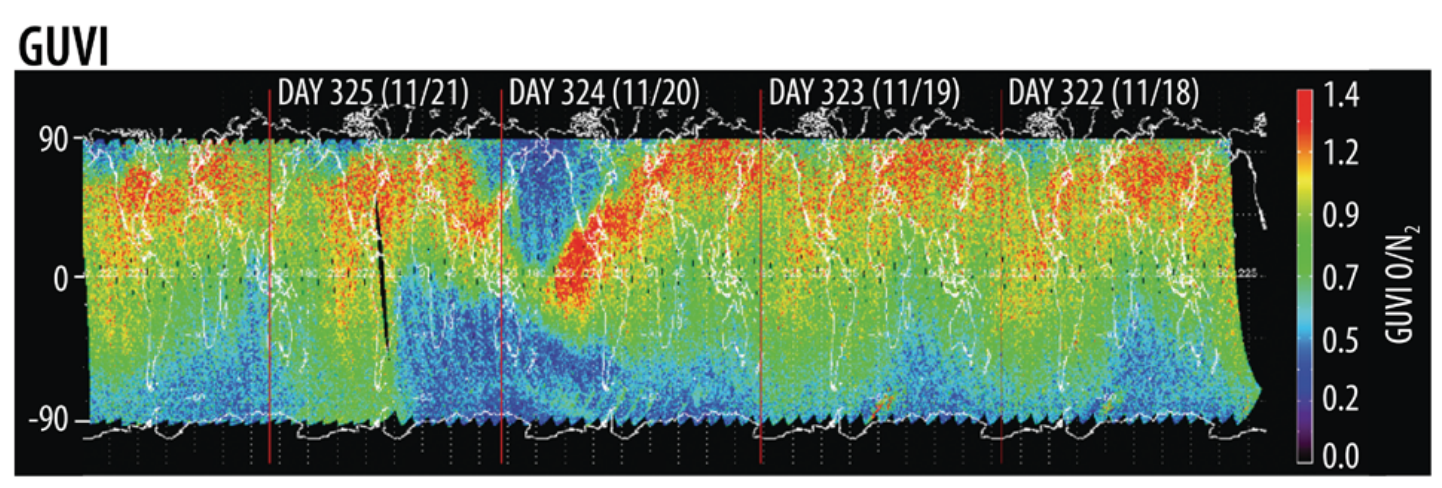

\section{TIME GCM}

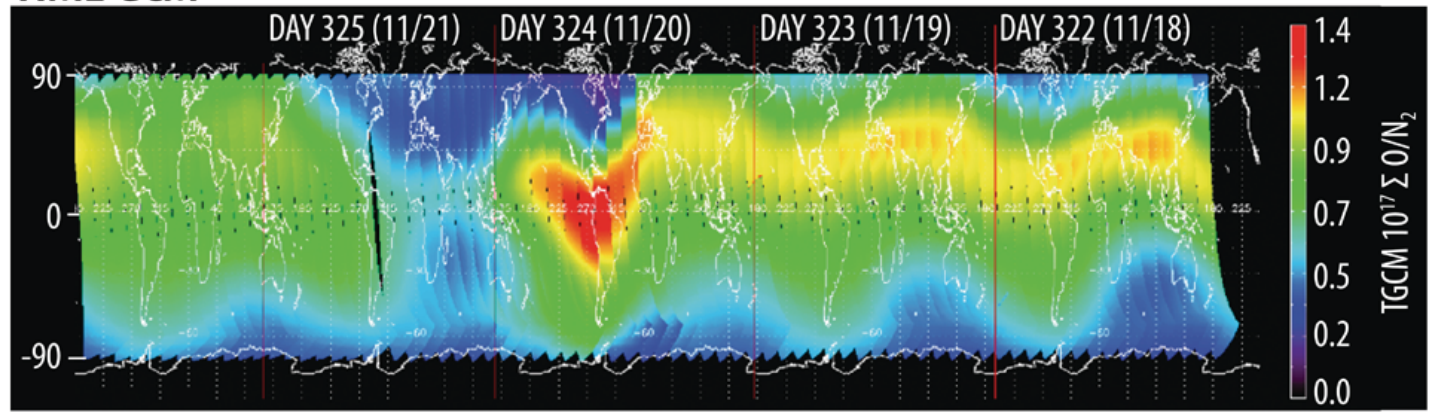

Fig. 66 Observations of $\mathrm{O} / \mathrm{N}_{2}$ ratios as observed by GUVI on the TIMED satellite for five days from November 18-22, 2003 (upper panel), and TIEGCM model calculations of this ratio (Meier et al. 2005). The data are essentially recorded at the same local time, in this case near noon. Note that the observing time (i.e., UT) runs from right to left within each day panel and the panel boundaries are near $0000 \mathrm{UT}$

authors (e.g., Smiddy et al. 1977; Anderson et al. 1991, 1993; Karlsson et al. 1998). Situated equatorward of the auroral precipitation (hence the term, "sub-auroral"), these streams are closely related to where region 2 current are located. In fact, the electric fields are believed to increase within the low conductivity regions to maintain Pedersen current closure of the field-aligned currents. The appearance of enhanced, latitudinally narrow SAIDS has been shown to be related to substorms (e.g., Anderson et al. 1991, 1993; Karlsson et al. 1998). The SAPS phenomenon is an important element in the electrodynamics of magnetic storms (e.g., Burke et al. 2000).

Examples of the enhanced electric fields associated with the sub-auroral polarization streams measured by the Dynamics Explorer-2 satellite are shown in Fig. 67 together with simultaneous measurements of the plasma density (Pfaff et al. 1988). The upper three rows show examples of large amplitude (some greater than $300 \mathrm{mV} / \mathrm{m}$ ) enhancements with narrow latitudinal extent, whereas the bottom row shows examples of broader regions of enhanced electric fields with somewhat lower amplitudes. Examples of polarization streams that are wider and not peaked have also been reported by Foster and Vo (2002). Notice that the enhanced electric fields are often coincident with sharp decreases in the plasma density, though this is not always the case. The enhanced electric fields have been observed in conjugate hemispheres, as indicated by the pairs of data gathered on the same orbit. These observations are not magnetically conjugate as the satellite orbit did not intersect the same magnetic field line as it encountered the SAPS event in the northern and southern hemisphere. However, the data clearly show that the electric fields are enhanced over a wide range of magnetic local time. Simultaneous measurements of this phenomenon at high $(9000 \mathrm{~km})$ and low alti- 


\section{Dynamics Explorer 2}
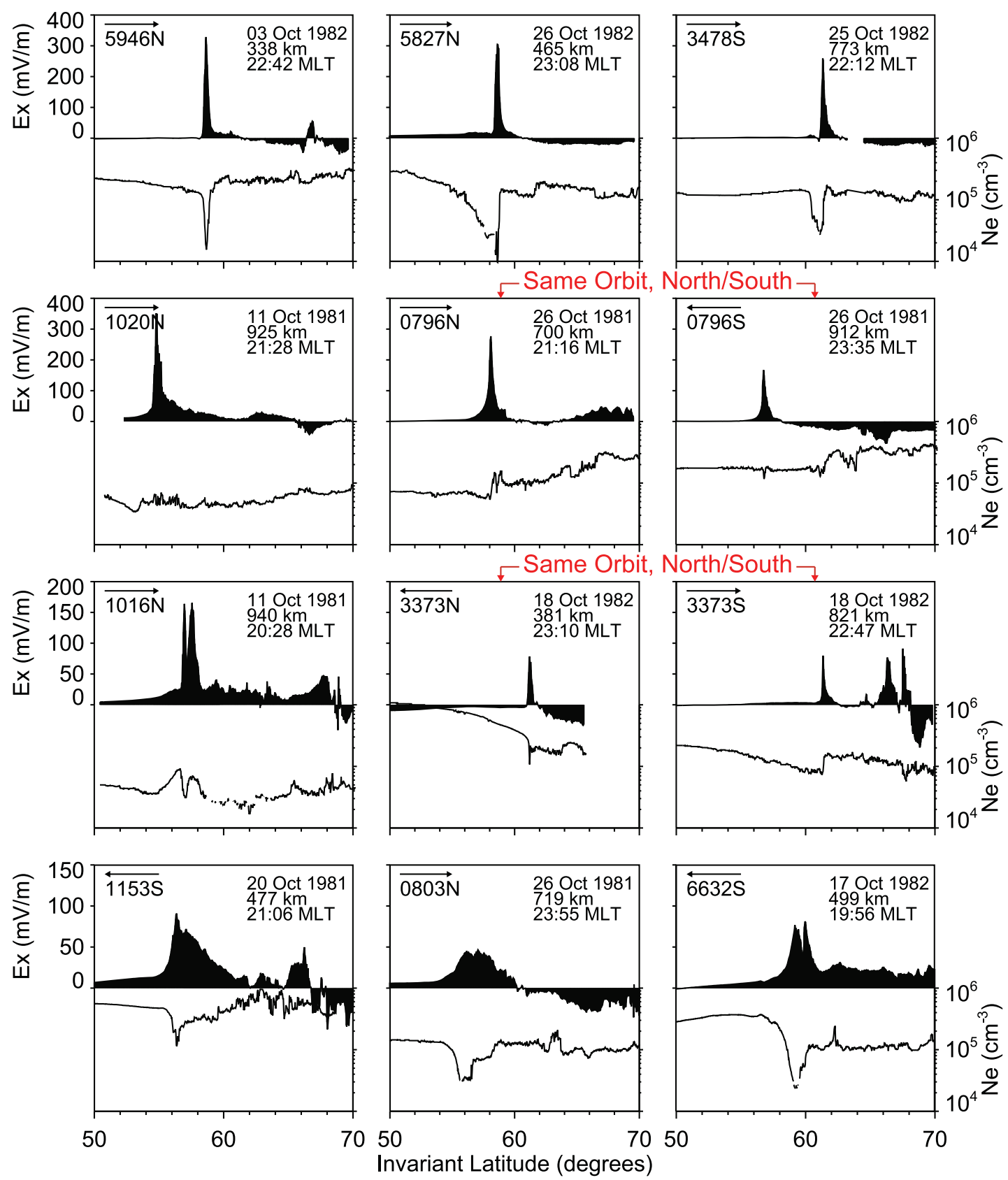

Fig. 67 Examples of large "sub-auroral" electric fields and concurrent plasma density observed by the Dynamics-Explorer-2 satellite. These electric fields are oriented toward the pole producing westward plasma drifts often referred to as SAPS (sub-auroral plasma streams). Two pairs of measurements shown here were recorded along the same orbit, and reveal similar electric fields in conjugate hemispheres

tudes $(840 \mathrm{~km})$ by probes on the Akebono and DMSP satellites, respectively, were reported by Anderson et al. (2001), and support the fact that these fields map between hemispheres along the magnetic field.

SAPS have profound effects on the local ionospheric conductivity and composition, as demonstrated in a series of papers by Anderson et al. (1991, 1993). Pointing out that the rate of the charge exchange reaction (discussed in Sect. 2): 


$$
\mathrm{O}^{+}+\mathrm{N}_{2} \rightarrow \mathrm{NO}^{+}+\mathrm{N}
$$

depends on the 4th power of the velocity differential between the ions and the neutrals (Schunk and Banks 1976), these authors showed that the $\mathrm{NO}^{+}$concentration will increase substantially in regions where strong SAPS are present. Since the recombination rate of $\mathrm{NO}^{+}$is much faster than $\mathrm{O}^{+}$, the plasma number density will decrease further in regions of large electric fields. Anderson et al. (1991) also showed that large vertical ion flows that result from heating via ion-neutral collisions within SAPS will also contribute to the density depletions in the sub-auroral ionosphere.

\subsection{Prompt "Global” Magnetic Storm Effects}

Penetration Electric Fields and Their Effect on Mid-and Low Latitude Plasma Density. The broadening of the auroral oval and the transfer of the Joule heating energy to mid-latitudes is only part of the story of the upper atmosphere's response to magnetic storms. For the largest storms, the entire ionosphere may be subjected to global scale variations such as those driven by prompt penetration electric fields. These have profound effects on the entire ionosphere/upper atmosphere at all latitudes. Such penetration fields are associated with southward turnings of the IMF and are believed to represent interplanetary electric fields that have penetrated the inner magnetosphere to the mid- and low-latitude ionosphere. Examples of such fields are shown in Figs. 68a, 68b in which the zonal electric field measured at the Jicamarca Radio Observatory in Peru (at the magnetic equator) increases in concert with the southward turning of the IMF $B_{z}$ component (Huang et al. 2005). (Note that these authors have included a time shift to allow for the solar wind to travel from the satellite measurement location in the solar wind upstream of the magnetosphere to the ionosphere, which obscures a detailed comparison of the timing of the solar wind events and the exact onset of the ionospheric effects.) Although the details of such penetration fields as well as the shielding efficiency and time constants of the inner magnetosphere are still being debated, these fields appear to have dramatic effects on the distribution of plasma in the low and mid-latitudes.

One of the most important responses of the global ionosphere to large magnetic storms is that the low latitude ionospheric plasma can become depleted within a significant latitudinal extent centered on the magnetic equator, at least for localized longitude regions. Plasma density measurements from consecutive passes of the DMSP satellite in a circular orbit at $840 \mathrm{~km}$ clearly show how the ionospheric plasma "disappeared" from $-20^{\circ}$ to $+20^{\circ}$ (magnetic latitude) during a large magnetic storm (at least for the longitudes sampled), as shown in Fig. 69 (Greenspan et al. 1991). The simultaneously measured upwards plasma drifts were in excess of $200 \mathrm{~m} / \mathrm{s}$ and are shown by these authors to be consistent with plasma being elevated above the satellite altitude. In this manner the phenomenon is similar to the fountain effect (discussed in Sect. 4), driven by sustained, large amplitude east-west zonal electric fields. Discussed in terms of a so-called "super fountain" effect, Heelis (2008) argues that, taking into account neutral winds and other effects, most of the plasma transport under such conditions is poleward (i.e., horizontal). When such density "holes" are created during the daytime due to the large plasma uplift, note that photo-ionization continues at the lower altitudes and the creation of a new F-region proceeds.

An example of large increases in the plasma density in the upper F-region during the daytime occurring within a significant magnetic storm is provided by measurements of total electron content (TEC) above $400 \mathrm{~km}$, as measured by the CHAMP satellite and shown in the lower panel of Fig. 70 (Mannucci et al. 2005). Here, data from three consecutive satellite orbits (spaced $\sim 90$ minutes apart) show how the TEC changes during the storm main 


\section{Solar Wind Observations -- ACE Satellite \\ Ionospheric Electric Fields -- Jicamarca Obs., Peru}

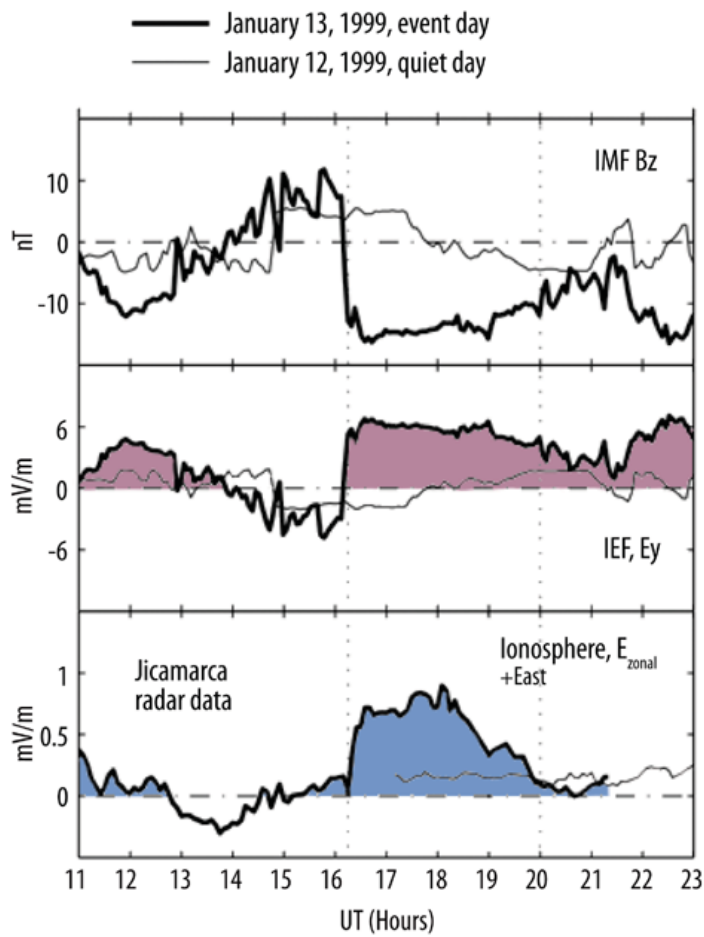

(a)

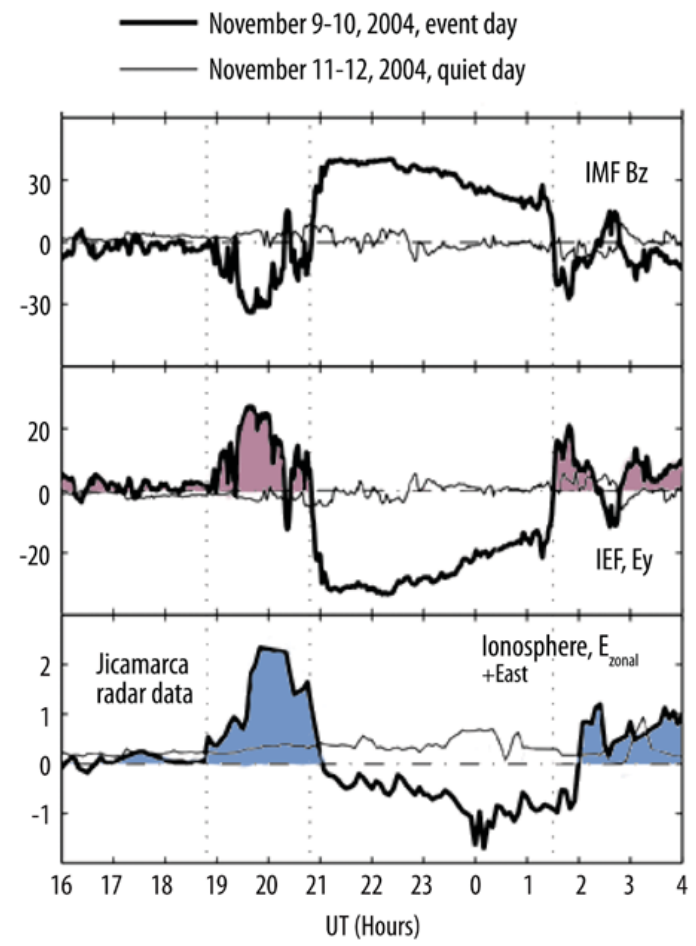

(b)

Fig. 68 Solar wind IMF $B_{z}$ (top) measured by the ACE satellite with associated interplanetary electric field, $E_{y}$ (middle) and the equatorial zonal ionospheric electric field (IEF) $E_{\text {zonal }}$ measured by the Jicamarca radar (bottom). Data from two storm periods are shown, for January 13, 1999 (left) and November 9-10, 2004 (right). Also shown are data recorded during quiet conditions on neighboring days for comparison. The periods shaded in red and blue highlight the interval of positive IEF, corresponding to southward IMF, and enhanced eastward ionospheric electric fields, respectively. Notice the increased scales in the panels on the right, both for the solar wind measurements as well as for the electric fields measured at Jicamarca (adapted from Huang et al. 2005)

phase, indicated by the decrease in Dst, which is plotted in the upper panel. Notice how the maxima of the equatorial anomaly that are normally observed nearer to $\pm 15^{\circ}$ (see blue trace corresponding to 19:00 UT) were "pushed" to values of $\pm 30^{\circ}$ (see red and black traces) yet still retained their approximately symmetric, winged shape. These data were gathered near $1300 \mathrm{hrs} \mathrm{SLT}$ at low and middle latitude (A. Mannucci, personal communication, 2012) and show prompt dayside total electron content increases of $\sim 900 \%$ above $400 \mathrm{~km}$, suggesting that the normal, daytime ionosphere at lower altitudes had risen above the CHAMP satellite altitude. These authors also invoke prompt penetrating electric fields which create plasma uplift with associated diffusion along the magnetic field lines to higher latitudes to explain both the large TEC values and the observed, symmetric latitudinal spreading. A different interpretation for the storm-time density enhancements on the dayside is that due to high latitude convection electric fields expanded equatorward, as discussed by Heelis et al. (2009a, 2009b) and Heelis and Mohapatra (2009).

Examples of Low and Mid-Latitude Irregularities Associated with Magnetic Storms. To demonstrate further how the ionospheric storm response appears to be initiated at the equator 
Fig. 69 Log of total ion density in $\mathrm{cm}^{-3}$ (upper panels, light orange) measured by the DMSP spacecraft plotted as a function of magnetic latitude for five successive passes, each near 2130 LT, during the magnetic storm of March 13-14, 1989. The lower panels show measurements of the vertical ion drifts in which the positive values are shaded blue. The labels to the right of each plot give the universal time and geographic latitude and longitude at which the spacecraft crossed the magnetic equator (adapted from Greenspan et al. 1991) (a)

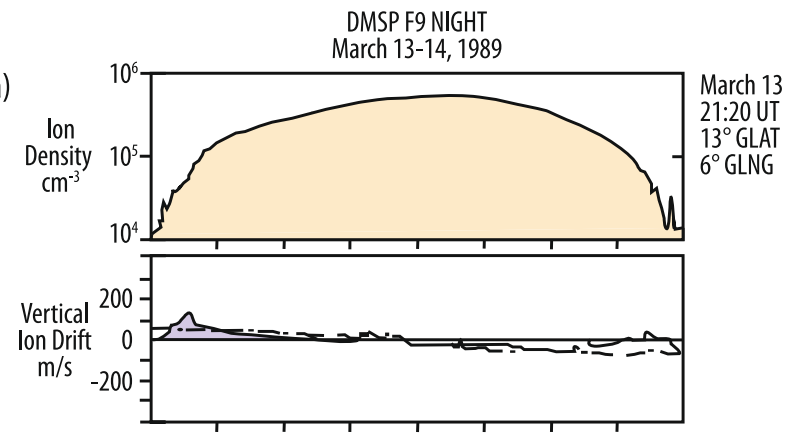

(b)

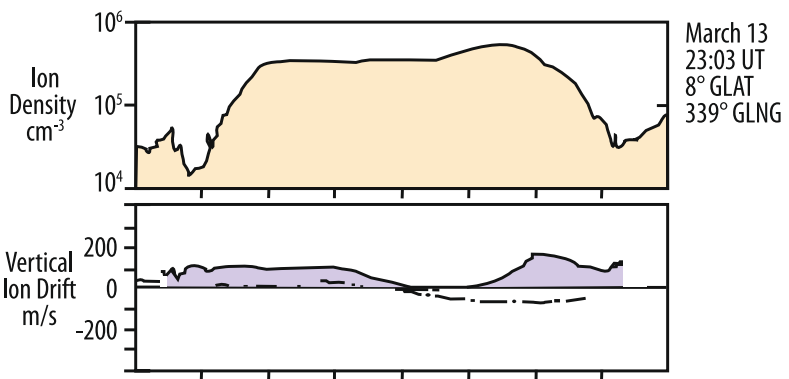

(c)

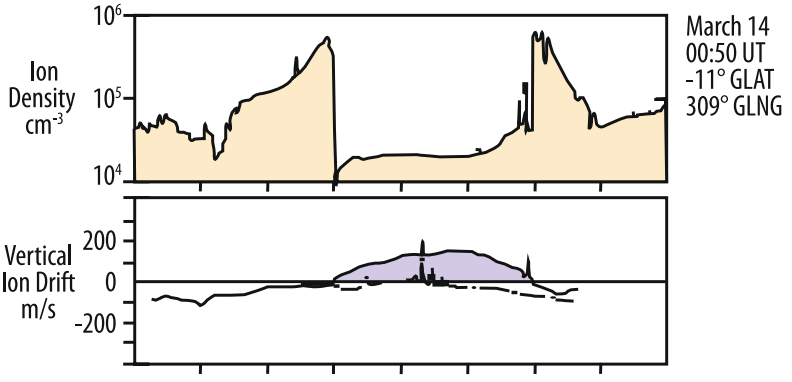

(d)

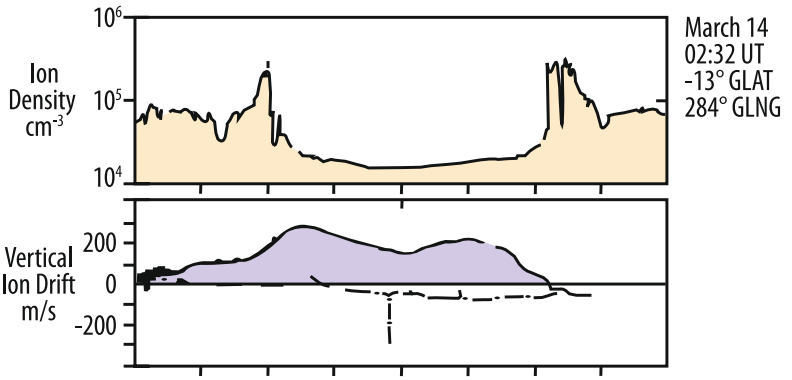

(e)

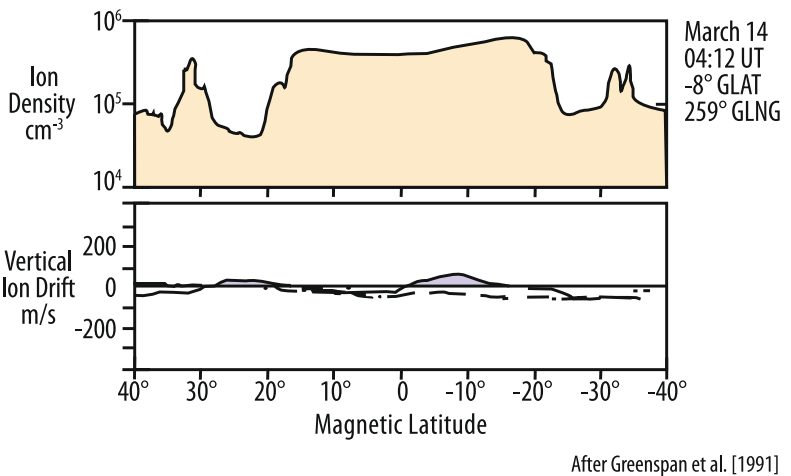




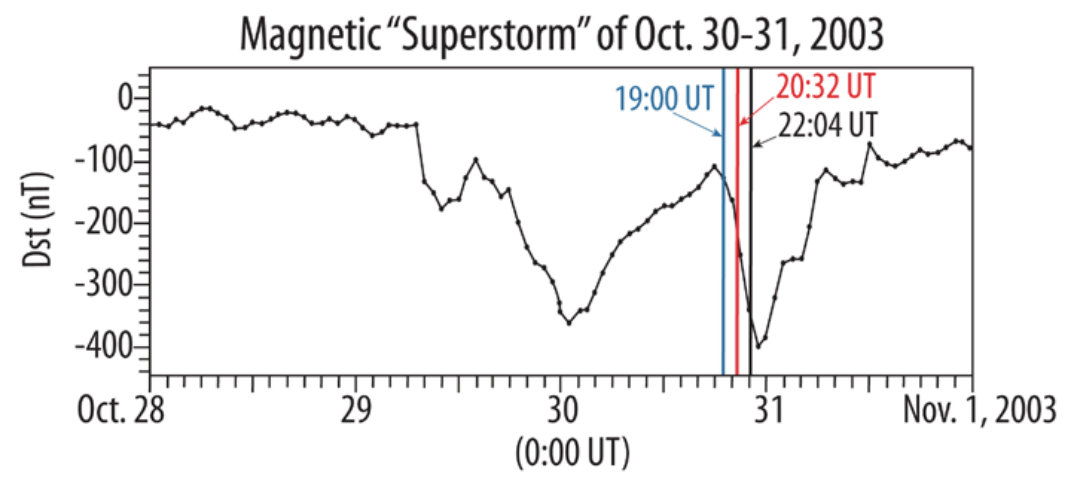

\section{Dayside TEC Above CHAMP Satellite at $400 \mathrm{~km}$}

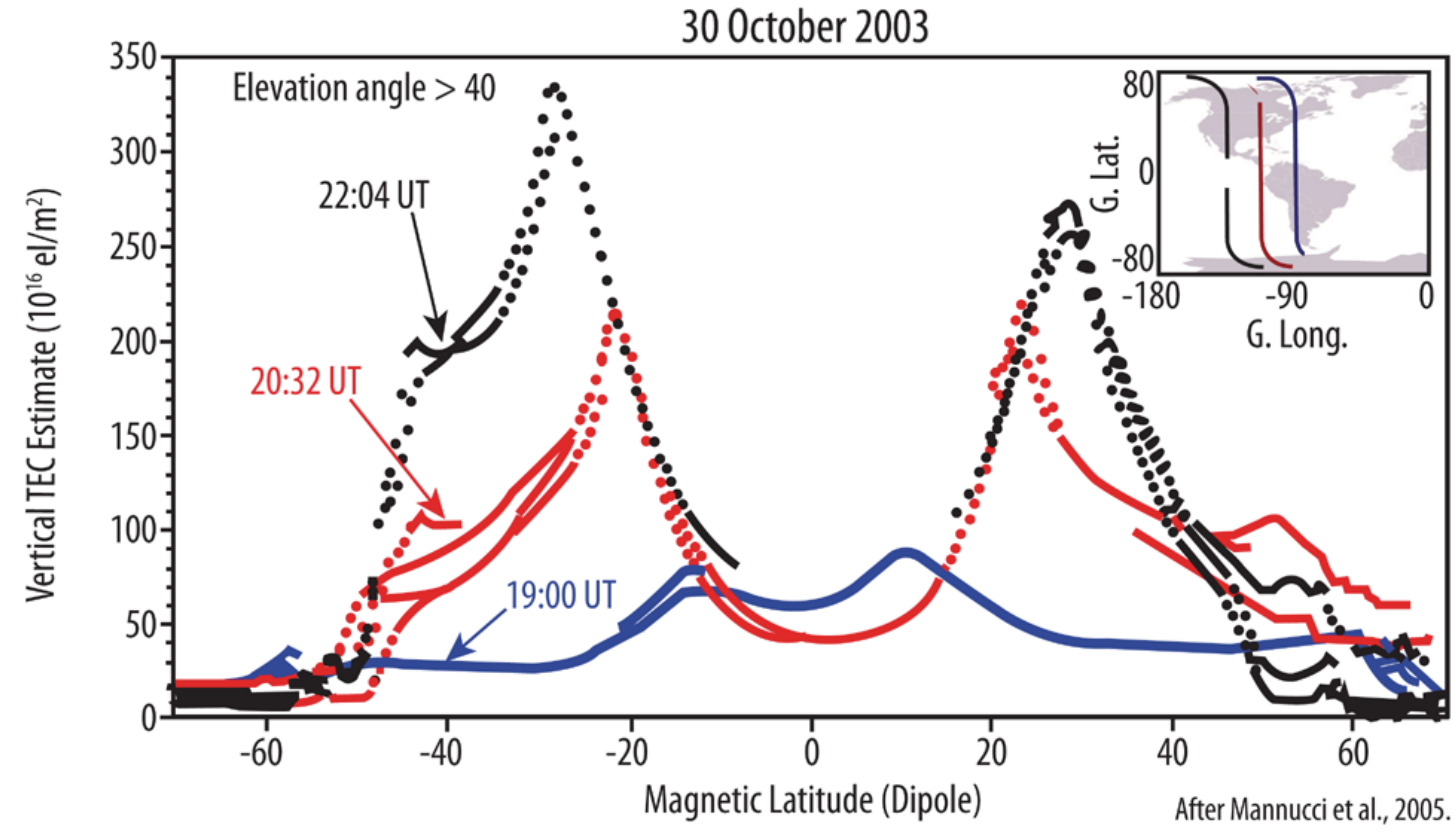

Fig. 70 The integrated electron content (corresponding to total electron content or TEC) as measured by the GPS instrument on the CHAMP satellite for altitudes above the CHAMP altitude of $400 \mathrm{~km}$ (lower panel). Data are shown for three orbits plotted as a function of magnetic latitude which correspond to times just prior to (blue) and after (red and black) the onset of a magnetic storm on October 30, 2003, as shown in the plot of Dst in the upper panel. The locations of the CHAMP orbits are shown in the upper right corner of the lower panel. The local times of these orbits range from 1230-1330 LT for latitudes within \pm 60 degrees. Points missing near the anomaly trough are due to the elevation angle cut-off (adapted from Mannucci et al. 2005)

and then move polewards simultaneously in both hemispheres, at least during the evening, we show DEMETER satellite data in Fig. 71. Consecutive orbits of plasma density and electric field irregularities are presented for two different magnetic storms, as shown in the Dst data in the upper panels (Pfaff et al. 2008). These data were gathered along circular orbits at $710 \mathrm{~km}$ in the evening ( $\sim 22.5 \mathrm{LT})$ sector. At the storm commencement the first indication of both enhanced plasma density and associated irregularities at $710 \mathrm{~km}$ occurred at the equator, and then proceeded towards the poles in both hemispheres as the storm progressed. The irregularities associated with the inter-hemispheric, unstable flux tubes continue to rise in altitude and latitude, symmetrically in both hemispheres and are encountered by the consecutive DEMETER orbits at successively higher latitudes. These data reinforce the hypothesis that the storm response at low and mid-latitudes is initiated at the equator. At the same time, 


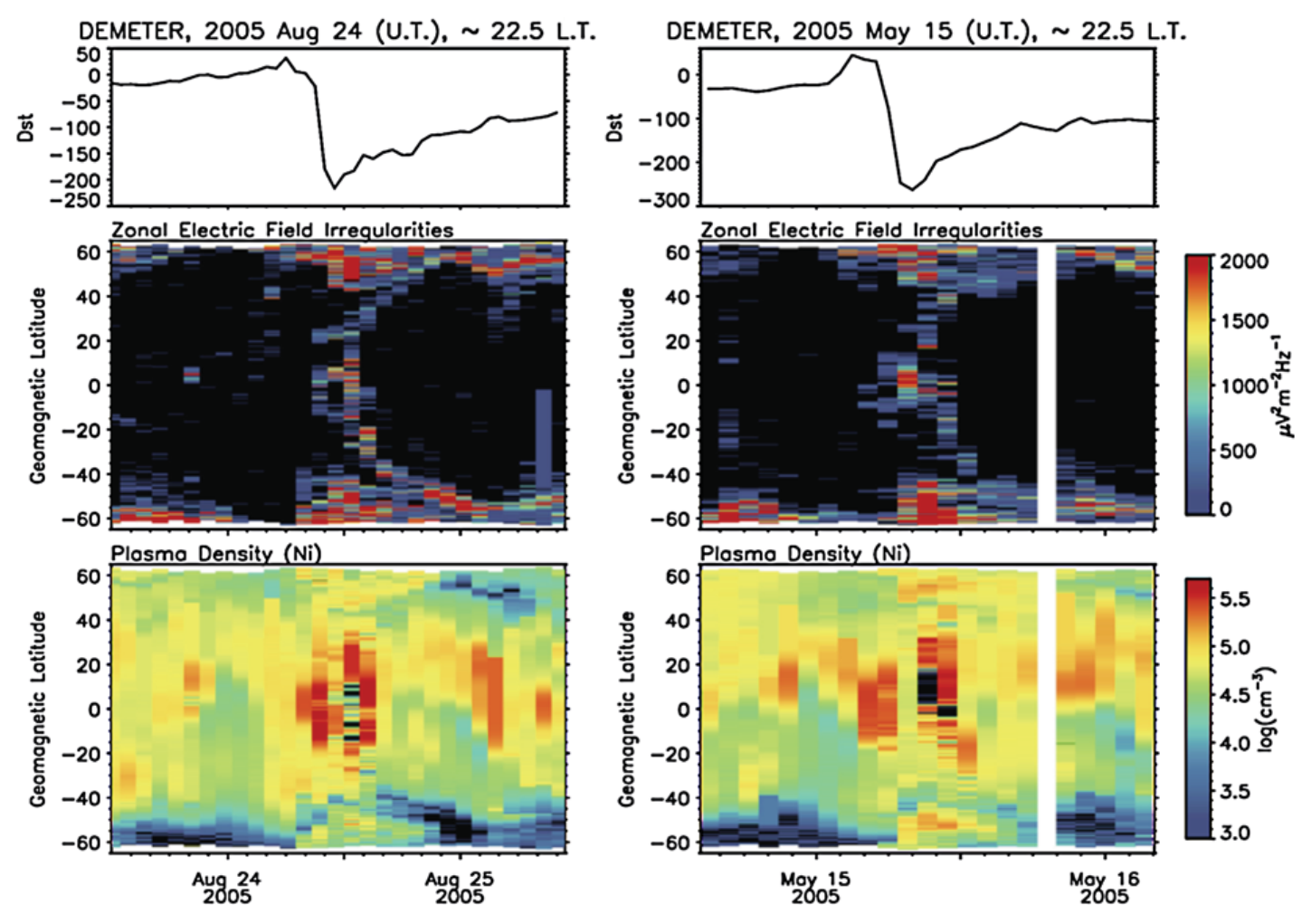

Fig. 71 DEMETER satellite data (near 22:30 LT) for the magnetic storm periods of August 24, 2005 (a) and May 15, 2005 (b). The top panels show the Dst estimates based on ground-based magnetic field measurements. The middle and lower panels show the DEMETER integrated electric field irregularity power from 10-100 Hz and the plasma number density along each orbit. The satellite data have been converted to color scales and mapped to bins of magnetic latitude between -65 and +65 degrees (Pfaff et al. 2008)

the polar cap high latitude ionosphere widens and expands equatorward, as evidenced in the lower panels in Fig. 71 where the plasma density decrease or trough near 60 degrees magnetic latitude prior to the storms expands equatorward towards \pm 40 degrees magnetic latitude in both the northern and southern hemispheres.

Ground-based observatories at mid latitudes have also observed ionospheric irregularities associated with magnetic storms that provide important clues concerning whether unstable flux tubes might be set up between hemispheres, as suggested by the DEMETER data above. Two examples of radar measurements gathered in Puerto Rico during magnetic storms are shown in Fig. 72. The upper right-hand panel shows data gathered with a VHF coherent scatter radar looking northward from Puerto Rico (figure courtesy E. Kudeki; see Swartz et al. 2000) and shows irregularities emerging from what is believed to be descending F-region ledge, similar to those associated with low latitude spread-F. The lower panel shows Arecibo incoherent scatter measurements during a similar magnetic storm (Kelley et al. 2002). Although the fixed radar beam cannot distinguish temporal and spatial variations that may be at work during this event, the data suggest the presence of depleted flux tubes that emerge from the F-region ledge and which may be expected to give rise to electrostatic irregularities. Other measurements (e.g., TEC and airglow depletions) during these and similar magneticallydisturbed events at mid latitudes have been reported by other researchers (e.g., Makela et al. 2000; Kelley et al. 2000; and Bust et al. 2000) and demonstrate that the midlatitude ionosphere becomes highly structured at night during magnetically disturbed conditions. 

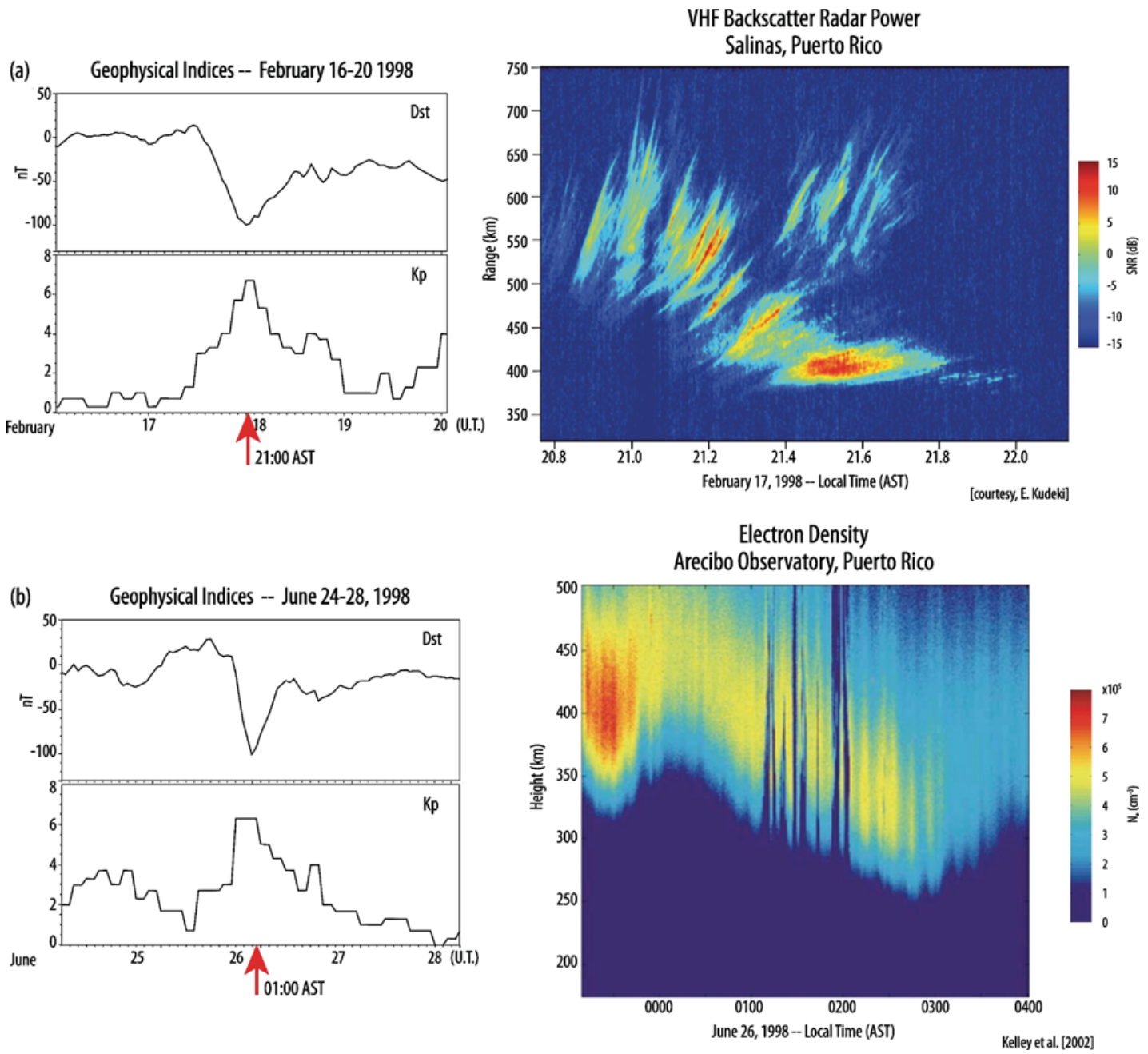

Fig. 72 Measurements by ground-based instruments in Puerto Rico of plasma density structures associated with two modest magnetic storms, whose Dst and Kp signatures are shown on the left hand side. The panel on the upper right shows $50 \mathrm{MHz}$ backscatter radar measurements that resemble spread-F scatter that were coincident with the period of largest negative Dst (figure courtesy E. Kudeki; See also Swartz et al. 2000). The panel on the lower right shows plasma density measurements from the incoherent scatter radar at Arecibo that were also coincident with the period of the largest negative Dst (Kelley et al. 2002). The large plasma depletion "chimneys" that emerge from the lower edge of the F-region between 0100 and 0200 LT resemble spread-F depletions more commonly observed at lower latitudes

The panels showing the Dst data on the left hand side of Fig. 72 indicate that in each case, the radar irregularities and depletions were observed at the peak times of their respective storms. The fact that the radars in each case were situated on the nightside during each of these two storm events, in which the main phase lasted less than 12 hours, undoubtedly helped enable the observations of these particular storm signatures. These mid-latitude observations of F-region irregularities and depletions resemble spread-F processes known to occur at low latitudes at night.

Storm-Enhanced Density Structures. A final example of significant effects on the nearearth plasma from magnetic storms is planetary-scale plasma density structures that appear to break off from the enhanced mid-latitude plasma that accumulates during a large amplitude magnetic storm and then is transported poleward, as shown in Fig. 73 (figure courtesy of A. Coster). These plumes are referred to as storm enhanced density (SED) erosion plumes (see Foster et al. 2005, and references therein) and are evi- 
Fig. 73 Storm-enhanced plasma density (SED) signatures in total electron content (TEC) observed on November 20, 2003. These are believed to be connected to plasmasphere erosion and driven by sub-auroral electric fields from the inner magnetosphere. Strong plasma density gradients are observed by a network of ground-based GPS observatories. Figure courtesy of A. Coster

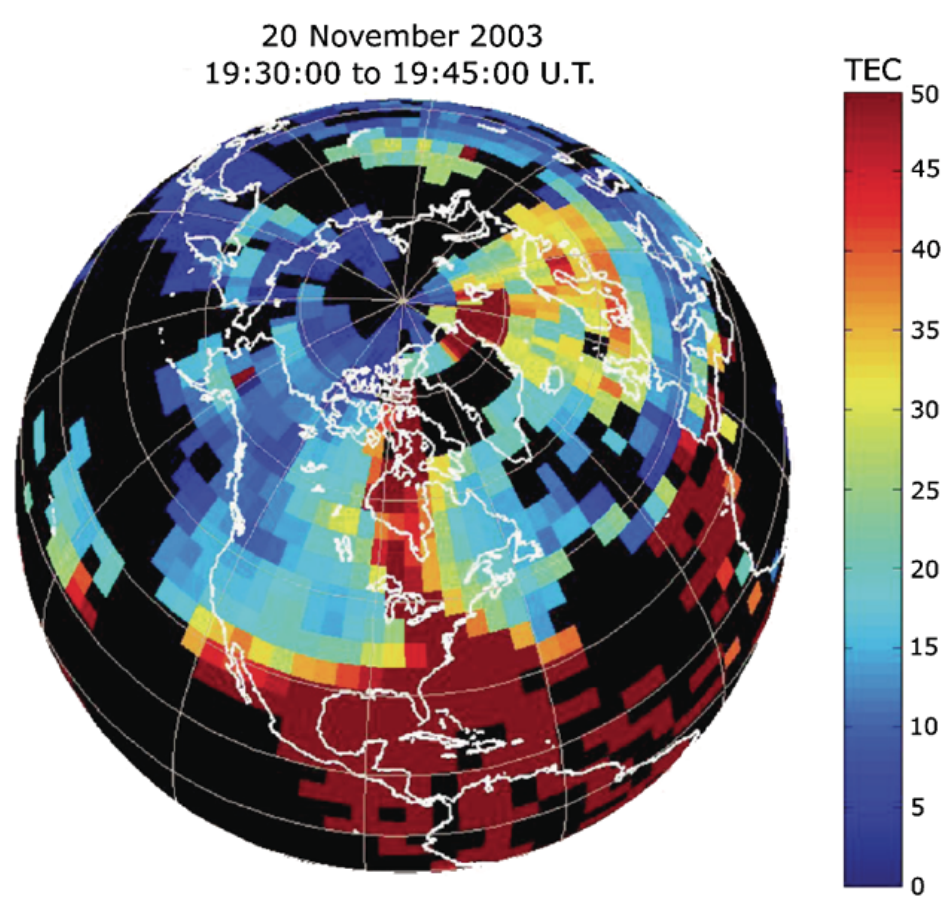

dent in widely spaced GPS measurements of total electron content. Foster et al. (2005) reported that the plume shown in the figure is carried through the cusp during the main phase of the magnetic storm of November 20, 2003. In such a scenario, the enhanced plasma density is driven upwards and poleward by the prompt penetration electric fields, then towards the dayside by storm-related SAPS, and finally by dayside, antisunward convection to form the plume across the polar cap at higher latitudes. Such plumes which arrive at the high latitudes may enhance ion outflow into the magnetosphere.

\section{Summary}

In this paper, we have reviewed the origins of the near-earth plasma created by photoionization due to solar EUV radiation, primarily at mid and low latitudes, and by impact ionization from precipitating energetic particles at higher latitudes. Not only is the nearearth plasma created directly from the earth's extended atmosphere, but the ionization that is formed remains coupled to the neutral gases and their dynamics. Clearly, to fully understand the near-earth space environment, we must consider both the neutral and ionized gases as an ensemble, forming one envelope that surrounds our planet.

We have examined many of the numerous processes and dynamics inherent in the coupled ion-neutral gas at low and mid latitudes. Throughout this region, the ionized gas component is largely controlled by the neutral dynamics. However, as we have shown, small changes between the ion and electron distributions, primarily set up as a result of collisions with the neutral particles, are sufficient to generate global systems of currents and electric fields. These currents map along the magnetic field lines to other regions of Geospace where their effects may be profound, for example near the magnetic equator just after sunset. Furthermore, the fact that the ionosphere/upper atmosphere is strongly influenced from forcing from below-i.e., from tides, gravity waves, and thunderstorm activity originating in the troposphere-underscores the controlling role of the atmosphere on the behavior of the ionized gases, particularly at mid and low latitudes. 
We have also examined the dynamics and processes inherent in the coupled ion-neutral gas at high latitudes. We have shown how strong electric fields and currents originating in the magnetosphere often dominate the dynamics, "stirring the pot" of the polar regions with complex convection and field-aligned current patterns that together yield a self-consistent electrodynamic picture. As the magnetospheric electric fields drive neutral winds via ionneutral collisions, the neutral atmosphere is shown to play a key role in the conversion of magnetospheric electromagnetic energy to mechanical energy in the high latitude upper atmosphere. Furthermore, the neutral atmosphere also plays a critical role in determining how the magnetospheric energy is dissipated, primarily through Joule heating which essentially results from friction between the differential motion of the neutral and ionized gases. Indeed, the response of the neutral gases includes large horizontal and vertical flows that are not confined by the magnetic field and which can subsequently convey the magnetospheric energy to locations far beyond where it was initially deposited. The neutral atmosphere also affects the closure, in the lower ionosphere, of magnetospheric field-aligned currents via Pedersen currents. Finally, the local conductivity in the ionosphere, which is also affected by the neutral atmosphere, has been shown to have a direct effect on the creation of electrostatic structures at higher altitudes that accelerate the energetic particles that ultimately precipitate to form the discrete aurora.

We have also examined many of the fundamental physical processes inherent in the nearearth plasma that result from magnetic storms, such as the effects of increased energy deposition at high latitudes, and have shown how such energy is then distributed away from the polar regions. We also showed evidence that the global ionosphere is subjected to large scale variations when the earth is impacted by magnetic storms of appreciable magnitude. Such effects include substantial changes in the ionospheric density, which may be attributable to prompt penetration electric fields which have been observed at the magnetic equator near the onset of magnetic storms. These observations demonstrate the global and rapid response of the nearearth plasma to solar wind forcing associated with significant magnetic storms.

Progress in our understanding of the ionosphere/thermosphere system requires adopting a systems approach with respect to interpreting the large scale dynamics associated with the energy and momentum exchanges within this gaseous envelope as it adjusts internally and responds to input from both the troposphere below and the magnetosphere above. Understanding such a self-consistent electrodynamic and hydrodynamic response to these drivers is a critical, long-standing objective which is at the forefront of future research in this field.

One area that is highly relevant to future progress in our understanding is that of the tremendous promise within the modeling community of incorporating both physics and observations in computational models of the upper atmosphere. Examples of such models that include first principles of ionosphere/thermosphere coupling are the ThermosphereIonosphere-Mesosphere-Electrodynamics General Circulation Model (TIMEGCM) (Roble et al. 1988) and the Coupled Thermosphere-Ionosphere-Plasmasphere-Electrodynamics (CTIPe) model (Fuller-Rowell et al. 1996). Furthermore, the Whole Atmosphere Model (WAM) (Fuller-Rowell et al. 2010) is an extension of the US National Weather Service Global Forecast System and includes the impact of tides, planetary waves, and gravity waves. Finally, the Assimilative Mapping of Ionospheric Electrodynamics (AMIE) model (Richmond et al. 1992) includes the most accurate high latitude specification, which is important for understanding the high latitude forcing.

While the models mentioned above rely on both physical understanding and observations, in some areas the models are considerably "data starved". For example, very few simultaneous measurements of the dynamics of neutral and ionized gases at the lower altitudes exist. Although targeted research rockets, satellites, and ground-based measurements 
are essential for advancing our understanding, the systems approach requires simultaneous measurements using a number of observational techniques, including space-based in situ and imaging experiments as well as arrays of ground-based radars and other instruments. The space-based direct measurements show particular promise when organized as "constellation" missions, particularly in configurations where data may be gathered simultaneously at multiple local times and longitudes. Such platforms promise to reveal how the neutral and ionized dynamics are organized, as well as show how energy and momentum is distributed, and re-distributed, globally. For example, such measurements would reveal the two-dimensional coupling of neutral and plasma gases and their dynamics across the entire high latitude ionosphere, show the global consequences of propagating tides, and demonstrate how magnetic storms promptly effect the entire ionosphere/thermosphere system as a function of latitude, local time, and longitude.

Finally, we emphasize that to be most effective, such measurement platforms need to journey where the most important physical processes are actually taking place. In other words, to examine how the ionized and neutral gases exchange energy and momentum, it is important that the instruments sample altitudes where these interactions are most profound. For example, simultaneous measurements of ions and neutrals below $400 \mathrm{~km}$ are essential to understand the coupled behavior of these gases. Focused missions that furthermore explore well below $300 \mathrm{~km}$ are expected to reveal important fundamental physical processes involving the ion-neutral gases where the coupling is maximized and where our knowledge of the resulting gas dynamics is rudimentary, at best.

Ultimately, the behavior of the Earth's ionized and neutral gases depends on non-linear coupling between complex, highly varying external sources. These external sources include the variable EUV radiation from the sun as well as intricate energy and momentum exchanges with the troposphere/stratosphere/mesosphere below and the magnetosphere and solar wind above. It is precisely because these coupled regions are exceedingly rich in physical processes that the near-earth plasma is one of the most exciting, and important, natural environments in which to focus scientific research and resources. The new knowledge promised from such future research is essential, not only for understanding the Earth, but also for understanding the upper atmospheres of other planets, both in our solar system and beyond.

Acknowledgements I am indebted to Dr. J. Klenzing for calculations of the model ionosphere parameters provided in this article. I thank Drs. L. Kepko, J. Grebowsky, J. Clemmons, R. Walterscheid, D. Rowland, and H. Freudenreich for very useful comments on the entire manuscript. I thank Drs. G. Le, P. Anderson, T. Fuller-Rowell, and J. Thayer, for valuable comments on individual sections or sub-sections. I thank Ms. H. Joliffe and Dr. S. Martin for providing many of the figures shown here, as well as other aspects of the manuscript preparation.

\section{References}

T. Abe, B.A. Whalen, A.W. Yau, R.E. Horita, S. Watanabe, E. Sagawa, EXOS-D (Akebono) suprathermal mass spectrometer observations of the polar wind. J. Geophys. Res. 98, 11191-11203 (1993)

T.L. Aggson, Probe measurements of electric fields in space, in Proc. NATO Adv. Study Inst (1969), p. 305

T.L. Aggson, N.C. Maynard, W.B. Hanson, J.L. Saba, Electric-field observations of equatorial bubbles. J. Geophys. Res. 97, 2997-3009 (1992)

P.C. Anderson, R.A. Heelis, W.B. Hanson, The ionospheric signatures of rapid subauroral ion drifts. J. Geophys. Res. 96, 5785-5792 (1991)

P.C. Anderson, W.B. Hanson, R.A. Heelis, J.D. Craven, D.N. Baker, L.A. Frank, A proposed production model of rapid subauroral ion drifts and their relationship to substorm evolution. J. Geophys. Res. 98, 6069-6078 (1993). doi:10.1029/92JA01975 
P.C. Anderson, D.L. Carpenter, K. Tsuruda, T. Mukai, F.J. Rich, Multi-satellite observations of rapid subauroral ion drifts (SAID). J. Geophys. Res. 106, 29585-29600 (2001)

M. André, A. Yau, Theories and observations of ion energization and outflow in the high latitude magnetosphere. Space Sci. Rev. 80, 27-48 (1997)

E.V. Appleton, Two anomalies in the ionosphere. Nature 157, 691 (1946)

P.M. Banks, G. Kockarts, Aeronomy, Part A (Academic Press, New York, 1973)

A. Berthelier, J.-C. Cerisier, J.-J. Berthelier, J.-M. Bosqued, R.A. Kovrazkhin, The electrodynamic signature of short scale field aligned currents, and associated turbulence in the cusp and dayside auroral zone, in Electromagnetic Coupling in the Polar Clefts and Caps, ed. by P.E. Sandholt, A. Egeland (Kluwer Academic, Dordrecht, 1989)

D. Bilitza, B.W. Reinisch, International reference ionosphere 2007: improvements and new parameters. Adv. Space Res. 43, 599-609 (2008). doi:10.1016/j.asr.2007.07.048

D. Bilitza, L.-A. McKinnel, B. Reinisch, T. Fuller-Rowell, International reference, ionosphere today and in the future. J. Geod. 85, 909-920 (2011). doi:10.1007/s00190-010-0427-x

K. Birkeland, On the cause of magnetic storms. C. R. Hebd. Séances l'Acad. Sci. 147, 539-543 (1908)

M. Blanc, A.D. Richmond, The ionospheric disturbance dynamo. J. Geophys. Res. 85, 1669-1686 (1980)

R. Boström, Electrodynamics of the ionosphere, in Cosmical Geophysics, ed. by A. Egeland, O. Holter, A. Omholt (Universitetsforlaget, Oslo, 1973)

D.G. Brinkman, R.L. Walterscheid, L.R. Lyons, D.C. Kayser, A.B. Christensen, E region neutral winds in the postmidnight diffuse aurora during the Atmospheric Response in Aurora 1 rocket campaign. J. Geophys. Res. 100, 17,309-17,320 (1995)

J.E. Buchau, J. Weber, D.N. Anderson, H.C. Carlson Jr., J.G. Moore, B.W. Reinisch, R.C. Livingston, Ionospheric structures in the polar cap: their origin and relation to $250 \mathrm{MHz}$ scintillation. Radio Sci. 20, 325 (1985)

O. Buneman, Excitation of field aligned sound waves by electron streams. Phys. Rev. Lett. 10, 285-287 (1963)

W.J. Burke, T.L. Aggson, N.C. Maynard, W.R. Hoegy, R.A. Hoffman, R.M. Candy, C. Liebrecht, E. Rodgers, Effects of a lightning discharge detected by the DE 2 satellite over Hurricane Debbie. J. Geophys. Res. 97, 6359-6367 (1992). doi:10.1029/92JA00305

W.J. Burke, A.G. Rubin, N.C. Maynard, L.C. Gentile, P.J. Sultan, F.J. Rich, O. de La Beaujardière, C.Y. Huang, G.R. Wilson, Ionospheric disturbances observed by DMSP at middle to low latitudes during the magnetic storm of June 4-6, 1991. J. Geophys. Res. 105, 18391-18405 (2000)

G.S. Bust, D. Coco, J.J. Makela, Combined ionospheric campaign. 1. Ionospheric tomography and GPS total electron count (TEC) depletions. Geophys. Res. Lett. 27, 2849-2852 (2000)

C.W. Carlson, J.P. McFadden, R.E. Ergun, M. Temerin, W. Peria, F.S. Mozer, D.M. Klumpar, E.G. Shelley, W.K. Peterson, E. Moebius, R. Elphic, R. Strangeway, C. Cattell, R. Pfaff, FAST observations in the downward auroral current region: energetic upgoing electron beams, parallel potential drops, and ion heating. Geophys. Res. Lett. 25, 2017-2020 (1998)

Z. Ceplecha, Influx of interplanetary bodies onto earth. Astron. Astrophys. 263, 361-366 (1992)

E. Chapin, E. Kudeki, Plasma-wave excitation on meteor trails in the equatorial electrojet. Geophys. Res. Lett. 21, 2433-2436 (1994)

S. Chapman, The absorption and dissociative or ionizing effect of monochromatic radiation in an atmosphere on a rotating earth. Proc. Phys. Soc. London 43, 26-45 (1931)

C.R. Chappell, J.L. Green, J.F.E. Johnson, J.H. Waite Jr., Pitch angle variations in magnetospheric thermal plasma - initial observations from Dynamics Explorer-1. Geophys. Res. Lett. 9, 933-936 (1982)

C.R. Chappell, T.E. Moore, J.H. Waite Jr., The Ionosphere as a fully adequate source of plasma for the earth's magnetosphere. J. Geophys. Res. 92, 5896-5910 (1987)

C.C. Chaston, J.W. Bonnell, C.W. Carlson, J.P. McFadden, R.E. Ergun, R.J. Strangeway, E.J. Lund, Auroral ion acceleration in dispersive Alfvén waves. J. Geophys. Res. 109, A04205 (2004). doi:10.1029/ 2003JA010053

C.C. Chaston, C.W. Carlson, J.P. McFadden, R.E. Ergun, R.J. Strangeway, How important are dispersive Alfvén waves for auroral particle acceleration? Geophys. Res. Lett. 34, L07101 (2007). doi:10.1029/ 2006GL029144

L.-J. Chen, C.A. Kletzing, S. Hu, S.R. Bounds, Auroral electron dispersion below inverted-V energies: resonant deceleration and acceleration by Alfvén waves. J. Geophys. Res. 110, A10S13 (2005). doi:10.1029/2005JA011168

J.H. Clemmons, J.H. Hecht, D.R. Salem, D.J. Strickland, Thermospheric density in the Earth's magnetic cusp as observed by the Streak mission. Geophys. Res. Lett. 35, L24103 (2008). doi:10.1029/2008GL035972

S. Close, M. Oppenheim, S. Hunt, L. Dyrud, Scattering characteristics of high-resolution meteor head echoes detected at multiple frequencies. J. Geophys. Res. 107(A10), 1295 (2002). doi:10.1029/2002JA009253 
M.V. Codrescu, T.J. Fuller-Rowell, On the importance of E-field variability for Joule heating in the highlatitude thermosphere. Geophys. Res. Lett. 22, 2393-2396 (1995)

W.R. Coley, R.A. Heelis, Adaptive identification and characterization of polar ionization patches. J. Geophys. Res. 100(A12), 23819-23827 (1995)

S.W.H. Cowley, Magnetosphere-Ionosphere Interactions: a tutorial review, in Magnetosphere Current Systems, ed. by S. Ohtani et al. AGU Geophys. Monograph, vol. 118 (2000)

P.D. Craven, D.L. Gallagher, R.H. Comfort, Relative concentration of $\mathrm{He}^{+}$in the inner magnetosphere as observed by the DE 1 retarding ion mass spectrometer. J. Geophys. Res. 102, 2279-2289 (1997)

G. Crowley, Dynamics of the Earth's thermosphere: a review. Rev. Geophys. 29, 1143-1165 (1991)

G. Crowley, R.R. Meier, Disturbed $\mathrm{O} / \mathrm{N}_{2}$ ratios and their transport to middle and low latitudes, in Midlatitude Ionospheric Dynamics and Disturbances, ed. by P.M. Kintner et al. AGU Geophys. Monograph, vol. 181 (2008). doi:10.1029/181GM06

W.D. Cumming, A.J. Dessler, Field-aligned currents in the magnetosphere. J. Geophys. Res. 72, 1007 (1967)

I. Daglis, R. Thorne, W. Baumjohann, S. Orsini, The terrestrial ring current: origin, formation, and decay. Rev. Geophys. 37, 407 (1999)

G.T. Davidson, Pitch angle diffusion and the origin of temporal and spatial structures in pulsating aurorae. Space Sci. Rev. 53, 45-82 (1990)

T.N. Davis, Observed characteristics of auroral forms. Space Sci. Rev. 22, 77 (1978)

O. de La Beaujardière, C/NOFS: a mission to forecast scintillations. J. Atmos. Sol.-Terr. Phys. 66, 1573-1591 (2004). doi:10.1016/j.jastp.2004.07.030

R. Doe, M. Mendillo, J. Vickrey, L. Zanetti, R. Eastes, Observations of nightside auroral cavities. J. Geophys. Res. 98, 293-310 (1993). doi:10.1029/92JA02004

R. Doe, J.F. Vickrey, M. Mendillo, Electrodynamic model for the formation of auroral ionospheric cavities. J. Geophys. Res. 100, 9683-9696 (1995)

J.W. Dungey, Interplanetary field and the auroral zones. Phys. Rev. Lett. 6, 47 (1961)

L.P. Dyrud, M.M. Oppenheim, A.F. vom Endt, The anomalous diffusion of meteor trails. Geophys. Res. Lett. 28(14), 2775-2778 (2001)

G.D. Earle, R.L. Bishop, Q. Zhou, S.P. Wallace, A comparative study of in-situ and remote intermediate layer measurements against wind model predictions of vertical ion drift. J. Atmos. Sol.-Terr. Phys. 60, 1313 (1998)

G.D. Earle, T.J. Kane, R.F. Pfaff, S.R. Bounds, Ion layer separation and equilibrium zonal winds in midlatitude sporadic E. Geophys. Res. Lett. 27, 461-464 (2000)

G.D. Earle, A.M. Musumba, S.L. Vadas, Satellite-based measurements of gravity wave-induced midlatitude plasma density perturbations. J. Geophys. Res. 113, A03303 (2008). doi:10.10299/5117JA012766

J.V. Eccles, A simple model of low-latitude electric fields. J. Geophys. Res. 103, 26699 (1998)

R.C. Elphic, J.W. Bonnell, R.J. Strangeway, L. Kepko, R.E. Ergun, J.P. McFadden, C.W. Carlson, W. Peria, C.A. Cattell, D. Klumpar, E. Shelley, W. Peterson, E. Moebius, L. Kistler, R. Pfaff, The auroral current circuit and field-aligned currents observed by FAST. Geophys. Res. Lett. 25, 2033-2036 (1998)

B.A. Emery, C. Lathuillere, P.G. Richards, R.G. Roble, M.J. Buonsanto, D.J. Knipp, P. Wilkinson, D.P. Siplerd, R. Niciejewski, Time dependent thermospheric neutral response to the 2-11 November 1993 storm period. J. Atmos. Sol.-Terr. Phys. 61, 329-350 (1999)

S.L. England, A review of the effects of non-migrating atmospheric tides on the earth's low-latitude ionosphere. Space Sci. Rev. (2011). doi:10.1007/s11214-011-9842-4

S.L. England, S. Maus, T.J. Immel, S.B. Mende, Longitudinal variation of the $E$-region electric fields caused by atmospheric tides. Geophys. Res. Lett. 33 (2006). doi:10.1029/2006GL027465

R.E. Ergun, L. Andersson, D. Main, Y.-J. Su, D.L. Newman, M.V. Goldman, C.W. Carlson, A.J. Hull, J.P. McFadden, F.S. Moser, Auroral particle acceleration by strong double layers: the upward current region. J. Geophys. Res. 109, A12220 (2004). doi:10.1029/2004JA010545

D.S. Evans, The observations of a near monoenergetic flux of auroral electrons. J. Geophys. Res. 73, 2315 (1968)

D.S. Evans, N.C. Maynard, J. Trøim, T. Jacobsen, A. Egeland, Auroral vector electric field and particle comparisons. 2. Electrodynamics of an arc. J. Geophys. Res. 82, 2235-2259 (1977)

D.T. Farley Jr., Two-stream plasma instability as a source of irregularities in the ionosphere. Phys. Rev. Lett. 10, 279-282 (1963)

D.T. Farley, E. Bonelli, B.G. Fejer, M.F. Larsen, The prereversal enhancement of the zonal electric field in the equatorial ionosphere. J. Geophys. Res. 91, 13723 (1986)

W.M. Farrell, T.L. Aggson, E.B. Rodgers, W.B. Hanson, Observations of ionospheric electric fields above atmospheric weather systems. J. Geophys. Res. 99, 19475-19483 (1994)

B.G. Fejer, D.T. Farley, B.B. Balsley, R.F. Woodman, Vertical structure of the VHF backscattering region in the equatorial electrojet and the gradient drift instability. J. Geophys. Res. 80(10), 1313-1324 (1975) 
B.G. Fejer, M.F. Larsen, D.T. Farley, Equatorial disturbance dynamo electric fields. Geophys. Res. Lett. 10, 537-540 (1983)

B.G. Fejer, J.W. Jensen, S.-Y. Su, Quiet time equatorial $F$ region vertical plasma drift model derived from ROCSAT-1 observations. J. Geophys. Res. 113, A05304 (2008). doi:10.1029/2007JA012801

J.M. Forbes, S.E. Palo, X. Zhang, Variability of the ionosphere. J. Atmos. Sol.-Terr. Phys. 62, 685-693 (2000)

J.C. Foster, W.J. Burke, SAPS: a new categorization for sub-auroral electric fields. Eos 83(3), 393-394 (2002). doi:10.1029/2002EO000289

J.C. Foster, H.B. Vo, Average characteristics and activity dependence of the subauroral polarization stream. J. Geophys. Res. 107(A12), 1475 (2002). doi:10.1029/2002JA009409

J.C. Foster, A.J. Coster, P.J. Erickson, J.M. Holt, F.D. Lind, W. Rideout, M. McCready, A. Van Eyken, R.J. Barnes, R.A. Greenwald, F.J. Rich, Multiradar observations of the polar tongue of ionization. J. Geophys. Res. 110, A09S31 (2005). doi:10.1029/2004JA010928

L.A. Frank, K.L. Ackerson, Observations of charged particle precipitation into the auroral zone. J. Geophys. Res. 76, 3612 (1971)

L.A. Frank, J.D. Craven, Imaging results from Dynamics Explorer 1. Rev. Geophys. 26(2), 249-283 (1988)

H.U. Frey, G. Haerendel, D. Knudsen, S. Buchert, O.H. Bauer, Optical and radar observations of the motion of auroral arcs. J. Atmos. Sol.-Terr. Phys. 58, 57-69 (1996)

T.J. Fuller-Rowell, D. Rees, S. Quegan, R.J. Moffett, M.V. Codrescu, G.H. Millward, A coupled thermosphere ionosphere model (CTIM), in Handbook of Ionospheric Models, STEP report, ed. by R.W. Schunk (1996), pp. 217-238

T.J. Fuller-Rowell, A.D. Richmond, N. Maruyama, Global modeling of storm-time thermospheric dynamics and electrodynamics, in Midlatitude Ionospheric Dynamics and Disturbances, ed. by P.M. Kintner et al. AGU Geophys. Monograph, vol. 181 (2008). doi:10.1029/181GM06

T.J. Fuller-Rowell, F. Wu, R. Akmaev, T.W. Fang, E. Araujo-Pradere, A whole atmosphere model simulation of the impact of a sudden stratospheric warming on thermosphere dynamics and electrodynamics. J. Geophys. Res. 115, A00G08 (2010). doi:10.1029/2010JA015524

Y.L. Galperin, Y.N. Ponomarov, A.G. Zosinova, Direct measurement of ion drift velocity in the upper atmosphere during a magnetic storm. Kosm. Issled. 11, 273 (1973)

S. Ganguli, The polar wind. Rev. Geophys. 34(3), 311-348 (1996). doi:10.1029/96RG00497

J.B. Gary, R.A. Heelis, W.B. Hanson, J.A. Slavin, Field-aligned Poynting flux observations in the highlatitude ionosphere. J. Geophys. Res. 99, 11417-11427 (1994)

W.D. Gonzalez, J.A. Joselyn, Y. Kamide, H.W. Kroehl, G. Rostoker, B.T. Tsurutani, V.M. Vasyliunas, What is a geomagnetic storm? J. Geophys. Res. 99, 5771-5794 (1994)

J.M. Grebowsky, A.C. Aiken, In situ measurements of meteoric ions, in Meteors in the Earth's Atmosphere, ed. by E. Murad, I.P. Williams (Cambridge University Press, Cambridge, 2002)

M.E. Greenspan, C.E. Rasmussen, W.J. Burke, M.A. Abdu, Equatorial density depletions observed at $840 \mathrm{~km}$ during the great magnetic storm of March 1989. J. Geophys. Res. 96, 13931-13942 (1991)

D.A. Gurnett, R.L. Huff, J.D. Menietti, J.L. Burch, J.D. Winningham, Correlated low-frequency electric and magnetic noise along the auroral field lines. J. Geophys. Res. 89, 8971-8985 (1984)

G. Haerendel, J.V. Eccles, The role of the equatorial electrojet in the evening ionosphere. J. Geophys. Res. 97, 1181 (1992)

G. Haerendel, S. Buchert, C. La Hoz, B. Raff, E. Rieger, On the proper motion of auroral arcs. J. Geophys. Res. 98, 6087-6099 (1993)

G. Haerendel, B.U. Olipitz, S. Buchert, O.H. Bauer, E. Rieger, C. La Hoz, Optical and radar observations of auroral arcs with emphasis on small scale structures. J. Atmos. Sol.-Terr. Phys. 58, 71-83 (1996)

C. Haldoupis, Midlatitude sporadic E: A typical paradigm of atmosphere-ionosphere coupling. Space Sci. Rev. (2011). doi:10.1007/11214-011-9786-8

T. Hallinan, Spiral-like auroral forms: observations and a proposed theory, Ph.D. thesis presented to the University of Alaska (1976)

T. Hallinan, T. Davis, Small-scale auroral arc distortions. Planet. Space Sci. 18, 1735 (1970)

J.K. Hargreaves, The Solar-Terrestrial Environment (Cambridge University Press, Cambridge, 1992)

R.A. Heelis, The effects of interplanetary magnetic field orientation on dayside high-latitude ionospheric convection. J. Geophys. Res. 89, 2873-2880 (1984)

R.A. Heelis, Studies of ionospheric plasma and electrodynamics and their application to ionospheremagnetosphere coupling. Rev. Geophys. 26, 317-328 (1988)

R.A. Heelis, Electrodynamics in the low and middle latitude ionosphere: a tutorial. J. Atmos. Sol.-Terr. Phys. 66, 825-838 (2004)

R.A. Heelis, S. Mohapatra, Storm time signatures of the ionospheric zonal ion drift at middle latitudes. J. Geophys. Res. 114, A02305 (2009). doi:10.1029/2008JA013620

R.A. Heelis, P.C. Kendall, R.J. Moffett, D.W. Windle, H. Rishbeth, Electrical coupling of the $E$-and $F$ regions and its effect on $F$-region drifts and winds. Planet. Space Sci. 22, 743 (1974) 
R.A. Heelis, P.H. Reiff, J.D. Winningham, W.B. Hanson, Ionospheric convection signatures observed by DE 2 during north-ward interplanetary magnetic field. J. Geophys. Res. 91, 5817-5830 (1986)

R.A. Heelis, Low- and middle-latitude ionospheric dynamics associated with magnetic storms, in Midlatitude Ionospheric Dynamics and Disturbances, ed. by P.M. Kintner et al. AGU Geophys. Monograph, vol. 181 (2008). doi:10.1029/181GM06

R.A. Heelis, W.R. Coley, A.G. Burrell, M.R. Hairston, G.D. Earle, M.D. Perdue, R.A. Power, L.L. Harmon, B.J. Holt, C.R. Loppincott, Behaviour of the $\mathrm{O}^{+} / \mathrm{H}^{+}$transition height during the extreme solar minimum of 2008. Geophys. Res. Lett. 36, L00C03 (2009a). doi:10.1029/2009GL038652

R.A. Heelis, J.J. Sojka, M. David, R.W. Schunk, Storm time density enhancements in the middle-latitude dayside ionosphere. J. Geophys. Res. 114, A03315 (2009b). doi:10.1029/2008JA013690

J.P. Heppner, Electric field variations durings aubstorms: OGO-6 measurements. Planet. Space Sci. 20, 14751498 (1972)

J.P. Heppner, N.C. Maynard, Empirical high-latitude electric field models. J. Geophys. Res. 92, 4467-4489 (1987)

J.P. Heppner, M.C. Liebrecht, N.C. Maynard, R.F. Pfaff, High-latitude distributions of plasma waves and spatial irregularities from DE 2 alternating current electric field observations. J. Geophys. Res. 98, 16291652 (1993)

C.O. Hines, The formation of midlatitude sporadic E layers. J. Geophys. Res. 69, 1018-1019 (1964)

C.-S. Huang, J.C. Foster, M.C. Kelley, Long-duration penetration of the interplanetary electric field to the lowlatitude ionosphere during the main phase of magnetic storms. J. Geophys. Res. 110, A11309 (2005). doi:10.1029/2005JA011202

J.D. Huba, G. Joyce, J.A. Fedder, Sami2 is another model of the ionosphere (SAMI2): a new low-latitude ionosphere model. J. Geophys. Res. 105, 23035-23053 (2000)

D.L. Hysell, E. Kudeki, Collisional shear instability in the equatorial F region ionosphere. J. Geophys. Res. 109, A11301 (2004)

D. Hysell, M. Larsen, Q. Zhou, Common volume coherent and incoherent scatter radar observations of midlatitude sporadic E-layer and QP echoes. Ann. Geophys. 22, 3277-3290 (2004)

D.L. Hysell, M.F. Larsen, C.M. Swenson, A. Barjatya, T.F. Wheeler, T.W. Bullett, M.F. Sarango, R.F. Woodman, J.L. Chau, D. Sponseller, Rocket and radar investigation of background electrodynamics and bottom-type scattering layers at the onset of equatorial spread F. Ann. Geophys. 24, 1387-1400 (2006)

T. Iijima, T.A. Potemra, Field-aligned currents in the dayside cusp observed by Triad. J. Geophys. Res. 34, 5971 (1976)

T.J. Immel, E. Sagawa, S.L. England, S.B. Henderson, M.E. Hagan, S.B. Mende, H.U. Frey, C.M. Swenson, L.J. Paxton, Control of equatorial morphology by atmospheric tides. Geophys. Res. Lett. 33, L15108 (2006). doi:10.1029/2006GL026161

D. Janches, L.P. Dyrud, S.L. Broadley, J.M.C. Plane, First observation of micrometeoroid differential ablation in the atmosphere. Geophys. Res. Lett. 36, L06101 (2009). doi:10.1029/2009GL037389

A.D. Johnstone, The mechanism of pulsating aurora. Ann. Geophys. 1, 397-410 (1983)

A.D. Johnstone, D.M. Walton, R. Liu, D.A. Hardy, Pitch angle diffusion of low-energy electrons by whistler mode waves. J. Geophys. Res. 98, 5959-5967 (1993)

B. Jones, P.J.S. Williams, K. Schlegel, T. Robinson, I. Häggström, Interpretation of enhanced electron temperatures measured in the auroral $E$-region during the ERRRIS campaign. Ann. Geophys. 9, 55-59 (1991)

G.O.L. Jones, P.J.S. Williams, K.J. Winser, Large ionospheric plasma flows along magnetic field lines: EISCAT observations and their interpretation. Adv. Space Res. 12, 157-160 (1992)

T. Karlsson, G. Marklund, L. Blomberg, A. Malkki, Subauroral electric fields observed by the Freja satellite: a statistical study. J. Geophys. Res. 103(A), 4327-4341 (1998). doi:10.1029/97JA00333

M.C. Kelley, The Earth's Ionosphere: Plasma Physics and Electrodynamics, 2nd edn. Int. Geophys. Ser, vol. 96 (Academic Press, Boston, 2009)

M.C. Kelley, C.W. Carlson, Observations of intense velocity shear and associated electrostatic waves near an auroral arc. J. Geophys. Res. 82, 2343-2348 (1977)

M.C. Kelley, C.L. Siefring, R.F. Pfaff, P.M. Kintner, M. Larsen, R. Green, R.H. Holzworth, L.C. Hale, J.D. Mitchell, D. LeVine, Electrical measurements in the atmosphere and the ionosphere over an active thunderstorm. 1. Campaign overview and initial ionospheric results. J. Geophys. Res. 90, 9815-9823 (1985). doi:10.1029/JA090iA10p09815

M.C. Kelley, J. LaBelle, E. Kudeki, B.G. Fejer, Sa. Basu, Su. Basu, K.D. Baker, C. Hanuise, P. Argo, R.F. Woodman, W.E. Swartz, D.T. Farley, J.W. Meriwether Jr., The Condor equatorial spread $F$ campaign: overview and results of the large-scale measurements. J. Geophys. Res. 91, 5487-5503 (1986). doi:10.1029/JA09liA05p05487

M.C. Kelley, D.J. Knudsen, J.F. Vickrey, Poynting flux measurements on a satellite: a diagnostic tool for space research. J. Geophys. Res. 96(A1), 201-207 (1991) 
M.C. Kelley, F. Garcia, J. Makela, T. Fan, E. Mak, C. Sia, D. Alcocer, Highly structured tropical airglow and TEC signatures during strong geomagnetic activity. Geophys. Res. Lett. 27, 465-468 (2000)

M.C. Kelley, J.J. Makela, A. Saito, The mid-latitude $F$ region at the mesoscale: some progress at last. J. Atmos. Sol.-Terr. Phys. 64, 1525-1529 (2002)

M.C. Kelley, J.J. Makela, O. de La Beaujardière, J. Retterer, Convective ionospheric storms: a review. Rev. Geophys. 49, RG2011 (2011). doi:10.1029/2010RG000340

T.L. Killeen, R.G. Roble, Thermospheric dynamics: contributions from the first 5 years of the Dynamics Explorer Program. Rev. Geophys. 26, 329-367 (1988)

T.L. Killeen, P.B. Hays, G.R. Carignan, R.A. Heelis, W.B. Hanson, N.W. Spencer, L.H. Brace, Ion-neutral coupling in the high-latitude $F$ region: evaluation of ion heating terms from Dynamics Explorer 2. J. Geophys. Res. 89, 7495-7508 (1984). doi:10.1029/JA089iA09p07495

P.M. Kintner Jr., A.J. Coster, T. Fuller-Rowell, A.J. Mannucci, M. Mendillo, R. Heelis (eds), Midlatitude Ionospheric Dynamics and Disturbances, AGU Geophys. Monograph, vol. 181 (2008). doi:10.1029/ $181 \mathrm{GM} 06$

S. Kirkwood, H. Opgenoorth, J.S. Murphree, Ionospheric conductivities, electric fields and currents associated with auroral substorms measured by the EISCAT radar. Planet. Space Sci. 36(12), 1359-1380 (1988)

J.H. Klenzing, D.E. Rowland, R.F. Pfaff, G. Le, H. Freudenreich, R.A. Haaser, A.G. Burrell, R.A. Stoneback, W.R. Coley, R.A. Heelis, Observations of low-latitude plasma density enhancements and their associated plasma drifts. J. Geophys. Res. 116, A09324 (2011). doi:10.1029/2011JA016711

S. Knight, Parallel electric fields. Planet. Space Sci. 21, 741 (1973)

D.J. Knudsen, M.C. Kelley, G.D. Earle, J.F. Vickrey, M. Boehm, Distinguishing Alfvén waves from quasistatic field structures associated with the discrete aurora: sounding rocket and HILAT satellite measurements. Geophys. Res. Lett. 17(7), 921-924 (1990)

E. Kopp, On the abundance of metal ions in the lower ionosphere. J. Geophys. Res. 102(A5), 9667-9674 (1997)

E. Kudeki, S. Bhattacharyya, Postsunset vortex in equatorial $F$-region plasma drifts and implications for bottomside spread-F. J. Geophys. Res. 104(A), 28163-28170 (1999). doi:10.1029/1998JA900111

E. Kudeki, D.T. Farley, B.G. Fejer, Long wavelength irregularities in the equatorial electrojet. Geophys. Res. Lett. 9, 684 (1982)

E. Kudeki, B.G. Fejer, D.T. Farley, C. Hanuise, The Condor equatorial electrojet campaign: radar results. J. Geophys. Res. 92, 13,561-13,577 (1987)

E. Kudeki, A. Akgiray, M. Milla, J.L. Chau, D.L. Hysell, Equatorial spread-F initiation: post-sunset vortex, thermospheric winds, gravity waves. J. Atmos. Sol.-Terr. Phys. 69, 2416-2427 (2007)

J.W. LaBelle III, Ionospheric Turbulence: Case Studies in Equatorial Spread F and Development of a RocketBorne Interferometer, Ph.D. thesis presented to Cornell University (1985)

M.F. Larsen, A shear instability seeding mechanism for quasi-periodic radar echoes. J. Geophys. Res. 105, 24931-24940 (2000)

M.F. Larsen, S. Fukao, M. Yamamoto, R. Tsunoda, K. Igarashi, T. Ono, The SEEK chemical release experiment: observed neutral wind profile in a region of sporadic-E. Geophys. Res. Lett. 25, 1789-1792 (1998)

M.F. Larsen, M. Yamamoto, S. Fukao, R. Tsunoda, A. Saito, Observations of neutral winds, winds shears, and wave structure during a sporadic-E/QP event. Ann. Geophys. 23, 2369-2375 (2005)

G. Le, G. Lu, R.J. Strangeway, R.F. Pfaff Jr., Strong interplanetary magnetic field $\mathrm{B}_{\mathrm{y}}$-related plasma convection in the ionosphere and cusp field-aligned currents under northward interplanetary magnetic field conditions. J. Geophys. Res. 107, 1477 (2002). doi:10.1029/2001JA007546

G. Le, C.-S. Huang, R.F. Pfaff, S.-Y. Su, H.-C. Yeh, R.A. Heelis, F.J. Rich, M. Hairston, Plasma density enhancements associated with equatorial spread F: ROCSAT-1 and DMSP observations. J. Geophys. Res. 108, 1318 (2003). doi:10.1029/2002JA009592

G. Le, J.A. Slavin, R.J. Strangeway, Space Technology 5 observations of the imbalance of regions 1 and 2 field-aligned currents and its implication to the cross-polar cap Pedersen currents. J. Geophys. Res. 115, A07202 (2010). doi:10.1029/2009JA014979

C.H. Lin, C.C. Hsiao, J.Y. Liu, C.H. Liu, Longitudinal structure of the equatorial ionosphere: time evolution of the four-peaked EIA structure. J. Geophys. Res. 112, A12305 (2007). doi:10.1029/2007JA012455

G. Lu, A. Richmond, B. Emery, R. Roble, Magnetosphere-ionosphere-thermosphere coupling: effect of neutral winds on energy transfer and field-aligned current. J. Geophys. Res. 100, 19643-19659 (1995). doi:10.1029/95JA00766

G. Lu, L.P. Goncharenko, A.D. Richmond, R.G. Roble, N. Aponte, A dayside ionospheric positive storm phase driven by neutral winds. J. Geophys. Res. 113, A08304 (2009). doi:10.1029/2007JA012895

A.T. Lui, Y.P. Perrault, S.-I. Akasofu, C.D. Anger, The diffuse aurora. Planet. Space Sci. 21,857 (1973)

D. Lummerzheim, Modeling and forecasting aurora. Computing in Sci. Eng. 9(5), 53-61 (2007) 
L.R. Lyons, Formation of auroral arcs via magnetosphere-ionosphere coupling. Rev. Geophys. 30, 93-112 (1992)

R. Lysak, Generalized model of the ionospheric Alfvén resonator, in Auroral Plasma Dynamics, ed. by R. Lysak. AGU Geophys. Monograph, vol. 80 (1993)

J.J. Makela, Y. Otsuka, Overview of nighttime ionospheric instabilities at low- and mid-latitudes: coupling aspects resulting in structuring at the mesoscale. Space Sci. Rev. (2011). doi:10.1007/s11214-011-9816-6

J.J. Makela, S.A. Gonzalez, B. MacPherson, X. Pi, M.C. Kelley, P.J. Sultan, Intercomparisons of total electron content measurements using the Arecibo incoherent scatter radar and GPS. Geophys. Res. Lett. 27(18), 2841-2844 (2000)

A.J. Mallinckrodt, C.W. Carlson, On the anticorrelation of the electric field and peak electron energy within an auroral arc. J. Geophys. Res. 90, 399-408 (1985)

A.J. Mannucci, B.T. Tsurutani, B.A. Iijima, A. Komjathy, A. Saito, W.D. Gonzalez, F.L. Guarnieri, J.U. Kozyra, R. Skoug, Dayside global ionospheric response to the major interplanetary events of October 29-30, 2003 "Halloween Storms". Geophys. Res. Lett. 32, L12S02 (2005). doi:10.1029/ 2004GL021467

G.T. Marklund, Auroral arc classification scheme based on the observed arc-associated electric field pattern. Planet. Space Sci. 32, 193-211 (1984)

G.T. Marklund, Electric fields and plasma processes in the auroral downward current region, below, within, and above the acceleration region. Space Sci. Rev. 142, 1-21 (2009). doi:10.1007/s11214-008-9373-9

G.T. Marklund, L. Blomberg, C.-G. Falthämmar, P.-A. Lindqvist, On intense diverging electric fields associated with black aurora. Geophys. Res. Lett. 21, 1859-1862 (1994)

G. Marklund, T. Karlsson, J. Clemmons, On low-altitude particle acceleration and intense electric fields and their relationship to black aurora. J. Geophys. Res. 102, 17509-17522 (1997). doi:10.1029/97JA00334

D.F. Martyn, Atmospheric tides in the ionosphere. I. Solar tides in the F2 region. Proc. R. Soc. Lond. Ser. A, Math. Phys. Sci. 189, 241-260 (1947)

J.D. Mathews, Sporadic E: current views and recent progress. J. Atmos. Sol.-Terr. Phys. 60, 413-435 (1998)

J.D. Matthews, S. Briczinski, D. Meisel, C. Heinselman, Radio and meteor science outcomes from comparisons of meteor radar observations at AMISR Poker Flat, Sondrestrom, and Arecibo. Earth Moon Planets 102, 365-372 (2008). doi:10.1007/s11038-007-9168-0

S. Matsushita, Global presentation of the external $S_{q}$ and $L$ current systems. J. Geophys. Res. 70, 4395 (1965)

N.C. Maynard, A. Bahnsen, P. Christophersen, A. Egeland, R. Lundin, An example of anticorrelation of auroral particles and electric fields. J. Geophys. Res. 78, 3976-3980 (1973)

J.P. McFadden, C.W. Carlson, R.E. Ergun, Microstructure of the auroral acceleration region as observed by FAST. J. Geophys. Res. 104, 14453-14480 (1999)

C. McIlwain, Direct measurements of particles producing visible aurora. J. Geophys. Res. 65, 2727 (1960)

R.L. McPherron, Magnetosphere dynamics, in Introduction to Space Physics, ed. by M.G. Kivelson, C.T. Russell (Cambridge University Press, Cambridge, 1995)

R.R. Meier, G. Crowley, D.J. Strickland, A.B. Christensen, L.J. Paxton, D. Morrison, C.L. Hackert, First look at the 20 November 2003 superstorm with TIMED/GUVI: comparisons with a thermospheric global circulation model. J. Geophys. Res. 110 (2005). doi:10.1029/2004JA010990

M. Mendillo, Storms in the ionosphere: patterns and processes for total electron content. Rev. Geophys. 44, RG4001 (2006). 1-47

H.R. Middleton, S.E. Pryse, L. Kersley, G.S. Bust, E.J. Fremouw, J.A. Secan, Evidence for the tongue of ionization under northward interplanetary magnetic field conditions. J. Geophys. Res. 110, A07301 (2005). doi:10.1029/2004JA010800

T. Nagai, J.H. Waite Jr., J.L. Green, C.R. Chappell, First measurements of supersonic polar wind in the polar magnetosphere. Geophys. Res. Lett. 11, 669-672 (1984)

P.T. Newell, C.-I. Meng, K.M. Lyons, Suppression of discrete aurorae by sunlight. Lett. Nat. 381, 766-767 (1996)

P.T. Newell, T. Sotirelis, S. Wing, Diffuse, monoenergetic, and broadband aurora: the global precipitation budget. J. Geophys. Res. 114, A09207 (2009). doi:10.1029/2009JA014326

Y. Nishimura, J. Bortnik, W. Li, R.M. Thorne, L.R. Lyons, V. Angelopoulos, S.B. Mende, J.W. Bonnell, O.L. Contel, C. Cully, R. Ergun, U. Auster, Identifying the driver of pulsating aurora. Science 330, 81-84 (2010). doi:10.1126/science.1193186

Y. Otsuka, K. Shiokawa, T. Ogawa, P. Wilkinson, Geomagnetic conjugate observations of medium-scale traveling ionospheric disturbances at mid-latitude using all-sky airglow imagers. Geophys. Res. Lett. 31, L15803 (2004). doi:10.1029/2004GL020262

G. Paschmann, S. Haaland, R. Treumann, Auroral Plasma Physics (Kluwer Academic, Dordrecht, 2003)

W.J. Peria, C.W. Carlson, R.E. Ergun, J.P. McFadden, J. Bonnell, R.C. Elphic, R.J. Strangeway, Characteristics of field-aligned currents near the auroral acceleration region: FAST observations, in Magnetosphere Current Systems, ed. by S. Ohtani et al. AGU Geophys. Monograph, vol. 118 (2000) 
R.F. Pfaff, Rocket Studies of Plasma Turbulence in the Equatorial and Auroral Electrojets. Ph.D. thesis presented to Cornell University (1986)

R.F. Pfaff Jr., In-situ measurement techniques for ionospheric research, in Modern Ionospheric Science, ed. by H. Kohl et al. (Max-Planck-Institut für Aeronomie, Katlenburg-Lindau, 1996)

R.F. Pfaff, M.C. Kelley, E. Kudeki, B.G. Fejer, K.D. Baker, Electric field and plasma density measurements in the strongly driven daytime equatorial electrojet. 1. The unstable layer and gradient drift waves. J. Geophys. Res. 92, 13,578-13,596 (1987)

R.F. Pfaff, J. Heppner, W. Hoegy, J. Slavin, N. Maynard, M.C. Liebrecht, A survey of enhanced sub-auroral electric fields observed by Dynamics Explorer-2, In: EOS Trans. Am. Geophys. Union, Fall Meeting (1988)

R.F. Pfaff, J. Sahr, J.F. Providakes, W.E. Swartz, D.T. Farley, P.M. Kintner, I. Haggstrom, A. Hedberg, H. Opgenoorth, G. Holmgren, The E-region Rocket/Radar Instability Study (ERRRIS)—scientific objectives and campaign overview. J. Atmos. Sol.-Terr. Phys. 54, 779-808 (1992)

R.F. Pfaff, M.H. Acuna, P.A. Marionni, N.B. Trivedi, DC polarization electric field, current density, and plasma density measurements in the daytime equatorial electrojet. Geophys. Res. Lett. 24, 1667-1670 (1997a)

R.F. Pfaff, P.A. Marionni, W.E. Swartz, Wavevector observations of the two-stream instability in the daytime equatorial electrojet. Geophys. Res. Lett. 24, 1671-1674 (1997b)

R.F. Pfaff, J. Clemmons, C. Carlson, R. Ergun, J. McFadden, F. Mozer, M. Temerin, D. Klumpar, W. Peterson, E. Shelley, E. Moebius, L. Kistler, R. Strangeway, R. Elphic, C. Cattell, Initial FAST observations of acceleration processes in the cusp. Geophys. Res. Lett. 25, 2037-2040 (1998)

R.F. Pfaff et al., Combined sounding rocket and SuperDarn/EISCAT radar observations of plasma convection, shear, irregularities, and other phenomena in the cusp and boundary layer during IMF $B_{z}$ negative and By negative conditions. In: EOS Trans. Am. Geophys. Union, Fall Meeting (2003)

R.F. Pfaff, C. Liebrecht, J.-J. Berthelier, M. Malingre, M. Parrot, J.-P. Lebreton, DEMETER satellite observations of plasma irregularities in the topside ionosphere at low, middle, and sub-auroral latitudes and their dependence on magnetic storms, in Midlatitude Ionospheric Dynamics and Disturbances, ed. by P.M. Kitner et al. AGU Geophys. Monograph, vol. 181 (2008). doi:10.1029/181GM06

J.M. Picone, A.E. Hedin, D.P. Drob, A.C. Aikin, NRLMSISE-00 empirical model of the atmosphere: statistical comparisons and scientific issues. J. Geophys. Res. 107(A12), 1468 (2002). doi:10.1029/2002JA009430

W.E. Potter, L.J. Cahill Jr., Electric and magnetic field measurements near an auroral electrojet. J. Geophys. Res. 74, 5159-5160 (1969)

E. Priest, T. Forbes, Magnetic Reconnection, MHD Theory and Applications (Cambridge University Press, Cambridge, 2000)

G.W. Prölss, Ionospheric F-region storms, in Handbook of Atmosp. Electrodynamics, 2, ed. by H. Volland (CRC Press, Boca Raton, 1995), Chap. 8, pp. 195-248

G.W. Prölss, Magnetic storm associated perturbations of the upper atmosphere, in Magnetic Storms, ed. by B. Tsurutani et al. AGU Geophys. Monograph Series, vol. 98 (1997)

G. Prölss, Physics of the Earth's Space Environment: an Introduction (Springer, Heidelberg, 2004)

M.H. Rees, Physics and Chemistry of the Upper Atmosphere. Cambridge Atmos. and Space Sci. Series (Univ. Press, Cambridge, 1989)

P. Reiff, T. Hill, J. Burch, Solar wind plasma injection at the dayside magnetospheric cusp. J. Geophys. Res. 82, 479 (1977)

A.D. Richmond, G. Lu, Upper-atmospheric effects of magnetic storms: a brief tutorial. J. Atmos. Sol.-Terr. Phys. 62, 1115-1127 (2000)

A.D. Richmond, J.P. Thayer, Ionospheric electrodynamics: a tutorial, in Magnetosphere Current Systems, ed. by S. Ohtani et al. AGU Geophys. Monograph, vol. 118 (2000)

A.D. Richmond, E. Ridley, R. Roble, A thermosphere/ionosphere general-circulation model with coupled electrodynamics. Geophys. Res. Lett. 19, 601-604 (1992). doi:10.1029/92GL00401

H. Rishbeth, The effect of winds on the ionospheric F2-peak. J. Atmos. Sol.-Terr. Phys. 29, 225-288 (1967)

H. Rishbeth, The F-layer dynamo. Planet. Space Sci. 19, 263-267 (1971)

H. Rishbeth, The ionospheric $E$-layer and $F$-layer dynamos-a tutorial review. J. Atmos. Sol.-Terr. Phys. 59, 1873-1880 (1997)

H. Rishbeth, O.K. Garriot, Introduction to Ionospheric Physics (Academic Press, New York, 1969)

H. Rishbeth, T.J. Fuller-Rowell, A.S. Rodger, F-layer storms and thermospheric composition. Phys. Scr. 36, 327-336 (1987)

R.G. Roble, E. Ridley, A. Richmond, R. Dickinson, A coupled thermosphere ionosphere general-circulation model. Geophys. Res. Lett. 15, 1325-1328 (1988). doi:10.1029/GL015I012P01325

A. Rodger, The mid-latitude trough-revisited, in Midlatitude Ionospheric Dynamics and Disturbances, ed. by P.M. Kintner et al. AGU Geophys. Monograph, vol. 181 (2008). doi:10.1029/181GM06 
A. Saito, M. Nishimura, M. Yamamoto, S. Fukao, M. Kubota, K. Shiokawa, Y. Otsuka, T. Tsugawa, T. Ogawa, M. Ishii, T. Sakanoi, S. Miyazaki, Traveling ionospheric disturbances detected in the FRONT campaign. Geophys. Res. Lett. 28, 689-692 (2001)

N. Sato, D.M. Wright, C.W. Carlson, Y. Ebihara, M. Sato, T. Saemundsson, S. Milan, M. Lester, Generation region of pulsating aurora obtained simultaneously by the FAST satellite and a Syowa-Iceland conjugate pair of observations. J. Geophys. Res. 109, A10201 (2004). doi:10.1029/2004JA010419

L. Scherliess, B.G. Fejer, Storm time dependence of equatorial disturbance dynamo zonal electric fields. J. Geophys. Res. 102(A11), 24,037-24,046 (1997)

L. Scherliess, B.G. Fejer, Radar and satellite global equatorial $F$ region vertical drift model. J. Geophys. Res. 104, 6829-6842 (1999). doi:10.1029/1999JA900025

K. Schlegel, J.P. St.-Maurice, Anomalous heating of the polar $E$ region by unstable plasma waves. 1. Observations. J. Geophys. Res. 86(A3), 1447-1452 (1981)

R. Schunk, P.M. Banks, Effects of electric fields and other processes upon the nighttime high-latitude $F$ layer. J. Geophys. Res. 81, 3271-3282 (1976)

R. Schunk, A.F. Nagy, Ionospheres: Physics, Plasma Physics, and Chemistry (Cambridge Univ. Press, Cambridge, 2009)

R.W. Schunk, J.J. Sojka, Global ionosphere-polar wind system during changing magnetic activity. J. Geophys. Res. 102, 11,625-11,651 (1997)

R.W. Schunk, J.J. Sojka, M.D. Bowline, Theoretical study of the electron temperature in the high-latitude ionosphere for solar maximum and winter conditions. J. Geophys. Res. 91, 12,041-12,054 (1986)

V.A. Sergeev, V. Angelopoulos, R. Nakamura, Recent advances in understanding substorm dynamics. Geophys. Res. Lett. 39(5), L05101 (2012) (ISSN 0094-8276). doi:10.1029/2012GL050859

E.G. Shelley, R.D. Sharp, R.G. Johnson, Satellite observations of an ionospheric acceleration mechanism. Geophys. Res. Lett. 3, 654 (1976)

K. Shiokawa, Y. Otsuka, T. Ogawa, P. Wilkinson, Time evolution of high-altitude plasma bubbles imaged at geomagnetic conjugate points. Ann. Geophys. 22, 3137-3143 (2004)

F. Simoes, R. Pfaff, J.-J. Berthelier, J. Klenzing, A review of low frequency electromagnetic wave phenomena related to tropospheric-ionospheric coupling mechanisms. Space Sci. Rev. (2011). doi:10.1007/s112140-011-9854-0

G.L. Siscoe, K.D. Siebert, Bimodal nature of solar wind-magnetosphere-ionosphere-thermosphere coupling. J. Atmos. Sol.-Terr. Phys. 68, 911-920 (2006)

M. Smiddy, M.C. Kelley, W. Burke, F. Rich, R. Sagalyn, B. Shuman, R. Hays, S. Lai, Intense poleward directed electric fields near the ionospheric projection of the plasmapause. Geophys. Res. Lett. 4, 543 (1977)

M. Smiddy, W.J. Burke, M.C. Kelley, N.A. Saflekos, M.S. Gussenhoven, D.A. Hardy, F.J. Rich, Effects of high-latitude conductivity on observed convection electric fields and Birkeland currents. J. Geophys. Res. 85, 6811-6818 (1980)

J.J. Sojka, W.J. Raitt, R.W. Schunk, Plasma density features associated with strong convection in the winter high-latitude $F$ region. J. Geophys. Res. 86, 6916-6968 (1981)

R.W. Spiro, R.A. Heelis, W.B. Hanson, Rapid subauroral ion drifts observed by Atmosphere Explorer C. Geophys. Res. Lett. 6, 657-660 (1979)

H. Stenbaek-Nielsen, T. Hallinan, D. Osborne, J. Kimball, C. Chaston, J. McFadden, G. Delory, M. Temerin, C. Carlson, Aircraft observations conjugate to FAST. Geophys. Res. Lett. 25, 2073 (1998)

R.J. Strangeway, The equivalence of Joule dissipation and frictional heating in the collisional ionosphere. J. Geophys. Res. 117, A02310 (2012). doi:10.1029/2011JA017302

R. Strangeway, C.T. Russell, C.W. Carlson, J.P. McFadden, R.E. Ergun, M. Temerin, D.M. Klumpar, W.K. Peterson, T.E. Moore, Cusp field-aligned currents and ion outflows. J. Geophys. Res. 105(A9), 21129_ $21142(2000)$

D.J. Strickland, J.R. Jasperse, J.A. Whalen, Dependence of auroral FUV emissions on the incident electron spectrum and neutral atmosphere. J. Geophys. Res. 88, 8051 (1983)

D.J. Strickland, R.E. Daniell Jr., J.R. Jasperse, B. Basu, Transport-theoretic model for the electron-protonhydrogen atom aurora. 2. Model results. J. Geophys. Res. 98, 21533-21548 (1993)

D.J. Strickland, R.E. Daniell, J.D. Craven, Negative ionospheric storm coincident with DE 1-observed thermospheric disturbance on October 14, 1981. J. Geophys. Res. 106(A10), 21049-21062 (2001)

Y.-J. Su, J.L. Horwitz, T.E. Moore, B.L. Giles, M.O. Chandler, P.D. Craven, M. Hirahara, C.J. Pollock, Polar wind survey with the Thermal Ion Dynamics Experiment/Plasma Source Instrument suite aboard POLAR. J. Geophys. Res. 103(A12), 29,305-29,337 (1998)

R.N. Sudan, J. Akinrimisi, D.T. Farley, Generation of small-scale irregularities in the equatorial electrojet. J. Geophys. Res. 78(1), 240 (1973)

M. Sugiura, N.C. Maynard, W.H. Farthing, J.P. Heppner, B.G. Ledley, L.J. Cahill Jr., Initial results on the correlation between the magnetic and electric fields observed from the DE-2 satellite in the field-aligned current regions. Geophys. Res. Lett. 9, 985-988 (1982) 
W.E. Swartz, M.C. Kelley, J.J. Makela, S.C. Collins, E. Kudeki, S. Franke, J. Urbina, N. Aponte, M.P. Sulzer, S.A. González, Coherent and incoherent scatter radar observations during intense mid-latitude spread $F$. Geophys. Res. Lett. 27(18), 2829-2832 (2000)

J.P. Thayer, Height-resolved Joule heating rates in the high-latitude $E$ region and the influence of neutral winds. J. Geophys. Res. 103, 471-487 (1998)

J.P. Thayer, High-latitude currents and their energy exchange with the ionosphere-thermosphere system. J. Geophys. Res. 105, 23,015-23,024 (2000)

J.P. Thayer, J. Semeter, The convergence of magnetospheric energy flux in the polar atmosphere. J. Atmos. Sol.-Terr. Phys. 66, 807-824 (2004)

J.P. Thayer, J.F. Vickrey, R.A. Heelis, J.B. Gary, Interpretation and modeling of the high-latitude electromagnetic energy flux. J. Geophys. Res. 100, 19,715-19,728 (1995)

R.M. Thorne, B. Ni, X. Tao, R.B. Horne, N.P. Meredith, Scattering by chorus waves as the dominant cause of diffuse auroral precipitation. Nature 467, 943-946 (2010)

R.T. Tsunoda, High-latitude $F$ region irregularities: a review and synthesis. Rev. Geophys. 26, 719-760 (1988). doi:10.1029/RG026i004p00719

R.T. Tsunoda, R.C. Livingston, J.P. McClure, W.B. Hanson, Equatorial plasma bubbles: vertically elongated wedges from the bottomside $F$ layer. J. Geophys. Res. 87, 9171-9180 (1982)

S.L. Vadas, D.C. Fritts, Thermospheric responses to gravity waves: Influences of increasing velocity and thermal diffusivity. J. Geophys. Res. 110, D15103 (2005). doi:10.1029/2004JD005574

S.L. Vadas, Horizontal and vertical propagation and dissipation of gravity waves in the thermosphere from lower atmosphere and thermospheric sources. J. Geophys. Res. 112, A06305 (2007). doi:10.1029/2006JA011845

C.E. Valladares, D.T. Decker, R. Sheehan, Modeling the formation of polar cap patches using large plasma flows. Radio Sci. 31, 573-593 (1996)

T. Vondrak, J.M.C. Plane, S. Broadley, D. Janches, A chemical model of meteoric ablation. Atmos. Chem. Phys. 8, 7015-7031 (2008)

J.-E. Wahlund, H.J. Opgenoorth, F.R.E. Forme, M.A.L. Persson, I. Häggström, J. Lilensten, Electron energization in the topside auroral ionosphere: on the importance of ion-acoustic turbulence. J. Atmos. Sol.-Terr. Phys. 55, 623-645 (1993)

R.L. Walterscheid, Gravity wave transports and their effects on the large-scale circulation of the upper mesosphere and lower thermosphere. Adv. Space Res. 27, 1713-1721 (2001)

D.R. Weimer, Models of high-latitude electric potentials derived with a least error fit of spherical harmonic coefficients. J. Geophys. Res. 100, 19,595-19,607 (1995). doi:10.1029/95JA01755

J.D. Whitehead, Production and prediction of sporadic E. Rev. Geophys. 8, 65-144 (1970)

P.J.S. Williams, B. Jones, G.O.L. Jones, The measured relationship between electric field strength and electron temperature in the auroral E-region. J. Atmos. Sol.-Terr. Phys. 54, 741-748 (1992)

C.T.R. Wilson, Investigation on lightning discharges and on the electric field of thunderstorms. Philos. Trans. R. Soc. Lond. A221, 73-115 (1920)

R.F. Woodman, Spread $F$ - an old equatorial aeronomy problem finally resolved? Ann. Geophys. 27, 19151934 (2009)

R.F. Woodman, C. La Hoz, Radar observations of $F$ region equatorial irregularities. J. Geophys. Res. 81, 5447-5466 (1976)

J. Wygant, D. Rowland, H. Singer, M. Temerin, F. Mozer, M. Hudson, Experimental evidence on the role of the large spatial-scale electric field in creating the ring current. J. Geophys. Res. 103, 29527 (1998)

J.R. Wygant, A. Keiling, C.A. Cattell, M. Johnson, R.L. Lysak, M. Temerin, F.S. Mozer, C.A. Kletzing, J.D. Scudder, W. Peterson, C.T. Russell, G. Parks, M. Brittnacher, G. Germany, J. Spann, Polar spacecraft based comparisons of intense electric fields and Poynting flux near and within the plasma sheet-tail lobe boundary to UVI images: an energy source for the aurora. J. Geophys. Res. 105, 18,675-18,692 (2000)

M. Yamamoto, S. Fukao, R.F. Woodman, T. Ogawa, T. Tsuda, S. Kato, Mid-latitude $E$ region field-aligned irregularities observed with the MU radar. J. Geophys. Res. 96, 15,943-15,949 (1991). doi:10.1029/ 91JA01321

M. Yamauchi, R. Lundin, L. Eliasson, S. Ohtani, J. Clemmons, Relationship between large-, meso-, and small-scale field aligned currents and their current carriers, in Polar Cap Boundary Phenomena, ed. by J. Moen et al. (1998), pp. 173-188

T. Yokoyama, D.L. Hysell, Y. Otsuka, M. Yamamoto, Three-dimensional simulation of the coupled Perkins and $E_{S}$-layer instabilities in the nighttime midlatitude ionosphere. J. Geophys. Res. 114, A03308 (2009). doi:10.1029/2008JA013789

L.J. Zanetti, W. Baumjohann, T.A. Potemra, P.F. Bythrow, Three-dimensional Birkeland-ionospheric current system, determined from MAGSAT, in Magnetospheric Currents, ed. by T.A. Potemra. AGU Monograph, vol. 28 (1984)

A.J. Zmuda, Ionization enhancement from Van Allen electrons in South Atlantic magnetic anomaly. J. Geophys. Res. 71, 1911 (1966) 